Marcelo Araújo da Silva

\title{
aplicação do lagrangeano aumentado em otimização estrutural com restrições dinâmicas
}

Dissertação Apresentada à Escola Politécnica da Universidade de São Paulo para a Obtenção do Título de Mestre em Engenharia.

São Paulo

1997

Marcelo Araújo da Silva 


\section{aplicação do lagrangeano aumentado em otimização estrutural com restrições dinâmicas}

Dissertação Apresentada à Escola Politécnica da Universidade de São Paulo para a Obtenção do Título de Mestre em Engenharia.

Área de Concentração:

Engenharia de Estruturas

Orientadora:

Priscila Goldenberg

São Paulo

1997 
À minha avó Adelina e aos meus pais, Onofre e Marielva. 
Gostaria de agradecer

À minha orientadora Prof ${ }^{a}$. Dr. ${ }^{a}$ Priscila Goldenberg pela orientação, incansável atenção e estímulo dispensados durante a realização deste trabalho.

Aos Prof. Dr. Reyolando M. L. R. F. Brasil e Prof. Dr. Paulo de Mattos Pimenta pelo grande apoio, e auxilio nas aplicações desenvolvidas.

À minha esposa Gislene e à irmã Ticiana pelo grande incentivo e satisfação em verem este trabalho concluído.

Aos amigos Petrônio e Adriano pelo apoio e amizade.

À todos os professores e funcionários do Departamento de Engenharia de Estruturas e Fundações que direta ou indiretamente contribuíram para a realização deste trabalho.

Ao Conselho Nacional de Desenvolvimento Científico e Tecnológico - CNPq - pelo apoio financeiro. 


\section{SUMÁRIO}

LISTA DE FIGURAS

LISTA DE TABELAS

LISTA DE GRÁFICOS

RESUMO

“ABSTRACT"

1 INTRODUÇÃO

2 CONCEITOS MATEMÁTICOS BÁSICOS

$\begin{array}{lll}2.1 & \text { Espaços Vetoriais } & 04\end{array}$

2.2 Espaços Vetoriais Euclidianos e Espaços Normados

06

2.3 Funções de Várias Variáveis Reais 08

$\begin{array}{lll}2.4 & \text { Seqüências } & 15\end{array}$

$\begin{array}{lll}2.5 & \text { Funcionais } & 16\end{array}$

$\begin{array}{ll}2.6 & \text { Equações Diferenciais Ordinárias } \\ \end{array}$

$\begin{array}{lll}2.7 & \text { Programação Matemática } & 21\end{array}$

2.7.1 Otimização sem Restrições $\quad 21$

2.7.2 Otimização com Restrições 22

3 PROBLEMA DE OTIMIZAÇÃO COM RESTRIÇÕES DINÂMICAS 25

$\begin{array}{lll}3.1 & \text { Introdução } & 25\end{array}$

3.2 Formulação do Problema 26 
3.3 Método do Lagrangeano Aumentado

27

3.3.1 Introdução

3.3.2 Lagrangeano Aumentado

3.3.3 Condições Necessárias e Suficientes 31

3.3.4 Propriedades de Convergência 33

3.3.5 O Algoritmo do Método dos Multiplicadores 34

3.3.6 O Método do Lagrangeano Aumentado em Problemas com Restrições Dinâmicas

4 MÉTODOS NUMÉRICOS

4.1 Solução de Sistemas Lineares

41

4.2 Métodos de Integração Numérica $\quad 42$

4.2.1 Introdução $\quad 42$

4.2.2 Fórmulas de Integração Newton-Cotes 43

$\begin{array}{lll}\text { 4.2.3 Quadratura de Gauss } & 45\end{array}$

$\begin{array}{lll}4.3 & \text { Interpolação Polinomial } & 48\end{array}$

$\begin{array}{lll}4.3 .1 & \text { Introdução } & 48\end{array}$

4.3.2 Interpolação com Funções Splines Cúbicos 49

4.4 Métodos de Solução de Sistemas de Equações Diferenciais Ordinárias de Primeira e Segunda Ordem 52

4.4.1 Introdução $\quad 52$

4.4.2 Métodos Runge-Kutta de Quarta e Quinta Ordem 52

4.4.3 Método de Newmark $\quad 54$

4.5 Algoritmo de Minimização sem Restrições 56 
4.6 Método da Diferenças Finitas

58

5 EXEMPLOS RESOLVIDOS PELO MÉTODO DOS

MULTIPLICADORES

5.1 Exemplo 1 - Pórtico Elástico Linear com um Grau de Liberdade 61

5.2 Exemplo 2 - Pórtico Viscoelastoplástico com um Grau de Liberdade 67

5.3 Exemplo 3 - Um Absorvedor de Impacto Linear 72

5.4 Exemplo 4 - Um Absorvedor de Impacto Não-linear 78

5.5 Exemplo 5 - Otimização de um Isolador de Vibração Linear com Dois Graus de Liberdade $\quad 94$

5.6 Exemplo 6 - Sistema de Suspensão de Veículo 101

5.7 Exemplo 7 - Shear-building Submetido á Explosão 109

5.8 Exemplo 8 - Shear-building com Cinquenta Graus de Liberdade Submetido à Explosão

5.9 Obsevações Finais 126

6 CONCLUSÃO 127

REFERÊNCIAS BIBLIOGRÁFICAS 129 


\section{LISTA DE FIGURAS}

Figura 4.1 - Integração pelo método Newton-Cotes para $n=1,2$

Figura 4.2 - Comparação geométrica entre a regra do trapézio e quadratura de Gauss

Figura 5.1 - Pórtico com um grau de liberdade 61

Figura 5.2 - Exemplo de aplicação de absorvedor de impacto 72

Figura 5.3 - Modelo matemático de um absorvedor de impacto linear 73

Figura 5.4 - Absorvedor de impacto não-linear 78

Figura 5.5 - Isolador de vibração 94

Figura 5.6 - Modelo matemático de um isolador de vibração 95

Figura 5.7 - Modelo de veículo com cinco graus de liberdade 101

Figura 5.8 - Perfil da superfície de rolamento em função do deslocamento horizontal $y$

Figura 5.9 - Shear-building com dez graus de liberdade 109

Figura 5.10 - Shear-building com cinquenta graus de liberdade $\quad 116$ 


\section{LISTA DE TABELAS}

Tabela 4.1 - Fórmulas Newton-Cotes e seus respectivos erros

Tabela 4.2 - Pesos $w$ e argumentos $x$ utilizados nas fórmulas de Gauss-Legendre

Tabela 5.1 - Valores finais das variáveis de projeto para diferentes valores de $\boldsymbol{x}_{0}$.

Tabela 5.2 - Valores finais da função objetivo para diferentes valores de $\boldsymbol{x}_{\mathrm{o}}$.

Tabela 5.3 - Número de minimizações sem restrições para diferentes valores de $\boldsymbol{x}_{\mathrm{o}}$.

Tabela 5.4 - Valores finais das variáveis de projeto para diferentes valores de $\boldsymbol{x}_{\mathrm{o}}$.

Tabela 5.5 - Valores finais da função objetivo para diferentes valores de $\boldsymbol{x}_{\mathrm{o}}$.

Tabela 5.6 - Número de minimizações sem restrições para diferentes valores de $\boldsymbol{x}_{0}$.

Tabela 5.7 - Valores finais das variáveis de projeto para diferentes valores de $\boldsymbol{x}_{\mathrm{o}}$.

Tabela 5.8 - Valores finais da função objetivo para diferentes valores de $\boldsymbol{x}_{0}$.

Tabela 5.9 - Número de minimizações sem restrições para diferentes valores de $\boldsymbol{x}_{0}$.

Tabela 5.10 - Valores finais das variáveis de projeto para diferentes valores de $\boldsymbol{x}_{\mathrm{o}}$.

Tabela 5.11 - Valores finais da função objetivo para diferentes valores de $\boldsymbol{x}_{\mathrm{o}}$.

Tabela 5.12 - Número de minimizações sem restrições para diferentes valores de $\boldsymbol{x}_{\mathrm{o}}$. 
Tabela 5.13 - Valores finais das variáveis de projeto para diferentes valores de $\boldsymbol{x}_{0}$.

Tabela 5.14 - Valores finais da função objetivo para diferentes valores de $\boldsymbol{x}_{\mathrm{o}}$.

Tabela 5.15 - Número de minimizações sem restrições para diferentes valores de $\boldsymbol{x}_{\mathrm{o}}$.

Tabela 5.16 - Valores finais das variáveis de projeto para diferentes valores de $\boldsymbol{x}_{0}$.

Tabela 5.17 - Valores finais da função objetivo para diferentes valores de $\boldsymbol{x}_{0}$.

Tabela 5.18 - Número de minimizações sem restrições para diferentes valores de $\boldsymbol{x}_{\mathrm{o}}$.

Tabela 5.19 - Valores finais das variáveis de projeto para diferentes valores de $\boldsymbol{x}_{\mathrm{o}}$.

Tabela 5.20 - Valores finais da função objetivo para diferentes valores de $\boldsymbol{x}_{0}$.

Tabela 5.21 - Número de minimizações sem restrições para diferentes valores de $\boldsymbol{x}_{0}$.

Tabela 5.22 - Valores finais das variáveis de projeto para diferentes valores de $\boldsymbol{x}_{\mathrm{o}}$.

Tabela 5.23 - Valores finais da função objetivo para diferentes valores de $\boldsymbol{x}_{0}$.

Tabela 5.24 - Número de minimizações sem restrições para diferentes valores de $\boldsymbol{x}_{\mathrm{o}}$.

Tabela 5.25 - Valores finais das variáveis de projeto para diferentes valores de $\boldsymbol{x}_{0}$.

Tabela 5.26 - Valores finais da função objetivo para diferentes valores de $\boldsymbol{x}_{0}$.

Tabela 5.27 - Número de minimizações sem restrições para diferentes valores de $\boldsymbol{x}_{\mathrm{o}}$.

Tabela 5.28 - Valores finais das variáveis de projeto para diferentes valores de $\boldsymbol{x}_{0}$. 
Tabela 5.29 - Valores finais da função objetivo para diferentes valores de $\boldsymbol{x}_{\mathrm{o}}$.

Tabela 5.30 - Número de minimizações sem restrições para diferentes valores de $\boldsymbol{x}_{\mathrm{o}}$.

Tabela 5.31 - Valores finais das variáveis de projeto para diferentes valores de $\boldsymbol{x}_{\mathrm{o}}$.

Tabela 5.32 - Valores finais da função objetivo para diferentes valores de $\boldsymbol{x}_{\mathrm{0}}$.

Tabela 5.33 - Número de minimizações sem restrições para diferentes valores de $\boldsymbol{x}_{\mathrm{o}}$.

Tabela 5.34 - Valores finais das variáveis de projeto para diferentes valores de $\boldsymbol{x}_{\mathrm{o}}$.

Tabela 5.35 - Valores finais da função objetivo para diferentes valores de $\boldsymbol{x}_{0}$.

Tabela 5.36 - Número de minimizações sem restrições para diferentes valores de $\boldsymbol{x}_{\mathrm{o}}$.

Tabela 5.37 - Valores finais das variáveis de projeto para diferentes valores de $\boldsymbol{x}_{\mathrm{o}}$.

Tabela 5.38 - Valores finais da função objetivo para diferentes valores de $\boldsymbol{x}_{\mathrm{o}}$.

Tabela 5.39 - Número de minimizações sem restrições para diferentes valores de $\boldsymbol{x}_{\mathrm{o}}$.

Tabela 5.40 - Valores finais das variáveis de projeto para diferentes valores de $\boldsymbol{x}_{0}$.

Tabela 5.41 - Valores finais da função objetivo para diferentes valores de $\boldsymbol{x}_{\mathrm{o}}$.

Tabela 5.42 - Número de minimizações sem restrições para diferentes valores de $\boldsymbol{x}_{0}$.

Tabela 5.43 - Valores finais das variáveis de projeto para diferentes valores de $\boldsymbol{x}_{\mathrm{o}}$.

Tabela 5.44 - Valores finais da função objetivo para diferentes valores de $\boldsymbol{x}_{\mathrm{0}}$.

Tabela 5.45 - Número de minimizações sem restrições para diferentes 
Tabela 5.46 - Valores finais das variáveis de projeto para diferentes valores de $\boldsymbol{x}_{0}$.

Tabela 5.47 - Valores finais da função objetivo para diferentes valores de $\boldsymbol{x}_{\mathrm{o}}$.

Tabela 5.48 - Número de minimizações sem restrições para diferentes valores de $\boldsymbol{x}_{0}$.

Tabela 5.49 - Valores finais das variáveis de projeto para diferentes valores de $\boldsymbol{x}_{0}$.

Tabela 5.50 - Valores finais da função objetivo para diferentes valores de $\boldsymbol{x}_{0}$.

Tabela 5.51 - Número de minimizações sem restrições para diferentes valores de $\boldsymbol{x}_{\mathrm{o}}$.

Tabela 5.52 - Valores finais das variáveis de projeto para diferentes valores de $\boldsymbol{x}_{\mathrm{o}}$.

Tabela 5.53 - Valores finais da função objetivo para diferentes valores de $\boldsymbol{x}_{0}$.

Tabela 5.54 - Número de minimizações sem restrições para diferentes valores de $\boldsymbol{x}_{\mathrm{o}}$.

Tabela 5.55 - Valores finais das variáveis de projeto para diferentes valores de $\boldsymbol{x}_{0}$.

Tabela 5.56 - Valores finais da função objetivo para diferentes valores de $\boldsymbol{x}_{\mathrm{o}}$.

Tabela 5.57 - Número de minimizações sem restrições para diferentes valores de $\boldsymbol{x}_{\mathrm{o}}$.

Tabela 5.58 - Valores finais das variáveis de projeto para diferentes valores de $\boldsymbol{x}_{0}$.

Tabela 5.59 - Valores finais da função objetivo para diferentes valores de $\boldsymbol{x}_{\mathrm{o}}$.

Tabela 5.60 - Número de minimizações sem restrições para diferentes valores de $\boldsymbol{x}_{0}$.

Tabela 5.61a -Valores finais das variáveis de projeto para diferentes valores de $\boldsymbol{x}_{0}$. 
Tabela 5.61b -Valores finais das variáveis de projeto para diferentes valores de $\boldsymbol{x}_{\mathrm{o}}$.

Tabela 5.62 - Valores finais da função objetivo para diferentes valores de $\boldsymbol{x}_{\mathrm{o}}$.

Tabela 5.63 - Número de minimizações sem restrições para diferentes valores de $\boldsymbol{x}_{\mathrm{o}}$.

Tabela 5.64a -Valores finais das variáveis de projeto para diferentes valores de $\boldsymbol{x}_{0}$.

Tabela 5.64b -Valores finais das variáveis de projeto para diferentes valores de $\boldsymbol{x}_{0}$.

Tabela 5.65 - Valores finais da função objetivo para diferentes valores de $\boldsymbol{x}_{0}$.

Tabela 5.66 - Número de minimizações sem restrições para diferentes valores de $\boldsymbol{x}_{0}$. 


\section{LISTA DE GRÁFICOS}

Gráfico 5.1a - Valores finais da função objetivo para direntes valores de $\boldsymbol{x}_{\mathrm{o}}$ (Tabela 5.2)

Gráfico 5.1b -Valores do deslocamento para diferentes métodos de solução da equação do movimento

Gráfico 5.2 - Número de minimizações sem restrições para diferentes valores de $\boldsymbol{x}_{\mathrm{o}} \quad$ (Tabela 5.3)

Gráfico 5.3 - Valores finais da função objetivo para direntes valores de $\boldsymbol{x}_{\mathrm{o}}$ (Tabela 5.5)

Gráfico 5.4 - Número de minimizações sem restrições para diferentes valores de $\boldsymbol{x}_{\mathrm{o}}$ (Tabela 5.6)

Gráfico 5.5 - Valores finais da função objetivo no caso 1 para diferentes valores de $n$

Gráfico 5.6 - Valores finais da função objetivo para direntes valores de $\boldsymbol{x}_{\mathrm{o}}$ (Tabela 5.8)

Gráfico 5.7 - Número de minimizações sem restrições para diferentes valores de $\boldsymbol{x}_{\mathrm{o}}$ (Tabela 5.9)

Gráfico 5.8 - Valores finais da função objetivo para direntes valores de $\boldsymbol{x}_{\mathrm{o}}$ (Tabela 5.11)

Gráfico 5.9 - Número de minimizações sem restrições para diferentes valores de $\boldsymbol{x}_{\mathrm{o}} \quad$ (Tabela 5.12)

Gráfico 5.10 - Valores finais da função objetivo no caso 1 para diferentes valores de $n$

Gráfico 5.11 -Valores finais da função objetivo para direntes valores de $\boldsymbol{x}_{\mathrm{o}}$ (Tabela 5.14)

Gráfico 5.12 -Número de minimizações sem restrições para diferentes valores de $\boldsymbol{x}_{\mathrm{o}}$ (Tabela 5.15)

Gráfico 5.13 -Valores finais da função objetivo para direntes valores de $\boldsymbol{x}_{\mathrm{o}}$ (Tabela 5.17) 
Gráfico 5.14 -Número de minimizações sem restrições para diferentes valores de $\boldsymbol{x}_{\mathrm{o}}$ (Tabela 5.18)

Gráfico 5.15 - Valores finais da função objetivo no caso 1 para diferentes valores de $n$

Gráfico 5.16 -Valores finais da função objetivo para direntes valores de $\boldsymbol{x}_{0}$ (Tabela 5.20)

Gráfico 5.17 -Número de minimizações sem restrições para diferentes valores de $\boldsymbol{x}_{\mathrm{o}}$ (Tabela 5.21)

Gráfico 5.18 - Valores finais da função objetivo para direntes valores de $\boldsymbol{x}_{0}$ (Tabela 5.23)

Gráfico 5.19 -Número de minimizações sem restrições para diferentes valores de $\boldsymbol{x}_{\mathrm{o}}$ (Tabela 5.24)

Gráfico 5.20 - Valores finais da função objetivo no caso 1 para diferentes valores de $n$

Gráfico 5.21 -Valores finais da função objetivo para direntes valores de $\boldsymbol{x}_{\mathrm{o}}$ (Tabela 5.26)

Gráfico 5.22 -Número de minimizações sem restrições para diferentes valores de $\boldsymbol{x}_{\mathrm{o}}$ (Tabela 5.27)

Gráfico 5.23 - Valores finais da função objetivo para direntes valores de $\boldsymbol{x}_{0}$ (Tabela 5.29)

Gráfico 5.24 -Número de minimizações sem restrições para diferentes valores de $\boldsymbol{x}_{\mathrm{o}}$ (Tabela 5.30)

Gráfico 5.25 - Valores finais da função objetivo no caso 1 para diferentes valores de $n$

Gráfico 5.26 - Valores finais da função objetivo para direntes valores de $\boldsymbol{x}_{\mathrm{o}}$ (Tabela 5.32)

Gráfico 5.27 -Número de minimizações sem restrições para diferentes valores de $\boldsymbol{x}_{\mathrm{o}}$ (Tabela 5.33)

Gráfico 5.28 - Valores finais da função objetivo para direntes valores de $\boldsymbol{x}_{\mathrm{o}}$ (Tabela 5.35)

Gráfico 5.29 -Número de minimizações sem restrições para diferentes valores de $\boldsymbol{x}_{\mathrm{o}}$ (Tabela 5.36)

Gráfico 5.30 - Valores finais da função objetivo no caso 1 para 
Gráfico 5.31 -Valores finais da função objetivo para direntes valores de $\boldsymbol{x}_{\mathrm{o}}$ (Tabela 5.38)

Gráfico 5.32 -Número de minimizações sem restrições para diferentes valores de $\boldsymbol{x}_{\mathrm{o}}$ (Tabela 5.39)

Gráfico 5.33 -Valores finais da função objetivo para direntes valores de $\boldsymbol{x}_{0}$ (Tabela 5.41)

Gráfico 5.34 -Número de minimizações sem restrições para diferentes valores de $\boldsymbol{x}_{\mathrm{o}}$ (Tabela 5.42)

Gráfico 5.35 -Valores finais da função objetivo no caso 1 para diferentes valores de $n$

Gráfico 5.36 - Valores finais da função objetivo para direntes valores de $\boldsymbol{x}_{0}$ (Tabela 5.44)

Gráfico 5.37 -Número de minimizações sem restrições para diferentes valores de $\boldsymbol{x}_{\mathrm{o}}$ (Tabela 5.45)

Gráfico 5.38 -Valores finais da função objetivo para direntes valores de $\boldsymbol{x}_{\mathrm{o}}$ (Tabela 5.47)

Gráfico 5.39 -Número de minimizações sem restrições para diferentes valores de $\boldsymbol{x}_{\mathrm{o}}$ (Tabela 5.48)

Gráfico 5.40 -Valores finais da função objetivo no caso 1 para diferentes valores de $n$

Gráfico 5.41 - Valores finais da função objetivo para direntes valores de $\boldsymbol{x}_{\mathrm{o}}$ (Tabela 5.50)

Gráfico 5.42 -Número de minimizações sem restrições para diferentes valores de $\boldsymbol{x}_{\mathrm{o}}$ (Tabela 5.51)

Gráfico 5.43 -Valores finais da função objetivo para direntes valores de $\boldsymbol{x}_{\mathrm{o}}$ (Tabela 5.53)

Gráfico 5.44 -Número de minimizações sem restrições para diferentes valores de $\boldsymbol{x}_{\mathrm{o}}$ (Tabela 5.54)

Gráfico 5.45 - Valores finais da função objetivo no caso 1 para diferentes valores de $n$

Gráfico 5.46 - Valores finais da função objetivo para direntes valores de $\boldsymbol{x}_{\mathrm{o}}$ (Tabela 5.56) 
Gráfico 5.47 -Número de minimizações sem restrições para diferentes valores de $\boldsymbol{x}_{\mathrm{o}}$ (Tabela 5.57)

Gráfico 5.48 -Valores finais da função objetivo para direntes valores de $\boldsymbol{x}_{\mathrm{o}}$ (Tabela 5.59)

Gráfico 5.49 -Número de minimizações sem restrições para diferentes valores de $\boldsymbol{x}_{\mathrm{o}}$ (Tabela 5.60)

Gráfico 5.50 - Valores finais da função objetivo no caso 1 para diferentes valores de $n$

Gráfico 5.51 - Valores finais da função objetivo para direntes valores de $\boldsymbol{x}_{\mathrm{o}}$ (Tabela 5.62)

Gráfico 5.52 -Número de minimizações sem restrições para diferentes valores de $\boldsymbol{x}_{\mathrm{o}}$ (Tabela 5.63)

Gráfico 5.53 -Valores finais da função objetivo para direntes valores de $\boldsymbol{x}_{\mathrm{o}}$ (Tabela 5.65)

Gráfico 5.54 -Número de minimizações sem restrições para diferentes valores de $\boldsymbol{x}_{\mathrm{o}}$ (Tabela 5.66)

Gráfico 5.55 - Valores finais da função objetivo no caso 1 para diferentes valores de $n$ 


\section{RESUMO}

O Método do Lagrangeano aumentado em problemas de otimização estrutural com restrições dinâmicas, bem como os conceitos matemáticos e numéricos necessários à sua compreensão são descritos. Este método resolve uma seqüência de problemas de minimização sem restrições definidos utilizando a função objetivo e as funções restrições. Um programa de computador é desenvolvido e aplicado em diversos exemplos. Alem disto, foi efetuada uma análise de sensibilidade com relação aos parâmetros utilizados no método. O método mostrou-se eficiente nas aplicações em problemas com restrições dinâmicas.

\section{"ABSTRACT"}

We present the method of the augmented Lagrangian in problems of scructural optimization with dynamic restrictions, as well as its mathemathical and numerical concepts. This method solves a series of unconstrained minimization problems using the objective function and the restriction functions. A computational program is implemented and applied in several examples. The augmented Lagrangian parameters senbility is analysed. The method is quite efficient in applications in optimization problems with dynamic restrictions. 


\section{Capítulo 1}

\section{INTRODUÇÃO}

O grande avanço da tecnologia e o aumento da competitividade exigem, cada vez mais, projetos arrojados. Processos de otimização são amplamente utilizados para desenvolver soluções que exploram ao máximo a capacidade de trabalho das estruturas. Neste contexto, a pesquisa de algoritmos adequados para se chegar mais facilmente à solução de determinados grupos de problemas, passa a ser relevante. Existem diversos algoritmos de otimização destinados a tratar uma variada gama de problemas de engenharia.

O Método dos Multiplicadores, também conhecido como Método do Lagrangeano Aumentado, desenvolvido na década de 60, encontra-se amplamente descrito na literatura como por exemplo em Luemberger [5] e Fletcher [25]. O método foi recentemente aplicado a problemas de otimização com restrições dinâmicas por Chahande e Arora [1], Goldenberg, Silva, Pimenta e Brasil [17], além de outros. 
O presente trabalho é dedicado ao estudo deste método com aplicações em otimização estrutural e mecânica com restrições dinâmicas. Inicialmente decreve-se o método, bem como os conceitos matemáticos, numéricos e computacionais envolvidos. E, posteriormente, diversas aplicações em engenharia mecânica e de estruturas são analisadas. Mais especificamente o trabalho é desenvolvido conforme descrito abaixo.

Todo o embasamento matemático necessário para o entendimento do método são descritos no capítulo 2. Neste capítulo são definidos conceitos relativos à matrizes, funções de várias variáveis reais, equações diferenciais e programação matemática.

A otimização estrutural com restrições dinâmicas é analisada no capítulo 3 , onde é apresentado o método do Lagrangeano aumentado e um algoritmo implementável. Este método pode ser implementado de diversas formas. Esta diversificação é resultado de diferentes combinações de métodos numéricos para a integração numérica, interpolação polinomial e integração de equações diferenciais.

Os métodos numéricos envolvidos na programação do método do Lagrangeano aumentado são detalhados no capítulo 4. Nas seções 4.1 à 4.4 são tratados a solução de sistemas lineares, a integração numérica, interpolação polinomial e métodos de integração direta para problemas de dinâmica estrutural. Na seção 4.5 é estudado um algoritmo de minimização sem restrições e na 4.6 o método das diferenças finitas.

Além disso foi efetuada a implementação computacional do método. Para tanto programas em linguagem $\mathrm{C}$ foram desenvolvidos e testados em diversos problemas dinâmicos estruturais e mecânicos.

No capítulo 5, diversos exemplos são analisados com modelos que variam de elásticolinear à viscoelastoplásticos. Os exemplos 5.1 e 5.2 tratam do projeto ótimo de um pórtico com um grau de liberdade. Já os exemplos 5.3 e 5.4 são o dimensionamento ótimo de um amortecedor de impacto. Um isolador de vibração é dimensionado no exemplo 5.5. O sistema de suspensão de um veículo é projetado no exemplo 5.6. A análise estrutural de grande porte é realizada nos exemplos 5.7 e 5.8, onde edifícios submetidos a esforços de explosão são dimensionados.

No capítulo 6 é apresentado a conclusão, enquanto que no capítulo 7 as referências bibliográficas. 


\section{Capítulo 2}

\section{CONCEITOS MATEMÁTICOS BÁSICOS}

Neste capítulo são descritos os conceitos matemáticos básicos necessários ao entendimento do método do Lagrangeano aumentado. Os assuntos aqui tratados são:

- Espaços Vetoriais;

- Espaços Vetoriais Euclidianos e Espaços Normados;

- Funções de Várias Variáveis Reais;

- Seqüências;

- Funcionais;

- Equações Diferenciais Ordinárias;

- Programação Matemática:

Otimização sem Restrições;

Otimização com Restrições.

O leitor já familiarizado com estes conceitos pode continuar a leitura no capítulo 3 página 25. 


\subsection{ESPAÇOS VETORIAIS}

$\mathrm{Na}$ geometria e na física entra-se em contato com grandezas denominadas vetores, designados aqui por $\boldsymbol{a}, \boldsymbol{b}, \ldots$, para os quais são definidos as operações de adição, produto por um escalar, entre outras.

\section{Definição 2.1}

Denomina-se espaço vetorial a todo conjunto $H$ de elementos $\boldsymbol{a}, \boldsymbol{b}, \ldots$, denominados vetores, tal que:

a) a cada par de elementos $\boldsymbol{a}$ e $\boldsymbol{b}$ de $\mathrm{H}$ fica associado um único elemento $\boldsymbol{a}+\boldsymbol{b}$ de $\mathrm{H}$, denominado soma de $\boldsymbol{a} \operatorname{com} \boldsymbol{b}$, de modo que:

1) $\boldsymbol{a}+\boldsymbol{b}=\boldsymbol{b}+\boldsymbol{a}, \quad \forall \boldsymbol{a}, \boldsymbol{b} \in \mathrm{H}$

2) $(\boldsymbol{a}+\boldsymbol{b})+\boldsymbol{c}=\boldsymbol{a}+(\boldsymbol{b}+\boldsymbol{c}), \quad \forall \boldsymbol{a}, \boldsymbol{b}, \boldsymbol{c} \in \mathrm{H}$;

3) $\exists \mathbf{0} \in \mathrm{H} \mid \boldsymbol{a}+\mathbf{0}=\boldsymbol{a}, \quad \forall \boldsymbol{a} \in \mathrm{H}$;

4) $\exists(-\boldsymbol{a}) \in \mathrm{H} \mid \boldsymbol{a}+(-\boldsymbol{a})=\mathbf{0}, \quad \forall \boldsymbol{a} \in \mathrm{H}$;

b) a um número real $\alpha$ e a um elemento $\boldsymbol{a}$ de $\mathrm{H}$ fica associado um e só um elemento de $\mathrm{H}$, indicado por $\alpha \boldsymbol{a}$, denominado produto de $\boldsymbol{a}$ pelo escalar $\alpha$, de modo que:

1) $(\alpha+\beta) \boldsymbol{a}=\alpha \boldsymbol{a}+\beta \boldsymbol{a}, \quad \forall \alpha, \beta \in \mathbf{R}, \forall \boldsymbol{a} \in \mathrm{H}$;

2) $\alpha(\boldsymbol{a}+\boldsymbol{b})=\alpha \boldsymbol{a}+\alpha \boldsymbol{b}, \quad \forall \alpha \in \mathbf{R}, \forall \boldsymbol{a}, \boldsymbol{b} \in \mathrm{H}$;

3) $\alpha(\beta \boldsymbol{a})=\beta(\alpha \boldsymbol{a})=(\alpha \beta) \boldsymbol{a}, \quad \forall \alpha, \beta \in \mathbf{R}, \forall \boldsymbol{a} \in \mathrm{H}$;

4) $1 \boldsymbol{a}=\boldsymbol{a}, \quad \forall \boldsymbol{a} \in \mathrm{H}$.

São exemplos de espaços vetoriais:

a) O conjunto dos números reais: $\mathbf{R}$;

b) Os produtos cartesianos de $\mathbf{R}: \mathbf{R}^{n}=\mathbf{R} \times \mathbf{R} \times \ldots \times \mathbf{R}$, ou seja, o conjunto das ênuplas $\left(a_{1}, a_{2}, \ldots, a_{n}\right)$, aqui representados por $\boldsymbol{a}=\left[a_{i}\right]$, onde $a_{i} \in \mathbf{R}, i=1, \ldots, n$;

c) $\mathrm{O}$ conjunto $\mathbf{M}(m \times n)$ de matrizes de dimensão ( $m \times n$ ), aqui representados por $\boldsymbol{M}=\left[M_{i j}\right]$, onde $M_{i j} \in \mathbf{R}, i=1, \ldots, m$ e $j=1, \ldots, \mathrm{n}$;

d) $\mathrm{O}$ conjunto dos números complexos: $\mathbf{C}$. 
Definição 2.2

Diz-se que os vetores $\left\{\boldsymbol{x}_{1}, \boldsymbol{x}_{2}, \ldots, \boldsymbol{x}_{n}\right\}$ são linearmente independentes (LI) se, e somente se

$$
a_{1} \boldsymbol{x}_{1}+a_{2} \boldsymbol{x}_{2}+\ldots+a_{n} \boldsymbol{x}_{n}=\mathbf{0} \Rightarrow a_{1}=a_{2}=\ldots=a_{n}=0
$$

Caso contrário eles se dizem linearmente dependentes (LD).

\section{Definição 2.3}

Diz-se que um espaço vetorial $\mathrm{H}$ tem dimensão $n$ finita quando nele existem $n$ vetores linearmente independentes e quaisquer $n+1$ vetores forem linearmente dependentes. Caso contrário, diz-se que a dimensão de $\mathrm{H}$ é infinita.

A dimensão de $\mathbf{R}$ é 1 e a dimensão de $\mathbf{R}^{n}$ é $n$.

Definição 2.4

Um conjunto de $n$ vetores linearmente independentes pertencentes a um espaço vetorial $\mathrm{H}$ de dimensão finita $n$ forma uma base de $\mathrm{H}$.

Considere-se $\boldsymbol{y} \in \mathrm{H}$ e a base $\left\{\boldsymbol{e}_{1}, \boldsymbol{e}_{2}, \boldsymbol{e}_{3}\right\}$ de H. Como $\left\{\boldsymbol{y}, \boldsymbol{e}_{1}, \boldsymbol{e}_{2}, \boldsymbol{e}_{3}\right\}$ são linearmente dependentes, pode-se escrever

$$
a \boldsymbol{y}+a^{1} \boldsymbol{e}_{1}+a^{2} \boldsymbol{e}_{2}+a^{3} \boldsymbol{e}_{3}=\mathbf{0}, a \neq 0
$$

Assim, fazendo-se $y^{i}=-a^{i} / a$ para $(i=1,2,3)$, tem-se que

$$
\boldsymbol{y}=y^{1} \boldsymbol{e}_{1}+y^{2} \boldsymbol{e}_{2}+y^{3} \boldsymbol{e}_{3} .
$$

\section{Definição 2.5}

Seja $\boldsymbol{y}$ um vetor de $\mathrm{H}$ e seja $\left\{\boldsymbol{e}_{1}, \boldsymbol{e}_{2}, \boldsymbol{e}_{3}\right\}$ uma base de H. Quando se escreve

$$
\boldsymbol{y}=\sum_{i=1}^{3} y^{i} \boldsymbol{e}_{i},
$$

os números reais $y^{i}, i=1,2,3$, são denominados componentes de $\boldsymbol{y}$ na base $\left\{\boldsymbol{e}_{1}, \boldsymbol{e}_{2}, \boldsymbol{e}_{3}\right\}$. 


\subsection{ESPAÇOS VETORIAIS EUCLIDIANOS E ESPAÇOS NORMADOS}

Definição 2.6

Um espaço vetorial $\mathbf{H}$ com produto escalar ou produto interno é um espaço vetorial munido de uma aplicação de $\mathrm{H} \times \mathrm{H}$ em $\mathbf{R}$, que associa a cada par de vetores $\boldsymbol{x}, \boldsymbol{y} \in \mathrm{H}$ um e só um número real $\boldsymbol{x} \cdot \boldsymbol{y}$ verificando as seguintes propriedades:

1) $x \cdot y=y \cdot x, \quad \forall \boldsymbol{x}, \boldsymbol{y} \in \mathrm{H}$;

2) $(x+y) \cdot z=x \cdot z+y \cdot z, \quad \forall x, y, z \in \mathrm{H}$

3) $\alpha(x . y)=\alpha x . y, \quad \forall \alpha \in \mathbf{R}, \forall x, y \in H$;

4) $\boldsymbol{x} . \boldsymbol{x} \geq 0, \quad \forall \boldsymbol{x} \in \mathrm{H}$, sendo que $\boldsymbol{x} \cdot \boldsymbol{x}=0$ somente quando $\boldsymbol{x}=\mathbf{0}$.

É comum, especialmente no contexto de funções, utilizar a notação $\langle\boldsymbol{x}, \boldsymbol{y}\rangle$ para o produto escalar.

Definição 2.7

Em $\mathbf{R}^{n}$ o produto escalar de $\boldsymbol{x}$ por $\boldsymbol{y}$ é definido por:

$$
\langle\boldsymbol{x}, \boldsymbol{y}\rangle=\left(x_{1}, x_{2}, \ldots, x_{n}\right) \cdot\left(y_{1}, y_{2}, \ldots, y_{n}\right) \stackrel{\ominus}{=} \sum_{i=1}^{n} x_{i} y_{i}
$$

O sinal " $="$ significa "igual por definição" e será utilizado diversas vezes neste texto.

\section{Definição 2.8}

Um espaço vetorial de dimensão finita dotado de produto escalar é denominado espaço vetorial Euclidiano.

Definição 2.9

Seja $\mathrm{H}$ um espaço vetorial. Diz-se que $\|\cdot\|: H \mapsto \mathbf{R}$ é uma norma se

a) $\|\alpha \boldsymbol{x}\|=\mid \alpha\|\boldsymbol{x}\|, \quad \forall \alpha \in \mathbf{R}, \quad \forall \boldsymbol{x} \in \mathrm{H}$

b) $\|\boldsymbol{x}\| \geq 0, \quad \forall \boldsymbol{x} \in \mathrm{H}, \quad\|\boldsymbol{x}\|=0 \Leftrightarrow \boldsymbol{x}=\mathbf{0}$

c) $\|x+y\| \leq\|x\|+\|y\|, \quad \forall x, y \in H$

Definição 2.10

A norma euclidiana $\|\boldsymbol{x}\|$ ou magnitude de um vetor $\boldsymbol{x}$ pertencente a um espaço vetorial Euclidiano é definida por $\|\boldsymbol{x}\|=\sqrt{\langle\boldsymbol{x}, \boldsymbol{x}\rangle}$. 
Um vetor cuja magnitude é igual a 1 é denominado vetor unitário ou versor.

Para o espaço vetorial $\mathbf{R}^{n}$ a expressão

$$
\|\boldsymbol{x}\|_{p}=\sqrt[p]{\left|x_{1}\right|^{p}+\left|x_{2}\right|^{p}+\ldots+\left|x_{n}\right|^{p}}, \quad \boldsymbol{x} \in \mathbf{R}^{n}
$$

define uma família de normas, à qual pertencem as seguintes normas

$$
\begin{aligned}
& \|\boldsymbol{x}\|_{1}=\left|x_{1}\right|+\left|x_{2}\right|+\ldots+\left|x_{n}\right|, \\
& \|\boldsymbol{x}\|_{2}=\sqrt{x_{1}^{2}+x_{2}^{2}+\ldots+x_{n}^{2}}, \\
& \|\boldsymbol{x}\|_{\infty}=\max _{1 \leq i \leq n}\left|x_{i}\right| .
\end{aligned}
$$

Definição 2.11

A distância entre dois vetores $\boldsymbol{a}$ e $\boldsymbol{b}$ de um espaço Euclidiano é definida por:

$$
d(\boldsymbol{a}, \boldsymbol{b}) \stackrel{\ominus}{=}\|(\boldsymbol{b}-\boldsymbol{a})\|
$$

Definição 2.12

O ângulo $\theta$ entre dois vetores $\boldsymbol{x}, \boldsymbol{y}$ é definido por:

$$
\theta \stackrel{\ominus}{=} \arccos \left(\frac{\langle\boldsymbol{x}, \boldsymbol{y}\rangle}{\|\boldsymbol{x}\|\|\boldsymbol{y}\|}\right) .
$$

Definição 2.13

Dois vetores $\boldsymbol{x}, \boldsymbol{y}$ são ortogonais, isto é, $\boldsymbol{x} \perp \boldsymbol{y}$, se $\theta=90^{\circ}$ ou $\langle\boldsymbol{x}, \boldsymbol{y}\rangle=0$.

\section{Definição 2.14}

Uma base $\left\{\boldsymbol{e}_{1}, \boldsymbol{e}_{2}, \boldsymbol{e}_{3}\right\}$ é dita ortonormal se $\left\|\boldsymbol{e}_{i}\right\|=1$ e $\boldsymbol{e}_{i} \perp \boldsymbol{e}_{j}(i \neq j)$, ou seja,

$$
\left\langle\boldsymbol{e}_{i}, \boldsymbol{e}_{j}\right\rangle=\left\{\begin{array}{ll}
0 & \text { se } i \neq j \\
1 & \text { se } i=j
\end{array} .\right.
$$


Introduzindo-se o símbolo de Kronecker $\delta_{i j}$,

$$
\delta_{i j} \stackrel{\diamond}{=}\left\{\begin{array}{ll}
0 & \text { se } i \neq j \\
1 & \text { se } i=j
\end{array},\right.
$$

pode-se escrever (2.4) da seguinte maneira:

$$
\left\langle\boldsymbol{e}_{i}, \boldsymbol{e}_{j}\right\rangle=\delta_{i j}
$$

A matriz quadrada correspondente a $\delta_{i j}$ é a matriz identidade $(3 \times 3)$, pois

$$
\left[\delta_{i j}\right]=\left[\begin{array}{lll}
1 & 0 & 0 \\
0 & 1 & 0 \\
0 & 0 & 1
\end{array}\right]=[\boldsymbol{I}] .
$$

Definição 2.15

A matriz transposta de $\boldsymbol{A}$, indicada por $\boldsymbol{A}^{T}$, é definida por:

$$
A_{i j}^{T}=A_{j i}, \quad i, j=1, \ldots, n .
$$

Definição 2.16

Uma matriz $\boldsymbol{A}$ é simétrica se $\boldsymbol{A}=\boldsymbol{A}^{T}$, isto é, $A_{i j}=A_{j i}, i, j=1, \ldots, n$.

Definição 2.17

Uma matriz $\boldsymbol{A}$ é definida positiva se ela for simétrica e $\langle\boldsymbol{x}, \boldsymbol{A x}\rangle>0$ para todo $\boldsymbol{x} \neq \mathbf{0}$.

\subsection{FUNÇÕES DE VÁRIAS VARIÁVEIS REAIS}

\section{Definição 2.18}

Denomina-se vizinhança aberta ou esfera aberta de centro $\boldsymbol{y} \in \mathbf{R}^{n}$ e raio $r>0$ ao conjunto $B_{a}(\boldsymbol{y}, r)$ definido por

$$
B_{a}(\boldsymbol{y}, r)=\left\{\boldsymbol{x} \in \mathbf{R}^{n} \mid\|\boldsymbol{x}-\boldsymbol{y}\|<r\right\}
$$


Definição 2.19

Denomina-se vizinhança fechada ou esfera fechada de centro $\boldsymbol{y} \in \mathbf{R}^{n} \mathrm{e}$ raio $r>0$ ao conjunto $B_{f}(\boldsymbol{y}, r)$ definido por

$$
B_{f}(\boldsymbol{y}, r)=\left\{\boldsymbol{x} \in \mathbf{R}^{n} \mid\|\boldsymbol{x}-\boldsymbol{y}\| \leq r\right\}
$$

Definição 2.20

Diz-se que um conjunto $\Gamma$ em $\mathbf{R}^{n}$ é aberto se para cada $\boldsymbol{y} \in \Gamma$ existir um número real $r>0$ tal que

$$
\Gamma \supset B_{a}(\boldsymbol{y}, r) .
$$

Definição 2.21

Diz-se que um conjunto $\Gamma$ em $\mathbf{R}^{n}$ é fechado se e somente se seu complementar for aberto em $\mathbf{R}^{n}$.

\section{Definição 2.22}

Diz-se que um conjunto $\Gamma$ em $\mathbf{R}^{n}$ é limitado se existir um número real $b>0$ e um ponto $\boldsymbol{y} \in \mathbf{R}^{n}$ tal que

$$
d(\boldsymbol{x}, \boldsymbol{y})=\|\boldsymbol{x}-\boldsymbol{y}\| \leq b,
$$

para todo $\boldsymbol{x} \in \Gamma$.

Definição 2.23

Diz-se que um conjunto $\Gamma$ em $\mathbf{R}^{n}$ é compacto se for limitado e fechado.

No que segue, considerar-se-á funções $f: \Omega \subset \mathbf{R}^{n} \rightarrow \mathbf{R}$.

\section{Definição 2.24}

Diz-se que uma função $f(\boldsymbol{x})$ é contínua em $\boldsymbol{x}$ se dado um $\varepsilon>0$ existe um $\delta>0$ tal que $\|\boldsymbol{y}-\boldsymbol{x}\|<\delta$ implica em $\|f(\boldsymbol{y})-f(\boldsymbol{x})\|<\varepsilon$.

\section{Definição 2.25}

Seja $\Gamma$ um conjunto aberto de $\mathbf{R}^{n}$ e seja $\overline{\boldsymbol{x}} \in \Gamma$. Diz-se que $f: \Gamma \rightarrow \mathbf{R}$ possui derivadas parciais em $\overline{\boldsymbol{x}}$ em relação a $x_{i}, i=1, \ldots, n$, se

$$
\frac{f\left(\bar{x}_{1}, \ldots, \bar{x}_{i-1}, \bar{x}_{i}+\delta, \bar{x}_{i+1}, \ldots, \bar{x}_{n}\right)-f\left(\bar{x}_{1}, \ldots, \bar{x}_{n}\right)}{\delta}
$$


possuir limite quando $\delta \rightarrow 0$. Este limite é denominado derivada parcial de $f(\boldsymbol{x})$ em relação a $x_{i}$ em $\overline{\boldsymbol{x}}$ e é denotada por $\frac{\partial f(\overline{\boldsymbol{x}})}{\partial x_{i}}$.

Indicar-se-á por $C_{k}(\Omega)$ o conjunto das funções $f: \Omega \subset \mathbf{R}^{n} \rightarrow \mathbf{R}$ com derivadas parciais contínuas até ordem $k$, onde $\Omega$ é um conjunto aberto em $\mathbf{R}^{n}$.

Indicar-se-á por $C_{k}[\Gamma]$ o conjunto das funções $f: \Gamma \subset \mathbf{R}^{n} \rightarrow \mathbf{R}$ com derivadas parciais contínuas até ordem $k$, onde $\Gamma$ é um conjunto fechado em $\mathbf{R}^{n}$.

Fato 2.1:

O conjunto $C_{k}(\Omega)$ com as operações usuais de soma e multiplicação por um escalar é um espaço vetorial.

Fato 2.2:

O conjunto $C_{k}[\Gamma]$ com as operações usuais de soma e multiplicação por um escalar é um espaço vetorial.

Nas definições seguintes considera-se que $f:(a, b) \subset \mathbf{R} \rightarrow \mathbf{R}$ e suas derivadas até ordem $k$ assumem sempre valores finitos em $(\mathrm{a}, \mathrm{b})$.

Definição 2.26

Em $C_{0}(a, b),(\mathrm{a}, \mathrm{b}) \subset \mathbf{R}$, define-se o produto escalar das funções $f$ e $g$ por:

$$
\langle f, g\rangle=\int_{a}^{b} f(x) g(x) d x
$$

Definição 2.27

Em $C_{0}[a, b],[\mathrm{a}, \mathrm{b}] \subset \mathbf{R}$, define-se o produto escalar das funções $f$ e $g$ por:

$$
\langle f, g\rangle=\int_{a}^{b} f(x) g(x) d x
$$




\section{Definição 2.28}

$\mathbf{L}_{2}(a, b)$ é o espaço vetorial das funções $f:(a, b) \mapsto \mathbf{R}$, para os quais a integral

$$
\int_{a}^{b} f^{2}(x) d x
$$

existe e é finita. Uma norma em $\mathbf{L}_{2}(a, b)$ é

$$
\|f\|_{2}=\sqrt{\int_{a}^{b} f^{2}(x) d x} .
$$

$\mathrm{O}$ espaço $\mathbf{L}_{2}(a, b)$ contém, por exemplo, funções contínuas em $(a, b)$ e funções descontínuas em um número finito de pontos mas com a integral (2.15) finita.

\section{Definição 2.29}

A norma natural de $C_{k}(a, b)$ é

$$
\|f\|=\max _{a<x<b}\left(|f(x)|+\left|f^{\prime}(x)\right|+\ldots+\left|f^{k}(x)\right|\right) .
$$

Outras normas para o conjunto de funções $C_{0}(a, b)$ são:

$$
\begin{aligned}
& \|f\|_{1}=\int_{a}^{b}|f(x)| d x \\
& \|f\|_{\infty}=\max _{a<x<b}|f(x)|
\end{aligned}
$$

Observa-se que (2.19) é um caso particular de (2.17), ou seja, para $k=0$.

\section{Definição 2.30}

A distância entre as funções $f, g \in \mathrm{H}$ é definida por

$$
d(f, g)=\|f-g\|
$$

\section{Definição 2.31}

Denomina-se vizinhança aberta da função $f$ de raio $r>0$ ao conjunto $B_{a}(f, r)$ definido por

$$
B_{a}(f, r)=\{g \mid d(f, g)<r\} .
$$


Considerando-se que a função $f(\boldsymbol{x}) \in C_{2}(\Omega)$ pode-se apresentar as definições $2.33 \mathrm{e}$ 2.34 .

Definições 2.32

O vetor das derivadas primeiras parciais de $f(\boldsymbol{x})$ em um ponto $\boldsymbol{x}$, denomina-se vetor gradiente, e será denotado por:

$$
\nabla f(\boldsymbol{x})=\left[\begin{array}{c}
\frac{\partial f}{\partial x_{1}} \\
\partial f \\
\frac{\partial x_{2}}{\vdots} \\
\frac{\partial f}{\partial x_{n}}
\end{array}\right] .
$$

onde $\nabla$ indica o operador diferencial gradiente $\nabla=\left[\frac{\partial}{\partial x_{i}}\right]$.

Definição 2.33

Denomina-se matriz Hessiana da função $f(\boldsymbol{x})$ à matriz

$$
\nabla^{2} f(\boldsymbol{x}) \stackrel{\diamond}{=}\left[\frac{\partial^{2} f}{\partial x_{i} \partial x_{j}}\right]
$$

\section{Definição 2.34}

Seja $f(\boldsymbol{x})$ uma função a valores reais de um conjunto aberto $\Gamma \subset \mathbf{R}^{n}$. Se existir o gradiente $\nabla f(\boldsymbol{x})$ para todo $\overline{\boldsymbol{x}} \in \Gamma$, então se diz que a função $f(\boldsymbol{x})$ é diferenciável.

\section{Definição 2.35}

Uma reta em $\mathbf{R}^{n}$ é um conjunto de pontos

$$
\boldsymbol{x}(\alpha)=\boldsymbol{x}+\alpha \boldsymbol{d}
$$

para todo $\alpha \in \mathbf{R}$, onde $\overline{\boldsymbol{x}} \in \mathbf{R}^{n}$ é um ponto fixo e $\boldsymbol{d} \in \mathbf{R}^{n}$ é a direção da reta.

\section{Definição 2.36}

A derivada da função $f(\boldsymbol{x})$ ao longo de uma reta $\boldsymbol{x}(\alpha)$ é dada por

$$
\frac{d f}{d \alpha}=\sum_{i=1}^{n} \frac{d x_{i}(\alpha)}{d \alpha} \frac{\partial f}{\partial x_{i}}=\sum_{i=1}^{n} d_{i} \frac{\partial f}{\partial x_{i}}
$$


Decorre das definições anteriores que

$$
\frac{d f}{d \alpha}=\langle\boldsymbol{d}, \nabla f\rangle
$$

e $\frac{d f}{d \alpha}$ é denominada inclinação de $f(\boldsymbol{x})$ ao longo da reta $\boldsymbol{x}(\alpha)$.

Definição 2.37

Se $\boldsymbol{x}^{1}, \boldsymbol{x}^{2} \in \mathbf{R}^{n}$, então a reta passando por $\boldsymbol{x}^{1}$ e $\boldsymbol{x}^{2}$ é definida como sendo o conjunto dos pontos

$$
\left\{\boldsymbol{x} \in \mathbf{R}^{n} \mid \boldsymbol{x}=\boldsymbol{x}^{1}+\lambda\left(\boldsymbol{x}^{2}-\boldsymbol{x}^{1}\right), \lambda \in \mathbf{R}\right\}
$$

Definição 2.38

O segmento de reta que liga $\boldsymbol{x}^{1}$ e $\boldsymbol{x}^{2}$ é definido pelo conjunto de pontos

$$
\left\{\boldsymbol{x} \in \mathbf{R}^{n} \mid \boldsymbol{x}=\boldsymbol{x}^{1}+\lambda\left(\boldsymbol{x}^{2}-\boldsymbol{x}^{1}\right), 0 \leq \lambda \leq 1\right\} .
$$

Definição 2.39

O conjunto não vazio $X$ em $\mathbf{R}^{n}$ é convexo se para dois pontos quaisquer $\boldsymbol{x}^{1}, \boldsymbol{x}^{2} \in X$, o segmento de reta que une $\boldsymbol{x}^{1}$ e $\boldsymbol{x}^{2}$ estiver contido em $X$, isto é,

$$
\lambda \boldsymbol{x}^{1}+(1-\lambda) \boldsymbol{x}^{2} \in X \text { para todo } 0 \leq \lambda \leq 1 .
$$

\section{Definição 2.40}

Uma função $f(\boldsymbol{x})$ a valores reais definida em um conjunto convexo $X$ de $\mathbf{R}^{n}$ diz-se convexa se para dois pontos quaisquer $\boldsymbol{x}^{1}, \boldsymbol{x}^{2} \in X$ e qualquer $\lambda$ real, $0 \leq \lambda \leq 1$, tem-se

$$
f\left(\lambda x^{1}+(1-\lambda) x^{2}\right) \leq \lambda f\left(x^{1}\right)+(1-\lambda) f\left(x^{2}\right) .
$$

Se a desigualdade acima for estrita para todo $\boldsymbol{x}^{1} \neq \boldsymbol{x}^{2}$ em $X$ e qualquer $0<\lambda<1$ então, diz-se que $f(x)$ é estritamente convexa. 
Nas definições 2.41 à 2.44 considerar-se-á funções $f: \Gamma \subset \mathbf{R}^{n} \rightarrow \mathbf{R}$.

\section{Definição 2.41}

Diz-se que um ponto $\boldsymbol{x}^{*} \in \Gamma$ é um ponto de mínimo local ou ponto de mínimo relativo de $f(\boldsymbol{x})$ se numa vizinhança aberta de $\boldsymbol{x}^{*}, B_{a}\left(\boldsymbol{x}^{*}, r\right)$, $f(\boldsymbol{x}) \geq f\left(\boldsymbol{x}^{*}\right)$ para todo $\boldsymbol{x} \in B_{a}\left(\boldsymbol{x}^{*}, r\right)$.

Definição 2.42

Diz-se que um ponto $x^{*} \in \Gamma$ é um ponto de mínimo global de $f(x)$ se $f(\boldsymbol{x}) \geq f\left(\boldsymbol{x}^{*}\right)$ para todo $\boldsymbol{x} \in \Gamma$. Se $f(\boldsymbol{x})>f\left(\boldsymbol{x}^{*}\right)$ para todo $\boldsymbol{x} \in \Gamma, \boldsymbol{x} \neq \boldsymbol{x}^{*}$, diz-se que $x^{*}$ é um ponto de mínimo global estrito de $f(x)$.

\section{Definição 2.43}

Diz-se que um ponto $\boldsymbol{x}^{*} \in \Gamma$ é um ponto de máximo local de $f(\boldsymbol{x})$ se numa vizinhança aberta de $\boldsymbol{x}^{*}, B_{a}\left(\boldsymbol{x}^{*}, r\right), f(\boldsymbol{x}) \leq f\left(\boldsymbol{x}^{*}\right)$ para todo $\boldsymbol{x} \in$ $B_{a}\left(\boldsymbol{x}^{*}, r\right)$.

\section{Definição 2.44}

Diz-se que um ponto $\boldsymbol{x}^{*} \in \Gamma$ é um ponto de máximo global de $f(\boldsymbol{x})$ se $f(\boldsymbol{x}) \leq f\left(\boldsymbol{x}^{*}\right)$ para todo $\boldsymbol{x} \in \Gamma$. Se $f(\boldsymbol{x})<f\left(\boldsymbol{x}^{*}\right)$ para todo $\boldsymbol{x} \in \Gamma, \boldsymbol{x} \neq \boldsymbol{x}^{*}$, diz-se que $x^{*}$ é um ponto de máximo global estrito de $f(\boldsymbol{x})$.

\section{Observações:}

2.1 Funções convexas possuem pelo menos um ponto de mínimo global. Quando estas funções são estritamente convexas este ponto não só existe mas é único.

2.2 $f\left(x^{*}\right)$ é denominado extremo da função $f(x)$ e o ponto $x^{*}$ extremante. 


\subsection{SEQUÊNCIAS}

Definição 2.45

Seja

$$
\left\{\boldsymbol{u}_{k}\right\}=\left\{\boldsymbol{u}_{1}, \boldsymbol{u}_{2}, \ldots, \boldsymbol{u}_{k}, \ldots\right\}
$$

uma sequência de elementos de $\mathrm{H}$. Diz-se que a sequência $\left(\boldsymbol{u}_{k}\right)$ é convergente para $\boldsymbol{u}^{*} \in \mathrm{H}$ se $d\left(\boldsymbol{u}_{k}, \boldsymbol{u}^{*}\right) \rightarrow 0$ quando $k \rightarrow \infty$. Escreve-se, então que

$$
\lim _{k \rightarrow \infty} \boldsymbol{u}_{k}=\boldsymbol{u}^{*}
$$

Definição 2.46

Define-se como taxa de convergência da sequência $\left\{\boldsymbol{u}_{k}\right\}=\left\{\boldsymbol{u}_{1}, \boldsymbol{u}_{2}, \ldots, \boldsymbol{u}_{k}, \ldots\right\}, \boldsymbol{u}_{k} \in \mathrm{H}$, a razão

$$
q=\lim _{k \rightarrow \infty} \frac{\left\|\boldsymbol{u}_{k}-\boldsymbol{u} *\right\|}{\left\|\boldsymbol{u}_{k+1}-\boldsymbol{u} *\right\|}
$$

Definição 2.47

Diz-se que a convergência da sequência $\left\{\boldsymbol{u}_{k}\right\}=\left\{\boldsymbol{u}_{1}, \boldsymbol{u}_{2}, \ldots, \boldsymbol{u}_{k}, \ldots\right\}, \boldsymbol{u}_{k} \in$ $\mathrm{H}$, é linear, ou de primeira ordem, se

$$
\left\|\boldsymbol{u}_{k+1}-\boldsymbol{u}^{*}\right\| \leq \beta\left\|\boldsymbol{u}_{k}-\boldsymbol{u}^{*}\right\|, \beta>0 .
$$

Definição 2.48

Diz-se que a convergência da sequência $\left\{\boldsymbol{u}_{k}\right\}=\left\{\boldsymbol{u}_{1}, \boldsymbol{u}_{2}, \ldots, \boldsymbol{u}_{k}, \ldots\right\}, \boldsymbol{u}_{k} \in$ $\mathrm{H}$, é quadrática, ou de segunda ordem, se

$$
\left\|\boldsymbol{u}_{k+1}-\boldsymbol{u}^{*}\right\| \leq \beta\left\|\boldsymbol{u}_{k}-\boldsymbol{u}^{*}\right\|^{2}, \beta>0 .
$$

\section{$2.5 \quad$ FUNCIONAIS}


Procurar-se-á nesta apresentação manter um certo paralelismo entre o cálculo variacional e o cálculo diferencial clássico.

Definição 2.49

Seja $B$ um espaço vetorial de funções. Chama-se funcional a aplicação $\Pi$ que associa a cada elemento $f$ de $B$ um único elemento $y$ de $\mathbf{R}$. A notação utilizada é $\Pi: B \mapsto \mathbf{R}$, tal que se $f \in B$ então $y=\Pi(f)$.

\section{Definição 2.50}

Um funcional $\Pi: B \mapsto \mathbf{R}$ é dito convexo se

$$
\Pi\left((1-\theta) f_{a}+\theta f_{b}\right) \leq(1-\theta) \Pi\left(f_{a}\right)+\theta \Pi\left(f_{b}\right), \quad \forall f_{a}, f_{b} \in B, \quad \forall \theta \in[0,1]
$$

Um funcional $\Pi: B \mapsto \mathbf{R}$ é dito estritamente convexo se

$$
\Pi\left((1-\theta) f_{a}+\theta f_{b}\right)<(1-\theta) \Pi\left(f_{a}\right)+\theta \Pi\left(f_{b}\right), \quad \forall f_{a}, f_{b} \in B, \quad \forall \theta \in(0,1)
$$

Considere $V_{h}\left(f_{0}\right)=\left\{\Omega \subset B \mid \forall f \in \Omega, d\left(f, f_{0}\right)<\varepsilon\right\}$ uma vizinhança de $f_{0}$.

\section{Definição 2.51}

Diz-se que o funcional $\Pi: B \mapsto \mathbf{R}$ passa por um mínimo local em $f_{0}$ se existir uma vizinhança de $f_{0}$ na qual

$$
\Pi(f) \geq \Pi\left(f_{0}\right), \quad \forall f \in V_{h}\left(f_{0}\right) .
$$

\section{Definição 2.52}

Diz-se que este mínimo é global se

$$
\Pi(f) \geq \Pi\left(f_{0}\right), \quad \forall f \in B
$$

Diz-se que este mínimo é estrito se

$$
\Pi(f)>\Pi\left(f_{0}\right), \quad \forall f \in V_{h}\left(f_{0}\right) \mid d\left(f, f_{0}\right) \neq 0 .
$$

\section{Definição 2.53}


Diz-se que o funcional $\Pi: B \mapsto \mathbf{R}$ passa por um máximo local em $f_{0}$ se existir uma vizinhança de $f_{0}$ na qual

$$
\Pi(f) \leq \Pi\left(f_{0}\right), \quad \forall f \in V_{h}\left(f_{0}\right) .
$$

\section{Definição 2.54}

Diz-se que este máximo é global se

$$
\Pi(f) \leq \Pi\left(f_{0}\right), \quad \forall f \in B
$$

Diz-se que este máximo é estrito se

$$
\Pi(f)<\Pi\left(f_{0}\right), \quad \forall f \in V_{h}\left(f_{0}\right) \mid d\left(f, f_{0}\right) \neq 0 .
$$

\section{Observações:}

2.3 Funcionais convexos possuem pelo menos um mínimo global. Quando eles são estritamente convexos este mínimo não só existe mas é único.

2.4 Diz-se também que $\Pi\left(f_{0}\right)$ é um extremo de $\Pi$ e que $f_{0}$ é um extremante.

\subsection{EQUAÇÕES DIFERENCIAIS ORDINÁRIAS}

Uma equação diferencial ordinária é uma equação na qual a incógnita é uma função de uma variável e nesta equação comparecem também as derivadas da função incógnita até ordem $k$. Diz-se então que a equação diferencial é de ordem $k$. Pode-se tornar esta definição mais precisa.

\section{Definição 2.55}

Seja $\boldsymbol{P}$ um aberto de $\mathbf{R}^{k+2}$ e seja uma função $F: P \mapsto \mathbf{R}$. A equação

$$
F\left(x, u, u^{\prime}, u^{\prime \prime}, \ldots, u^{k}\right)=0
$$

é uma equação diferencial ordinária (EDO) de ordem $k$.

\section{Definição 2.56}


Uma solução desta equação é uma função $v:(a, b) \mapsto \mathbf{R}$, onde $(a, b) \subset$ $\mathbf{R}$ é um intervalo aberto de $\mathbf{R}$, tal que:

a) $v, v^{\prime}, v^{\prime \prime}, \ldots, v^{k}$ são contínuas em $(a, b)$;

b) $\left\{x, v(x), v^{\prime}(x), v^{\prime \prime}(x), \ldots, v^{k}(x)\right\} \in \boldsymbol{P} \forall x \in(a, b)$;

c) $F\left(x, v, v^{\prime}, v^{\prime \prime}, \ldots, v^{k}\right)=0$.

\section{Definição 2.57}

Uma EDO é dita normal se puder ser colocada na seguinte forma

$$
u^{k}=f\left(x, u, u^{\prime}, \ldots, u^{k-1}\right) \text {. }
$$

Neste trabalho aparecem apenas EDO's normais e somente estas serão consideradas neste texto.

Definição 2.58

Uma EDO $F\left(x, u, u^{\prime}, u^{\prime \prime}, \ldots, u^{k}\right)=0$ é dita linear se tiver a seguinte forma

$$
u^{k}+a_{1}(x) u^{k-1}+a_{2}(x) u^{k-2}+\ldots+a_{k-1}(x) u^{\prime}+a_{k}(x) u=b(x) .
$$

\section{Definição 2.59}

Uma EDO linear é dita homogênea se $b(x)=0$.

Pode-se generalizar as definições acima ao se admitir que $u$ seja um vetor $\boldsymbol{u}(x)$ com $n$ componentes $u_{1}(x), u_{2}(x), \ldots, u_{n}(x)$. No lugar de $F$ ter-se-á uma função vetorial $\boldsymbol{F}$ de $n$ componentes e $\boldsymbol{F}\left(x, \boldsymbol{u}, \boldsymbol{u}^{\prime}, \boldsymbol{u}^{\prime \prime}, \ldots, \boldsymbol{u}^{k}\right)=\mathbf{0}$ descreve um sistema de $n$ equações diferenciais ordinárias de ordem $k$. A definição de um sistema de equações diferenciais de ordem $k$ é a seguinte:

\section{Definição 2.60}

Seja $\boldsymbol{P}$ um aberto de $\mathbf{R}^{n(k+1)+1}$ e seja uma função $\boldsymbol{F}: \boldsymbol{P} \mapsto \mathbf{R}^{n}$. A equação

$$
\boldsymbol{F}\left(x, \boldsymbol{u}, \boldsymbol{u}^{\prime}, \boldsymbol{u}^{\prime \prime}, \ldots, \boldsymbol{u}^{k}\right)=\mathbf{0}
$$

é um sistema de equações diferenciais ordinárias (SEDO) de ordem $k$.

\section{Definição 2.61}


Uma solução desta equação é uma função $v:(a, b) \mapsto \mathbf{R}^{n}$, onde $(a, b) \subset$ $\mathbf{R}$ é um intervalo aberto de $\mathbf{R}$, tal que

a) $\boldsymbol{v}, \boldsymbol{v}^{\prime}, \boldsymbol{v}^{\prime \prime}, \ldots, \boldsymbol{v}^{k}$ são contínuas em $(a, b)$

b) $\left\{x, \boldsymbol{v}(x), \boldsymbol{v}^{\prime}(x), \boldsymbol{v}^{\prime \prime}(x), \ldots, \boldsymbol{v}^{k}(x)\right\} \in \boldsymbol{P} \forall x \in(a, b)$

c) $\boldsymbol{F}\left(x, \boldsymbol{v}, \boldsymbol{v}^{\prime}, \boldsymbol{v}^{\prime \prime}, \ldots, \boldsymbol{v}^{k}\right)=\mathbf{0}$

Fato 2.3:

O conjunto das soluções da equação diferencial ordinária linear homogênea de ordem $k$

$$
a_{k}(x) y^{(k)}+a_{k-1}(x) y^{(k-1)}+\ldots+a_{2}(x) y^{\prime \prime}+a_{1}(x) y^{\prime}+a_{0}(x) y=0
$$

é um espaço vetorial.

Definição 2.62

Um SEDO é dito normal se puder ser colocado na seguinte forma

$$
\boldsymbol{u}^{k}=\boldsymbol{f}\left(x, \boldsymbol{u}, \boldsymbol{u}^{\prime}, \ldots, \boldsymbol{u}^{k-1}\right)
$$

Restringir-se-á esta apresentação à SEDO's normais.

\section{Definição 2.63}

Um SEDO $\boldsymbol{F}\left(x, \boldsymbol{u}, \boldsymbol{u}^{\prime}, \boldsymbol{u}^{\prime \prime}, \ldots, \boldsymbol{u}^{k}\right)=\mathbf{0}$ é dito linear se tiver a seguinte forma

$$
\boldsymbol{u}^{k}+\boldsymbol{A}_{1}(x) \boldsymbol{u}^{k-1}+\boldsymbol{A}_{2}(x) \boldsymbol{u}^{k-2}+\ldots+\boldsymbol{A}_{k-1}(x) \boldsymbol{u}^{\prime}+\boldsymbol{A}_{k}(x) \boldsymbol{u}=\boldsymbol{b}(x)
$$

onde $\boldsymbol{A}_{i}(x)$ são matrizes quadradas de ordem $n$, cujos elementos são funções de $x$.

\section{Definição 2.64}

Um SEDO é dito homogêneo se $\boldsymbol{b}(x)=\mathbf{0}$.

Seja um SEDO de primeira ordem dado por

$$
\boldsymbol{u}^{\prime}=\boldsymbol{f}(x, \boldsymbol{u})
$$


Resolver um problema de valor inicial (PVI) de primeira ordem significa encontrar a solução $\boldsymbol{v}(x)$ de (2.35) que satisfaça a condição inicial

$$
\boldsymbol{v}\left(x_{0}\right)=v_{0},
$$

onde $\boldsymbol{v}_{0}$ é um vetor constante.

Considere agora um SEDO de segunda ordem dado por

$$
\boldsymbol{u}^{\prime \prime}=\boldsymbol{f}\left(x, \boldsymbol{u}, \boldsymbol{u}^{\prime}\right)
$$

\section{Definição 2.66}

Resolver um problema de valor inicial (PVI) de segunda ordem significa encontrar a solução $\boldsymbol{v}(x)$ de (2.37) que satisfaça as condições iniciais

$$
\begin{aligned}
& \boldsymbol{v}\left(x_{0}\right)=\boldsymbol{v}_{0} \mathrm{e} \\
& \boldsymbol{v}^{\prime}\left(x_{0}\right)=\boldsymbol{v}^{\prime}{ }_{0} \\
& \text { ou } \\
& \boldsymbol{v}\left(x_{k}\right)=\boldsymbol{v}_{k} \mathrm{e} \\
& \boldsymbol{v}\left(x_{i}\right)=\boldsymbol{v}_{i}, k \neq i .
\end{aligned}
$$

onde $\boldsymbol{v}_{0}, \boldsymbol{v}_{0}^{\prime}, \boldsymbol{v}_{k}$ e $\boldsymbol{v}_{k}^{\prime}$ são vetores constantes.

Teorema 2.1:

Seja um SEDO de segunda ordem na forma normal

$$
\boldsymbol{u}^{\prime \prime}=\boldsymbol{f}\left(x, \boldsymbol{u}, \boldsymbol{u}^{\prime}\right)
$$

definido em $\boldsymbol{P} \subset \mathbf{R}^{2 n+1}$, onde $n$ é a dimensão de $\boldsymbol{u}$. Se as componentes $f_{i}$ do vetor $\boldsymbol{f}: \boldsymbol{P} \rightarrow \mathbf{R}^{n}$ forem contínuas com derivadas contínuas em $\boldsymbol{P}$, então existe uma única solução $\boldsymbol{v}$ de (2.39) que satisfaz as condições iniciais (2.38).

\subsection{PROGRAMAÇÃO MATEMÁTICA}




\subsubsection{OTIMIZAÇÃO SEM RESTRIÇÕES}

Considere-se o problema de minimizar uma função $f(\boldsymbol{x}), \boldsymbol{x} \in \mathbf{R}^{n}$. O problema pode ser colocado na forma

$$
\begin{gathered}
\text { minimizar } f(\boldsymbol{x}) \\
\boldsymbol{x} \in \mathbf{R}^{n} .
\end{gathered}
$$

Admite-se que a função $f(\boldsymbol{x}) \in C_{2}$. A função $f(\boldsymbol{x})$ é denominada função objetivo.

Nesta seção, serão estabelecidas condições que devem ser satisfeitas por um ponto, para que seja um mínimo local do problema (2.40). Também serão descritas as propriedades de convexidade da função objetivo que asseguram que o ponto encontrado seja um ponto de mínimo global.

\section{Teorema 2.2:}

Para que um ponto $\boldsymbol{x}^{*}$ seja um ponto de mínimo local do problema (2.40) é suficiente que o gradiente da função objetivo em $\boldsymbol{x}^{*}$ seja nulo, ou seja, $\nabla f\left(\boldsymbol{x}^{*}\right)=\mathbf{0}$ e que a matriz Hessiana $\nabla^{2} f\left(\boldsymbol{x}^{*}\right)$ seja definida positiva, isto é

$$
\left\langle\boldsymbol{d}, \nabla^{2} f\left(\boldsymbol{x}^{*}\right) \boldsymbol{d}\right\rangle>0 \quad \forall \boldsymbol{d} \neq 0
$$

Teorema 2.3:

Seja $f(\boldsymbol{x})$ uma função convexa definida em $\mathbf{R}^{n}$ e seja $\Omega$ o conjunto dos pontos $\boldsymbol{x} \in \mathbf{R}^{n}$ onde $f(\boldsymbol{x})$ atinge seu mínimo. Então $\Omega$ é convexo e todo mínimo local é um ponto de mínimo global.

Teorema 2.4:

Seja $f(\boldsymbol{x}) \in C_{1}$ uma função convexa. Se exister um $\boldsymbol{x}^{*} \in \mathbf{R}^{n}$ tal que para todo $\boldsymbol{y} \in \mathbf{R}^{n}$,

$$
\left\langle\nabla f\left(\boldsymbol{x}^{*}\right),\left(\boldsymbol{y}-\boldsymbol{x}^{*}\right)\right\rangle \geq 0
$$

então $\boldsymbol{x}^{*}$ é um ponto de mínimo global de $f(\boldsymbol{x})$.

\subsubsection{OTIMIZAÇÃO COM RESTRIÇÕES}

Considere-se o problema geral de otimização da forma 


$$
\begin{array}{lrl}
\text { minimizar } & f(\boldsymbol{x}), \boldsymbol{x} \in \mathbf{R}^{n}, & \\
& \\
\text { sujeito a } & g_{i}(\boldsymbol{x})=0, \quad i \in E \\
& g_{i}(\boldsymbol{x}) \leq 0, \quad i \in I
\end{array}
$$

onde a função $f(\boldsymbol{x})$ é denominada função objetivo e as funções $g_{i}(\boldsymbol{x}), i=1, \ldots, m$ são denominadas restrições. $E$ é o conjunto dos índices das restrições de igualdade e $I$ é o conjunto dos índices das restrições de desigualdade. A solução de (2.41) é denominada de solução ou ponto ótimo e será denotada por $\boldsymbol{x}^{*}$.

Quando um ponto $\boldsymbol{x} \in \mathbf{R}^{n}$ satisfaz todas as restrições, diz-se que ele é viável, e o conjunto de todos os pontos viáveis é denominado de região viável $\Gamma$.

Admite-se que as funções $f(\boldsymbol{x})$ e $g_{i}(\boldsymbol{x}) \in C_{2}\left[\mathbf{R}^{n}\right]$.

\section{Definição 2.67}

As restrições $\left\{g_{i}(\boldsymbol{x}), i \in I \mid g_{i}(\boldsymbol{x})=0\right\}$ são denominadas restrições ativas em $\boldsymbol{x}$. Indicar-se-á por $I^{*}$ o conjunto dos índices referentes às restrições ativas em $\boldsymbol{x}$.

\section{Definição 2.68}

As restrições $\left\{g_{i}(\boldsymbol{x}) \mid\left\|g_{i}(\boldsymbol{x})\right\| \leq \varepsilon\right\}$ são denominadas restrições $\varepsilon$-ativas em $\boldsymbol{x}$.

\section{Definição 2.69}

Diz-se que um ponto $\boldsymbol{x}_{v}$, que satisfaz as restrições $g_{i}(\boldsymbol{x}), i \in E \cup I^{*}$, é um ponto regular se os vetores gradientes $\left\{\nabla g_{i}\left(\boldsymbol{x}_{v}\right), i \in E \cup I^{*}\right\}$ forem linearmente independentes.

Denomina-se a função Lagrangeana a função definida por:

$$
L(\boldsymbol{x}, \boldsymbol{u})=f(\boldsymbol{x})+\sum_{i=1}^{m} u_{i} g_{i}(\boldsymbol{x})
$$

onde $u_{i} \in \mathbf{R}, i=1, \ldots, m$ são os multiplicadores de Lagrange.

Indica-se por $\nabla L(\boldsymbol{x}, \boldsymbol{u})=\left[\begin{array}{l}\nabla_{x} L(\boldsymbol{x}, \boldsymbol{u}) \\ \nabla_{u} L(\boldsymbol{x}, \boldsymbol{u})\end{array}\right]$ o vetor gradiente de $L(\boldsymbol{x}, \boldsymbol{u})$, onde $\nabla_{x}$ indica as derivadas parciais em relação à $x_{i}, i=1, \ldots, n$, e $\nabla_{u}$ indica as derivadas em relação à $u_{j}, j=1, \ldots, m$ 
O vetor gradiente da função Lagrangeana em relação à $\boldsymbol{x}$ em $\left(\boldsymbol{x}^{*}, \boldsymbol{u}^{*}\right) \in \boldsymbol{R}^{n+m}$ é dado por:

$$
\nabla_{x} L\left(\boldsymbol{x}^{*}, \boldsymbol{u}^{*}\right)=\nabla f\left(\boldsymbol{x}^{*}\right)+\sum_{i=1}^{m} u_{i}^{*} \nabla g_{i}\left(\boldsymbol{x}^{*}\right)
$$

onde $\boldsymbol{u}^{*}$ é o vetor dos multiplicadores de Lagrange no ponto ótimo.

Analogamente ao vetor gradiente, a matriz Hessiana da função Lagrangeana em relação à $\boldsymbol{x}$ no ponto $\left(\boldsymbol{x}^{*}, \boldsymbol{u}^{*}\right) \in \boldsymbol{R}^{n+m}$ é dada por:

$$
\nabla_{x}^{2} L\left(\boldsymbol{x}^{*}, \boldsymbol{u}^{*}\right)=\nabla^{2} f\left(\boldsymbol{x}^{*}\right)+\sum_{i=1}^{m} u_{i} \nabla^{2} g_{i}\left(\boldsymbol{x}^{*}\right)
$$

Teorema 2.5 (Kuhn-Tucker):

Seja $\boldsymbol{x}^{*}$ um ponto de mínimo local do problema (2.42). Se $\boldsymbol{x}^{*}$ é um ponto regular, então existem multiplicadores de Lagrange $\boldsymbol{u}^{*}=$ $\left[u_{1}^{*}, u_{2} *, \ldots, u_{m} *\right]^{T}$, de tal forma que $\boldsymbol{x}^{*}$ e $\boldsymbol{u}^{*}$ satisfazem o seguinte sistema de equações:

$$
\begin{array}{ll}
\nabla f\left(\boldsymbol{x}^{*}\right)+\sum_{i=1}^{m} u_{i} * \nabla g_{i}\left(\boldsymbol{x}^{*}\right)=\mathbf{0} \\
g_{i}\left(\boldsymbol{x}^{*}\right)=0, & i \in E \\
g_{i}\left(\boldsymbol{x}^{*}\right) \leq 0, & i \in I \\
u_{i}^{*}>0, & i \in I \\
u_{i} * g_{i}\left(\boldsymbol{x}^{*}\right)=0 . & \forall i
\end{array}
$$

As equações acima são denominadas Condições de Kuhn-Tucker.

\section{Teorema 2.6:}

Para que o ponto $\boldsymbol{x}^{*}$ seja um ponto de mínimo local do problema (2.42) é necessário que seja um ponto regular, que satisfaça as condições de KuhnTucker e ainda que

$$
\left\langle\boldsymbol{d}^{T}, \nabla_{x}^{2} L\left(\boldsymbol{x}^{*}, \boldsymbol{u}^{*}\right) \boldsymbol{d}\right\rangle \geq 0 \quad \forall \boldsymbol{d} \in \boldsymbol{G}^{*} .
$$

onde $G^{*}$ é dado por: 


$$
\begin{gathered}
\boldsymbol{G}^{*}=\left\{\boldsymbol{d} / \boldsymbol{d} \neq \mathbf{0}, \boldsymbol{d}^{T} \nabla g_{i}\left(\boldsymbol{x}^{*}\right)=0, i \in\left(E \cup I^{*}\right) / u_{i}^{*}>0\right. \\
\left.\mathrm{e} \boldsymbol{d}^{T} \nabla g_{i}\left(x^{*}\right) \leq 0, i \in I^{*} / u_{i}^{*}=0\right\}
\end{gathered} .
$$

Considere-se o problema:

$$
\begin{aligned}
& \begin{array}{l}
\text { minimizar } \quad f(\boldsymbol{x}) \\
\text { sujeito às restrições } \\
\qquad g_{i}(\boldsymbol{x}) \leq 0, \quad i=1, . . l \\
g_{i}(\boldsymbol{x})=0, \quad i=l+1, \ldots, m
\end{array} \\
& \boldsymbol{x} \in \mathbf{R}^{n} .
\end{aligned}
$$

Se a função objetivo e as restrições forem convexas o problema é dito de programação convexa (Medrano [14]). Para problema de programação convexa valem os seguintes resultados:

\section{Teorema 2.7:}

Toda solução $\boldsymbol{x}^{*}$ de um problema de programação convexa é uma solução global e o conjunto das soluções globais $S$ é um conjunto convexo.

Se no problema de programação convexa a função objetivo for estritamente convexa em $\boldsymbol{R}^{n}$ então toda solução global é única.

\section{Teorema 2.8:}

Se num problema de programação convexa, as funções $f(\boldsymbol{x})$ e $g_{i}(\boldsymbol{x})$ são contínuas com derivadas parciais contínuas até primeira ordem, e se as condições de Kuhn-Tucker estão satisfeitas em $\boldsymbol{x}^{*}$, então o ponto $\boldsymbol{x}^{*}$ é uma solução global do problema de programação convexa. 


\section{Capítulo 3}

\section{PROBLEMA DE OTIMIZAÇÃO COM RESTRIÇÕES DINÂMICAS}

\subsection{INTRODUÇÃO}

Este capítulo apresenta uma revisão do Método dos Multiplicadores que será aplicado no projeto ótimo de sistemas de engenharia. A maneira como serão apresentadas as formulações de problemas de otimização com restrições dinâmicas é mostrada no item 3.2 .

Os problemas de otimização que serão tratados neste texto consistem em determinar variáveis de projeto $\boldsymbol{x}$ que minimizem uma determinada função, denominada função objetivo, sujeito às restrições de igualdade e desigualdade. A idéia básica do método do Lagrangeano aumentado consiste em transformar um problema de otimização com restrições em uma seqüência de problemas sem restrições. A solução dos problemas sem restrições, gera uma seqüência de pontos (soluções ótimas dos problemas sem restrições) que sob certas hipóteses que veremos nos itens 3.3.3 e 3.3.4, convergem para a solução do problema com restrições.

O Algoritmo do Método dos Multiplicadores é descrito no item 3.3.5. Finalmente, a aplicação do Método dos Multiplicadores em problemas com restrições dinâmicas e um Procedimento Computacional implementável são mostrados no item 3.3.6. 


\subsection{FORMULAÇÃO DO PROBLEMA}

Em determinados projetos estruturais e mecânicos, adota-se uma configuração inicial (geométrica e ou física) e verifica a resposta da estrutura ao carregamento aplicado. Se a estrutura não se comportar como desejado, então o projeto é alterado e a estrutura reanalizada. Este processo continua até se obter uma resposta adequada.

O objetivo principal deste trabalho é formular e resolver problemas de otimização estrutural e mecânica que apresentam restrições dinâmicas. Este problema pode ser apresentado na forma:

Problema Pd. Determine $\boldsymbol{x} \in \boldsymbol{R}^{n}$ que minimize a função objetivo $f(\boldsymbol{x})$ sujeito às

Restrições estáticas:

$$
\begin{aligned}
& g_{i}(\boldsymbol{x})=0 ; \quad i=1, l \\
& g_{i}(\boldsymbol{x}) \leq 0 ; \quad i=l+1, m
\end{aligned}
$$

Restrições dinâmicas:

$$
\begin{aligned}
& \text { para todo } t \in\left[t_{\mathrm{o}}, t_{f}\right] \\
& g_{i}(\boldsymbol{x}, t)=g_{i}(\boldsymbol{x}, \boldsymbol{z}(t), \dot{z}(t), \ddot{z}(t), t)=0 ; \quad i=m+1, l^{\prime} \\
& g_{i}(\boldsymbol{x}, t)=g_{i}(\boldsymbol{x}, \boldsymbol{z}(t), \dot{z}(t), \ddot{z}(t), t) \leq 0 ; \quad i=l^{\prime}+1, m^{\prime}
\end{aligned}
$$

No processo de dimensionamento, um conjunto de parâmetros, denominados variáveis de projeto $\boldsymbol{x}$, são selecionados pelo projetista para definir o sistema a ser dimensionado. As variáveis de projeto podem representar momento de inércia, área e rigidez. As equações (3.1) e (3.2) incluem, por exemplo, limites para as variáveis de projeto.

Já as equações (3.3) e (3.4) representam restrições com resposta dinâmica como, por exemplo, valores máximos e mínimos para deslocamentos e tensões. $\boldsymbol{z}(t)$ é o vetor deslocamento que se supõe função contínua de $t$ no intervalo $\left[t_{0}, t\right]$. As funções restrições pertencem ao espaço $C_{2}\left[t_{0}, t_{f}\right]$, onde $t_{0}$ e $t_{f}$ são respectivamente os instantes inicial e final. 
Nos problemas que serão tratados o vetor $\boldsymbol{z}(t)$ deve satisfazer as equações do movimento, representadas pelo sistema de equações diferenciais de segunda ordem:

$$
M(x) \ddot{z}(t)+C(x, \dot{z}(t)) \dot{z}(t)+\boldsymbol{R}(\boldsymbol{x}, \boldsymbol{z}(t))=\boldsymbol{p}(\boldsymbol{x}, t)
$$

com as condições iniciais $\dot{\boldsymbol{z}}\left(t_{0}\right)=\dot{\boldsymbol{z}}_{0}$ e $\boldsymbol{z}\left(t_{0}\right)=\boldsymbol{z}_{0}$, onde $\boldsymbol{M}(\boldsymbol{x})$ e $\boldsymbol{C}(\boldsymbol{x}, \dot{\boldsymbol{z}}(t))$ são respectivamente as matrizes de massa e de amortecimento, o vetor $\boldsymbol{R}(\boldsymbol{x}, \boldsymbol{z}(t))$ é a força restauradora. $\mathrm{O}$ vetor $\boldsymbol{p}(\boldsymbol{x}, t)$ é o vetor das forças generalizado.

Uma vez especificadas as variáveis de projeto $\boldsymbol{x}$, o vetor deslocamento $\boldsymbol{z}(t)$ é determinado resolvendo-se a equação (3.5). $\boldsymbol{z}$ representa a resposta da estrutura ao carregamento aplicado, por isso suas componentes são denominadas variáveis de estado, pois através delas determina-se a configuração da estrutura em qualquer instante $t \in\left[t_{0}, t_{f}\right]$. As equações (3.5) são denominadas de equações de estado.

\subsection{MÉTODO DO LAGRANGEANO AUMENTADO}

\subsubsection{INTRODUÇÃO}

Nesta seção é descrito o método dos multiplicadores que será utilizado para resolver problemas de otimização com restrições dinâmicas.

O método dos multiplicadores, segundo Arora, Chahande e Paeng [11], foi proposto inicialmente por Haarhoff e Powell, e a seguir por Buys. Este método está largamente descrito na literatura, como por exemplo em Fletcher [25], Polak [8], Chahande e Arora [1] e outros. Nesta seção realiza-se um resumo sucinto deste método para o caso estático baseado nestes textos.

Considera-se então o seguinte problema de otimização, com restrições de igualdade e desigualdade:

Problema P. Determine $\boldsymbol{x} \in \boldsymbol{R}^{n}$ que minimize a função objetivo $f(\boldsymbol{x})$ sujeito a

restrições de igualdade:

restrições de desigualdade:

$$
g_{i}(\boldsymbol{x})=0 ; \quad i=1, l
$$




$$
L(\boldsymbol{x}, \boldsymbol{u})=f(\boldsymbol{x})+\sum_{i=1}^{m} u_{i} g_{i}(\boldsymbol{x})
$$

onde $\boldsymbol{u} \in \boldsymbol{R}^{m}$ é o vetor dos multiplicadores de Lagrange. O método dos multiplicadores introduz termos de penalidade relacionados com cada restrição. Os multiplicadores de Lagrange e os parâmetros de penalidade, relacionados a cada restrição, são usados na construção de um Lagrangeano aumentado que pode ser escrito como

$$
\Phi(\boldsymbol{x}, \boldsymbol{u}, \boldsymbol{r})=f(\boldsymbol{x})+P(\boldsymbol{g}(\boldsymbol{x}), \boldsymbol{u}, \boldsymbol{r})
$$

onde $P(\boldsymbol{g}(\boldsymbol{x}), \boldsymbol{u}, \boldsymbol{r})$ é uma função de penalidade e $\boldsymbol{r} \in \boldsymbol{R}^{m}$ contém parâmetros de penalidade. Será visto mais tarde que $\Phi(\boldsymbol{x}, \boldsymbol{u}, \boldsymbol{r})$ pode ser formado adicionando termos de penalidade ao Lagrangeano em (3.8). De agora em diante $\Phi(\boldsymbol{x}, \boldsymbol{u}, \boldsymbol{r})$ será denominado de Lagrangeano aumentado.

O método pode ser resumido pelo seguinte algoritmo:

\section{Algoritmo I:}

Passo 1. Faça $k=0$. Adote multiplicadores de lagrange $\boldsymbol{u}$ e parâmetros de penalidades $\boldsymbol{r}$.

Passo 2. Minimize $\Phi\left(\boldsymbol{x}, \boldsymbol{u}^{k}, \boldsymbol{r}^{k}\right)$ em relação a $\boldsymbol{x}$. Considere $\boldsymbol{x}^{k}$ a solução.

Passo 3. Se os critérios de convergência forem satisfeitos, pare o processo de iteração.

Passo 4. Atualize $\boldsymbol{u}^{k}$, e $\boldsymbol{r}^{k}$ se necessário.

Passo 5. Faça $k=k+1$ e vá ao passo 2 .

Conceitualmente o método dos multiplicadores é muito simples e sua essência está contida nos passos 2 e 4 . O desempenho do método depende fortemente de como estes passos são executados. A precisão requerida para o mínimo de $\Phi$ no algoritmo de minimização sem restrições utilizado no passo 2 influencia o comportamento e a eficiência do método. Estes dois aspectos são essenciais para o bom desempenho do método e serão discutidos posteriormente.

Como no decorrer deste trabalho somente serão efetuados derivadas em relação à $\boldsymbol{x}$, indicar-se-á $\nabla_{x}=\nabla$.

No passo 2 o algoritmo de minimização sem restrições requer a adoção de um critério de parada. Neste trabalho considerar-se-á os seguintes critérios sugeridos em $[\mathbf{1}, \mathbf{1 1}]$ : 
i. $\quad k<p \quad$,

onde $p$ é o número máximo de iterações,

ii. $\quad\left\|\nabla \Phi\left(\boldsymbol{x}^{k}, \boldsymbol{u}^{k}, \boldsymbol{r}\right)\right\| \leq \varepsilon$

$\mathrm{e}$

$$
\text { iii. } \quad K_{b}=\max \left\{\max _{1 \leq i \leq l}\left|g_{i}\right| ; \max _{l+1 \leq i \leq m}\left|\max \left(g_{i},-\theta_{i}\right)\right|\right\} \text {, }
$$

onde $\theta_{i}=u_{i} / r_{i}$.

Em (3.11) $\varepsilon$ é a tolerância estabelecida e em (3.12) $K_{b}$ representa a máxima violação de restrição. Caso o algoritmo utilizado neste passo não convirja, a condição (3.10) impõe um número máximo de iterações. Arora, Chahande e Paeng [11] observaram em diversos exemplos analisados que o valor ideal para $p$ é igual a $2 n$.

Um outro ponto a ser estudado diz respeito ao passo 4 do algoritmo, ou seja, a atualização dos multiplicadores de Lagrange. $\mathrm{O}$ procedimento para acréscimo dos multiplicadores, passo 4, para restrições de igualdade, é feito na forma

$$
\theta_{i}^{k+1}=\theta_{i}^{k}+g_{i}\left(\boldsymbol{x}^{k}\right) ; i=1, \ldots, l
$$

sendo que $\theta_{i}^{k}$ define o multiplicador de Lagrange por $u_{i}^{k}=r_{i} \theta_{i}^{k}$ na $k$-ésima iteração do algoritmo geral. Observe que (3.13) não requer o cálculo do gradiente das restrições individualmente.

Quando restrições de desigualdade estão presente, os multiplicadores podem ser acrescidos usando os valores das funções violação de restrição como

$$
\theta_{i}^{k+1}=\theta_{i}^{k}+\max \left(g_{i}\left(\boldsymbol{x}^{k}\right),-\theta_{i}^{k}\right) ; \quad \theta_{i}^{k+1} \geq 0 ; \quad i=l+1, m .
$$

Estas expressões são denominadas fórmulas de Hestenes-Powell. Existem outras maneiras de se acrescer os multiplicadores, ver Luemberger [5].

Nas seções seguintes detalharemos alguns conceitos fundamentais e procedimentos associados ao método dos Multiplicadores.

\subsubsection{LAGRANGEANO AUMENTADO}


O Lagrangeano aumentado pode ser definido de diversas maneiras. Uma delas é o de Rockafellar que inclui termos de penalidade quadrática para o Problema P (Arora, Chahande e Paeng [11]):

$$
\bar{\Phi}(\boldsymbol{x}, \theta, \boldsymbol{r})=f(\boldsymbol{x})+\frac{1}{2} \sum_{i=1}^{l} r_{i}\left[\left(g_{i}+\theta_{i}\right)^{2}-\theta_{i}^{2}\right]+\frac{1}{2} \sum_{i=l+1}^{m} r_{i}\left[\left(g_{i}+\theta_{i}\right)_{+}^{2}-\theta_{i}^{2}\right]
$$

O símbolo $(h)_{+}$significa $\max (0, h)$. Outra definição usual do Lagrangeano e que será adotado neste trabalho é dado por Fletcher [23]:

$$
\Phi(\boldsymbol{x}, \theta, \boldsymbol{r})=f(\boldsymbol{x})+\frac{1}{2} \sum_{i=1}^{l} r_{i}\left(g_{i}+\theta_{i}\right)^{2}+\frac{1}{2} \sum_{i=l+1}^{m} r_{i}\left(g_{i}+\theta_{i}\right)_{+}^{2}
$$

A relação entre os dois funcionais é dada por

$$
\bar{\Phi}(\boldsymbol{x}, \boldsymbol{\theta}, \boldsymbol{r})=\Phi(\boldsymbol{x}, \boldsymbol{\theta}, \boldsymbol{r})-\frac{1}{2} \sum_{i=1}^{m} r_{i} \theta_{i}^{2}
$$

O segundo termo do membro direito não depende de $\boldsymbol{x}$. Logo a solução $\boldsymbol{x} *$, de ambos os problemas, dados $\boldsymbol{r}$ e $\theta$, é a mesma. Porém, os funcionais têm valores diferente.

O Lagrangeano aumentado em função de $\boldsymbol{u}$ é:

$$
\bar{\Phi}(\boldsymbol{x}, \boldsymbol{u}, \boldsymbol{r})=\left\{\begin{array}{l}
f(\boldsymbol{x})+\sum_{i=1}^{l}\left(\frac{1}{2} r_{i} g_{i}^{2}+u_{i} g_{i}\right)+\sum_{i=l+1}^{m}\left(\frac{1}{2} r_{i} g_{i}^{2}+u_{i} g_{i}\right) ; \operatorname{se}\left(g_{\mathrm{i}}+\frac{u_{i}}{r_{i}}\right) \geq 0 \text { para } i>l \\
f(\boldsymbol{x})+\sum_{i=1}^{l}\left(\frac{1}{2} r_{i} g_{i}^{2}+u_{i} g_{i}\right)-\sum_{i=l+1}^{m}\left(\frac{u_{i}^{2}}{2 r_{i}}\right) ; \operatorname{se}\left(g_{\mathrm{i}}+\frac{u_{i}}{r_{i}}\right)<0 \text { para } i>l
\end{array}\right.
$$

Observa-se claramente que $\bar{\Phi}(\boldsymbol{x}, \boldsymbol{u}, \boldsymbol{r})$ contém termos de penalidade quadrática $1 / 2 r_{i} g_{i}^{2}$, $i=1, \ldots, l$, adicionados ao funcional definido em (3.8). Por isso (3.18) é denominado de Lagrangeano Aumentado.

A forma quadrática do Lagrangeano aumentado, dada em (3.16), tem sido amplamente usado em implementações práticas do método dos multiplicadores. Às vezes pode ser vantajoso usar outras formas do método [11].

\subsubsection{CONDIÇÕES NECESSÁRIAS E SUFICIENTES}


Nesta seção estudar-se-á a relação entre as condições necessárias e suficientes que deve satisfazer a solução do Problema $\mathrm{P}$ e a sequência de soluções ótimas do problema de minimização do funcional de Fletcher (3.16) quando $k \rightarrow \infty$.

Considera-se que $f(\boldsymbol{x})$ e $g_{i}(\boldsymbol{x}) \in C_{2}$ e que os vetores $\nabla g_{i}\left(\boldsymbol{x}^{*}\right), i \in E \cup I^{*}$, sejam linearmente independentes, onde $E$ é o conjunto dos índices para as restrições de igualdade definido como $E=\{i: i=1, l\}$ e $I$ é o conjunto dos índices para restrições de desigualdade definido como $I=\left\{i: u_{i}>0, i=l+1, m\right\}$. O conjunto dos índices referentes às restrições ativas, conforme definição 2.61 , é denotado por $I^{*}$.

Seja $\boldsymbol{x}^{*}$ a solução do problema $\mathrm{P}$ e $\boldsymbol{u}^{*}$ o correspondente vetor dos multiplicadores de Lagrange no ponto ótimo.

Segundo o Teorema 2.4, para o problema $\mathrm{P}$ existem únicos multiplicadores de Lagrange $\boldsymbol{u}^{*}$, de tal forma que $\boldsymbol{x}^{*}$ e $\boldsymbol{u}^{*}$ satisfazem

$$
\nabla f\left(\boldsymbol{x}^{*}\right)+\sum_{i=1}^{m} u_{i}^{*} \nabla g_{i}\left(\boldsymbol{x}^{*}\right)=\mathbf{0}
$$

Por outro lado, seja $\boldsymbol{x}^{k}$ o mínimo de $\Phi\left(\boldsymbol{x}, \boldsymbol{u}^{k}, \boldsymbol{r}\right)$, onde $\boldsymbol{r}>\boldsymbol{0}$ e $\boldsymbol{u}^{k}$ são os valores correntes dos parâmetros na $k$-ésima iteração do método dos multiplicadores. A condição para que $\boldsymbol{x}^{k}$ seja o mínimo do Lagrangeano Aumentado é $\nabla \Phi\left(\boldsymbol{x}^{k}, \theta^{k}, \boldsymbol{r}\right)=\mathbf{0}$. Utilizando o fato de que

$$
\nabla\left(g_{i}\left(\boldsymbol{x}^{k}\right)+\theta_{i}^{k}\right)_{+}^{2}=2\left(g_{i}\left(\boldsymbol{x}^{k}\right)+\theta_{i}^{k}\right)_{+} \nabla g_{i}\left(\boldsymbol{x}^{k}\right) ; \quad i=l+1, m
$$

demonstrado por Luemberger [5], tem-se

$$
\begin{aligned}
\nabla \Phi\left(\boldsymbol{x}^{k}, \theta^{k}, \boldsymbol{r}\right)= & \nabla f\left(\boldsymbol{x}^{k}\right)+\sum_{i=1}^{l} r_{i}\left(g_{i}\left(\boldsymbol{x}^{k}\right)+\theta_{i}^{k}\right) \nabla g_{i}\left(\boldsymbol{x}^{k}\right) \\
& +\sum_{i=l+1}^{m} r_{i}\left(g_{i}\left(\boldsymbol{x}^{k}\right)+\theta_{i}^{k}\right)_{+} \nabla g_{i}\left(\boldsymbol{x}^{k}\right)=\mathbf{0}
\end{aligned}
$$

Observa-se que $\left(g_{i}+\theta_{i}\right)_{+}=\max \left(\mathrm{g}_{\mathrm{i}}+\theta_{i}, 0\right)=\theta_{i}+\max \left(-\theta_{i}, \mathrm{~g}_{\mathrm{i}}\right)$, e portanto (3.20) pode ser escrita na forma 


$$
\begin{aligned}
\nabla \Phi\left(\boldsymbol{x}^{k}, \boldsymbol{\theta}^{k}, \boldsymbol{r}\right)= & \nabla f\left(\boldsymbol{x}^{k}\right)+\sum_{i=1}^{l} r_{i}\left(\theta_{i}^{k}+g_{i}\left(\boldsymbol{x}^{k}\right)\right) \nabla g_{i}\left(\boldsymbol{x}^{k}\right) \\
& +\sum_{i=l+1}^{m} r_{i}\left[\theta_{i}^{k}+\max \left(-\theta_{i}, g_{i}\left(\boldsymbol{x}^{k}\right)\right)\right] \nabla g_{i}\left(\boldsymbol{x}^{k}\right)=\mathbf{0}
\end{aligned}
$$

Arora, Chahande e Paeng [11] demonstram que $\lim _{k \rightarrow \infty} \theta_{i}^{k}=\theta_{i}^{*}(i=1, m)$ e portanto

$$
\begin{gathered}
g_{i}\left(\boldsymbol{x}^{k}\right)=0 ; \quad i=1, l \\
\max \left(-\theta_{i}, g_{i}\left(\boldsymbol{x}^{k}\right)\right)=0 ; \quad i=l+1, m
\end{gathered}
$$

Substituindo-se (3.22) e (3.23) em (3.21), obtém-se (3.24). Por comparação, pode-se concluir que $\boldsymbol{x}^{k}=\boldsymbol{x}^{*}$ e $r_{i} \theta_{i}^{k}=r_{i} \theta_{i}^{*}=u_{i}^{*}$. Observe que (3.23) pode ser satisfeita somente quando $\theta_{i}^{k}=0$ ou $g_{i}\left(\boldsymbol{x}_{k}\right)=0$ ou ambos nulos. Estas igualdades podem ser escritas como $\theta_{i}^{k} \mathrm{~g}_{i}\left(\boldsymbol{x}_{k}\right)=0 ; i=l+1, m$. Assim as equações (3.21) à (3.23) são equivalentes às condições de Kuhn-Tucker que são as condições necessárias para o Problema P:

$$
\begin{gathered}
\nabla f\left(\boldsymbol{x}^{k}\right)+\sum_{i=1}^{m} u_{i}^{k} \nabla g_{i}\left(\boldsymbol{x}^{k}\right)=\mathbf{0} \\
g_{i}\left(\boldsymbol{x}^{k}\right)=0 ; \quad i=1, l \\
u_{i}^{k} \geq 0, \mathrm{~g}_{i}\left(\boldsymbol{x}^{k}\right) \leq 0 \text { e } u_{i}^{k} \mathrm{~g}_{i}\left(\boldsymbol{x}^{k}\right)=0 ; i=l+1, m .
\end{gathered}
$$

Da discussão anterior é evidente que o objetivo é escolher $u_{i}$ (ou $\theta_{i}$ ) em (3.13) e (3.14) tal que $g_{i}\left(\boldsymbol{x}^{k}\right) \rightarrow 0 ; i=1, l$ e $\max \left(-\theta_{i}^{k}, g_{i}\left(\boldsymbol{x}^{k}\right)\right) \rightarrow 0 ; i=l+1, m$. Isto resulta que $\boldsymbol{x}^{k} \rightarrow \boldsymbol{x}^{*}$.

Prova-se [11] que $\boldsymbol{x}^{*}$ é um mínimo local isolado de $\Phi\left(\boldsymbol{x}, \boldsymbol{u}^{*}, \boldsymbol{r}\right)$ considerando-se que $r_{i}$ são suficientemente grande.

Até o momento as discussões foram voltadas para as condições necessárias que $\boldsymbol{x}^{*}$ deve satisfazer para ser um mínimo local do problema P. Já as condições suficientes para que $\boldsymbol{x}^{*}$ seja um mínimo local isolado do problema P é

$$
\left\langle\boldsymbol{x}, \nabla^{2} L\left(\boldsymbol{x}^{*}, \boldsymbol{u}^{*}\right) \boldsymbol{x}\right\rangle>0
$$


para todo $\boldsymbol{x} \neq \mathbf{0}$ e satisfazendo as condições

$$
\begin{aligned}
& \left\langle\nabla g_{i}\left(\boldsymbol{x}^{*}\right), \boldsymbol{x}\right\rangle=0 ; \quad i \in E \cup I \\
& \left\langle\nabla g_{i}\left(\boldsymbol{x}^{*}\right), \boldsymbol{x}\right\rangle \leq 0 ; \quad i \notin E \cup I
\end{aligned}
$$

onde $\nabla^{2} L\left(\boldsymbol{x}^{*}, \boldsymbol{u}^{*}\right)$ é a matriz Hessiana do Lagrangeano. Se $\nabla^{2} L\left(\boldsymbol{x}^{*}, \boldsymbol{u}^{*}\right)$ for definida positiva, tem-se uma condição suficiente para que $\boldsymbol{x}^{*}$ seja um mínimo local isolado. Observe que se as condições (3.28) e (3.29) não forem satisfeitas, então $\boldsymbol{x}^{*}$ não é um mínimo local isolado, mas pode ser um mínimo local [11]. Considerando-se que $\boldsymbol{x}^{k}$, para um $k$ suficientemente grande, satisfaz as condições de (3.21) à (3.23), então a condição suficiente para que $\boldsymbol{x}^{k}$ seja um mínimo de $\Phi\left(\boldsymbol{x}, \boldsymbol{u}^{*}, \boldsymbol{r}\right)$ é que $\nabla^{2} \Phi\left(\boldsymbol{x}^{k}, \boldsymbol{u}^{k}, \boldsymbol{r}\right)$ seja definida positiva.

O parâmetro de penalidade $\boldsymbol{r}$ é incrementado de modo a tornar $\nabla^{2} \Phi\left(\boldsymbol{x}^{*}, \boldsymbol{u}^{*}, \boldsymbol{r}\right)$ definida positiva. Segundo [11], se $\nabla^{2} L\left(\boldsymbol{x}^{*}, \boldsymbol{u}^{*}\right)$ é definida positiva, então $\nabla^{2} \Phi\left(\boldsymbol{x}^{*}, \boldsymbol{u}^{*}, \boldsymbol{r}\right)$ também o é, podendo-se finalmente concluir que o mínimo do problema $\mathrm{P}$ é também o mínimo de $\lim _{k \rightarrow \infty} \Phi\left(\boldsymbol{x}^{k}, \boldsymbol{u}^{k}, \boldsymbol{r}\right)$.

\subsubsection{PROPRIEDADES DE CONVERGÊNCIA}

A convergência global do método dos multiplicadores foi estabelecida introduzindo-se procedimentos automáticos para atualização dos parâmetros de penalidade $\boldsymbol{r}_{i, i}=1, m[\mathbf{1 1}]$. Entende-se por convergência global o algoritmo convergir para um mínimo local, adotando-se um valor arbitrário inicial das variáveis de projeto $\boldsymbol{x}$. Isto não implica convergir para um mínimo global. Para o método de penalidade, em [11] mostra-se que existe $\boldsymbol{r}^{0}$ não negativo e $M \in(0, \infty)$ tal que

$$
\left\|\boldsymbol{x}^{k}-\boldsymbol{x} *\right\| \leq \frac{M}{\left\|\boldsymbol{r}^{k}\right\|}\left\|\boldsymbol{u}^{k}-\boldsymbol{u}^{*}\right\|
$$

$\mathrm{e}$

$$
\left\|\boldsymbol{u}^{k+1}-\boldsymbol{u}^{*}\right\| \leq \frac{M}{\left\|\boldsymbol{r}^{k}\right\|} \| \boldsymbol{u}^{k}-\boldsymbol{u}^{* \|}
$$

para todo $r_{i}^{k}>r_{i}^{0}>0$ e $\boldsymbol{u}^{k} \in T$, onde $T$ é uma esfera aberta centrada em $\boldsymbol{u}^{*}$. As expressões (3.30) e (3.31) são utilizadas para se mostrar a convergência global do método dos multiplicadores. As únicas considerações são que $\left\|\boldsymbol{r}^{k}\right\|>M$ e $r_{i}^{k}>r_{i}^{0}>0$ para todo $k$. A taxa de convergência do método dos multiplicadores é criticamente dependente do procedimento de acréscimo dos multiplicadores de Lagrange. $\mathrm{O}$ procedimento dado por (3.13) apresenta convergência linear [11]. 


\subsubsection{O ALGORITMO DO MÉTODO DOS MULTIPLICADORES}

Antes de descrever o algoritmo, efetuar-se-ão algumas observações referentes à sua implementação:

\section{Observação 3.1}

Apenas restrições violadas, ativas ou $\varepsilon$-ativas (Polak [8]) são consideradas em cada iteração.

\section{Observação 3.2}

Como afirmado anteriormente, as propriedades de convergência do método dos multiplicadores são altamente dependente dos procedimentos de acréscimo dos multiplicadores. Uma maneira comum é implementar o método do Lagrangeano aumentado para um valor fixado dos multiplicadores e então incrementa-los.

Um procedimento de acréscimo pode ser geralmente definido como

com a propriedade

$$
\boldsymbol{u}^{k+1}=\boldsymbol{U}\left(\boldsymbol{x}^{k}, \boldsymbol{u}^{k}, \boldsymbol{r}^{k}\right)
$$

$$
\boldsymbol{u}^{*}=\boldsymbol{U}\left(\boldsymbol{x}^{*}, \boldsymbol{u}^{*}, \boldsymbol{r}^{*}\right)
$$

onde $\left(x^{*}, \boldsymbol{u}^{*}\right)$ é a solução do problema. Se $\boldsymbol{U}$ não depende explicitamente de $\boldsymbol{u}$, i.e.

$$
\frac{\partial \boldsymbol{U}}{\partial u_{i}}(\boldsymbol{x}, \boldsymbol{u}, \boldsymbol{r})=\mathbf{0}, \quad i=1, m
$$

então $\boldsymbol{U}$ é denominado Procedimento para Aproximação dos Multiplicadores de Lagrange. Diversos procedimentos para acréscimo dos multiplicadores tem sido apresentado na literatura, e podem ser encontrados em diversos livros e artigos (Arora, Chahande e Paeng [11], Luemberg [5], Fletcher [25], Polak [8], e outros). Neste trabalho utiliza-se o seguinte:

$$
U_{H P}(x, u, r)=u+R g
$$

A matriz $\boldsymbol{R}$ é diagonal, sendo o parâmetro de penalidade $r_{i}$ o $i$-ésimo elemento da sua diagonal principal e o vetor $\boldsymbol{g}=\left[g_{i}(\boldsymbol{x})\right]$ com $i=1, m$. Observa-se que a equação de (3.35) utiliza apenas informações sobre as funções restrições $g_{i}(\boldsymbol{x})$. Segundo [11], na literatura esta expressão é creditada à Hestenes e Powell, e por isso denominada por Procedimento de Hestenes-Powell (UHP) para Acréscimo dos Multiplicadores. Rockafellar e Schuldt independentemente sugeriram modificações à fórmula para restrições de desigualdade. 
Baseando-se nas considerações e estudos anteriores pode-se estabelecer o algoritmo abaixo.

\section{Algoritmo II:}

Passo 1. Faça $k=0 ; K=\infty$; adote os vetores $\boldsymbol{x}^{(0)}, \boldsymbol{\theta}^{(0)}, \boldsymbol{r}$, e os escalares $\alpha>1, \beta>1, \varepsilon>0(\varepsilon$ é um número pequeno usado como critério de parada).

Passo 2. Minimize $\Phi\left(\boldsymbol{x}, \boldsymbol{\theta}^{(k)}, \boldsymbol{r}^{(k)}\right)$ em relação a $\boldsymbol{x}$. Denomina-se $\boldsymbol{x}^{(k)}$ o ponto que minimiza $\Phi\left(\boldsymbol{x}, \boldsymbol{\theta}^{(k)}, \boldsymbol{r}^{(k)}\right)$.

Passo 3. Calcule $g_{i}\left(\boldsymbol{x}^{(k)}\right) ; i=1, m$. Calcule $K_{b}$ e verifique o critério de parada; isto é: se $K_{b}<\varepsilon$, então pare o processo iterativo, caso contrário estabeleça os seguintes conjuntos de restrições de igualdade e desigualdade

$$
\begin{aligned}
& I_{E}=\left\{i:\left|g_{i}\left(\boldsymbol{x}^{(k)}\right)\right|>K / \alpha ; i=1, l\right\} \quad \text { (igualdades) } \\
& I_{I}=\left\{i:\left|\max \left(g_{i}\left(\boldsymbol{x}^{(k)}\right),-\theta_{i}\right)\right|>K / \alpha ; i=l+1, m\right\} \quad \text { (desigualdades) }
\end{aligned}
$$

Passo 4. Efetue os seguintes acréscimos nos parâmetros de penalidade e multiplicadores.

(a) Se $K_{b} \geq K$, faça $r_{i}=\beta r_{i}$ e $\theta_{i}^{(k+1)}=\theta_{i}^{(k)} / \beta$ para todo $i \in I_{E} \cup I_{I}$ isto é incremente os parâmetros de penalidade sem modificar os multiplicadores de Lagrange. Vá ao passo 5.

(b) Se $K_{b}<K$, acresce-se $\theta_{i}^{(k)}$ fazendo

$$
\begin{aligned}
& \theta_{i}^{(k+1)}=\theta_{i}^{(k)}+g_{i}\left(\boldsymbol{x}^{(k)}\right) ; \quad i=1, l \\
& \theta_{i}^{(k+1)}=\theta_{i}^{(k)}+\max \left(g_{i}\left(\boldsymbol{x}^{(k)}\right),-\theta_{i}^{(k)}\right) ; i=l+1, m
\end{aligned}
$$

Vá ao passo 5 .

(c) Se $K b \leq K / \alpha$, faça $K=K b$ e vá ao passo 5. Senão, faça $r_{i}=\beta r_{i}$ e $\theta_{i}^{(k+1)}=\theta_{i}^{(k+1)} / \beta$. para todo $i \in I_{E} \cup I_{I}$. Faça $K=K_{b}$ e vá ao passo 5.

Passo 5. Faça $k=k+1$, e vá passo 2.

Por sugestão de Arora, Chahande e Paeng [11] adota-se os valores: $\theta=\mathbf{0}, \alpha$ e $\beta$ respectivamente igual a 3 e 6 , e $\varepsilon$ igual 0.001 . 


\subsubsection{O MÉTODO DO LAGRANGEANO AUMENTADO EM PROBLEMAS COM RESTRIÇÕES DINÂMICAS}

Como apresentado na seção 3.2, o presente trabalho destina-se à resolução de problemas de otimização com restrições dinâmicas. No que segue, apresentar-se-á uma extensão do desenvolvimento descrito para restrições estáticas para problemas com restrições dinâmicas.

Novamente se apresenta a seguinte formulação: determine $\boldsymbol{x} \in \boldsymbol{R}^{\boldsymbol{n}}$ que minimize a função objetivo $f(\boldsymbol{x})$ sujeito a

Restrições estáticas:

$$
\begin{aligned}
& g_{i}(\boldsymbol{x})=0 ; \quad i=1, l \\
& g_{i}(\boldsymbol{x}) \leq 0 ; \quad i=l+1, m
\end{aligned}
$$

Restrições dinâmicas:

$$
\begin{aligned}
& \text { para todo } t \in\left[t_{\mathrm{o}, t f}\right. \\
& g_{i}(\boldsymbol{x}, t)=g_{i}(\boldsymbol{x}, \boldsymbol{z}(t), \boldsymbol{z}(t), t)=0 ; \quad i=m+1, l^{\prime} \\
& g_{i}(\boldsymbol{x}, t)=g_{i}(\boldsymbol{x}, \boldsymbol{z}(t), \boldsymbol{z}(t), t) \leq 0 ; \quad i=l^{\prime}+1, m^{\prime}
\end{aligned}
$$

$\boldsymbol{z}(t)$ é o vetor das variáveis de estado que se supõe funções contínuas de $t$ no intervalo $\left[t_{0}, t_{f}\right]$, onde $t_{\mathrm{o}}$ e $t_{f}$ são respectivamente os instantes inicial e final.

As variáveis de estado $\boldsymbol{z}(t)$ devem satisfazer o sistema de equações diferenciais de segunda ordem:

$$
\boldsymbol{M}(\boldsymbol{x}) \ddot{\boldsymbol{z}}(t)+\boldsymbol{C}(\boldsymbol{x}, \dot{z}(t)) \dot{\boldsymbol{z}}(t)+\boldsymbol{R}(\boldsymbol{x}, \boldsymbol{z}(t))=\boldsymbol{p}(\boldsymbol{x}, t)
$$

com as condições iniciais $\dot{\boldsymbol{z}}\left(t_{0}\right)=\dot{\boldsymbol{z}}_{0}$ e $\boldsymbol{z}\left(t_{0}\right)=\boldsymbol{z}_{0}$, onde $\boldsymbol{M ( x )}$ e $\boldsymbol{C}(\boldsymbol{x}, \dot{\boldsymbol{z}}(t))$ são respectivamente as matrizes de massa e de amortecimento. $\boldsymbol{R}(\boldsymbol{x}, \boldsymbol{z}(t))$ é o vetor força restauradora. Já $\boldsymbol{p}(\boldsymbol{x}, t)$ é o vetor das forças generalizado.

Pode-se encontrar um sistema de equações diferenciais de primeira ordem equivalente à (3.40) e então utilizar algoritmos de primeira ordem para resolvê-lo. 
As restrições com respostas dinâmicas são funções explicitas das variáveis de estado e implícitas das variáveis de projeto. Para avalia-las é necessário resolver o sistema (3.40) que precisa ser integrado numericamente. Para isso o intervalo $\left[t_{\mathrm{o}}, t_{f}\right]$ é discretizado. $\mathrm{O}$ valor das funções restrições são calculados nos pontos da discretização. Funções de interpolação são utilizadas para se determinar valores intermediários de $g_{i}$.

Neste caso, as funções restrições dinâmicas são integradas ao longo do intervalo de tempo e combinadas com a função objetivo para se obter o funcional. Este funcional pode ser interpretado como o Lagrangeano do problema que é aumentado com funções de penalidade. Os multiplicadores são adotados inicialmente e, como no caso estático, o Lagrangeano aumentado é minimizado. Os multiplicadores são então acrescidos e o procedimento repetido. Supõe-se, nos exemplos analisados neste trabalho, que $r_{i}$ e $\theta_{i}$ não dependem do tempo.

Para a otimização do problema com respostas dinâmicas, definido pelas equações (3.36) à (3.40), o Lagrangeano aumentado é definido por

$$
\begin{gathered}
\Phi(\boldsymbol{x}, \boldsymbol{\theta}, \boldsymbol{r})=f(\boldsymbol{x})+\frac{1}{2}\left\{\sum_{i=1}^{l} r_{i}\left(g_{i}(\boldsymbol{x})+\theta_{i}\right)^{2}+\sum_{i=l+1}^{m} r_{i}\left(g_{i}(\boldsymbol{x})+\theta_{i}\right)_{+}^{2}\right\}+ \\
\frac{1}{2} \int_{t_{0}}^{t_{f}}\left\{\sum_{i=m+1}^{l^{\prime}} r_{i}\left(g_{i}(\boldsymbol{x}, t)+\theta_{i}\right)^{2}+\sum_{i=l^{\prime}+1}^{m^{\prime}} r_{i}\left(g_{i}(\boldsymbol{x}, t)+\theta_{i}\right)_{+}^{2}\right\} d t
\end{gathered}
$$

Onde $r_{i} \theta_{i}=u i, i=1, m$ ', são os multiplicadores de Lagrange. Ainda $\theta_{i} \geq 0$ para $i=l+1, m \mathrm{e}$ $\theta_{i} \geq 0$ para $i=l^{\prime}+1, m^{\prime} . r_{i}(>0)$ é o parâmetro de penalidade correspondente à restrição $g_{i}$, para $i=1, m$ '.

\section{PROCEDIMENTO COMPUTACIONAL}

Para se elaborar o algoritmo dos multiplicadores para problema com resposta dinâmica, o parâmetro $K_{b}$ é definido como

Define-se a máxima violação de restrição $K_{b} \geq 0$, para um dado $\boldsymbol{x}^{(k)}$, como:

$$
\begin{aligned}
& K_{b}=\max \left\{\max _{1 \leq i \leq l}\left|g_{i}\left(\boldsymbol{x}^{(k)}\right)\right| ; \max _{l+1 \leq i \leq m}\left|\max \left(g_{i}\left(\boldsymbol{x}^{(k)}\right),-\theta_{\mathrm{i}}\right)\right| ;\right. \\
& \left.\max _{m+1 \leq i \leq l^{\prime}}\left(\max _{t_{0} \leq t \leq t_{f}}\left|g_{i}\left(\boldsymbol{x}^{(k)}, t\right)\right|\right) ; \max _{l^{\prime}+1 \leq i \leq m^{\prime}}\left(\max _{t_{0} \leq t \leq t_{f}}\left|\max \left(g_{i}\left(\boldsymbol{x}^{(k)}, t\right),-\theta_{i}(t)\right)\right|\right)\right\}
\end{aligned}
$$


Passo 1. Faça $k=0 ; K_{b}=\infty$; estime os vetores $\boldsymbol{x}^{(0)}, \theta^{(0)}, \boldsymbol{r}$, e os escalares $\alpha>1, \beta>1, \varepsilon>0($ $\varepsilon$ é um número pequeno usado como critério de parada).

Passo 2. Minimize $\Phi\left(\boldsymbol{x}, \boldsymbol{\theta}^{(k)}, \boldsymbol{r}^{(k)}\right)$ em relação a $\boldsymbol{x}$. Denomina-se $\boldsymbol{x}^{(k)}$ o ponto que minimiza $\Phi\left(\boldsymbol{x}, \boldsymbol{\theta}^{(k)}, \boldsymbol{r}^{(k)}\right)$.

Passo 3. Calcule $g_{i}\left(\boldsymbol{x}^{(k)}\right), i=1, m$ e $g_{i}\left(\boldsymbol{x}^{(k)}, t\right) ; i=m+1, m^{\prime}$ e $t \in\left[t_{0}, t_{f}\right]$. Calcule $K_{b}$ e cheque o critério de parada; isto é: se $K_{b}<\varepsilon$, então pare. Caso contrário estabeleça os seguintes conjuntos de restrições de igualdade e desigualdade

$$
\begin{aligned}
& I_{E}=\left\{i:\left|g_{i}\left(\boldsymbol{x}^{(k)}\right)\right|>K / \alpha ; i=1, l\right\} \quad \text { (igualdades) } \\
& I_{I}=\left\{i:\left|\max \left(g_{i}\left(\boldsymbol{x}^{(k)}\right),-\theta_{i}\right)\right|>K / \alpha ; i=l+1, m\right\} \quad \text { (desigualdades) } \\
& I_{E^{\prime}}=\left\{i: \max _{t}\left|g_{i}\left(\boldsymbol{x}^{(k)}, t\right)\right|>K / \alpha ; i=m+1, l^{\prime}\right\} \quad \text { (igualdades dinâmicas) } \\
& I_{I^{\prime}}=\left\{i: \max _{t} \mid \max \left(g_{i}\left(\boldsymbol{x}^{(k)}, t\right),-\theta_{i}^{(k)}(t) \mid>K / \alpha ; i=l^{\prime}+1, m^{\prime}\right\} \quad\right. \text { (desigualdades dinâmicas) }
\end{aligned}
$$

Passo 4. Efetue os seguintes acréscimos nos parâmetros de penalidade e multiplicadores.

(a) Se $K_{b} \geq K$, faça $r_{i}=\beta r_{i}$ e $\theta_{i}^{(k+1)}=\theta_{i}^{(k)} / \beta$ para todo $i \in I_{E} \cup I_{I}$; faça $r_{i}=\beta r_{i}$ e $\theta_{i}^{(k+1)}(t)=\theta_{i}^{(k)}(t) / \beta$ para todo $i \in I_{E^{\prime}} \cup I_{I^{\prime}}$ e $t \in\left[t_{o}, t_{f}\right]$; isto é incremente os parâmetros de penalidade sem modificar os multiplicadores de Lagrange. Vá ao passo 5.

(b) Se $K_{b}<K$, acresce-se $\theta_{i}^{(k)}$ fazendo

$$
\begin{aligned}
& \theta_{i}^{(k+1)}=\theta_{i}^{(k)}+g_{i}\left(\boldsymbol{x}^{(k)}\right) ; \quad i=1, l \\
& \theta_{i}^{(k+1)}=\theta_{i}^{(k)}+\max \left(g_{i}\left(\boldsymbol{x}^{(k)}\right), \quad-\theta_{i}^{(k)}\right) ; \quad i=l+1, m \\
& \theta_{i}^{(k+1)}(t)=\theta_{i}^{(k)}(t)+g_{i}\left(\boldsymbol{x}^{(k)}, t\right) ; \quad i=m+1, l^{\prime}, t \in\left[t_{0}, t_{f}\right] \\
& \theta_{i}^{(k+1)}(t)=\theta_{i}^{(k)}(t)+\max \left(g_{i}\left(\boldsymbol{x}^{(k)}, t\right), \quad-\theta_{i}^{k}(t)\right) ; i=l^{\prime}+1, m^{\prime}, t \in\left[t_{0}, t_{f}\right]
\end{aligned}
$$

e vá ao passo 5 .

(c) Se $K_{b} \leq K / \alpha$, faça $K=K_{b}$ e vá ao passo 5. Senão, faça $r_{i}=\beta r_{i}$ e $\theta_{i}^{(k+1)}=\theta_{i}^{(k+1)} / \beta \quad$ para todo $i \in I_{E} \cup I_{I}$; faça $r_{i}=\beta r_{i}$ e $\theta_{i}^{(k+1)}(t)=\theta_{i}^{(k+1)}(t) / \beta$.para todo $i \in I_{E^{\prime}} \cup I_{I^{\prime}}$ e $t \in\left[t_{0}, t_{f}\right]$. Faça $K=K b$ e vá ao passo 5 . 
Passo 5. Faça $k=k+1$, e vá passo 2 .

Na $k$-ésima iteração, uma vez obtida a solução $\boldsymbol{x}^{(k)}$ no passo 3 , se o critério de parada não for satisfeito, os parâmetros de penalidade são acrescidos e o processo repetido. 


\section{Capítulo 4}

\section{MÉTODOS NUMÉRICOS}

Para se implementar computacionalmente o Método dos multiplicadores é necessário a programação de diversos métodos numéricos. Esta seção é destinada a um breve resumo dos seguintes métodos numéricos:

1. Na resolução dos sistemas lineares utiliza-se decomposição de Cholesky;

2. Para se determinar as integrais de (3.42), efetua-se interpolação via splines cúbicos e a seguir integração com quadratura de Gauss-Legendre. Para efeito de comparação utiliza-se também a regra do trapézio no cálculo destas integrais;

3. Na resolução das equações do movimento utiliza-se os métodos de RungeKutta de quarta e quinta ordem e o método de Newmark;

4. Relativamente à minimização sem restrições (passo 2) utiliza-se o método dos gradientes com busca unidimensional de Armijo;

5. E finalmente o cálculo do gradiente do Lagrangeano aumentado é efetuado utilizando diferenças finitas, uma vez que nem todas as variáveis aparecem explicitamente. 


\subsection{SOLUÇÃO DE SISTEMAS LINEARES}

Os sistemas lineares em engenharia desempenham papel importante, já que tais sistemas constituem, segundo Calaes [2], a aproximação mais simples para interpretação matemática de fenômenos originalmente muito complexos.

Considere então o sistema de equações

$$
A \boldsymbol{x}=\boldsymbol{b}
$$

onde $\boldsymbol{A}=\left[\begin{array}{cccc}a_{11} & a_{12} & \cdots & a_{1 n} \\ a_{21} & a_{22} & \cdots & a_{2 n} \\ \vdots & \vdots & & \vdots \\ a_{n 1} & a_{n 2} & \cdots & a_{n n}\end{array}\right]$ e $\boldsymbol{b}=\left[\begin{array}{c}b_{1} \\ b_{2} \\ \vdots \\ b_{n}\end{array}\right]$ são dados, e $\boldsymbol{x}=\left[\begin{array}{c}x_{1} \\ x_{2} \\ \vdots \\ x_{n}\end{array}\right]$ é a incógnita.

Existem dois grandes grupos de métodos para se determinar $\boldsymbol{x}$ em (4.1): Os Métodos Diretos e os Iterativos. Os métodos diretos fornecem a solução exata do sistema linear em um número finito de passos. No presente trabalho trataremos apenas o método direto de Decomposição de Choleski, usual nos casos em que $\boldsymbol{A}$ é definida positiva.

O teorema de Choleski, cuja demonstração encontra-se em Goldenberg e Pimenta [19], é o seguinte: se $\boldsymbol{A}$ é definida positiva, então existe $\boldsymbol{R}$ triangular superior com diagonal positiva tal que $\boldsymbol{A}=\boldsymbol{R}^{T} \boldsymbol{R}$ e que esta decomposição é única. Ainda em [19] é apresentado a seguinte solução:

$$
\begin{aligned}
& R_{i i}=\left[A_{i i}-\left(R_{1 i}^{2}+R_{2 i}^{2}+\ldots+R_{(i-1) i}^{2}\right)\right]^{\frac{1}{2}} \\
& R_{i j}=\left[A_{i j}-\left(R_{1 i} R_{1 j}+R_{2 i} R_{2 j}+\ldots+R_{(i-1) i} R_{(i-1) j}\right)\right] / R_{i i}
\end{aligned}
$$

onde $i=1, \ldots, n$ e $j=i+1, \ldots, n$. Observa-se que uma ordem conveniente para se resolver as equações (4.2) é $R_{11}, R_{12}, \ldots, R_{1 n}, R_{22}, R_{23}, \ldots, R_{2 n}, R_{33}, \ldots, R_{n n}$.

Uma vez obtido $\boldsymbol{R}$ a solução do sistema $\boldsymbol{A} \boldsymbol{x}=\boldsymbol{b}$ fica reduzida à solução de dois sistemas triangulares 


$$
\boldsymbol{R}^{T} \boldsymbol{y}=\boldsymbol{b} \text { e } \boldsymbol{R} \boldsymbol{x}=\boldsymbol{y}
$$

Para se resolver (4.3) utiliza-se os procedimentos

$$
\begin{aligned}
& y_{i}=\left[b_{i}-\left(R_{1 i} y_{1}+R_{2 i} y_{2}+\ldots+R_{(i-1) i} y_{(i-1)}\right)\right] / R_{i i} ; i=1, \ldots, n \\
& x_{i}=\left[y_{i}-\left(R_{i n} x_{n}+R_{i(n-1)} x_{(n-1)}+\ldots+R_{i(i+1)} x_{(i+1)}\right)\right] / R_{i i} ; i=n, \ldots, 1
\end{aligned}
$$

Um caso particular importante que será bastante utilizado neste trabalho é o caso em que $\boldsymbol{A}$ é tridiagonal. Neste caso a decomposição de Choleski se resume a

$$
\begin{aligned}
& R_{i i}=\left[A_{i i}-R_{(i-1) i}^{2}\right]^{\frac{1}{2}}, \quad i=1, \ldots, n \\
& R_{i(i+1)}=A_{i(i+1)} / R_{i i}, \quad i=1, \ldots, n-1
\end{aligned}
$$

e (4.4) resulta em

$$
\begin{gathered}
y_{i}=\left[b_{i}-R_{(i-1) i} y_{(i-1)}\right] / R_{i i} ; \quad i=1, \ldots, n \\
x_{i}=\left[y_{i}-R_{i(i+1)} x_{(i+1)}\right] / R_{i i} ; \quad i=n, \ldots, 1
\end{gathered}
$$

\subsection{MÉTODOS DE INTEGRAÇÃO NUMÉRICA}

\subsubsection{INTRODUÇÃO}

Seja $S:[a, b] \rightarrow \mathbf{R}$ e

$$
I(S)=\int_{a}^{b} S(x) d x
$$

sua integral definida. Prova-se que

$$
I(S)=P S(b)-P S(a)
$$


onde $P S(x)$ é a função primitiva de $S(x)$. A expressão (4.8) é conhecida como Teorema Fundamental do Cálculo. Muitas integrais podem ser calculadas usando essa fórmula.

Existe uma variada gama de livros destinados a tratar este assunto, um bom exemplo é a obra de Piskunov [16]. Porém, muitas integrais não podem ser resolvidas usando (4.8) porque seus integrandos podem não ter primitivas expressas em termos de funções elementares, como por exemplo $\int_{0}^{1} e^{x^{2}} d x$ e $\int_{0}^{\pi} x \operatorname{sen}(\sqrt{x}) d x$.

Posto isso, é necessário desenvolver outros métodos para resolver estas integrais. Os Métodos de Integração Numérica são uma maneira computacionalmente interessante de resolver este problema.

Existem diversos métodos de integração numérica. Dentre eles pode-se destacar os Métodos Newton-Cotes e a Quadratura de Gauss. Sobre os métodos Newton-Cotes é feito uma rápida explanação na seção 4.2.2, maiores detalhes são encontrados em Chapra e Canale [26]. A quadratura de Gauss, utilizando a base polinomial ortonormal de Legendre, é descrita na seção 4.2.3. Segundo Atkinson [12], os métodos da quadratura de Gauss são na sua maioria superiores em precisão do que os Newton-Cotes.

\subsubsection{FÓRMULAS DE INTEGRAÇÃO NEWTON-COTES}

Para facilitar o cálculo de $I(S)$, substitui-se $S(x)$ pelo polinômio $S_{n}(x)$ de ordem $n$, da forma

$$
S_{n}(x)=a_{0}+a_{1} x+\ldots+a_{n-1} x^{n-1}+a_{n} x^{n}
$$

Um bom exemplo deste método é o da figura 4.1a onde se substitui o integrando $S(x)$ por uma reta (processo de integração denominado regra do trapézio) e na figura $4.1 \mathrm{~b}$ por uma parábola (integração pela regra de Simpson 1/3).

Observando-se a figura 4.1a, deduz-se que

$$
I=\int_{a}^{b} S(x) d x \cong(b-a) \frac{S(a)+S(b)}{2}
$$


sendo que o erro de truncamento, ou seja, o erro cometido utilizando-se este método, segundo Chapra e Canale [26], é

$$
E_{t}=-\frac{1}{12} S^{\prime \prime}(\xi)(b-a)^{3}
$$

onde $\xi$ é um ponto do intervalo $[a, b]$.

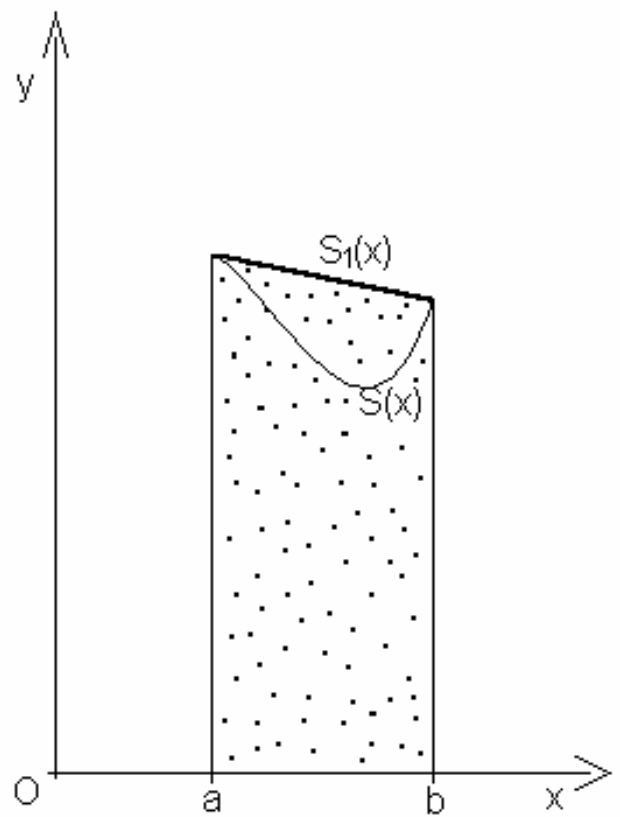

[a]

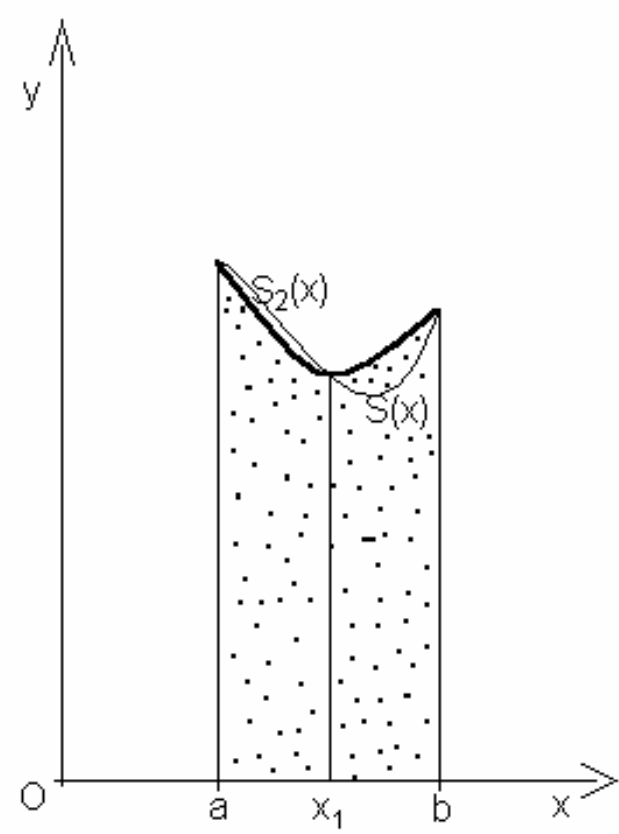

[b]

Figura 4.1. Integração pelo método Newton-Cotes para $n=1,2$.

Nestes métodos o segmento $[a, b]$ é subdividido em $n$ trechos de comprimento $h$ e $S_{n}(x)$ é a interpolação polinomial nos pontos $x_{i}=a+i h, i=0, n$. Demonstra-se, Johnson e Riess [13], que por $n+1$ pontos existe um e somente um polinômio interpolador de ordem $n$. A tabela 4.1 contém as fórmula de Newton-Cotes para aproximações com polinômios de ordem 1,2 e 3 .

Observa-se que nas fórmulas de Newton-Cotes os valores de $S(x)$ são requeridos somente nos pontos $x_{i}=a+i h, i=0, n$. Na seção seguinte trata-se os métodos de quadratura de 
Gauss onde a diferença básica com os Newton-Cotes é que o valor de $S(x)$ é requerido em pontos que não são necessariamente $x_{i}=a+i h, i=0, n$.

TABELA 4.1 Fórmulas de Newton-Cotes e seus respectivos erros.

\begin{tabular}{|c|c|c|c|c|}
\hline Segmentos (n) & $\begin{array}{c}\text { Números de } \\
\text { Pontos }\end{array}$ & Nome & Fórmula & $\begin{array}{c}\text { Erro de } \\
\text { Truncamento }\end{array}$ \\
\hline 1 & 2 & $\begin{array}{c}\text { Regra do } \\
\text { Trapézio }\end{array}$ & $(b-a) \frac{S\left(x_{0}\right)+S\left(x_{1}\right)}{2}$ & $-\frac{1}{12} h^{3} S^{\prime \prime}(\xi)$ \\
\hline 2 & 3 & $\begin{array}{c}\text { Regra de } \\
\text { Simpson } 1 / 3\end{array}$ & $(b-a) \frac{S\left(x_{0}\right)+4 S\left(x_{1}\right)+S\left(x_{2}\right)}{6}$ & $-\frac{1}{90} h^{5} S^{(4)}(\xi)$ \\
\hline 3 & 4 & $\begin{array}{c}\text { Regra de } \\
\text { Simpson 3/8 }\end{array}$ & $(b-a) \frac{S\left(x_{0}\right)+3 S\left(x_{1}\right)+3 S\left(x_{2}\right)+S\left(x_{3}\right)}{8}$ & $-\frac{3}{80} h^{5} S^{(4)}(\xi)$ \\
\hline
\end{tabular}

\subsubsection{QUADRATURA DE GAUSS}

A característica dessas fórmulas é que elas não fixam os pontos $x_{j}, j=0, \ldots, n-1$ do domínio onde é requerido o valor de $S(x)$, consequentemente pode-se escolher pontos que permitam obter um valor mais preciso para a integral.

O método de quadratura de Gauss foi desenvolvido para integrais mais gerais, onde uma função peso $w(x)$ também pode ser considerada. Esta generalização inclui a possibilidade de se tratar integrais de funções com singularidades. Neste trabalho não aparecem integrais com singularidades e se adotará $w(x)=1$. Entretanto para não fugir da descrição padrão da literatura o método será descrito para uma função peso $w(x)$. Segundo Johnson e Riess [13] a função peso deve satisfazer as seguintes condições:
i) assumir valores positivos em $[a, b]$;
ii) ser contínua em $[a, b]$;
iii) $\int_{a}^{b} w(x) d x$ deve existir e ser maior que zero.

Considera-se então

$$
I=\int_{a}^{b} w(x) S(x) d x
$$


A idéia central da quadratura de Gauss consiste em determinar pesos $w_{i}$ e abscissas $x_{i}$, $i=0, \ldots, n-1, n \in \mathrm{Z}$ (utilizando polinômios de Gauss-Legendre que são tratados nesta seção) de modo que a aproximação

$$
\int_{a}^{b} w(x) S(x) d x \cong \sum_{j=0}^{n-1} w_{j} S\left(x_{j}\right)
$$

seja exata se $S(x)$ for um polinômio de grau até $2 n-1$. O segundo membro de (4.13) é denominado de Quadratura de Gauss e usualmente se escreve

$$
Q(S)=\sum_{j=0}^{n-1} w_{j} S\left(x_{j}\right)
$$

Antes de se passar à descrição do método é necessário apresentar algumas definições e efetuar-se algumas considerações.

Como mencionado no capítulo 3, analogamente ao espaço vetorial $\mathbf{R}^{n}$ pode-se definir o produto escalar no espaço das funções contínuas. Dado duas funções $f(x)$ e $g(x)$, seu produto escalar em $[a, b]$, com peso $w(x)$, é definido como $\left\langle f, g>=\int_{a}^{b} w(x) f(x) g(x) d x\right.$. Se esta integral for nula, diz-se que as funções $f(x)$ e $g(x)$ são ortogonais entre si em $[a, b]$. Um conjunto de funções que são mutuamente ortogonais e individualmente normalizadas, ou seja, possuem módulo unitário, é denominado de base polinomial ortonormal.

Uma família de polinômios $P=\left\{p_{j}, j=0,1,2, \ldots\right\}$ é dita ortonormal se $\left\langle p_{i}, p_{j}\right\rangle=\delta_{i j}, i, j=0,1, \ldots, n$.

Pode-se gerar diversas famílias de polinômios ortonormais como por exemplo as de GaussLegendre, Gauss-Chebyshev, Gauss-Laguerre, Gauss-Hermite e Gauss-Jacobi. Johnson e Riess [13] demonstram que estas famílias constituem uma base do espaço de funções. Neste trabalho estuda-se apenas as soluções de Gauss-Legendre.

A base polinomial ortonormal de Gauss-Legendre é gerada por 


$$
\begin{gathered}
w(x)=1 \quad-1<x<1 \\
p_{0}=1 \\
p_{1}=x \\
(j+1) p_{j+1}=(2 j+1) x p_{j}-j p_{j-1}
\end{gathered}
$$

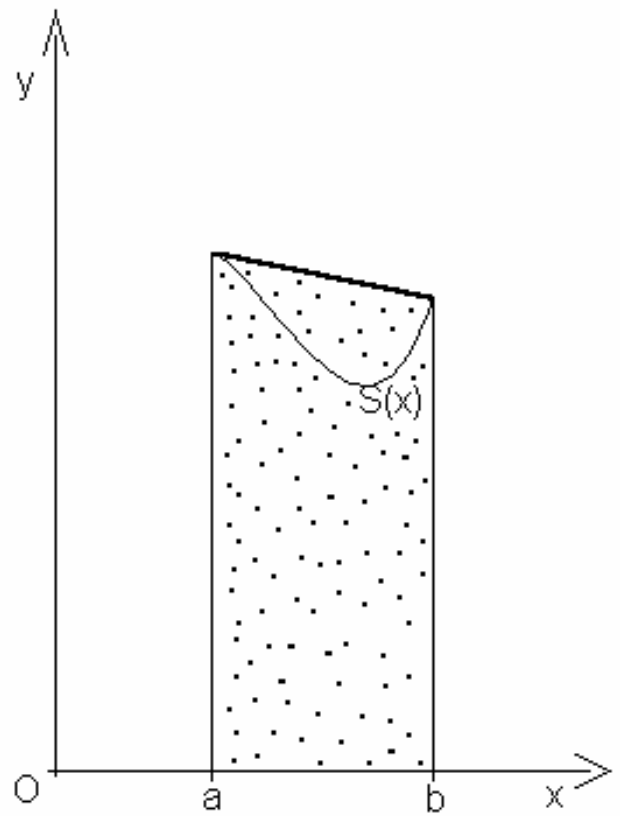

(a)Regra do Trapézio

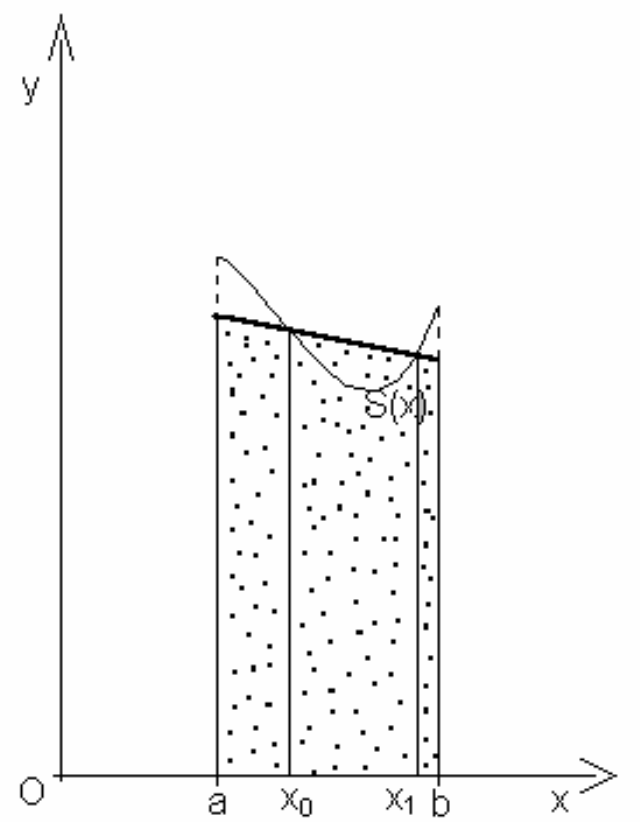

(b) Quadratura de Gauss

Figura 4.2. Comparação geométrica entre a Regra do Trapézio e a Quadratura de Gauss.

Johnson e Riess [13] provam também que as raízes do n-ésimo polinômio de GaussLegendre são as abscissas $x_{i} \operatorname{dos} n$ pontos da fórmula da quadratura de Gauss. Este resultado é conhecido como Teorema da Quadratura de Gauss.

Após determinado as $n$ raízes de $p_{n}(x)$, calcula-se

$$
w_{j}=\frac{2}{\left(1-x_{j}^{2}\right)\left[p_{n}^{\prime}\left(x_{j}\right)\right]^{2}}
$$


Observa-se que o domínio de integração para os polinômios de Gauss-Legendre é $-1 \leq x_{d} \leq 1$ e na integral (4.12) é $a \leq x \leq b$. Então é necessário substituir nesta equação $x=[(b-$ a) $/ 2] x_{d}+(b+a) / 2$.

TABELA 4.2 Pesos $w$ e argumentos $x$ utilizados nas fórmulas de Gauss-Legendre.

\begin{tabular}{|c|c|c|c|}
\hline Pontos (n) & Fator Peso & $\begin{array}{c}\text { Argumentos da } \\
\text { Função }\end{array}$ & $\begin{array}{c}\text { Erro de } \\
\text { Truncamento }\end{array}$ \\
\hline 2 & $w_{0}=1,000000000$ & $x_{0}=-0,577350269$ & $\cong S^{(4)}(\xi)$ \\
& $w 1=1,000000000$ & $x_{1}=0,577350269$ & \\
\hline 3 & $w_{0}=0,555555556$ & $x_{0}=-0,774596669$ & $\cong S^{(6)}(\xi)$ \\
& $w 1=0,888888889$ & $x_{1}=0,000000000$ & \\
& $w_{2}=0,555555559$ & $x_{2}=0,774596669$ & $\cong S^{(8)}(\xi)$ \\
& $w 0=0,347854845$ & $x_{0}=-0,861136312$ & \\
& $w 1=0,652145155$ & $x_{1}=-0,339981044$ & \\
& $w_{2}=0,652145155$ & $x_{2}=0,339981044$ & \\
& $w_{3}=0,347854845$ & $x_{3}=0,861136312$ & \\
\hline
\end{tabular}

A tabela 4.2 mostra, para vários valores de $n$, os fatores $w$ e os argumentos $x$ da quadratura de Gauss para os polinômios de Gauss-Legendre. Uma tabela para $n=5,6$ pode ser encontrada em Chapra e Canale [26].

\subsection{INTERPOLAÇÃO POLINOMIAL}

\subsubsection{INTRODUÇÃO}

Um problema comumente encontrado em trabalhos científicos é a aproximação de funções "de difícil trato", $f(x)$, por funções "de fácil trato", $p(x)$. Neste trabalho, em particular esta aproximação será utilizada para efetuarmos a integração da função $f(x)$ utilizando os métodos de quadratura de Gauss. Um modo usual de se aproximar uma função é o polinômio de Taylor de ordem $k$,

$$
p_{k}(x)=f(a)+f^{\prime}(a)(x-a)+\frac{f^{\prime \prime}(a)(x-a)^{2}}{2 !}+\ldots+\frac{f^{(k)}(a)(x-a)^{k}}{k !},
$$

que aproxima $f(x)$ em torno de $x=a$. Se $f^{(k+1)}(x)$ for contínua, então o erro de aproximação é dado por $\frac{f^{(k+1)}(\xi)(x-a)^{k+1}}{(k+1) !}$, onde $\xi$ é um ponto entre $x$ e $a$. A implementação desta aproximação pode trazer complicações analíticas quando, por exemplo, as derivadas da 
função $f(x)$ não existirem ou forem de difícil cálculo, ou quando os erros de truncamento forem consideráveis.

Um dos principais objetivos ao se analisar a aproximação de funções é obter procedimentos computacionais de fácil implementação. Nos trabalhos de Atkinson [12], Chapra e Canale [26] e Johnson e Riess [13] os métodos mais discutidos são a interpolação polinomial, a aproximação de Fourrier e a interpolação com splines cúbicos. Neste trabalho trata-se apenas de interpolação usando funções spline.

Ao se executar uma aproximação é preciso estabelecer mecanismos para avalia-la. Pode-se dizer que $p(x)$ é uma boa aproximação para $f(x)$ se a norma da função $f(x)-p(x)$ for pequena no intervalo $[a, b]$. As normas mais usuais são

$$
\begin{array}{r}
\|f-p\|_{1}=\int_{a}^{b}|f(x)-p(x)| w(x) d x, \\
\|f-p\|_{2}=\left(\int_{a}^{b}(f(x)-p(x))^{2} w(x) d x\right)^{\frac{1}{2}} \mathrm{e} \\
\|f-p\|_{\infty}=\max _{a \leq x \leq b}|f(x)-p(x)| .
\end{array}
$$

Neste contexto, considera-se que a função a ser aproximada, $f(x)$, é contínua no intervalo fechado $[a, b]$, ou seja, $f(x) \in C_{1}[a, b]$. A função $w(x)$ é denominada função peso. Como descrito na seção 4.2.3, a função $w(x)$ deve satisfazer as seguintes condições:

$$
\begin{aligned}
& \text { i) assumir valores positivos em }[a, b] \text {; } \\
& \text { ii) ser contínua em }[a, b] \\
& \text { iii) } \int_{a}^{b} w(x) d x \text { deve existir e ser maior que zero. }
\end{aligned}
$$

\subsubsection{INTERPOLAÇÃO COM FUNÇÕES SPLINE CÚBICOS}

\section{Definição 4.1:}


Uma função $f(x)$ é interpolada nos pontos $x_{i}, i=0, \ldots, n$-1, pela função $S(x)$, onde $f(x)$ e $S(x) \in$ $C_{1}[a, b]$, se e somente se $S\left(x_{i}\right)=f\left(x_{i}\right)$, para $i=0, \ldots, n-1$.

A interpolação de $f(x)$ por $S(x)$ é um processo que define uma função $S(x)$ que tem imagem comum $\operatorname{com} f(x) \operatorname{nos}$ pontos $x_{i}, i=0, \ldots, n-1$.

Considere um conjunto de $n$ pontos $\left(x_{i}, y_{i}\right), i=0, \ldots, n-1$, onde $y_{i}=S\left(x_{i}\right)$. Visando simplificações considera-se que

$$
x_{0}<x_{1}<x_{2}<\ldots<x_{n-2}<x_{n-1}
$$

$a=x_{0} \quad$ e $\quad b=x_{n-1}$. Deseja-se obter uma função $S_{i}(x) \in C_{2}[a, b]$, onde $\left\{x_{i-1} \leq x \leq x_{i}\right\}$ e $i=1, \ldots, n-1$, tal que

$$
S\left(x_{i}\right)=y_{i}, \quad i=0, \ldots, n-1
$$

Para que $S(x) \in C_{2}[a, b]$ é necessário que $S^{\prime}(x)$ e $S^{\prime \prime}(x)$ sejam contínuas em $[a, b]$.

Atkinson [12] mostra que o problema de se determinar $S(x)$ pode ser colocado na forma:

S1. $S(x)$ é um polinômio de grau $\leq 3$ em cada subintervalo $\left[x_{i-1}, x_{i}\right], i=1, \ldots, n-1$;

S2. $S(x), S^{\prime}(x)$ e $S^{\prime \prime}(x)$ são contínuas para $a \leq x \leq b$;

S3. $S^{\prime \prime}\left(x_{0}\right)=S^{\prime \prime}\left(x_{n-1}\right)=0$.

A solução deste problema, é a função $S(x)=\left\{S_{i}(x)\right.$, onde $\left\{x_{i-1} \leq x \leq x_{i}\right\}$ e $\left.i=1, \ldots, n-1\right\}$, sendo que $S_{i}(x)$ é dada pela expressão

$$
\begin{gathered}
S_{i}(x)=\frac{\left(x_{i}-x\right)^{3} M_{i-1}+\left(x-x_{i-1}\right)^{3} M_{i}}{6\left(x_{i}-x_{i-1}\right)}+\frac{\left(x_{i}-x\right) y_{i-1}+\left(x-x_{i-1}\right) y_{i}}{x_{i}-x_{i-1}} \\
-\frac{1}{6}\left(x_{i}-x_{i-1}\right)\left[\left(x_{i}-x\right) M_{i-1}+\left(x-x_{i-1}\right) M_{i}\right]
\end{gathered}
$$

onde $M_{i}$ é

$$
M_{i}=S^{\prime \prime}\left(x_{i}\right), i=0, \ldots, n-1
$$

Determina-se $M_{i}$ através das equações 


$$
\frac{x_{i}-x_{i-1}}{6} M_{i-1}+\frac{x_{i+1}-x_{i-1}}{3} M_{i}+\frac{x_{i+1}-x_{i}}{6} M_{i+1}=\frac{y_{i+1}-y_{i}}{x_{i+1}-x_{i}}-\frac{y_{i}-y_{i-1}}{x_{i}-x_{i-1}}
$$

onde $i=1, \ldots, n-2, \mathrm{e}$

$$
M_{0}=M_{n-1}=0 \text {. }
$$

O sistema linear (4.25) é tridiagonal e simétrico, e pode ser resolvido facilmente. Existem algoritmos exclusivos para sistemas tridiagonais.

Se o espaçamento entre os pontos $x_{i}, i=0, \ldots, n-1$, for constante igual a $h$, a matriz dos coeficientes de (4.25) é definida positiva e constante para um dado $n$, como se observa nas expressões (4.27) e (4.28).

$$
M_{i-1}+4 M_{i}+M_{i+1}=\frac{6}{h^{2}}\left(y_{i+1}-2 y_{i}+y_{i-1}\right), \text { onde } i=1, \ldots, n-2
$$

ou na forma matricial

$$
\left[\begin{array}{ccccccc}
4 & 1 & 0 & \cdots & 0 & 0 & 0 \\
1 & 4 & 1 & \cdots & 0 & 0 & 0 \\
0 & 1 & 4 & \cdots & 0 & 0 & 0 \\
\vdots & \vdots & \vdots & \vdots & \vdots & \vdots & \vdots \\
0 & 0 & 0 & \cdots & 4 & 1 & 0 \\
0 & 0 & 0 & \cdots & 1 & 4 & 1 \\
0 & 0 & 0 & \cdots & 0 & 1 & 4
\end{array}\right]\left[\begin{array}{c}
M_{1} \\
M_{2} \\
M_{3} \\
\vdots \\
M_{n-4} \\
M_{n-3} \\
M_{n-2}
\end{array}\right]=\frac{6}{h^{2}}\left[\begin{array}{c}
y_{0}-2 y_{1}+y_{2} \\
y_{1}-2 y_{2}+y_{3} \\
y_{2}-2 y_{3}+y_{4} \\
\vdots \\
y_{n-5}-2 y_{n-4}+y_{n-3} \\
y_{n-4}-2 y_{n-3}+y_{n-2} \\
y_{n-3}-2 y_{n-2}+y_{n-1}
\end{array}\right]
$$

E a solução $S(x)=\left\{S_{i}(x)\right.$, onde $\left\{x_{i-1} \leq x \leq x_{i}\right\}$ e $\left.i=1, \ldots, n-1\right\}$ resulta em

$$
\begin{gathered}
S_{i}(x)=\frac{\left(x_{i}-x\right)^{3} M_{i-1}+\left(x-x_{i-1}\right)^{3} M_{i}}{6 h}+\frac{\left(x_{i}-x\right) y_{i-1}+\left(x-x_{i-1}\right) y_{i}}{h}, \\
-\frac{1}{6} h\left[\left(x_{i}-x\right) M_{i-1}+\left(x-x_{i-1}\right) M_{i}\right]
\end{gathered},
$$

onde $i=1, \ldots, n-2$. 


\subsection{MÉTODOS DE SOLUÇÃO DE SISTEMA DE EQUAÇÕES DIFERENCIAIS ORDINÁRIAS DE PRIMEIRA E SEGUNDA ORDEM}

\subsubsection{INTRODUÇÃO}

Alguns problemas de Dinâmica Estrutural são regidos por sistemas de equações diferenciais ordinárias, ver Burnett [6]. Neste capítulo realiza-se uma breve revisão sobre sistemas de equações diferenciais ordinárias de $1^{a}$ e $2^{a}$ ordem e se apresenta métodos numéricos para resolvê-los. Nos sistemas de segunda ordem analisa-se apenas o caso de equações diferenciais lineares com coeficientes constantes, enquanto que para os de primeira ordem são apresentados métodos mais gerais.

\subsubsection{MÉTODOS RUNGE-KUTTA DE QUARTA E QUINTA ORDEM}

As equações diferenciais ordinárias de primeira ordem analisadas neste estudo são consideradas normais, e consequentemente podem ser colocadas na forma

$$
z^{\prime}(x)=f(x, z(x)), \quad x \geq x_{0}
$$

onde $z(x)$ é a função a ser determinada. $z\left(x_{o}\right)$ é denominado valor inicial de $z(x)$. A função dada $f(x, z((x))$ define a equação diferencial que pode ser linear ou não. (4.30) é denominada de primeira ordem porque contém a função incógnita com derivadas de primeira ordem. Um sistema de equações diferenciais ordinárias de primeira ordem pode ser definido como

$$
\boldsymbol{z}^{\prime}(x)=\boldsymbol{f}(x, \boldsymbol{z}(x)), \quad x \geq x_{0}
$$

$\boldsymbol{z}(x)$ é um vetor $n$-dimensional, $x$ a variável independente e $f(x,(z(x))$ uma função vetorial $n$ dimensional. Para que a equação (4.31) apresente uma única solução, o valor de $z(x)$ deve ser conhecido em $x=x_{o}($ Piskunov [16]).

Atkinson [12] mostra que uma equação de ordem maior que um pode ser reformulada e apresentada como um sistema de equações de primeira ordem. Então os métodos numéricos deste item podem ser estendidos a este novo sistema. 
Considere $x \in\left[x_{0}, x_{m-1}\right]$. Este intervalo pode ser dividido em $m$-1 intervalos, na forma, $\left[x_{0}, \ldots, x_{k-1}, x_{k}, x_{k+1}, \ldots, x_{m-1}\right]$, onde a distância entre dois pontos consecutivos é uma constante de valor igual a $h$. Neste contexto o método Runge-Kutta de quarta ordem pode ser resumido pelas expressões

$$
\boldsymbol{z}\left(x_{k+1}\right)=\boldsymbol{z}\left(x_{k}\right)+\frac{\boldsymbol{k}_{1}+2 \boldsymbol{k}_{2}+2 \boldsymbol{k}_{3}+\boldsymbol{k}_{4}}{6}
$$

onde

$$
\begin{aligned}
& \boldsymbol{k}_{1}=h \boldsymbol{f}\left(\boldsymbol{z}\left(x_{k}\right), x_{k}\right), \\
& \boldsymbol{k}_{2}=h \boldsymbol{f}\left(\boldsymbol{z}\left(x_{k}\right)+0.5 \boldsymbol{k}_{1}, x_{k}+0.5 h\right), \\
& \boldsymbol{k}_{3}=h \boldsymbol{f}\left(z\left(x_{k}\right)+0.5 \boldsymbol{k}_{2}, x_{k}+0.5 h\right) \mathrm{e} \\
& \boldsymbol{k}_{4}=h \boldsymbol{f}\left(\boldsymbol{z}\left(x_{k}\right)+\boldsymbol{k}_{3}, x_{k}+h\right) .
\end{aligned}
$$

O método Runge-Kutta de quinta ordem pode ser descrito por

$$
\boldsymbol{z}\left(x_{k+1}\right)=\boldsymbol{z}\left(x_{k}\right)+\frac{23 \boldsymbol{k}_{1}+125 \boldsymbol{k}_{3}-81 \boldsymbol{k}_{5}+125 \boldsymbol{k}_{6}}{192}
$$

sendo que

$$
\begin{aligned}
& \boldsymbol{k}_{1}=h \boldsymbol{f}\left(\boldsymbol{z}\left(x_{k}\right), x_{k}\right), \\
& \boldsymbol{k}_{2}=h \boldsymbol{f}\left(\boldsymbol{z}\left(x_{k}\right)+\frac{\boldsymbol{k}_{1}}{3}, x_{k}+\frac{h}{3}\right), \\
& \boldsymbol{k}_{3}=h \boldsymbol{f}\left(\boldsymbol{z}\left(x_{k}\right)+\frac{6 \boldsymbol{k}_{2}+4 \boldsymbol{k}_{1}}{25}, x_{k}+0.4 h\right), \\
& \boldsymbol{k}_{4}=h \boldsymbol{f}\left(\boldsymbol{z}\left(x_{k}\right)+\frac{15 \boldsymbol{k}_{3}-12 \boldsymbol{k}_{2}+\boldsymbol{k}_{1}}{4}, x_{k}+h\right), \\
& \boldsymbol{k}_{5}=h \boldsymbol{f}\left(\boldsymbol{z}\left(x_{k}\right)+\frac{8 \boldsymbol{k}_{4}-50 \boldsymbol{k}_{3}+90 \boldsymbol{k}_{2}+6 \boldsymbol{k}_{1}}{81}, x_{k}+\frac{2 h}{3}\right) \mathrm{e} \\
& \boldsymbol{k}_{6}=h \boldsymbol{f}\left(\boldsymbol{z}\left(x_{k}\right)+\frac{8 \boldsymbol{k}_{4}+10 \boldsymbol{k}_{3}+36 \boldsymbol{k}_{2}+6 \boldsymbol{k}_{1}}{75}, x_{k}+0.8 h\right) .
\end{aligned}
$$


Observa-se que nas equações (4.32) à (4.35) não há nenhuma exigência sobre linearidade de $f(z(x), x)$.

\subsubsection{MÉTODO DE NEWMARK}

Pode-se definir um sistema de equações diferenciais ordinárias lineares de $2^{a}$ ordem pela equação

$$
\boldsymbol{D} \boldsymbol{z}^{\prime \prime}(x)+\boldsymbol{E} \boldsymbol{z}^{\prime}(x)+\boldsymbol{F} \boldsymbol{z}(x)=\boldsymbol{g}(x)
$$

onde $\boldsymbol{D}, \boldsymbol{E}$ e $\boldsymbol{F}$ são matrizes quadradas que não dependem da variável independente $x$, enquanto que $\boldsymbol{z}$ e $\boldsymbol{g}$ são matrizes colunas (vetores) funções de $x$. Em dinâmica estrutural a variável independente é o tempo e comumente representada pela letra $t$.

Uma solução do sistema de equações definido por (4.36) é uma função $z(x)$ contínua com derivadas contínuas até $2^{a}$ ordem que o satisfaça.

Para se obter uma única solução em (4.36) é necessário que sejam dadas as condições iniciais

$$
\boldsymbol{z}\left(x_{0}\right)=\boldsymbol{z}_{0} \text { e } \boldsymbol{z}^{\prime}\left(x_{0}\right)=\boldsymbol{z}_{0}^{\prime} .
$$

Genericamente o problema de se determinar $z(x)$, satisfazendo as condições iniciais, é denominado por Problema de Valor Inicial.

Existem dois principais grupos de método numéricos para se resolver um problema de valor inicial regido por um sistema de equações diferenciais de segunda ordem: os Métodos de Integração Direta e os Métodos de Superposição Modal. No presente trabalho trata-se apenas o Método Newmark (método de integração direta) que é descrito a seguir.

Para seguir a descrição clássica da literatura, nas próximas expressões deste item substituiremos a variável $x$ pela variável $t$. As primeira e segunda derivadas de $z(t) \mathrm{em}$ relação à $t$ serão representadas respectivamente por $\dot{\boldsymbol{z}}(t)$ e $\ddot{\boldsymbol{z}}(t)$.

Em alguns problemas de dinâmica estrutural o problema definido por (4.36) e (4.37) aparece como descrito a seguir. 
Equação do Movimento:

$$
\boldsymbol{M} \ddot{\boldsymbol{z}}(t)+\boldsymbol{C} \dot{\boldsymbol{z}}(t)+\boldsymbol{K} \boldsymbol{z}(t)=\boldsymbol{f}(t)
$$

Domínio Temporal:

$$
t_{0} \leq t \leq t_{m-1}
$$

Condições Iniciais:

$$
\dot{\boldsymbol{z}}\left(t_{0}\right)=\dot{\boldsymbol{z}}_{0} \text { e } \boldsymbol{z}\left(t_{0}\right)=\boldsymbol{z}_{0}
$$

onde $\boldsymbol{M}, \boldsymbol{C}$ e $\boldsymbol{K}$ são, respectivamente, as matrizes de massa, de amortecimento viscoso e de rigidez, $\boldsymbol{f}(t)$ é o vetor força generalizado e $\boldsymbol{z}(t)$ o vetor deslocamento.

A solução numérica para este problema requer que o intervalo $\left[t_{\mathrm{o}}, t_{\mathrm{m}-1}\right]$ seja dividido em $\mathrm{m}$-1 intervalos $\left[t_{0}, \ldots, t_{k-1}, t_{k}, t_{k+1}, \ldots, t_{m-1}\right]$ para que o valor aproximado de $\boldsymbol{z}(t)$ seja calculado nos pontos $t=t_{k}, k=0, \ldots, m-1$. Para simplificar considera-se que a distância entre dois pontos adjacentes é constante igual a $h$. Ou seja $h=t_{k+1}-t_{k}, k=0, \ldots, m-2$.

Segundo Kikuchi [15], um dos métodos mais populares para resolver o problema definido pelas equações (4.38) à (4.40) é o Método de Newmark, que é baseado na aproximação

$$
\begin{aligned}
& \dot{\boldsymbol{z}}_{k+1}=\dot{\boldsymbol{z}}_{k}+h\left[(1-\theta) \ddot{\boldsymbol{z}}_{k}+\theta \ddot{\boldsymbol{z}}_{k+1}\right] \\
& \boldsymbol{z}_{k+1}=\boldsymbol{z}_{k}+h \dot{\boldsymbol{z}}_{k}+\frac{1}{2} h^{2} \ddot{\boldsymbol{z}}_{k}+\beta h^{2}\left(\ddot{\boldsymbol{z}}_{k+1}-\ddot{\boldsymbol{z}}_{k}\right)
\end{aligned}
$$

Onde $\boldsymbol{z}_{k}$ é o valor da função $\boldsymbol{z}(t)$ em $t=t_{k}$. Se os parâmetros $\theta$ e $\beta$ são tomados respectivamente como $1 / 2$ e $1 / 4$, tem-se estabilidade incondicional (isto é, os erros numéricos não aumentam com o passar do tempo de execução), Kikuchi [15].

Para $k=0$, é conhecido $\boldsymbol{z}_{k}$ e $\dot{\boldsymbol{z}}_{k}$, de (4.38) obtem-se $\ddot{\boldsymbol{z}}_{k}$. Portanto para determinar $\dot{\boldsymbol{z}}_{k+1}$ em (4.41) e $\boldsymbol{z}_{k+1}$ em (4.42) é necessário o cálculo de $\ddot{\boldsymbol{z}}_{k+1}$. Para obter este vetor substitui-se (4.41) e (4.42) em (4.38) para $t=t_{k+1}$, obtendo-se:

$$
\begin{gathered}
\left(\boldsymbol{M}+\theta h \boldsymbol{C}+\beta h^{2} \boldsymbol{K}\right) \ddot{\boldsymbol{z}}_{k+1}=\boldsymbol{f}\left(t_{k+1}\right)-\boldsymbol{K} \boldsymbol{z}_{k}-(\boldsymbol{C}+h \boldsymbol{K}) \dot{\boldsymbol{z}}_{k} \\
-\left[(1-\theta) h \boldsymbol{C}+\left(\frac{1}{2}-\beta\right) h^{2} \boldsymbol{K}\right] \ddot{\boldsymbol{z}}_{k}
\end{gathered}
$$


Denominando-se

$$
\begin{aligned}
& \boldsymbol{A}=\boldsymbol{M}+\theta h \boldsymbol{C}+\beta h^{2} \boldsymbol{K} \mathrm{e} \\
& \boldsymbol{b}\left(t_{k+1}\right)=\boldsymbol{f}\left(t_{k+1}\right)-\boldsymbol{K} \boldsymbol{z}_{\boldsymbol{k}}-(\boldsymbol{C}+h \boldsymbol{K}) \dot{\boldsymbol{z}}_{k}-\left[(1-\theta) h \boldsymbol{C}+\left(\frac{1}{2}-\beta\right) h^{2} \boldsymbol{K}\right] \ddot{\boldsymbol{z}}_{k}
\end{aligned}
$$

Obtém-se

$$
\boldsymbol{A} \ddot{\boldsymbol{z}}_{k+1}=\boldsymbol{b}\left(t_{k+1}\right)
$$

Resolve-se (4.45) obtendo-se $\ddot{\boldsymbol{z}}_{k+1}$, e se substituindo em (4.41) e (4.42) obtém-se respectivamente $\dot{\boldsymbol{z}}_{k+1}$ e $\boldsymbol{z}_{k+1}$.

Observa-se que $\boldsymbol{A}$ é constante para $t_{0} \leq t \leq t_{m-1}$ e que em dinâmica estrutural $\boldsymbol{A}$ é também definida positiva. Posto isso, é comum utilizar-se decomposição de Choleski para resolver (4.45).

Repetindo-se o processo acima para $k=1, \ldots, m-1$ obtém-se os valores de $\boldsymbol{z}_{k}, \dot{\boldsymbol{z}}_{k}$ e $\ddot{\boldsymbol{z}}_{k}$, tabelados nos pontos $t=t_{k}, k=0, \ldots, m-1$.

\subsection{ALGORITMO DE MINIMIZAÇÃO SEM RESTRIÇÕES}

Considere o problema de minimizar a função continuamente diferenciável $f: \boldsymbol{R}^{n} \rightarrow \boldsymbol{R}$. O problema pode ser escrito na forma compacta

$$
\min \left\{f(\boldsymbol{x}) \mid \boldsymbol{x} \in \boldsymbol{R}^{n}\right\}
$$

Supõe-se que seja possível encontrar $\boldsymbol{x}_{0} \in \boldsymbol{R}^{n}$ tal que o conjunto

$$
C\left(x_{0}\right)=\left\{x \mid f(x) \leq f\left(x_{0}\right)\right\}
$$


seja limitado.

Os algoritmos que resolvem o problema definido por (4.46) buscam pontos $\boldsymbol{x}^{\prime} \in \boldsymbol{R}^{n}$ tal que $\nabla f\left(\boldsymbol{x}^{\prime}\right)=\mathbf{0}$. Diz-se então que $\boldsymbol{x}^{\prime}$ é um ponto desejável.

Para uma direção $\boldsymbol{h}\left(\boldsymbol{x}_{i}\right)$ e um ponto $\boldsymbol{x}_{i}$ do espaço $\boldsymbol{R}^{n}$, e $\boldsymbol{D}\left(\boldsymbol{x}_{i}\right)$ uma matriz $n_{\mathrm{x}} n$ definida positiva cujos elementos são funções contínuas e diferenciáveis em $\boldsymbol{x}$, um algoritmo que minimiza $f(\boldsymbol{x})$ pode ser apresentado na forma:

Passo 0. Calcule $\boldsymbol{x}_{0} \in \boldsymbol{R}^{n}$ tal que o conjunto definido em (3.107) seja limitado.

Passo 1. Coloque $i=0$.

Passo 2. Calcule a direção $\boldsymbol{h}\left(\boldsymbol{x}_{i}\right)=-\boldsymbol{D}\left(\boldsymbol{x}_{i}\right) \nabla f\left(\boldsymbol{x}_{i}\right)$. Se $\boldsymbol{h}\left(\boldsymbol{x}_{i}\right)=\mathbf{0}$ pare, caso contrário vá ao passo 3 .

Passo 3. Calcule o escalar $\lambda\left(\boldsymbol{x}_{i}\right)$ como sendo o menor escalar não negativo satisfazendo

$$
f\left(\boldsymbol{x}_{i}+\lambda\left(\boldsymbol{x}_{i}\right) \boldsymbol{h}\left(\boldsymbol{x}_{i}\right)\right)=\min \left\{f\left(\boldsymbol{x}_{i}+\lambda \boldsymbol{h}\left(\boldsymbol{x}_{i}\right)\right) \mid \lambda \geq 0\right\}
$$

Passo 4. Coloque $\boldsymbol{x}_{i+1}=\boldsymbol{x}_{i}+\lambda\left(\boldsymbol{x}_{i}\right) \boldsymbol{h}\left(\boldsymbol{x}_{i}\right)$, faça $i=i+1$ e vá ao passo 2 .

Um caso particular do algoritmo conceitual que será utilizado neste trabalho é o método dos gradientes, onde a matriz $\boldsymbol{D}=\boldsymbol{I}$ ( $\boldsymbol{I}$ é a matriz identidade). Segundo Polak [8] este método gera uma classe de algoritmos com convergência de primeira ordem.

Para o para o cálculo de $\lambda\left(\boldsymbol{x}_{i}\right)$ no passo 3 do algoritmo acima apresenta-se a regra de Armijo:

Defini-se $\theta(\mu, \boldsymbol{x})=[f(\boldsymbol{x}+\mu \boldsymbol{h}(\boldsymbol{x}))-f(\boldsymbol{x})]-\mu \alpha<\nabla f(\boldsymbol{x}), \boldsymbol{h}(\boldsymbol{x})>$. Considere $\boldsymbol{x} i, \alpha \in(0,1)$, $\beta \in(0,1), \rho>0$.

Passo 1. Coloque $\mu=\rho$.

Passo 2. Calcule $\theta\left(\mu, \boldsymbol{x}_{i}\right)$.

Passo 3. Se $\theta\left(\mu, \boldsymbol{x}_{i}\right) \leq 0$ coloque $\lambda\left(\boldsymbol{x}_{i}\right)=\mu$ e pare o processo. Caso contrário coloque $\mu=\beta \mu$ e vá ao passo 2 . 


\subsection{MÉTODO DAS DIFERENÇAS FINITAS}

Para calcular numericamente a derivada de uma função $f(x)$ recorda-se primeiramente a definição de derivada:

$$
f^{\prime}(x)=\lim _{h \rightarrow 0} \frac{f(x+h)-f(x)}{h}
$$

Com base nisso, justifica-se definir

$$
f^{\prime}(x) \stackrel{\ominus}{=} \frac{f(x+h)-f(x)}{h} \equiv D_{h} f(x)
$$

para pequenos valores de $h . D_{h} f(x)$ é denominada derivada numérica de $f(x)$ com passo $h$.

De forma semelhante, para uma função $g(\boldsymbol{x})$, onde $\boldsymbol{x}=\left(x_{0}, x_{1}, \ldots, x_{i}, \ldots, x_{n-1}\right)$ é um vetor $n$ dimensional, pode-se definir seu gradiente numérico por

$$
\boldsymbol{h}(\boldsymbol{x}) \equiv \nabla g(\boldsymbol{x}) \stackrel{\ominus}{=}\left[h_{i}(\boldsymbol{x})\right]
$$

onde $h_{i}(\boldsymbol{x})=\frac{g\left(x_{0}, x_{1}, \ldots, x_{i}+h, \ldots, x_{n-1}\right)-g\left(x_{0}, x_{1}, \ldots, x_{i}, \ldots, x_{n-1}\right)}{h}, i=0, \ldots, n-1$ 


\section{Capítulo 5}

\section{EXEMPLOS RESOLVIDOS PELO MÉTODO DOS MULTIPLICADORES}

Em diversos sistemas mecânicos (absorvedores de impactos, isoladores de vibração, sistema de suspensão de automóveis e estruturas solicitadas por impacto) o comportamento do sistema em regime dinâmico precisa ser considerado. Resolver estes problemas pode se tornar complicado, visto que o projetista tem somente um controle indireto sobre a resposta dinâmica, ou seja, o controle é exercido apenas sobre as variáveis de projeto. Estas podem ser modificadas, desde que a solução da equação do movimento apresente valores toleráveis.

Nos sistemas mecânicos analisados, as variáveis de projeto representam tanto constantes elásticas e do amortecimento viscoso quanto valores máximos para acelerações e deslocamentos. As variáveis de estado são os deslocamentos. A função objetivo é função dos deslocamentos ou de suas derivadas segunda em relação ao tempo.

Já em dinâmica estrutural (edifícios solicitados por impactos) as componentes do vetor variável de projeto descrevem por exemplo área da seção transversal, momento de inércia, rigidez, ou outra propriedade geométrica do elemento estrutural. O vetor das variáveis de estado possui como componentes os deslocamentos da estrutura em função do tempo. Os problemas consistem em minimizar a massa da estrutura, impondo-se restrições aos deslocamentos e tensões. Nestes problemas a função objetivo depende somente das variáveis de projeto $\boldsymbol{x}$. 
Exemplos de aplicações são agora formulados em termos de programação matemática utilizando os conceitos descritos nos Capítulos 2 e 3. Estes exemplos são resolvidos com auxílio de um programa de computador desenvovido em linguagem $\mathrm{C}$.

Como já mencionado no Capítulo 4, para se implementar computacionalmente o Método do Lagrangeano Aumentado é necessário a programação dos métodos numéricos descritos a seguir:

- Relativamente à minimização sem restrições (passo 2) utilizou-se o método dos gradientes com busca unidimensional de Armijo. O cálculo do gradiente do Lagrangeano aumentado foi efetuado utilizando diferenças finitas (com passo $h=10^{-6}$ ), uma vez que nem todas as variáveis aparecem explicitamente.

- Para se determinar as integrais de (3.41), utilizou-se dois métodos:

1) Interpolação via splines cúbicos e a seguir integração com quadratura de Gauss-Legendre;

2) Regra do trapézio.

- Na resolução dos sistemas lineares utilizou-se decomposição de Cholesky.

- Finalmente, a integração da equação do movimento foi realizada com os métodos:

1) Método de Newmark quando a equação do movimento for linear;

2) Método Runge-Kutta de quarta ordem;

3) Método Runge-Kutta de quinta ordem.

Analisou-se oito exemplos de dinâmica de sistemas mecânicos e estruturais. A análise consistiu em um estudo comparativo das combinações dos diferentes métodos utilizados (Newmark/Splines, Newmark/Trapézio, Runge-Kutta de Quarta Ordem/Splines, RungeKutta de Quarta Ordem/Trapézio, Runge-Kutta de Quinta Ordem/Splines, Runge-Kutta de Quinta Ordem/Trapézio). Foram adotados dois valores iniciais para as variáveis de projeto $\left(\boldsymbol{x}_{0}\right)$ e dois intervalos $(n-1)$ na discretização do intervalo de tempo para cada combinação dos métodos. Os valores finais das variáveis de projeto $\left(\boldsymbol{x}_{f}\right)$ e da função objetivo $f\left(\boldsymbol{x}_{f}\right)$, bem como o número de minimizações sem restrições $(k)$ obtidos são mostrados nas tabelas e gráficos que comparam o desempenho das diversas combinações de métodos. 


\subsection{EXEMPLO 1 (Pimenta, Goldenberg, Brasil e Silva [24])}

\section{Pórtico Elástico Linear com um grau de liberdade:}

$\mathrm{O}$ caso analisado é o Shear-building da figura 5.1. O pórtico é solicitado pelo impacto descrito pela função força $p(t)$ (figura 5.1b). O modelo matemático utilizado para representar o pórtico é elástico linear.

A massa $M(x)$ é igual a da viga $\mathrm{BC}, 12000 \mathrm{Kg}$, somada a $13 / 35$ da massa dos pilares AB e CD. O fração 13/35 da massa dos pilares é obtido através de uma interpolação cúbica dos deslocamentos nos trechos $\mathrm{AB}$ e $\mathrm{CD}$. As condições iniciais para o deslocamento $z$ são $z(0)$ $=0$ e $z(0)=0$. Assume-se o intervalo de tempo [0,1] em segundos e subdividido em $(n-1)$ intervalos. O material dos pilares é concreto com módulo de elasticidade $E=20 \mathrm{GPa}$ e densidade $\rho=2,5 \mathrm{t} / \mathrm{m}^{3}$. Considera-se atuando nos pilares somente tensão de flexão, cujo valor máximo aceitável é $\sigma_{b}=20 \mathrm{MPa}$. O máximo deslocamento permitido é $z_{b}=0,04 m$ para $t \in[0,1]$. A variável de projeto e a função objetivo são respectivamente o momento de inércia $x$, em $m^{4}$, e a massa dos pilares $f(x)$, em $K g$.

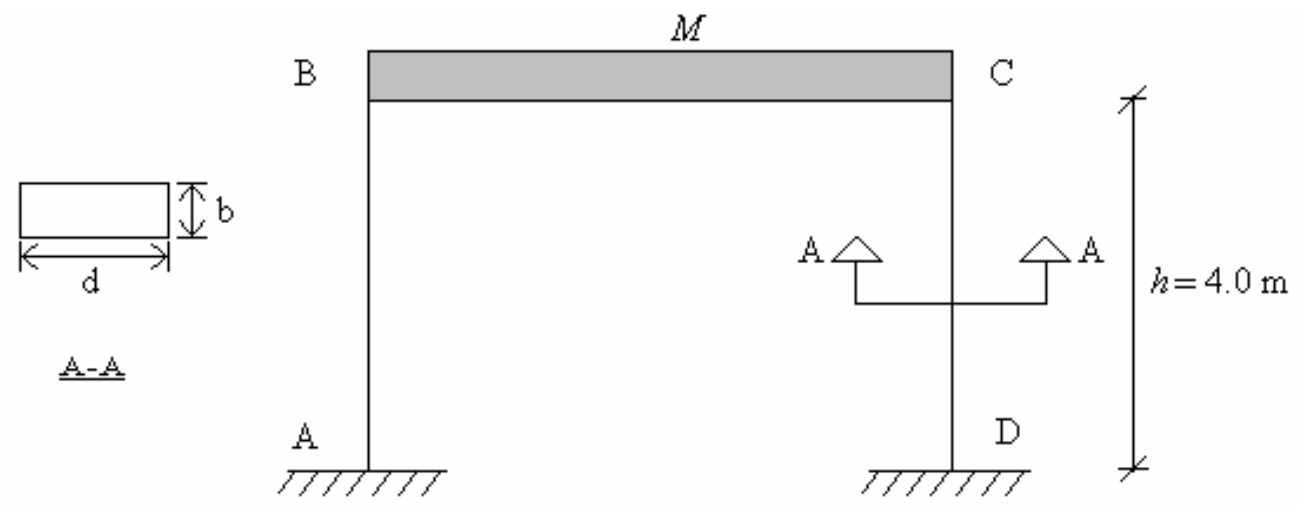

a. Pórtico com um grau de liberdade

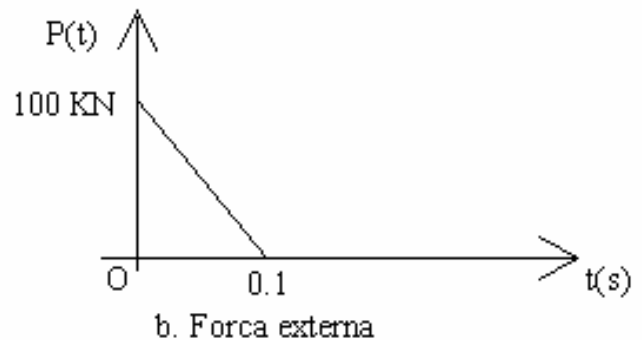

Figura 5.1 Pórtico com um grau de liberdade 
A rigidez dos pilares $K(x)$ em $N / m$, a área da seção transversal dos pilares em $m^{2}$, a massa $M(x)$ em $K g$, e a tensão de flexão em $N / m^{2}$ podem ser calculadas por

$$
\begin{aligned}
& K(x)=7.510^{9} x, \\
& A(x)=0.557 x^{1 / 3}, \\
& M(x)=12000+(13 / 35) 2 \rho 4 A(x) \quad \mathrm{e} \\
& \sigma(x, t)=1.7410^{10} x^{1 / 3} z(t) .
\end{aligned}
$$

O problema de otimização é então

minimizar

$$
f(x)=2 \times 2.5 \times 10^{3} \times 4 A(x),
$$

sujeito a:

$$
\begin{aligned}
& |z(t)|-z_{b} \leq 0, \quad t \in[0,1] \quad \mathrm{e} \\
& |\sigma(x, t)|-\sigma_{b} \leq 0, \quad t \in[0,1] .
\end{aligned}
$$

O valor mínimo da variável $x$ é $0.12 \times 0.12^{3} / 12=1.72810^{-5}$. Impõe-se então a restrição

$$
-x+1.72810^{-5} \leq 0 .
$$

O valor de $z(t)$ é obtido pela integração da equação

$$
M(x) \ddot{z}(t)+K(x) z(t)=p(t),
$$

com as condições iniciais $z(0)=0$ e $z(0)=0$.

Este exemplo foi calculado para dois valores iniciais das variáveis de projeto, o caso 1 que corresponde a um valor inicial de $x$ contido na região viável, e o caso 2 que corresponde a um valor inicial fora da região viável.

O número de 100 subdivisões nas tabelas 5.1 à 5.3 e de 50 subdivisões nas tabelas 5.4 à 5.5 foram adotados na discretização do intervalo de tempo $[0,1] \mathrm{s}$. 
Tabela 5.1: Valores finais das variáveis de projeto para diferentes valores de $x_{0}$

\begin{tabular}{|c|c|c|c|c|c|c|c|}
\cline { 3 - 8 } \multicolumn{1}{c|}{} & \multicolumn{6}{c|}{ Xf.1E4 } \\
\cline { 2 - 8 } \multicolumn{1}{c|}{} & Xo.1E4 & Newm/Splin & Newm/Trap & Rk4/Splin & Rk4/Trap & Rk5/Splin & Rk5/Trap \\
\hline CASO 1 & 10,000 & 16,028 & 16,028 & 10,192 & 10,192 & 10,190 & 10,194 \\
\hline CASO 2 & 0,000 & 16,028 & 16,028 & 10,192 & 10,192 & 10,194 & 10,194 \\
\hline
\end{tabular}

Tabela 5.2: Valores finais da função objetivo para diferentes valores de $x_{\mathrm{o}}$

\begin{tabular}{|c|c|c|c|c|c|c|c|}
\cline { 2 - 8 } \multicolumn{1}{c|}{} & \multicolumn{7}{c|}{$\mathbf{f ( X f )}$} \\
\cline { 2 - 8 } \multicolumn{1}{c|}{} & $\mathbf{f ( X )}$ & Newm/Splin & Newm/Trap & Rk4/Splin & Rk4/Trap & Rk5/Splin & Rk5/Trap \\
\hline CASO 1 & 1114,0 & 1303,7 & 1303,7 & 1121,1 & 1121,1 & 1121,2 & 1121,2 \\
\hline CASO 2 & 0,0 & 1303,7 & 1303,7 & 1121,1 & 1121,1 & 1121,2 & 1121,2 \\
\hline
\end{tabular}

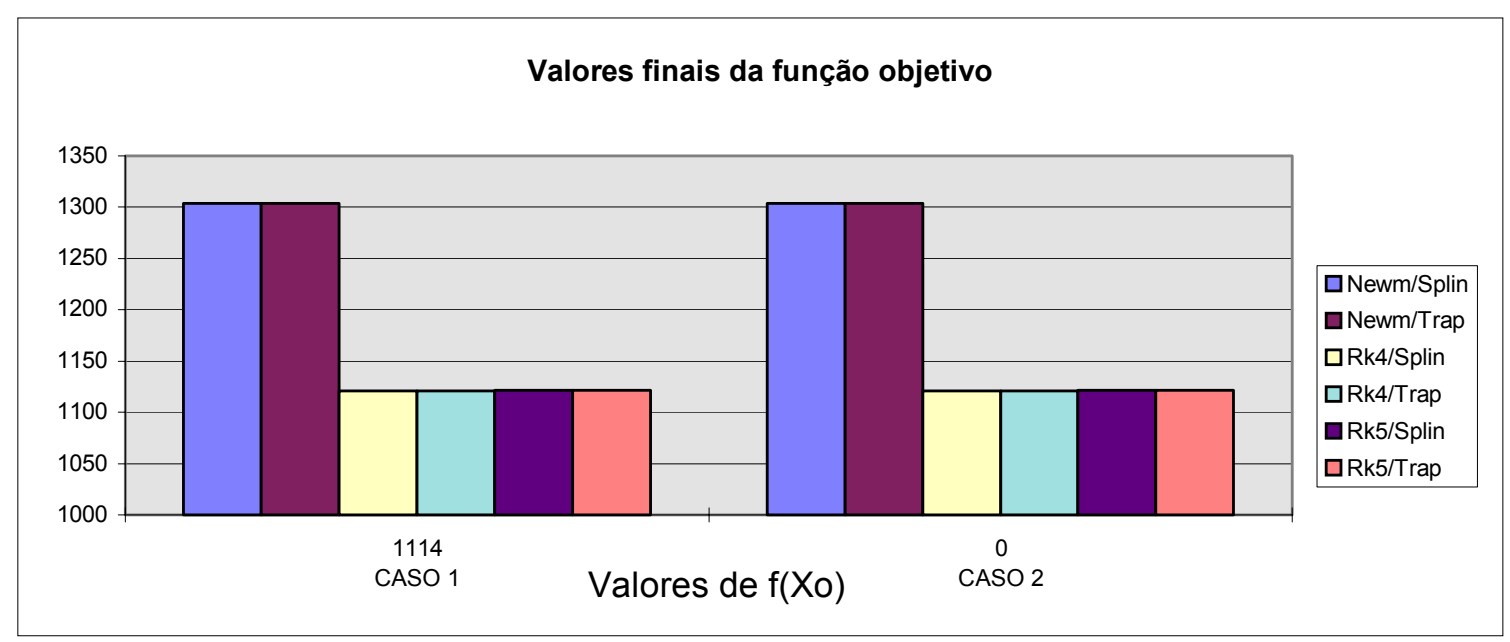

Gráfico 5.1a - Valores finais da função objetivo para diferentes valores de $x_{\mathrm{o}}$ (tabela 5.2).

No gráfico acima observa-se uma diferença de $16 \%$ nas soluções dos métodos de Newmark e Runge-Kutta de quarta e quinta ordem. Visando identificar a origem desta diferença, construiu-se o gráfico 5.1.a com os deslocamentos apresentados pelos métodos de Newmark e Runge-Kutta de quarta e quinta ordem na solução da equação do movimento para $x=0.001 \mathrm{~m}^{4}$. Observa-se neste gráfico uma diferença entre as amplitudes do Método de Newmark e Runge-Kutta de $5 \%$. Este fato mostra uma grande sensibilidade do Método do Lagrangeano Aumentado em relação à variação da solução da equação do movimento. Por outro lado, o valor final da função objetivo apresentou valores bem próximos tanto no caso 1 quanto no caso 2 para todas as combinações dos métodos.

Já no gráfico 5.2, observa-se que o número de minimizações sem restrições foi maior para o caso 2 , ou seja para um valor inicial de $x$ fora da região viável., porém o valor final da função objetivo permanece praticamente inalterado para os dois casos, como já verificado no gráfico 5.1a. 


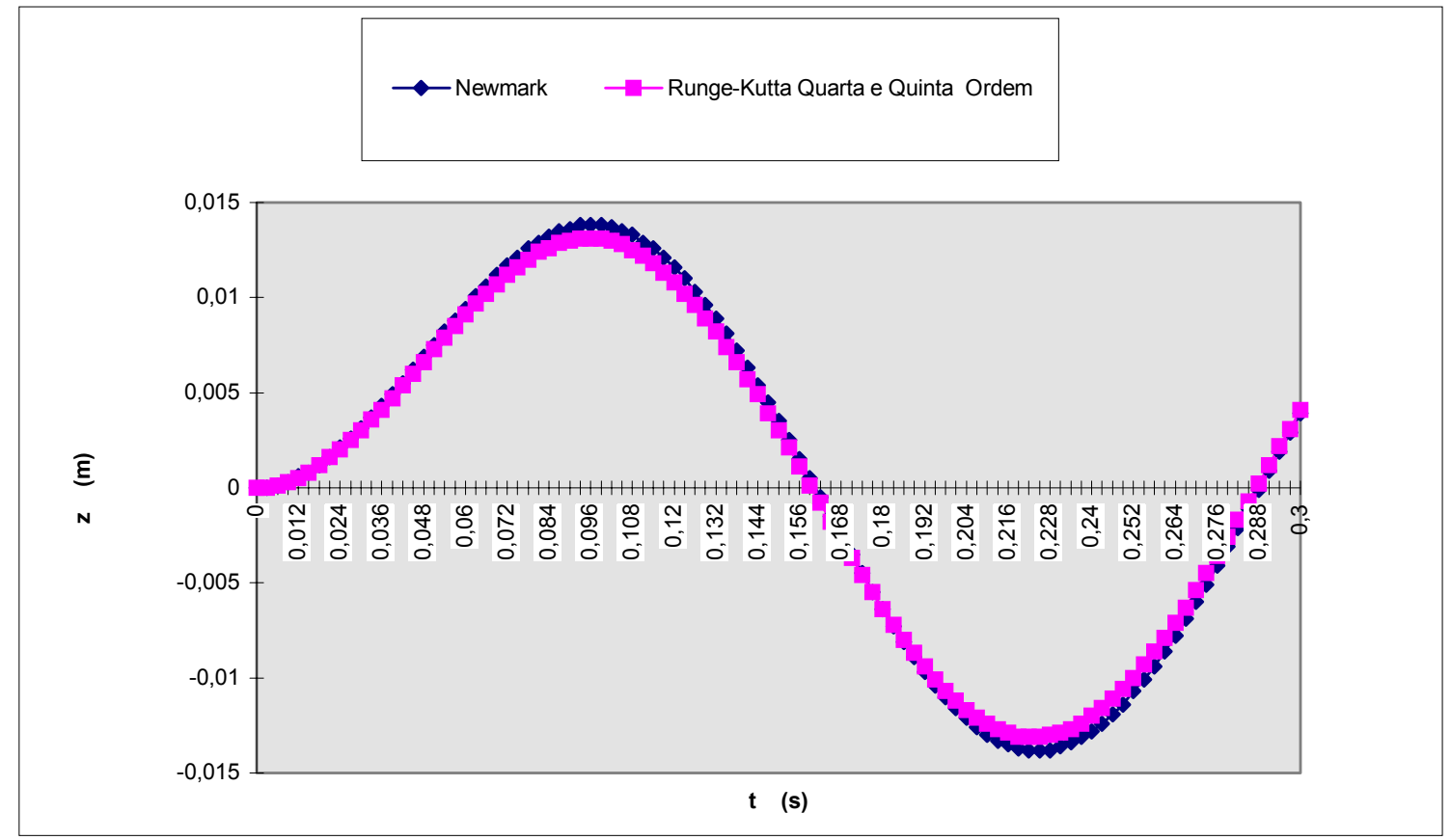

Gráfico 5.1.b - Valores do deslocamento para diferentes métodos de solução da equação do movimento.

Tabela 5.3 - Número de minimizações sem restrições para diferentes valores de $x_{0}$.

\begin{tabular}{|l|c|c|c|c|c|c|}
\cline { 2 - 7 } \multicolumn{1}{c|}{} & \multicolumn{7}{c|}{ K } \\
\cline { 2 - 7 } \multicolumn{1}{c|}{} & Newm/Splin & Newm/Trap & Rk4/Splin & Rk4/Trap & Rk5/Splin & Rk5/Trap \\
\hline CASO 1 & 13 & 10 & 10 & 13 & 10 & 10 \\
\hline CASO 2 & 27 & 20 & 26 & 20 & 16 & 20 \\
\hline
\end{tabular}

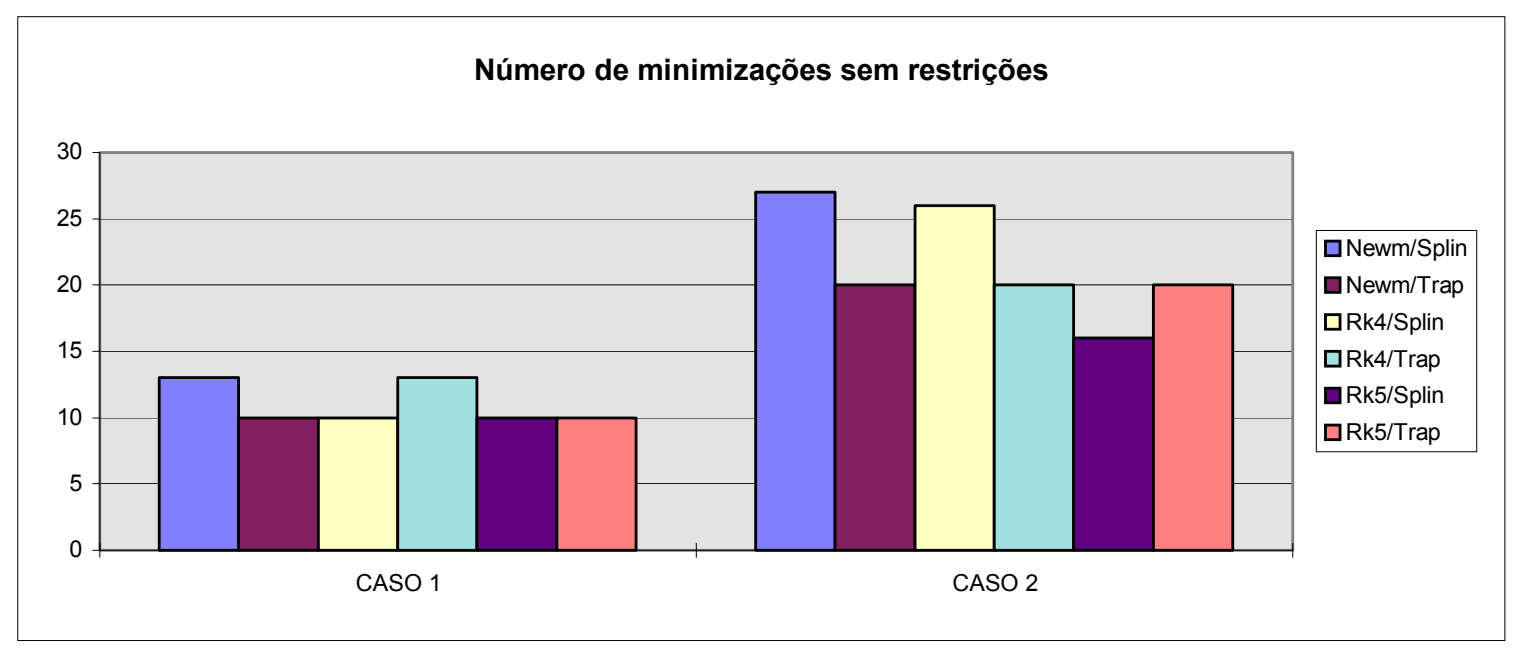

Gráfico 5.2 - Número de minimizações sem restrições para diferentes valores de $x_{\mathrm{o}}$ (tabela 5.3) 
Tabela 5.4 - Valores finais da variável de projeto para diferentes valores de $x_{0}$.

\begin{tabular}{|c|c|c|c|c|c|c|c|}
\cline { 3 - 8 } \multicolumn{1}{c|}{} & \multicolumn{6}{c|}{ Xf.1E4 } \\
\cline { 2 - 8 } \multicolumn{1}{c|}{} & Xo.1E4 & Newm/Splin & Newm/Trap & Rk4/Splin & Rk4/Trap & Rk5/Splin & Rk5/Trap \\
\hline CASO 1 & 10,000 & 15,184 & 15,184 & 4,407 & 4,407 & 4,412 & 4,412 \\
\hline CASO 2 & 0,000 & 15,184 & 15,184 & 4,407 & 4,407 & 4,412 & 4,412 \\
\hline
\end{tabular}

Tabela 5.5 - Valores finais da função objetivo para diferentes valores de $x_{0}$.

\begin{tabular}{|c|c|c|c|c|c|c|c|}
\cline { 2 - 8 } \multicolumn{1}{c|}{} & $\mathbf{f ( X o )}$ & Newm/Splin & Newm/Trap & Rk4/Splin & Rk4/Trap & Rk5/Splin & Rk5/Trap \\
\hline CASO 1 & 1114,0 & 1280,4 & 1280,4 & 847,7 & 847,7 & 848,1 & 848,1 \\
\hline CASO 2 & 0,0 & 1280,4 & 1280,4 & 847,7 & 847,7 & 848,1 & 848,1 \\
\hline
\end{tabular}

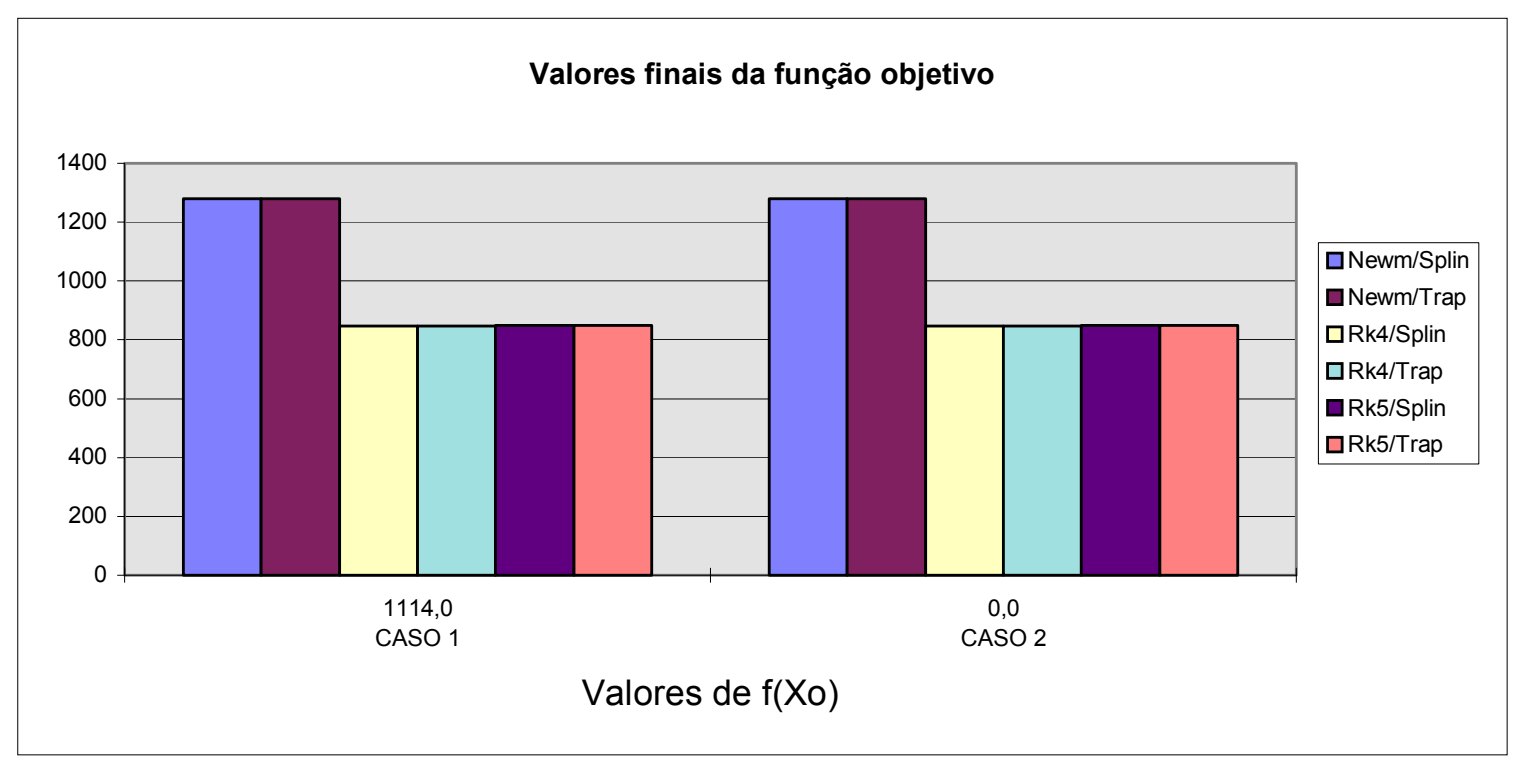

Gráfico 5.3 - Valores finais da função objetivo para diferentes valores de $x_{\mathrm{o}}$ (tabela 5.5).

Observa-se novamente a diferença no valor final da função objetivo entre os métodos de Newmark e Runge-Kutta.

Tabela 5.6 - Número de minimizações sem restrições para diferentes valores de $x_{0}$.

\begin{tabular}{|l|c|c|c|c|c|c|}
\cline { 2 - 7 } \multicolumn{1}{c|}{} & \multicolumn{7}{c|}{ K } \\
\cline { 2 - 7 } \multicolumn{1}{c|}{} & Newm/Splin & Newm/Trap & Rk4/Splin & Rk4/Trap & Rk5/Splin & Rk5/Trap \\
\hline CASO 1 & 10 & 12 & 11 & 12 & 12 & 11 \\
\hline CASO 2 & 29 & 19 & 19 & 12 & 12 & 11 \\
\hline
\end{tabular}


O gráfico 5.4 mostra que o número de minimizações sem restrições é maior no caso 2 que no caso 1 , e novamente o valor final da função objetivo permanece praticamente inalterado para os dois casos.

Já no gráfico 5.5 observa-se que os valores finais da função objetivo com os métodos Runge-Kutta são mais sensíveis à diminuição do número de subdivisões no intervalo de tempo.

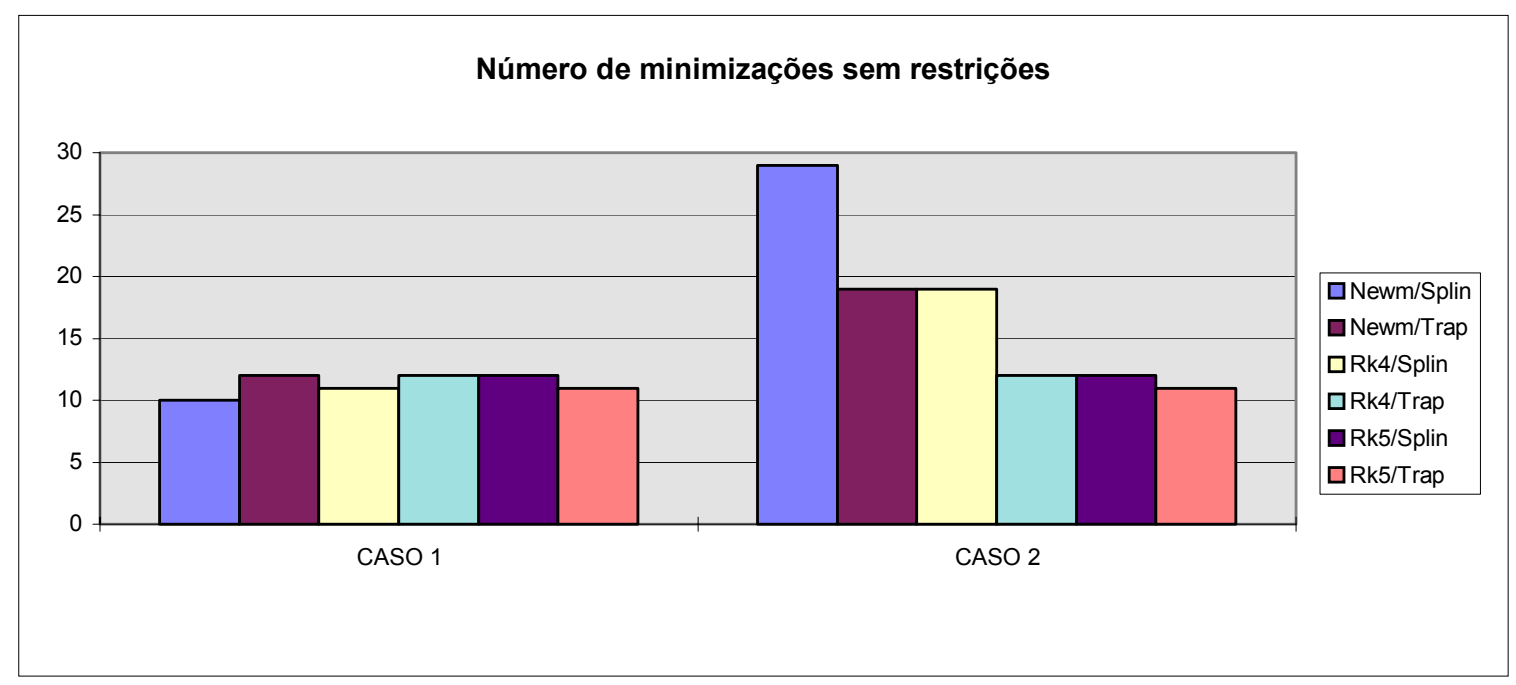

Gráfico 5.4 - Número de minimizações sem restricões para diferentes valores de $x_{\mathrm{o}}$ (tabela 5.6)

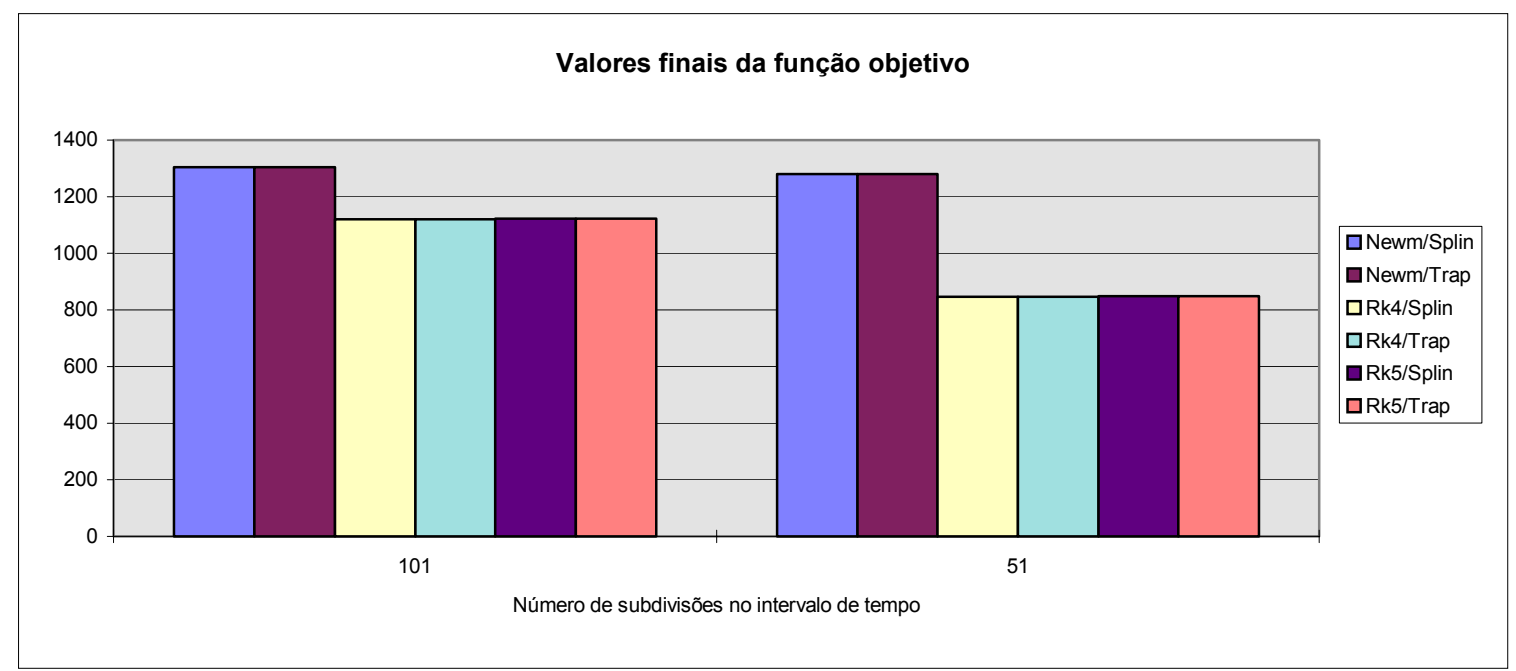

Gráfico 5.5 - Valores finais da função objetivo no caso 1 para diferentes valores de $n$. 


\subsection{EXEMPLO 2 (Goldenberg, Silva, Brasil e Pimenta[17])}

\section{Pórtico Viscoelastoplástico com um grau de liberdade:}

O pórtico analisado é o Shear-building da figura 5.1, porém, neste exemplo, com comportamento elastoplástico perfeito.

O material dos pilares é concreto com módulo de elasticidade $E=20 \mathrm{GPa}$, densidade $\rho=2,5 \mathrm{t} / \mathrm{m}^{3}$ e tensão de escoamento $\sigma_{e}=20 \mathrm{MPa}$. Considera-se um amortecimento viscoso $C$ igual a $2.5 \%$ do amortecimento crítico de uma estrutura linear equivalente $(C=10000 N s / m)$. O momento de plastificação total de uma seção retangular é $M_{p}=\left(3 \sigma_{e} / d\right) \times I$.

As condições iniciais para o deslocamento $z$ são $z(0)=0$ e $z(0)=0$. Assume-se o intervalo de tempo $[0,1]$ em segundos e subdividido em $(n-1)$ intervalos. O máximo deslocamento permitido é $z_{b}=0,04 m$ para $t \in[0,1]$. A massa $M(x)$ é igual a da viga $\mathrm{BC}, 12000 \mathrm{Kg}$, somada a 13/35 da massa dos pilares $\mathrm{AB}$ e $\mathrm{CD}$; as colunas são retangulares $b \times d \operatorname{com} b=$ $0.12 \mathrm{~m}$. A variável de projeto e a função objetivo são respectivamente o momento de inércia $x$, em $m^{4}$, e a massa dos pilares $f(x)$, em $K g$.

A rigidez dos pilares $K(x)$ em $N / m$, a área da seção transversal dos pilares em $m^{2}$, a massa $M(x)$ em $K g$, e a tensão de flexão em $N / m^{2}$ podem ser calculadas por 5.1 à 5.4.

O problema de otimização é então

minimizar

$$
f(x)=2 \times 2.5 \times 10^{3} \times 4 A(x),
$$

sujeito a:

$$
|z(t)|-z_{b} \leq 0, \quad t \in[0,1] \quad \mathrm{e}
$$

O valor mínimo para a variável $x$ para que a flexão ocorra em torno do eixo principal de inércia é $0.12 \times 0.12^{3} / 12=1.72810^{-5}$, posto isto, impõe-se a restrição

$$
-x+1.72810^{-5} \leq 0 \text {. }
$$


O valor de $z(t)$ é obtido pela integração da equação

$$
M(x) \ddot{z}(t)+C \dot{z}(t)+R(x, z(t))=p(t),
$$

para $t \in[0,1] s$ com as condições iniciais $z(0)=0$ e $z(0)=0$.

A força restauradora elastoplástica perfeita é definida por:

$$
R\left(x, z\left(t_{k}\right)\right)=\left\{\begin{array}{c}
K(x) z\left(t_{k}\right) \text { se }\left|K(x) z\left(t_{k}\right)\right|<A(x) \times 10^{7} \\
\operatorname{sign} \times A(x) \times 10^{7} \text { se }\left|K(x) z\left(t_{k}\right)\right| \geq A(x) \times 10^{7},
\end{array}\right.
$$

onde sign é função dos sinais das diferenças $\left(z_{k}-z_{k-1}\right)$ e $\left(\dot{z}_{k}-\dot{z}_{k-1}\right)$ conforme descrito em [11].

Analogamente ao exemplo 1, este exemplo foi calculado para dois valores iniciais das variáveis de projeto. Um valor inicial de $x$ contido na região viável (caso 1), e outro valor inicial fora da região viável (caso 2).

O número de 100 subdivisões nas tabelas 5.7 à 5.9 e de 50 subdivisões nas tabelas 5.10 à 5.12 foi adotado na discretização do intervalo de tempo $[0,1] \mathrm{s}$. Os resultados obtidos vêm a seguir.

Tabela 5.7 - Valores finais das variáveis de projeto para diferentes valores de $x_{0}$.

\begin{tabular}{|l|c|c|c|c|c|}
\cline { 3 - 6 } \multicolumn{1}{c|}{} & \multicolumn{4}{c|}{ Xf.1E4 } \\
\cline { 2 - 6 } \multicolumn{1}{c|}{} & Xo. 1E4 & Rk4/Splin & Rk4/Trap & Rk5/Splin & Rk5/Trap \\
\hline CASO 1 & 10,000 & 0,472 & 0,477 & 0,297 & 0,297 \\
\hline CASO 2 & 0,000 & 0,472 & 0,477 & 0,297 & 0,297 \\
\hline
\end{tabular}

Tabela 5.8 - Valores finais da função objetivo para diferentes valores de $x_{\mathrm{o}}$.

\begin{tabular}{|l|c|c|c|c|c|}
\cline { 3 - 6 } \multicolumn{1}{c|}{} & $\mathbf{f ( X o )}$ & Rk4/Splin & Rk4/Trap & Rk5/Splin & Rk5/Trap \\
\cline { 2 - 6 } \multicolumn{1}{c|}{} & 1114,0 & 403,2 & 403,9 & 345,1 & 345,1 \\
\hline CASO 1 & 0,0 & 403,2 & 403,9 & 345,0 & 345,0 \\
\hline
\end{tabular}

Observa-se no gráfico 5.6 que os valores da função objetivo apresentados pelo método de Runge-Kutta de quinta ordem foram melhores que os apresentados pelo de quarta ordem. 


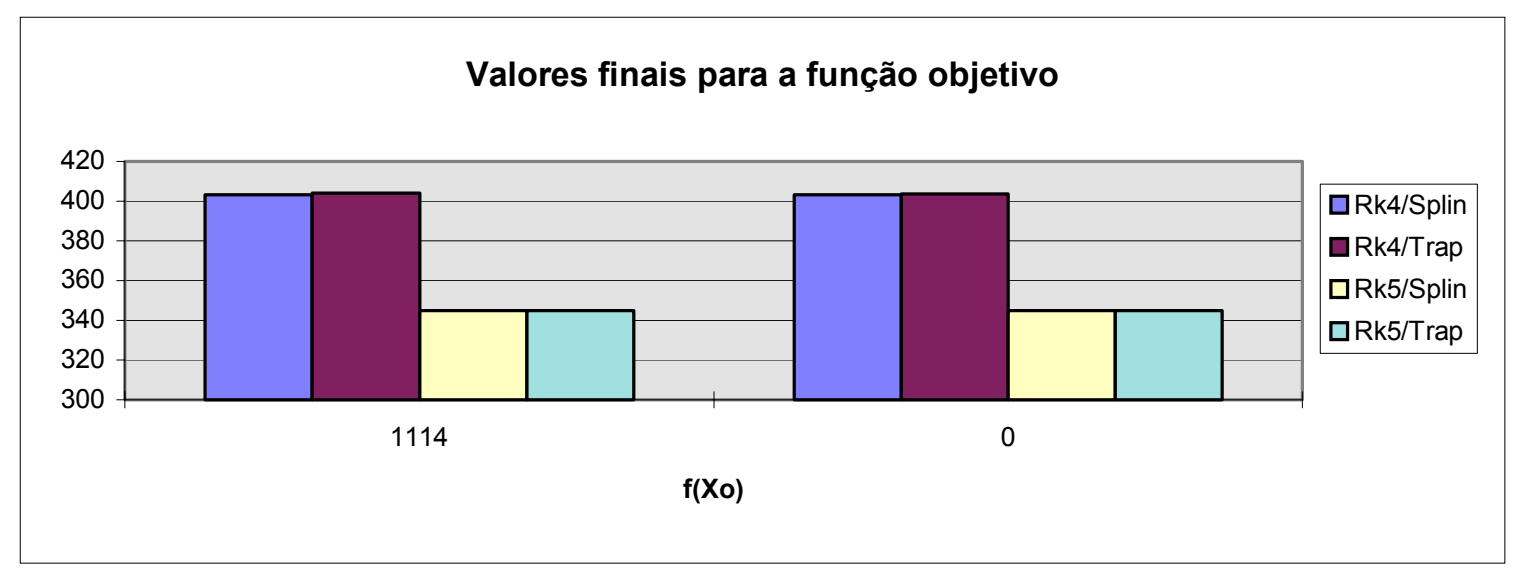

Gráfico 5.6 - Valores finais da função objetivo para diferentes valores de $x_{0}$. (tabela 5.8)

Tabela 5.9 - Número de minimizações sem restrições para diferentes valores de $x_{0}$.

\begin{tabular}{|l|c|c|c|c|}
\cline { 2 - 5 } \multicolumn{1}{c|}{} & \multicolumn{4}{c|}{$\mathbf{K}$} \\
\cline { 2 - 5 } \multicolumn{1}{c|}{} & Rk4/Splin & Rk4/Trap & Rk5/Splin & Rk5/Trap \\
\hline CASO 1 & 21 & 22 & 20 & 20 \\
\hline CASO 2 & 22 & 22 & 20 & 20 \\
\hline
\end{tabular}

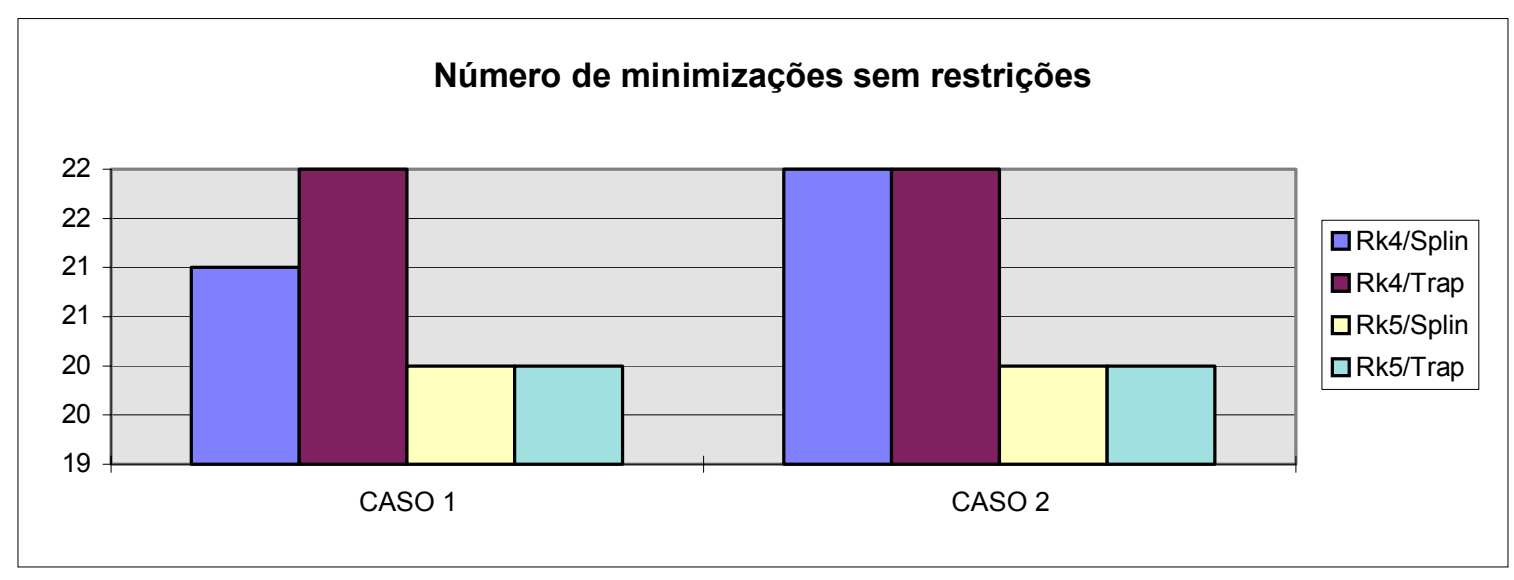

Gráfico 5.7 - Número de minimizações sem restrições para diferentes valores de $x_{0}$ (tabela 5.9).

No gráfico acima verifica-se que o número de minimizações sem restrições apresentadas pelos métodos Runge-Kutta de quinta ordem são inferiores aos apresentados pelo de quarta ordem. Quanto à utilização de splines ou trapézio os resultados dos gráficos 5.6 e 5.7 mostram que não há diferenças significativas nos valores apresentados. 
Tabela 5.10 - Valores finais das variáveis de projeto para diferentes valores de $x_{0}$.

\begin{tabular}{|l|c|c|c|c|c|}
\cline { 3 - 6 } \multicolumn{1}{c|}{} & \multicolumn{4}{c|}{ Xf.1E10 } \\
\cline { 2 - 6 } \multicolumn{1}{c|}{} & Xo.1E10 & Rk4/Splin & Rk4/Trap & Rk5/Splin & Rk5/Trap \\
\hline CASO 1 & 10,000 & 0,292 & 0,293 & 0,173 & 0,173 \\
\hline CASO 2 & 0,000 & 0,293 & 0,293 & 0,173 & 0,173 \\
\hline
\end{tabular}

Tabela 5.11 - Valores finais da função objetivo para diferentes valores de $x_{0}$.

\begin{tabular}{|l|c|c|c|c|c|}
\cline { 3 - 6 } \multicolumn{1}{c|}{} & $\mathbf{f ( X o )}$ & Rk4/Splin & Rk4/Trap & Rk5/Splin & Rk5/Trap \\
\hline CASO 1 & 1114,0 & 343,1 & 343,6 & 288,0 & 288,0 \\
\hline CASO 2 & 0,0 & 343,3 & 343,6 & 288,0 & 288,0 \\
\hline
\end{tabular}

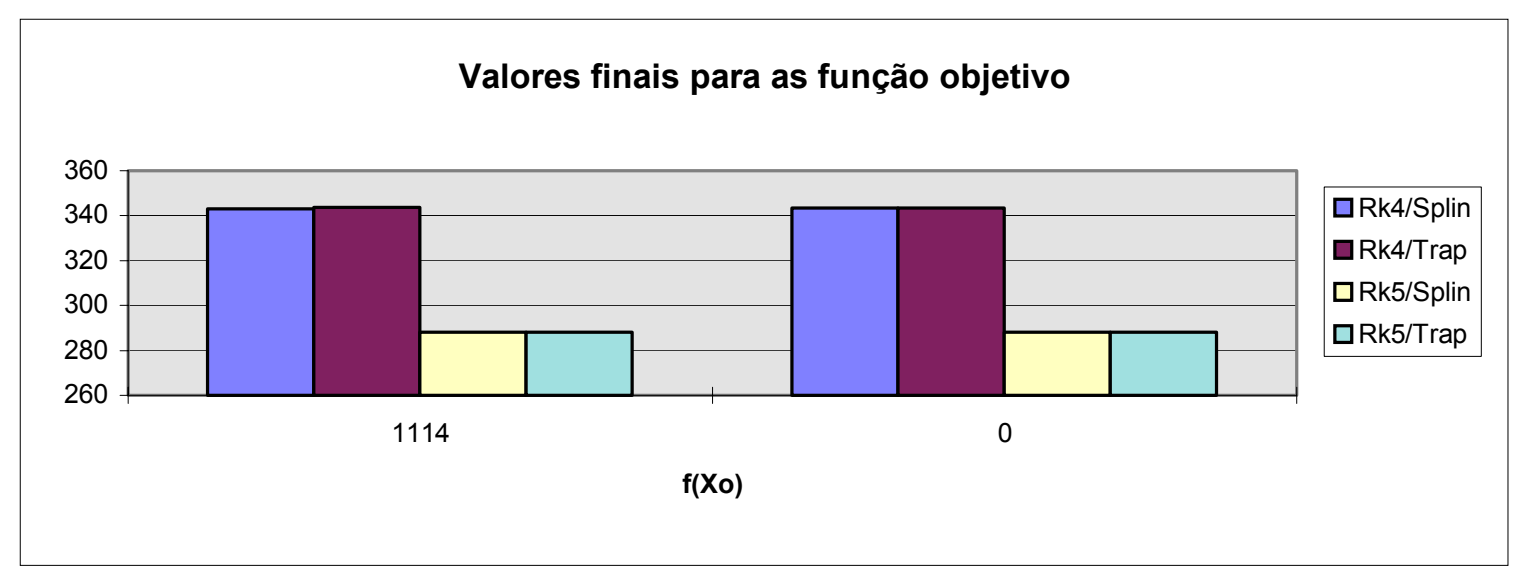

Gráfico 5.8 - Valores da função objetivo para diferentes valores de $x_{\mathrm{o}}$ (tabela 5.11).

Observa-se nos gráficos 5.6 e 5.8 que o valor inicial de $x$ pouco interfere no valor final da função objetivo.

Tabela 5.12 - Número de minimizações sem restrições para diferentes valores de $x_{0}$.

\begin{tabular}{|l|c|c|c|c|}
\cline { 2 - 5 } \multicolumn{1}{c|}{} & \multicolumn{4}{c|}{ K } \\
\cline { 2 - 5 } \multicolumn{1}{c|}{} & Rk4/Splin & Rk4/Trap & Rk5/Splin & Rk5/Trap \\
\hline CASO 1 & 20 & 22 & 15 & 15 \\
\hline CASO 2 & 19 & 20 & 15 & 15 \\
\hline
\end{tabular}

Novamente se observa que o número de minimizações sem restrições apresentadas pelos métodos Runge-Kutta de quinta ordem são inferiores aos apresentados pelo de quarta ordem, e também que a utilização de splines ou trapézio é praticamente indiferente no desempenho das diversas combinações dos métodos. 


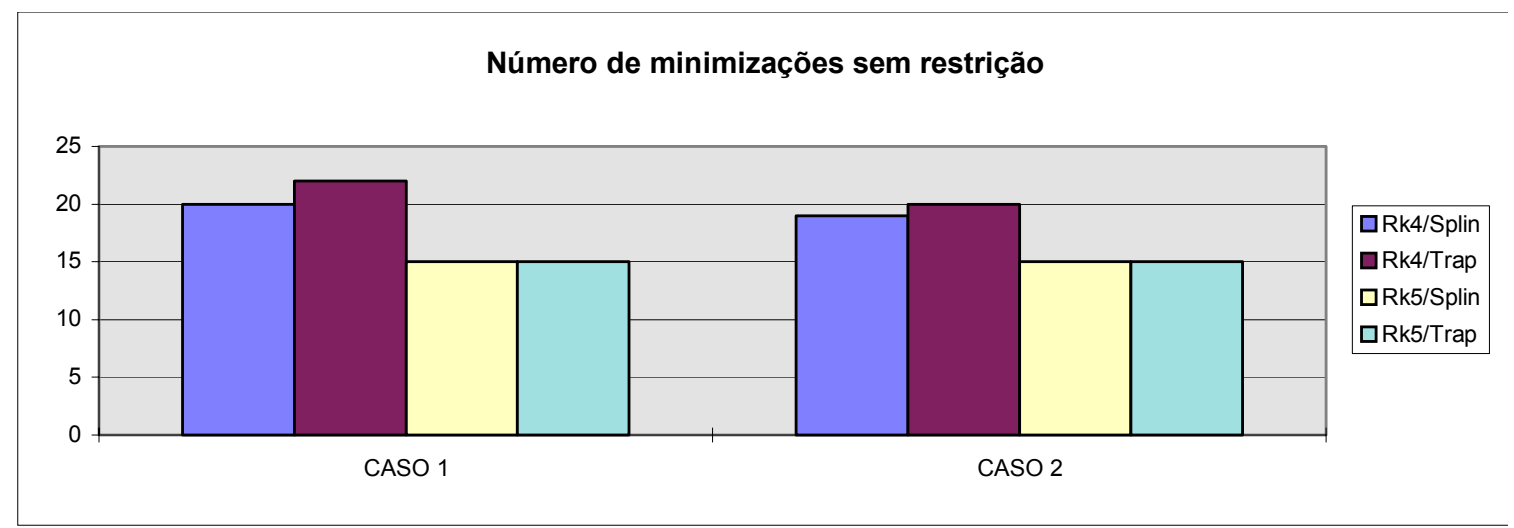

Gráfico 5.9 - Número de minimizações sem restrições para diferentes valores de $x_{\mathrm{o}}$ (tabela 5.12)

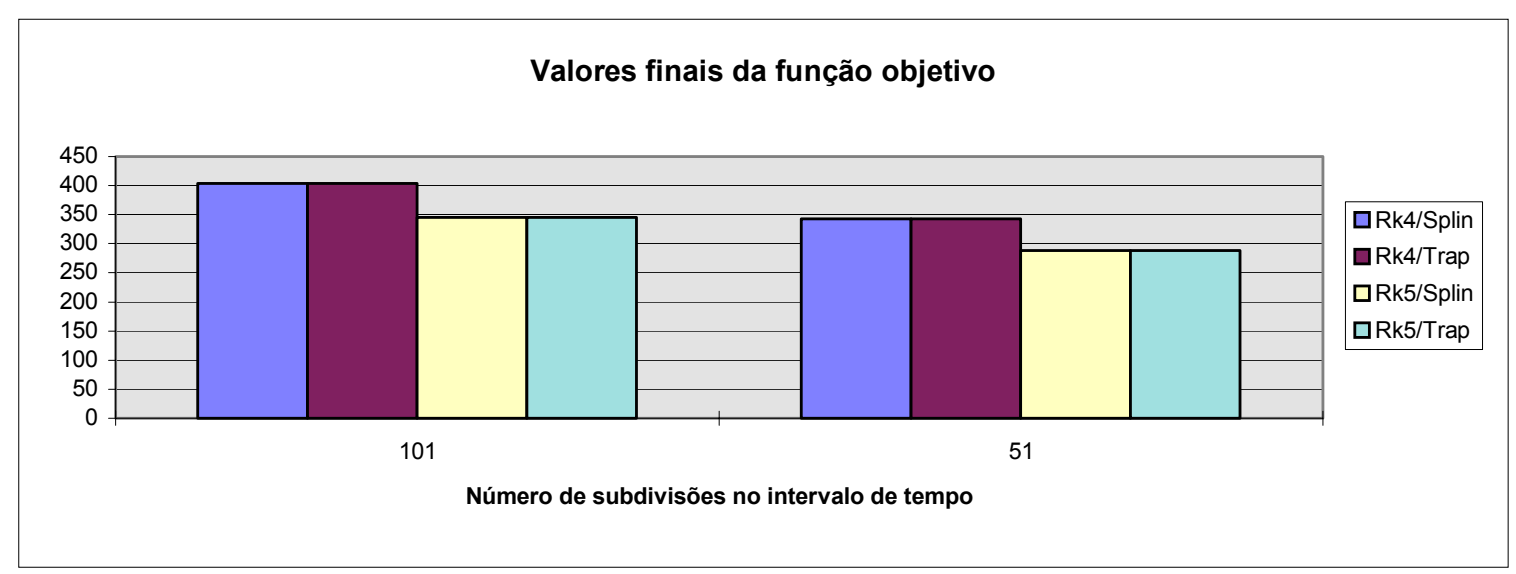

Gráfico 5.10 - Valores finais da função objetivo no caso 1 para diferentes valores de $n$.

Observa-se no gráfico acima uma grande sensibilidade dos métodos Runge-Kutta à diminuição do número de subdivisões no intervalo de tempo. Este resultado nos mostra novamente que o método do Lagrangeano Aumentado é bastante sensível à solução da equação do movimento. 


\subsection{EXEMPLO 3}

\section{Um absorvedor de impacto linear:}

Imaginem um navio se aproximando do porto, conforme figura 5.2. Certamente ele irá se chocar com a parede do porto ao atracar. É então necessário a colocação de um dispositivo para absorver seu impacto. $\mathrm{O}$ absorvedor de impacto minimiza a aceleração no choque $\mathrm{e}$ evita danos tanto à estrutura do ancoradouro quanto à do navio.

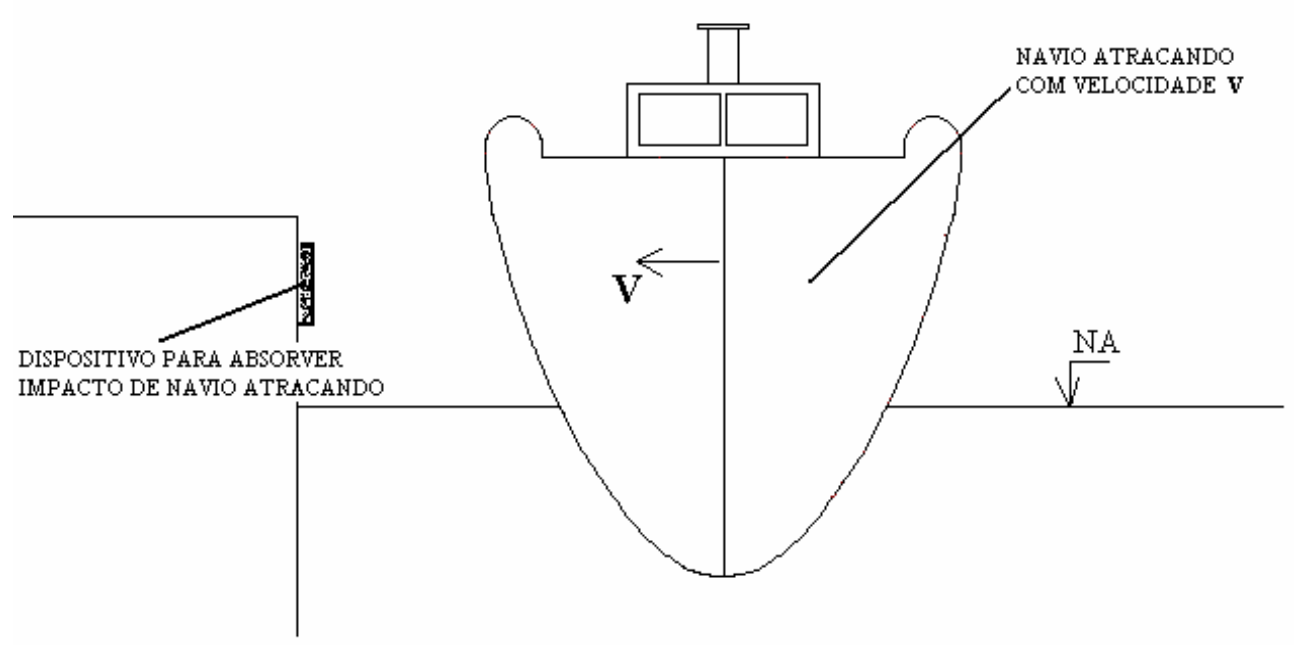

Figura 5.2 - Exemplo de aplicação de absorvedor de impacto

Um modelo matemático de absorvedor de impacto linear de massa $M$ fixada é indicado na figura 5.3. São adotadas duas variáveis de projeto $x_{1}$ e $x_{2}$, que representam respectivamente o coeficiente elástico e do amortecimento viscoso.

O impacto é representado por uma velocidade inicial $v$ e a equação do movimento é então

$$
M \ddot{z}(t)+x_{2} \dot{z}(t)+x_{1} z(t)=0, \quad t \in[0,12] .
$$

As condições iniciais são $z(0)=0$ e $z(0)=v$. 
O objetivo consiste em minimizar a máxima aceleração da massa e para tanto é criada uma variável artificial de projeto $x_{3}$ que deve satisfazer

$$
\frac{1}{M}\left|x_{2} z(t)+x_{1} z(t)\right|-x_{3} \leq 0, \quad t \in[0,12]
$$

e a função objetivo é

$$
f(x)=x_{3} .
$$

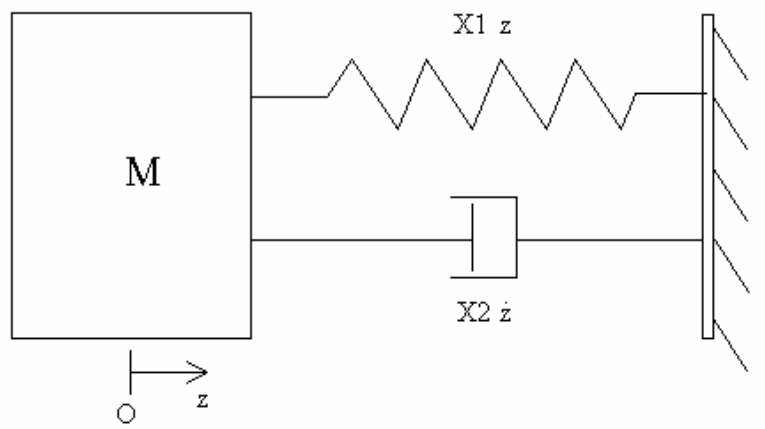

Figura 5.3 - Modelo matemático de um absorvedor de impácto linear

Para se evitar um valor alto para a aceleração no impacto impõe-se

$$
\left(\frac{x_{2} v}{M}\right)-x_{3} \leq 0
$$

Finalmente, o deslocamento máximo deve ser restrito à

$$
|z(t)|-z_{b} \leq 0, \quad t \in[0,12] .
$$

Outros dados numéricos adotados são $M=1, v=1$ e $z_{b}=1$.

Este exemplo foi resolvido para dois valores iniciais das variáveis de projeto, os casos 1 e 2. Um número de 80 subdivisões nas tabelas 5.13 à 5.15 e 40 subdivisões nas tabelas 5.16 à 5.18 foram adotadas na discretização do intervalo de tempo $[0,12] s$. 
Tabela 5.13 - Valores finais das variáveis de projeto para diferentes valores de $\boldsymbol{x}_{0}$.

\begin{tabular}{|c|c|c|c|c|c|c|c|c|}
\cline { 4 - 9 } \multicolumn{2}{c|}{} & \multicolumn{8}{c|}{ Xf } \\
\cline { 3 - 9 } \multicolumn{2}{c|}{} & $\mathbf{X o}$ & Newm/Splin & Newm/Trap & Rk4/Splin & Rk4/Trap & Rk5/Splin & Rk5/Trap \\
\hline \multirow{3}{*}{ CASO 1 } & $\mathbf{X 1}$ & 0,300 & 0,375 & 0,374 & 0,375 & 0,374 & 0,375 & 0,374 \\
\cline { 2 - 9 } & $\mathbf{X}$ & 0,200 & 0,471 & 0,473 & 0,470 & 0,472 & 0,470 & 0,472 \\
\hline & $\mathbf{X}$ & 0,300 & 0,542 & 0,521 & 0,541 & 0,521 & 0,541 & 0,523 \\
\hline \multirow{3}{*}{ CASO 2 2} & $\mathbf{X 1}$ & 0,400 & 0,378 & 0,379 & 0,375 & 0,376 & 0,375 & 0,376 \\
\cline { 2 - 9 } & $\mathbf{X 2}$ & 0,100 & 0,468 & 0,468 & 0,470 & 0,470 & 0,470 & 0,470 \\
\cline { 2 - 9 } & $\mathbf{X 3}$ & 0,400 & 0,521 & 0,522 & 0,522 & 0,521 & 0,522 & 0,521 \\
\hline
\end{tabular}

Tabela 5.14 - Valores finais da função objetivo para diferentes valores de $\boldsymbol{x}_{0}$.

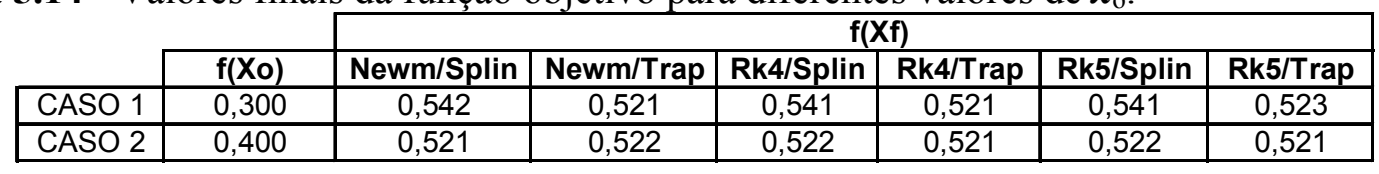

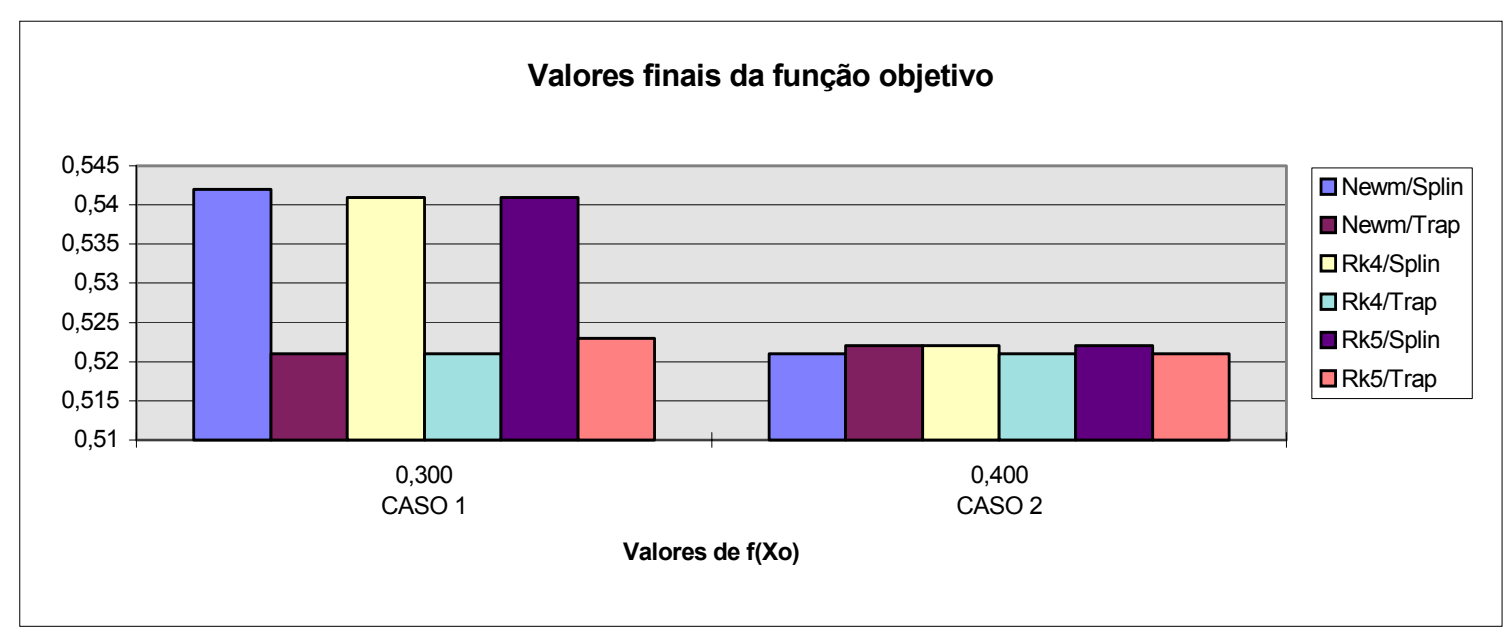

Gráfico 5.11 - Valores finais da função objetivo para diferentes valores de $\boldsymbol{x}_{\mathrm{o}}$ (tabela 5.14).

Observa-se no gráfico acima que para o caso 1, o Lagrangeano Aumentado apresenta melhores valores com o utilização da regra do trapézio para integração das violações de restrições. Na maioria dos casos o valor inicial das variáveis de projeto pouco interferem no valor final de $f(\boldsymbol{x})$.

Tabela 5.15 - Número de minimizações sem restrições para diferentes valores de $\boldsymbol{x}_{0}$.

\begin{tabular}{|l|c|c|c|c|c|c|}
\cline { 2 - 7 } \multicolumn{1}{c|}{} & \multicolumn{7}{c|}{ K } \\
\cline { 2 - 7 } \multicolumn{1}{c|}{} & Newm/Splin & Newm/Trap & Rk4/Splin & Rk4/Trap & Rk5/Splin & Rk5/Trap \\
\hline CASO 1 & 12 & 10 & 12 & 10 & 12 & 10 \\
\hline CASO 2 & 10 & 10 & 18 & 10 & 16 & 10 \\
\hline
\end{tabular}




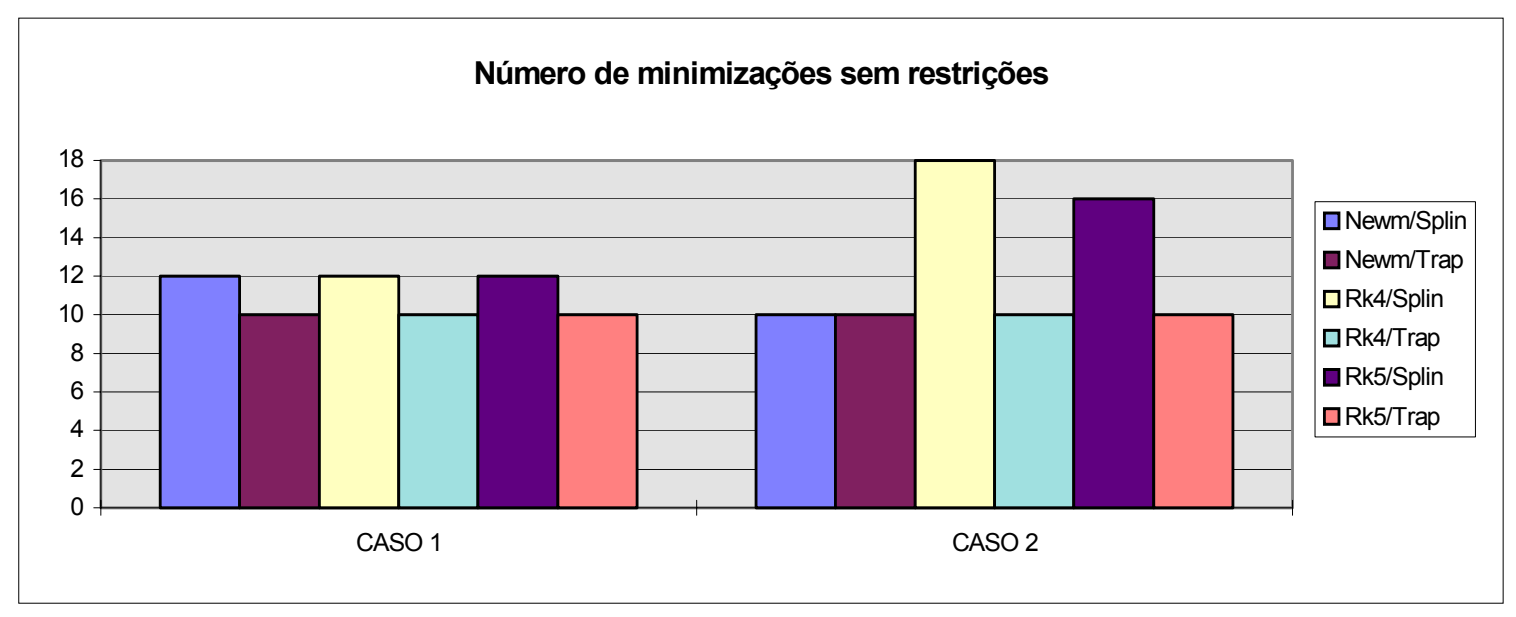

Gráfico 5.12 - Número de minimizações sem restrições para diferentes valores de $\boldsymbol{x}_{\mathrm{o}}$ (tabela 5.15)

Observa-se novamente no gráfico acima que a solução com a regra do trapézio apresenta um melhor desempenho do que com splines cúbicos.

Tabela 5.16 - Valores finais das variáveis de projeto para diferentes valores de $\boldsymbol{x}_{0}$.

\begin{tabular}{|c|c|c|c|c|c|c|c|c|}
\cline { 4 - 9 } \multicolumn{2}{c|}{} & \multicolumn{8}{c|}{ Xf } \\
\cline { 3 - 9 } \multicolumn{2}{c|}{} & $\mathbf{X o}$ & Newm/Splin & Newm/Trap & Rk4/Splin & Rk4/Trap & Rk5/Splin & Rk5/Trap \\
\hline \multirow{3}{*}{ CASO 1 } & $\mathbf{X 1}$ & 0,300 & 0,399 & 0,376 & 0,409 & 0,376 & 0,408 & 0,376 \\
\cline { 2 - 9 } & $\mathbf{X 2}$ & 0,200 & 0,459 & 0,474 & 0,449 & 0,471 & 0,448 & 0,471 \\
\hline & $\mathbf{X 3}$ & 0,300 & 0,527 & 0,523 & 0,529 & 0,521 & 0,529 & 0,521 \\
\hline \multirow{3}{*}{ CASO 2 } & $\mathbf{X 1}$ & 0,400 & 0,409 & 0,376 & 0,565 & 0,376 & 0,517 & 0,376 \\
\cline { 2 - 9 } & $\mathbf{X 2}$ & 0,100 & 0,472 & 0,474 & 0,509 & 0,470 & 0,341 & 0,470 \\
\cline { 2 - 9 } & $\mathbf{X 3}$ & 0,400 & 0,535 & 0,523 & 0,616 & 0,521 & 0,649 & 0,521 \\
\hline
\end{tabular}

Tabela 5.17 - Valores finais da função objetivo diferentes valores de $\boldsymbol{x}_{0}$.

\begin{tabular}{|c|c|c|c|c|c|c|c|}
\cline { 2 - 8 } \multicolumn{1}{c|}{} & $\mathbf{f}(\mathbf{X o})$ & Newm/Splin & Newm/Trap & Rk4/Splin & Rk4/Trap & Rk5/Splin & Rk5/Trap \\
\hline CASO 1 & 0,300 & 0,527 & 0,523 & 0,529 & 0,521 & 0,529 & 0,521 \\
\hline CASO 2 & 0,400 & 0,535 & 0,523 & 0,616 & 0,521 & 0,649 & 0,521 \\
\hline
\end{tabular}

Tabela 5.18 - Número de minimizações sem restrições para diferentes valores de $\boldsymbol{x}_{0}$.

\begin{tabular}{|l|c|c|c|c|c|c|}
\cline { 2 - 7 } \multicolumn{1}{c|}{} & \multicolumn{7}{c|}{ K } \\
\cline { 2 - 7 } \multicolumn{1}{c|}{} & Newm/Splin & Newm/Trap & Rk4/Splin & Rk4/Trap & Rk5/Splin & Rk5/Trap \\
\hline CASO 1 & 10 & 10 & 11 & 10 & 20 & 10 \\
\hline CASO 2 & 11 & 10 & 11 & 10 & 23 & 10 \\
\hline
\end{tabular}


Observa-se no gráfico 5.13, principalmente no caso 2, que novamente a solução com regra do trapézio apresenta melhores valores que com splines cúbicos, e que o valor inicial de $\boldsymbol{x}$ pouco interferiu no valor final da função objetivo.

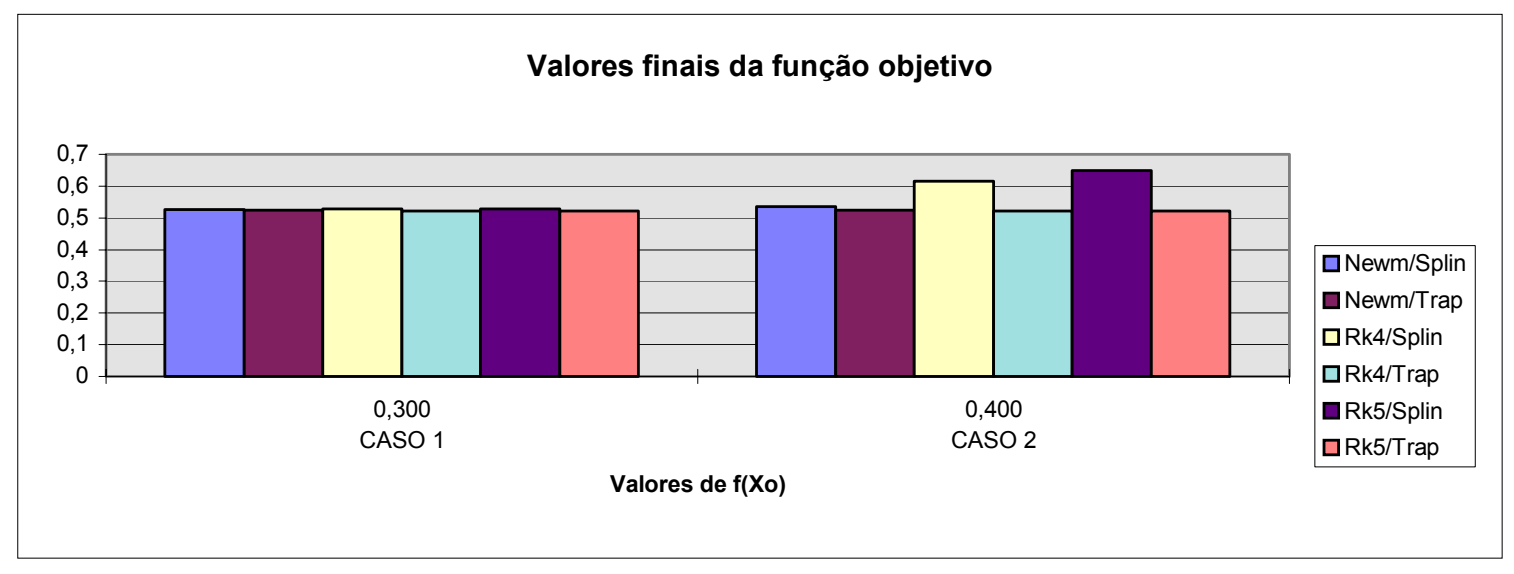

Gráfico 5.13 - Valores finais da função objetivo para diferentes valores de $\boldsymbol{x}_{\mathrm{o}}$ (tabela 5.17).

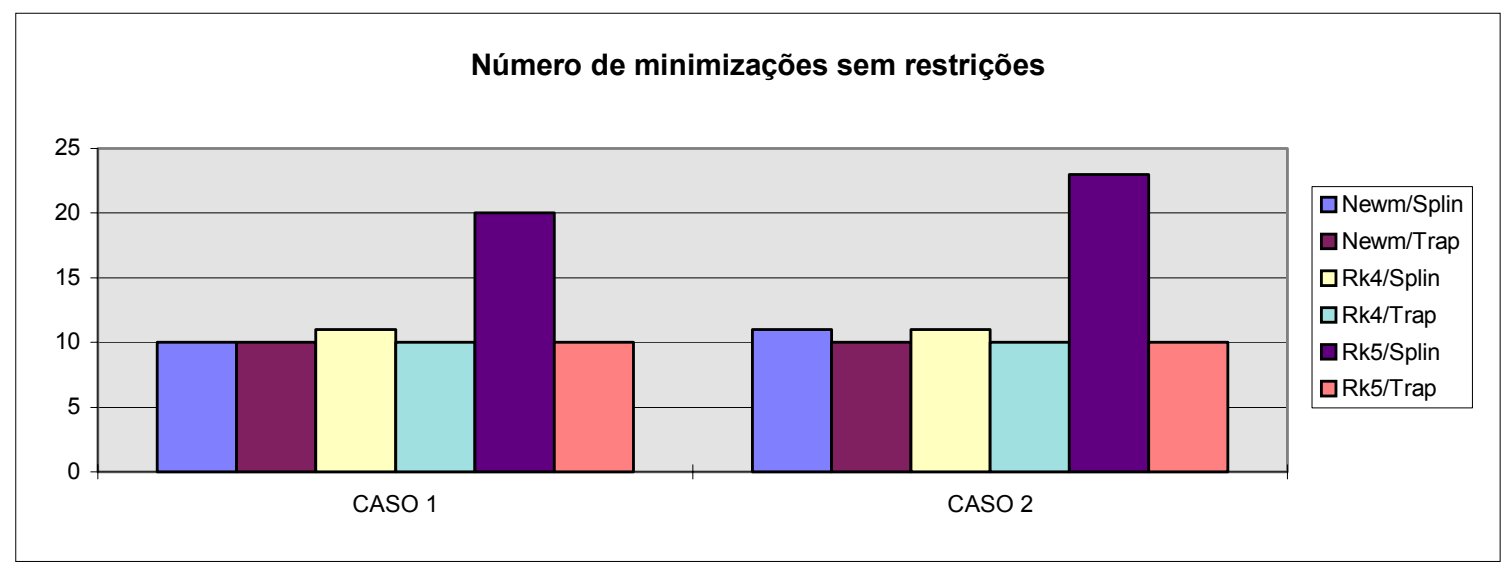

Gráfico 5.14 - Número de minimizações sem restrições para diferentes valores de $\boldsymbol{x}_{\mathrm{o}}$ (tabela 5.18).

O gráfico acima mostra que o número de minimizações sem restrições com a regra do trapézio é menor do que com splines cúbicos.

E finalmente o gráfico 5.15 mostra novamente melhores resultados com a regra do trapézio do que com splines cúbicos. 


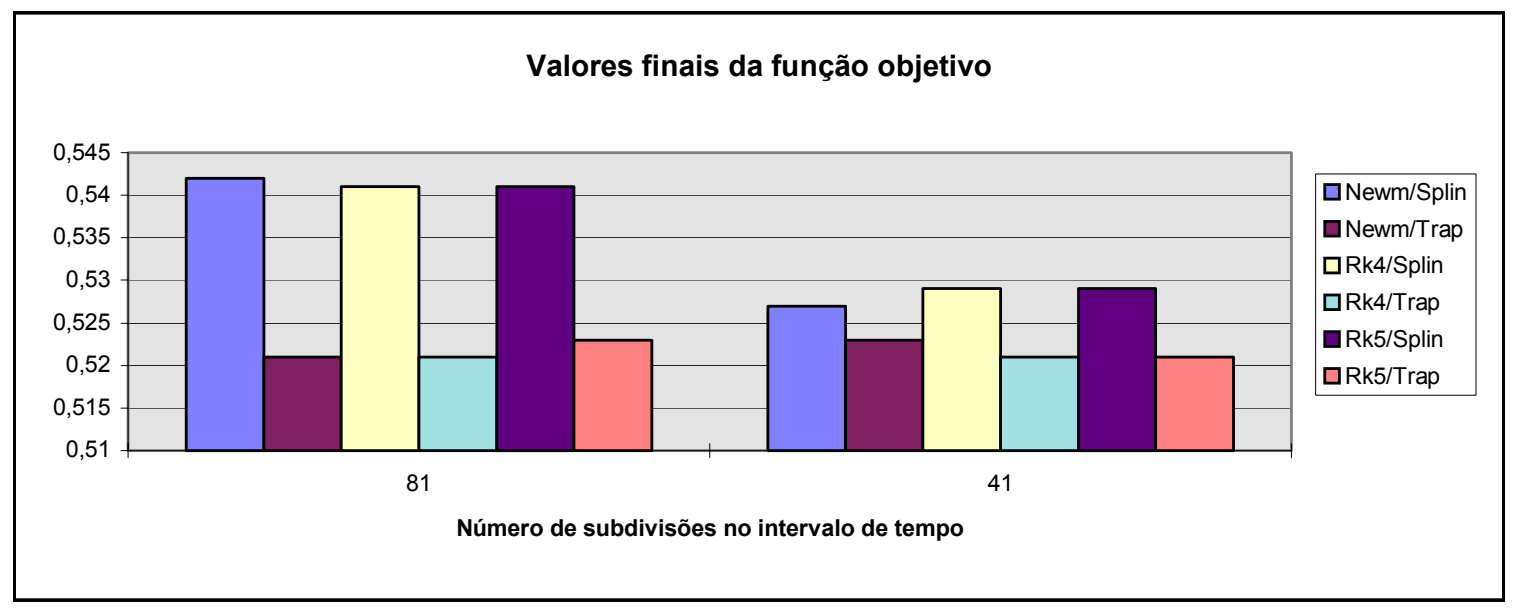

Gráfico 5.15 - Valores finais da função objetivo no caso 1 para diferentes valores de $n$. 


\subsection{EXEMPLO 4 (Haug e Arora [7])}

\section{Um absorvedor de impacto não-linear:}

O absorvedor de impacto, indicado na figura 5.4, possui massa $M$ fixada e duas variáveis de projeto $x_{1}$ e $x_{2}$, que representam respectivamente o coeficiente elástico e o amortecimento viscoso.

O impacto é representado por uma velocidade inicial $v$ e a equação do movimento é

$$
M \ddot{z}(t)+x_{2}|\dot{z}(t)|^{\omega} \operatorname{sgn}(\dot{z}(t))+x_{1}|z(t)|^{\lambda} \operatorname{sgn}(z(t))=0, \quad t \in[0,6],
$$

com as condições iniciais são $z(0)=0 \mathrm{e} \dot{z}(0)=v$.

O objetivo consiste em minimizar a máxima aceleração da massa e para tanto é criada uma variável artificial de projeto $x_{3}$ que deve satisfazer

$$
\frac{1}{M}\left|\left\{x_{2}|\dot{z}(t)|^{\omega} \operatorname{sgn}(\dot{z}(t))+x_{1}|z(t)|^{\lambda} \operatorname{sgn}(z(t))\right\}\right|-x_{3} \leq 0, \quad t \in[0,6]
$$

e a função objetivo é

$$
f(x)=x_{3}
$$

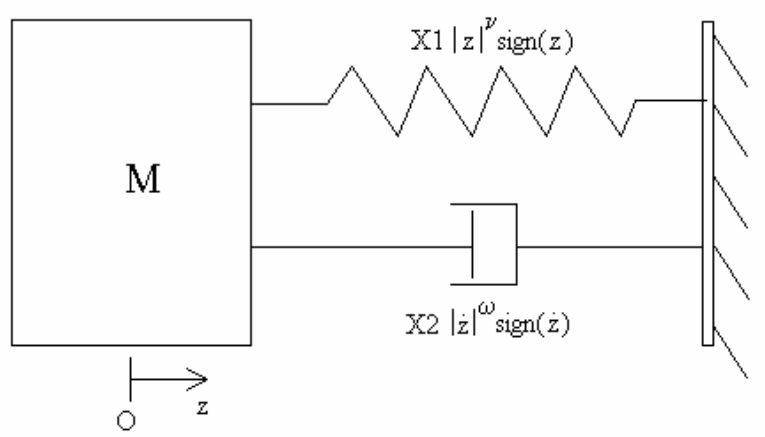

Figura 5.4 - Absorvedor de impacto não-linear 
Para se evitar um valor alto para a aceleração no impacto impõe-se

$$
\left(\frac{x_{2} v^{\omega}}{M}\right)-x_{3} \leq 0
$$

Finalmente, o deslocamento máximo deve ser restrito a

$$
|z(t)|-z_{b} \leq 0, \quad t \in[0,6] .
$$

Outros dados numéricos adotados são $M=1, v=1$ e $z_{b}=1$. O problema foi resolvido para $\lambda=2$ e $\omega=1,2,3$ e 4 .

No caso de $\omega=1$, o exemplo foi resolvido para dois valores iniciais das variáveis de projeto, os casos 1 e 2 . Um número de 40 subdivisões nas tabelas 5.19 à 5.21 e 20 subdivisões nas tabelas 5.22 à 5.24 foram adotadas na discretização do intervalo de tempo $[0,6] s$.

Resultados para $\omega=1$

Tabela 5.19 - Valores finais das variáveis de projeto para diferentes valores de $\boldsymbol{x}_{0}$.

\begin{tabular}{|c|c|c|c|c|c|c|}
\cline { 4 - 7 } \multicolumn{2}{c}{} & \multicolumn{4}{c|}{ Xf } \\
\cline { 3 - 7 } \multicolumn{2}{c|}{} & $\mathbf{X o}$ & Rk4/Splin & Rk4/Trap & Rk5/Splin & Rk5/Trap \\
\hline \multirow{3}{*}{ CASO 1 } & $\mathbf{X 1}$ & 0,500 & 0,604 & 0,441 & 0,417 & 0,441 \\
\cline { 2 - 7 } & $\mathbf{X 2}$ & 0,500 & 0,769 & 0,526 & 0,540 & 0,526 \\
\cline { 2 - 7 } & $\mathbf{X 3}$ & 0,400 & 0,768 & 0,526 & 0,573 & 0,526 \\
\hline \multirow{3}{*}{ CASO 2 } & $\mathbf{X 1}$ & 0,300 & 0,500 & 0,375 & 0,500 & 0,439 \\
\cline { 2 - 7 } & $\mathbf{X 2}$ & 0,200 & 0,532 & 0,471 & 0,531 & 0,527 \\
\cline { 2 - 7 } & $\mathbf{X 3}$ & 0,300 & 0,768 & 0,526 & 0,573 & 0,526 \\
\hline
\end{tabular}

Tabela 5.20 - Valores finais da função objetivo para diferentes valores de $\boldsymbol{x}_{0}$.

\begin{tabular}{|c|c|c|c|c|c|}
\cline { 3 - 6 } \multicolumn{1}{c|}{} & \multicolumn{4}{c|}{$\mathbf{f ( X f )}$} \\
\cline { 2 - 6 } \multicolumn{1}{c|}{} & $\mathbf{f ( X )}$ & Rk4/Splin & Rk4/Trap & Rk5/Splin & Rk5/Trap \\
\hline CASO 1 & 0,400 & 0,768 & 0,526 & 0,573 & 0,526 \\
\hline CASO 2 & 0,300 & 0,768 & 0,526 & 0,573 & 0,526 \\
\hline
\end{tabular}

Observa-se no gráfico abaixo que o valor inicial de $\boldsymbol{x}$ pouco interfere no valor final de $f(\boldsymbol{x})$. Os valores da função objetivo apresentados pelo Lagrangeano Aumentado calculado com a regra do trapézio mostra-se mais eficiente que os calculados com splines cúbicos. 


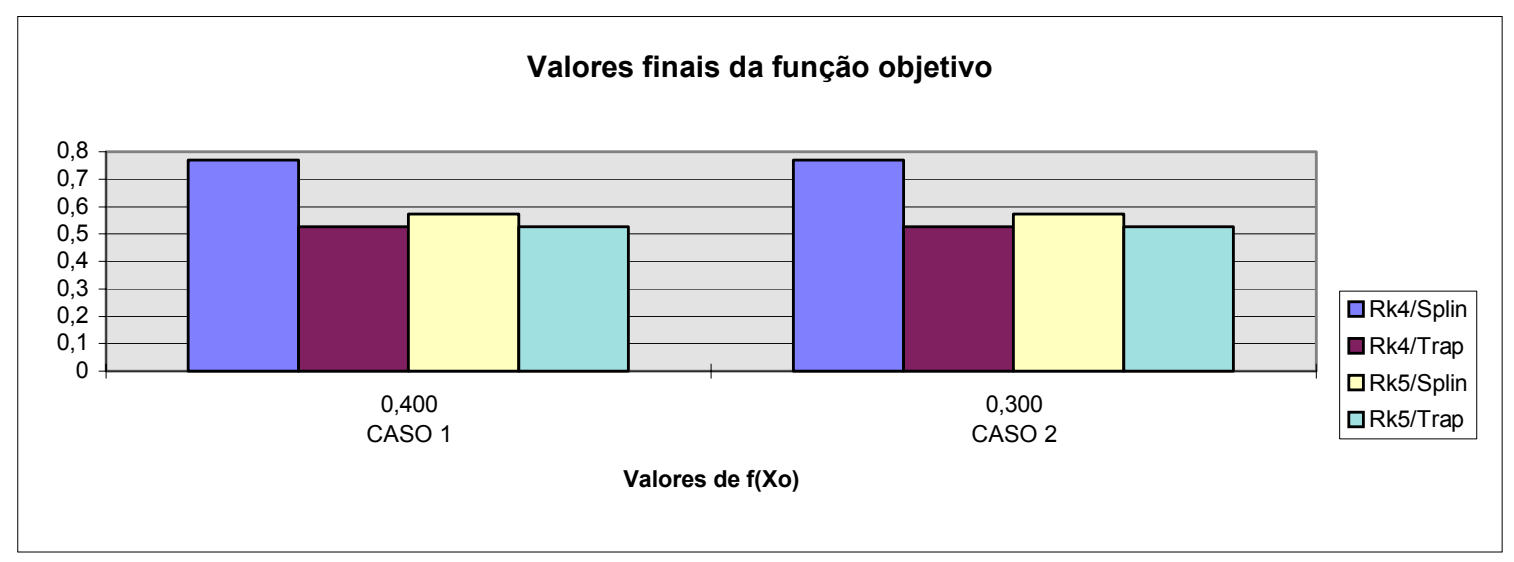

Gráfico 5.16 - Valores finais da função objetivo para diferentes valores de $\boldsymbol{x}_{\mathrm{o}}$ (tabela 5.20).

Tabela 5.21 - Número de minimizações sem restrições para diferentes valores de $\boldsymbol{x}_{0}$.

\begin{tabular}{|l|c|c|c|c|}
\cline { 2 - 5 } \multicolumn{1}{c|}{} & \multicolumn{4}{c|}{ K } \\
\cline { 2 - 5 } \multicolumn{1}{c|}{} & Rk4/Splin & Rk4/Trap & Rk5/Splin & Rk5/Trap \\
\hline CASO 1 & 13 & 10 & 12 & 10 \\
\hline CASO 2 & 11 & 12 & 11 & 10 \\
\hline
\end{tabular}

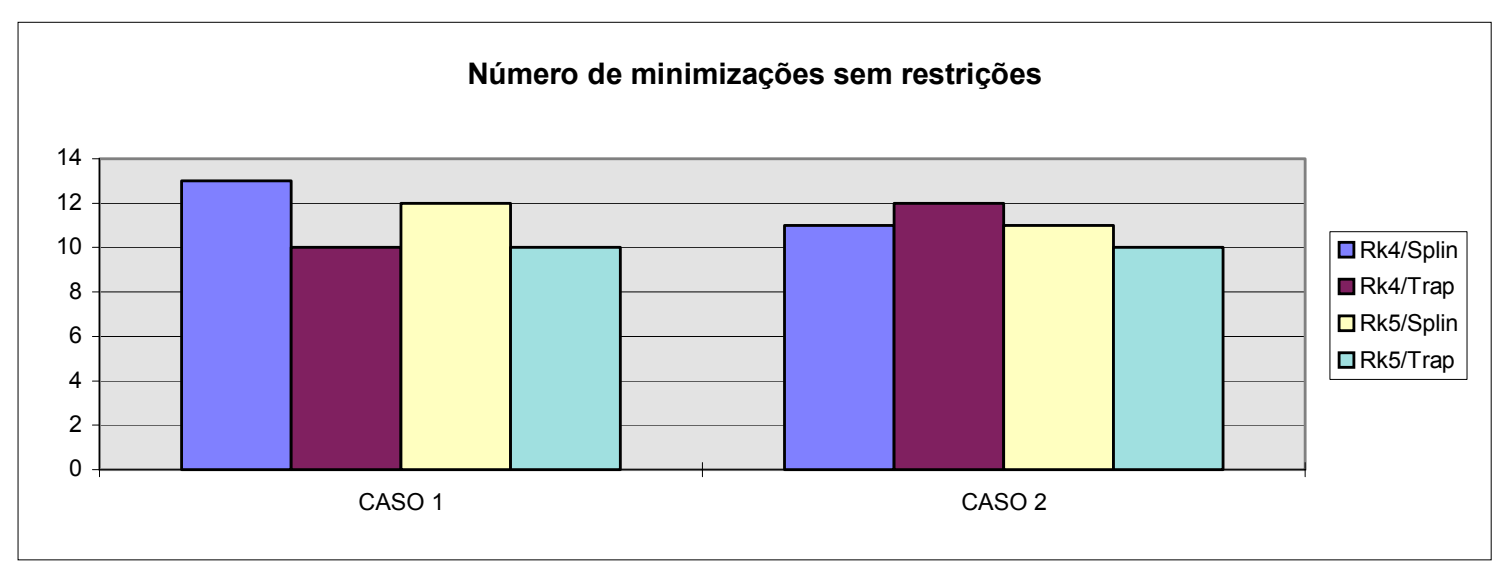

Gráfico 5.17 - Número de minimizações sem restrições para diferentes valores de $\boldsymbol{x}_{0}$ (tabela 5.21).

No gráfico 5.17 mostra que o valor inicial das variáveis de projeto pouco interferiram no número de minimizações sem restrições. Ainda se observa uma leve vantagem da utilização da regra do trapézio à splines cúbicos na integração das violações de retrições. 
Tabela 5.22 - Valores finais das variáveis de projeto para diferentes valores de $\boldsymbol{x}_{0}$.

\begin{tabular}{|c|c|c|c|c|c|c|}
\cline { 3 - 7 } \multicolumn{2}{c}{} & \multicolumn{4}{c|}{ Xf } \\
\cline { 3 - 7 } \multicolumn{2}{c|}{} & $\mathbf{X o}$ & Rk4/Splin & Rk4/Trap & Rk5/Splin & Rk5/Trap \\
\hline \multirow{3}{*}{ CASO 1 } & $\mathbf{X 1}$ & 0,500 & 0,444 & 0,438 & 0,444 & 0,439 \\
\cline { 2 - 7 } & $\mathbf{X 3}$ & 0,500 & 0,522 & 0,526 & 0,523 & 0,525 \\
\cline { 2 - 7 } & $\mathbf{X 1}$ & 0,300 & 0,527 & 0,526 & 0,527 & 0,525 \\
\hline \multirow{3}{*}{ CASO 2 } & $\mathbf{X 2}$ & 0,200 & 0,544 & 0,399 & 0,779 & 0,438 \\
\cline { 2 - 7 } & $\mathbf{X 3}$ & 0,300 & 0,498 & 0,459 & 0,563 & 0,526 \\
\hline
\end{tabular}

Tabela 5.23 - Valores finais da função objetivo para diferentes valores de $\boldsymbol{x}_{0}$.

\begin{tabular}{|c|c|c|c|c|c|}
\cline { 3 - 6 } \multicolumn{1}{c|}{} & $\mathbf{f ( X o )}$ & Rk4/Splin & Rk4/Trap & Rk5/Splin & Rk5/Trap \\
\hline CASO 1 & 0,400 & 0,527 & 0,526 & 0,527 & 0,525 \\
\hline CASO 2 & 0,300 & 0,581 & 0,527 & 0,660 & 0,526 \\
\hline
\end{tabular}

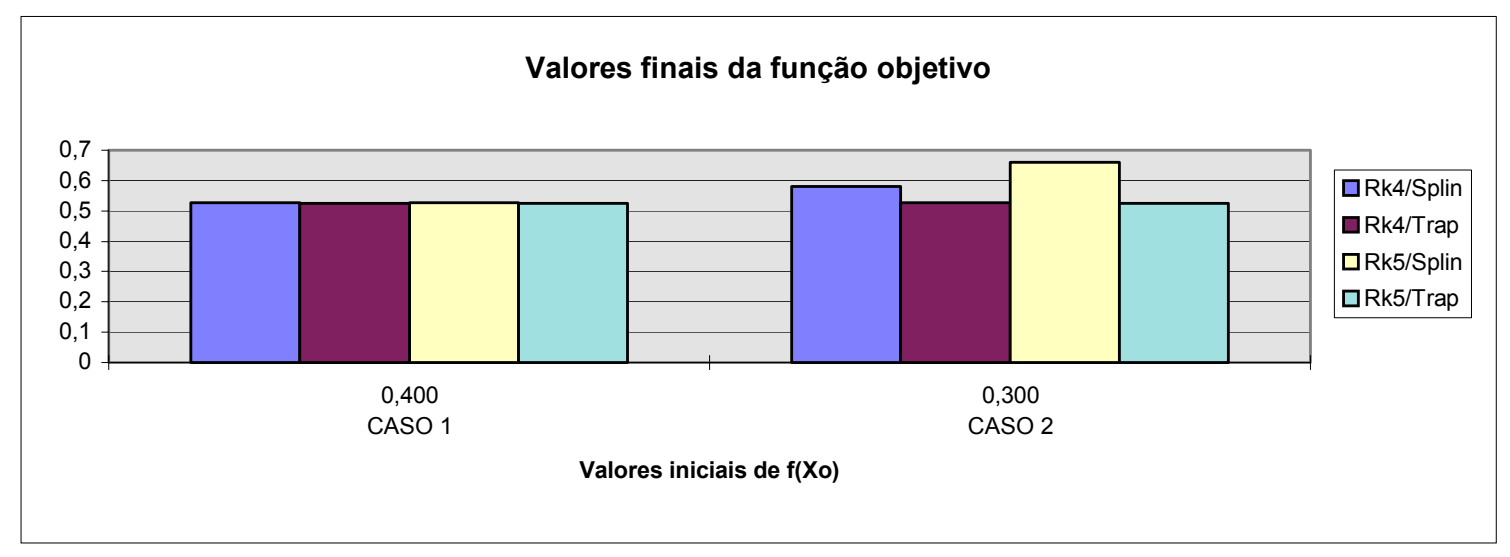

Gráfico 5.18 - Valores finais da função objetivo para diferentes valores de $\boldsymbol{x}_{\mathrm{o}}$ (tabela 5.23).

Observa-se no gráfico acima que no caso 1 o valor da função objetivo foi praticamente o mesmo para todas as combinações de métodos, enquanto que no caso 2 a combinação Runge-Kutta/Trapézio mostra-se mais eficiente.

Tabela 5.24 - Número de minimizações sem restrições para diferentes valores de $\boldsymbol{x}_{0}$.

\begin{tabular}{|l|c|c|c|c|}
\cline { 2 - 5 } \multicolumn{1}{c|}{} & \multicolumn{4}{c|}{$\mathbf{K}$} \\
\cline { 2 - 5 } \multicolumn{1}{c|}{} & Rk4/Splin & Rk4/Trap & Rk5/Splin & Rk5/Trap \\
\hline CASO 1 & 11 & 10 & 11 & 10 \\
\hline CASO 2 & 10 & 10 & 12 & 10 \\
\hline
\end{tabular}


O gráfico abaixo mostra novamente um melhor desempenho das combinações de métodos com a regra do trapézio.

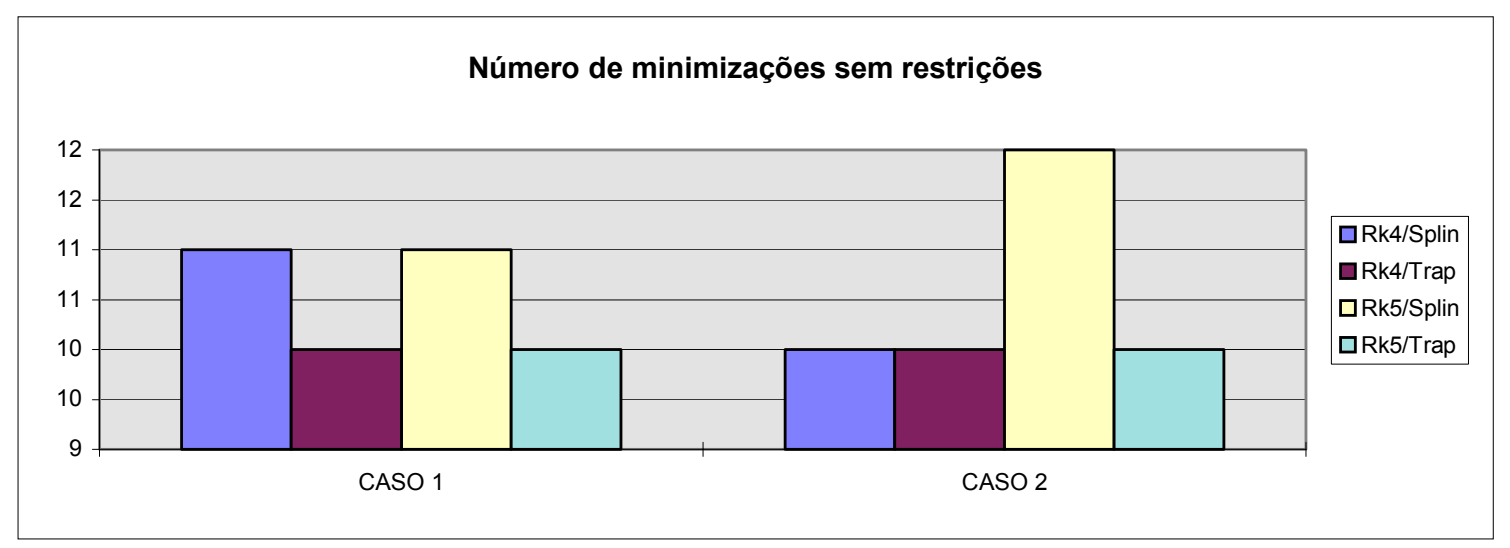

Gráfico 5.19 - Número de minimizações sem restrições para diferentes valores de $\boldsymbol{x}_{\mathrm{0}}$ (tabela 5.24).

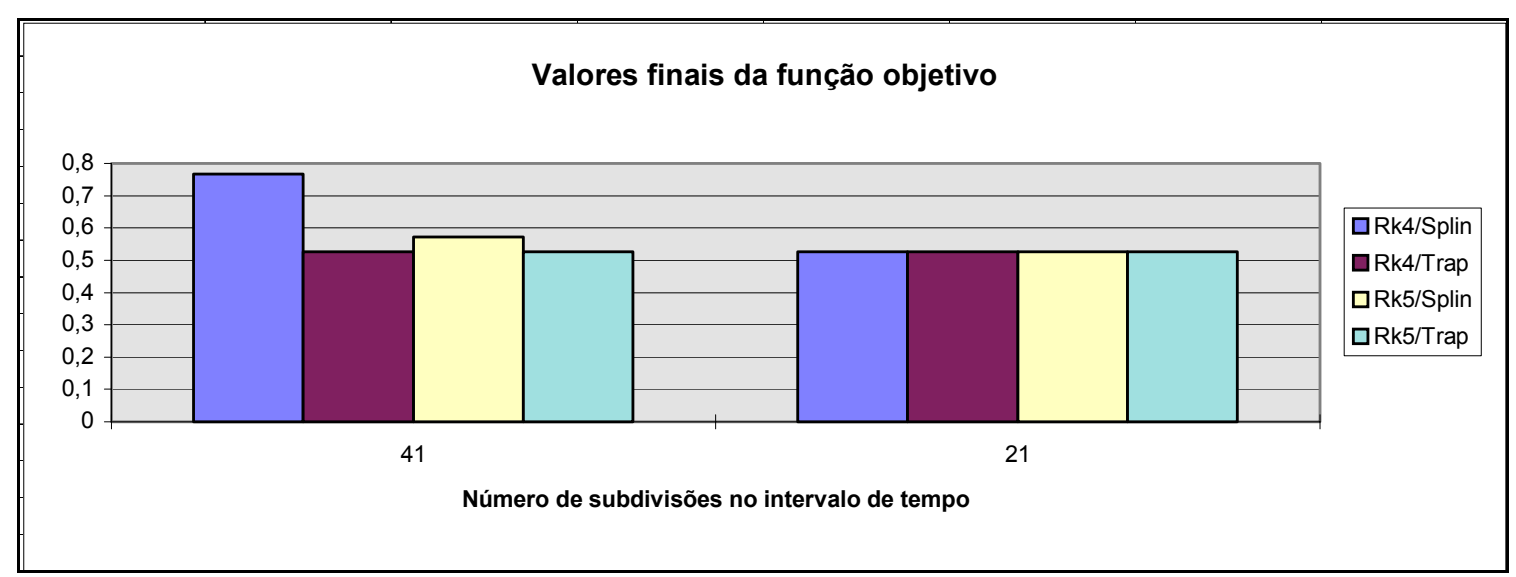

Gráfico 5.20 - Valores finais da função objetivo no caso 1 para diferentes valores de $n$.

O gráfico acima mostra que as combinações com a regra do trapézio mantiveram o valor da função objetivo para os dois numeros de subdivisões no intervalo de tempo, enquanto que as combinações com splines sofreram variações.

No caso de $\omega=2$, o exemplo foi resolvido para dois valores iniciais das variáveis de projeto, os casos 1 e 2 . Um número de 40 subdivisões nas tabelas 5.25 à 5.27 e 20 subdivisões nas tabelas 5.28 à 5.30 foram adotadas na discretização do intervalo de tempo $[0,6] s$. 
Resultados para $\omega=2$

Tabela 5.25 - Valores finais das variáveis de projeto para diferentes valores de $\boldsymbol{x}_{\mathrm{o}}$.

\begin{tabular}{|c|c|c|c|c|c|c|}
\cline { 4 - 7 } \multicolumn{2}{c}{} & \multicolumn{4}{c|}{ Xf } \\
\cline { 3 - 7 } \multicolumn{2}{c|}{} & $\mathbf{X o}$ & Rk4/Splin & Rk4/Trap & Rk5/Splin & Rk5/Trap \\
\hline \multirow{3}{*}{ CASO 1 } & $\mathbf{X 1}$ & 0,500 & 0,599 & 0,597 & 0,618 & 0,597 \\
\cline { 2 - 7 } & $\mathbf{X 2}$ & 0,500 & 0,598 & 0,597 & 0,586 & 0,597 \\
\cline { 2 - 7 } & $\mathbf{X 3}$ & 0,400 & 0,597 & 0,597 & 0,611 & 0,597 \\
\hline \multirow{3}{*}{ CASO 2 } & $\mathbf{X 1}$ & 0,300 & 0,599 & 0,597 & 0,599 & 0,597 \\
\cline { 2 - 7 } & $\mathbf{X 2}$ & 0,200 & 0,598 & 0,597 & 0,597 & 0,597 \\
\cline { 2 - 7 } & $\mathbf{X 3}$ & 0,300 & 0,597 & 0,597 & 0,598 & 0,597 \\
\hline
\end{tabular}

Tabela 5.26 - Valores finais da função objetivo para diferentes valores de $\boldsymbol{x}_{0}$.

\begin{tabular}{|c|c|c|c|c|c|}
\cline { 3 - 6 } \multicolumn{1}{c|}{} & $\mathbf{f ( X o )}$ & Rk4/Splin & Rk4/Trap & Rk5/Splin & Rk5/Trap \\
\hline CASO 1 & 0,400 & 0,597 & 0,597 & 0,611 & 0,597 \\
\hline CASO 2 & 0,300 & 0,597 & 0,597 & 0,598 & 0,597 \\
\hline
\end{tabular}

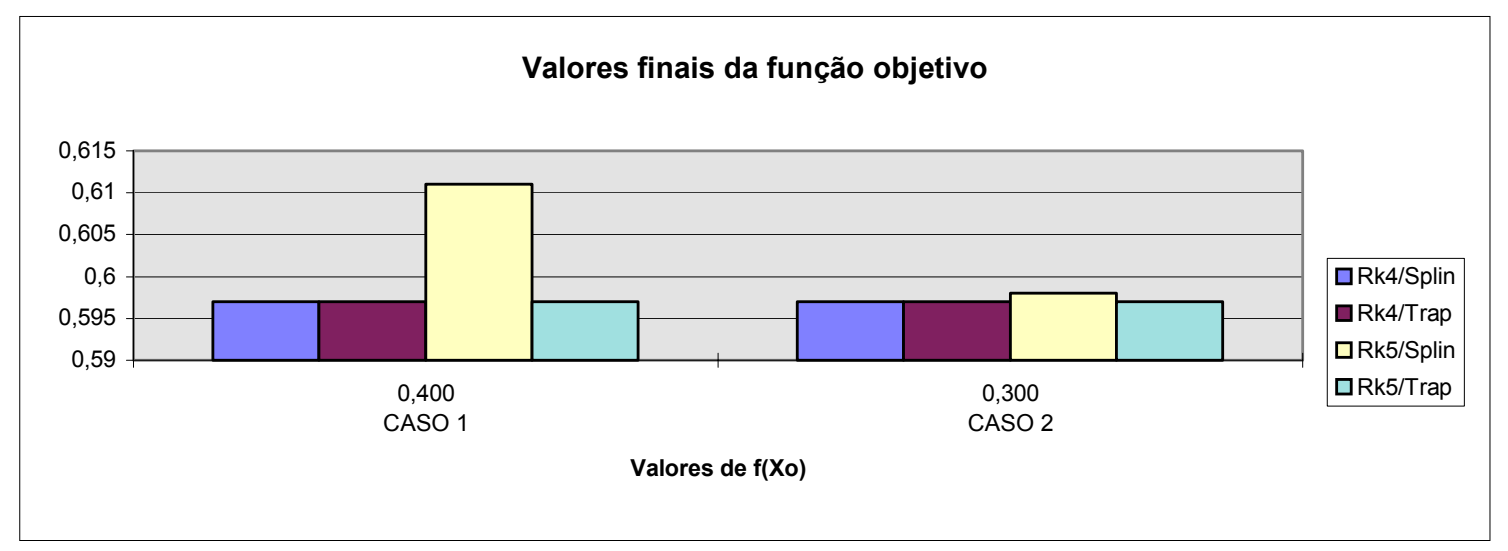

Gráfico 5.21 - Valores finais da função objetivo para diferentes valores de $\boldsymbol{x}_{\mathrm{o}}$ (tabela 5.26).

O gráfico acimo mostra uma leve vatagem das combinções dos métodos com a regra do trapézio que com splines cúbicos.

Tabela 5.27 - Número de minimizações sem restrições para diferentes valores de $\boldsymbol{x}_{0}$.

\begin{tabular}{|l|c|c|c|c|}
\cline { 2 - 5 } \multicolumn{1}{c|}{} & \multicolumn{4}{c|}{ K } \\
\cline { 2 - 5 } \multicolumn{1}{c|}{} & Rk4/Splin & Rk4/Trap & Rk5/Splin & Rk5/Trap \\
\hline CASO 1 & 9 & 10 & 15 & 10 \\
\hline CASO 2 & 9 & 10 & 10 & 10 \\
\hline
\end{tabular}




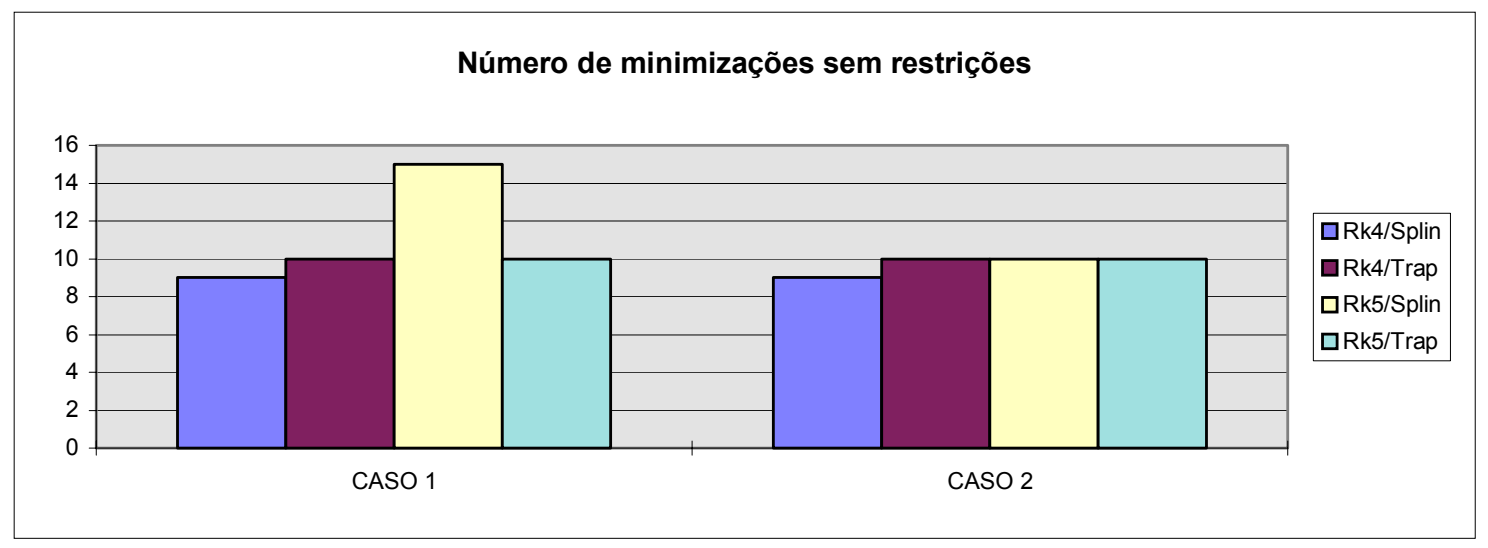

Gráfico 5.22 - Número de minimizações sem restrições para diferentes valores de $\boldsymbol{x}_{0}$ (tabela 5.27).

Já os gráficos 5.22, 5.23 e 5.24 mostram desempenhos equivalentes para as combinações dos diversos métodos, tanto para o caso 1 quanto para o caso 2.

Tabela 5.28 - Valores finais das variáveis de projeto para diferentes valores de $\boldsymbol{x}_{\mathrm{0}}$.

\begin{tabular}{|c|c|c|c|c|c|c|}
\cline { 3 - 7 } \multicolumn{2}{c|}{} & \multicolumn{4}{c|}{$\mathbf{X f}$} \\
\cline { 3 - 7 } \multicolumn{2}{c|}{} & $\mathbf{X o}$ & Rk4/Splin & Rk4/Trap & Rk5/Splin & Rk5/Trap \\
\hline \multirow{3}{*}{ CASO 1 } & $\mathbf{X 1}$ & 0,500 & 0,761 & 0,658 & 0,791 & 0,589 \\
\cline { 2 - 7 } & $\mathbf{X 2}$ & 0,500 & 0,820 & 0,616 & 0,561 & 0,593 \\
\cline { 2 - 7 } & $\mathbf{X 3}$ & 0,400 & 0,819 & 0,616 & 0,701 & 0,593 \\
\hline \multirow{3}{*}{ CASO 2 } & $\mathbf{X 1}$ & 0,300 & 0,698 & 0,589 & 0,857 & 0,589 \\
\cline { 2 - 7 } & $\mathbf{X 2}$ & 0,200 & 0,536 & 0,593 & 0,430 & 0,593 \\
\cline { 2 - 7 } & $\mathbf{X 3}$ & 0,300 & 0,676 & 0,593 & 0,801 & 0,593 \\
\hline
\end{tabular}

Tabela 5.29 - Valores finais da função objetivo para diferentes valores de $\boldsymbol{x}_{0}$.

\begin{tabular}{|c|c|c|c|c|c|}
\cline { 3 - 6 } \multicolumn{1}{c|}{} & $\mathbf{f ( X o )}$ & Rk4/Splin & Rk4/Trap & Rk5/Splin & Rk5/Trap \\
\cline { 2 - 6 } \multicolumn{1}{c|}{} & 0,400 & 0,819 & 0,616 & 0,701 & 0,593 \\
\hline CASO 1 & 0,300 & 0,676 & 0,593 & 0,801 & 0,593 \\
\hline
\end{tabular}




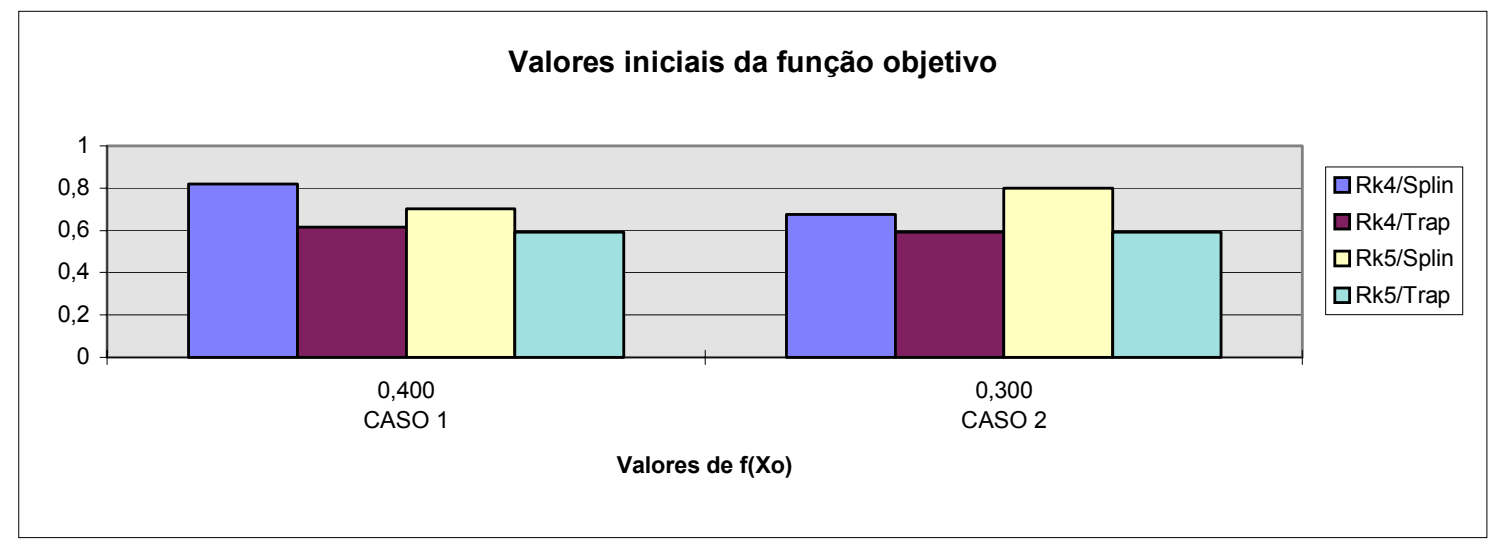

Gráfico 5.23 - Valores finais da função objetivo para diferentes valores de $\boldsymbol{x}_{\mathbf{0}}$ (tabela 5.29).

Tabela 5.30 - Número de minimizações sem restrições para diferentes valores de $\boldsymbol{x}_{0}$.

\begin{tabular}{|l|c|c|c|c|}
\cline { 2 - 5 } \multicolumn{1}{c|}{} & \multicolumn{4}{c|}{ K } \\
\cline { 2 - 5 } \multicolumn{1}{c|}{} & Rk4/Splin & Rk4/Trap & Rk5/Splin & Rk5/Trap \\
\hline CASO 1 & 9 & 10 & 14 & 9 \\
\hline CASO 2 & 9 & 11 & 12 & 9 \\
\hline
\end{tabular}

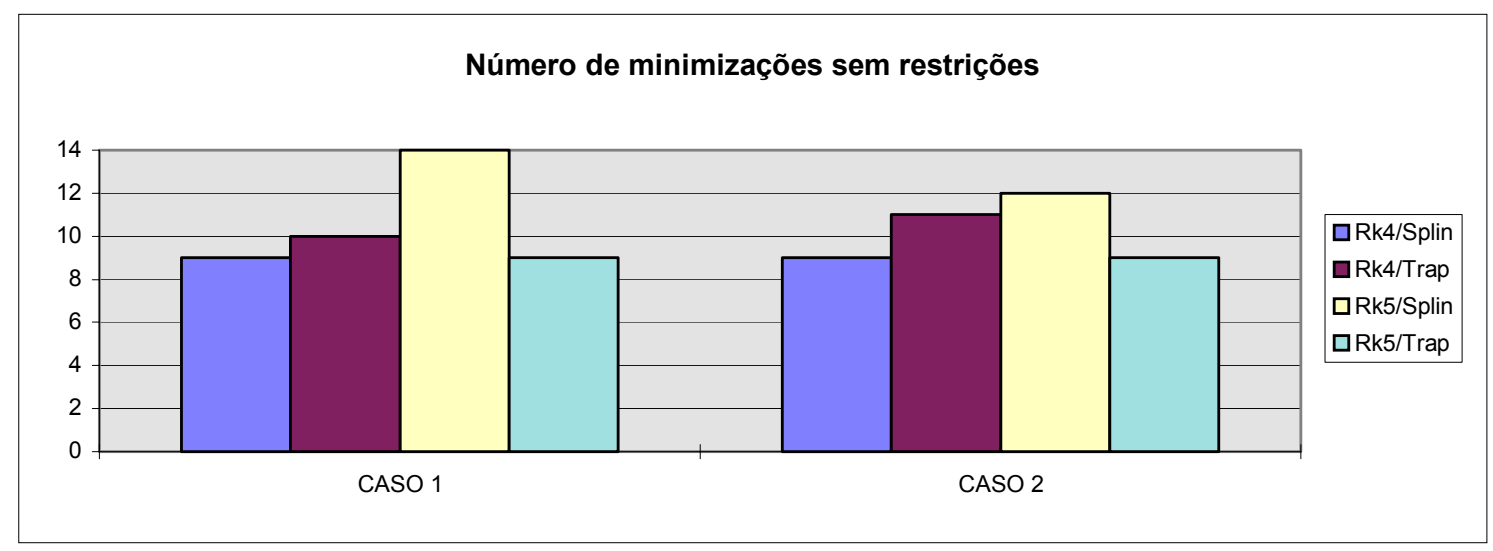

Gráfico 5.24 - Número de minimizações sem restrições para diferentes valores de $\boldsymbol{x}_{0}$ (tabela 5.30).

O gráfico abaixo mostra uma pequena vantagem das combinações com o método do trapézio sobre as combinações com splines cúbicos. 


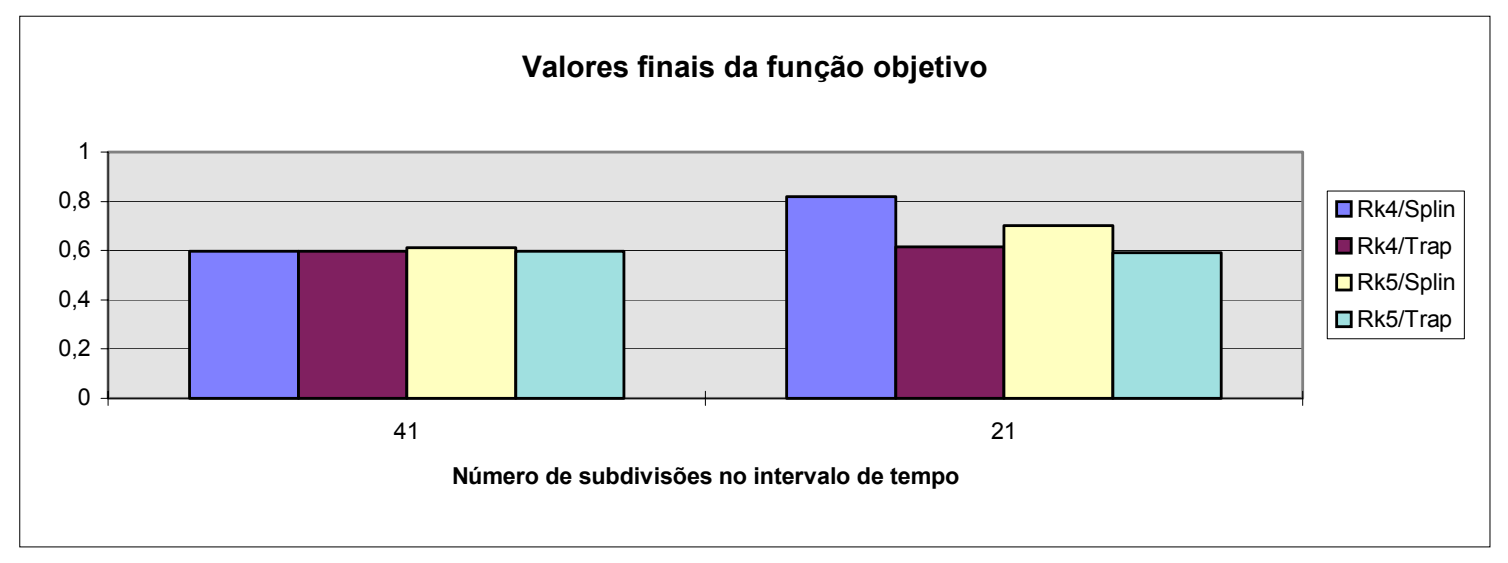

Gráfico 5.25 - Valores finais da função objetivo no caso 1 para diferentes valores de $n$.

No caso de $\omega=3$, o exemplo foi resolvido para dois valores iniciais das variáveis de projeto, os casos 1 e 2. Um número de 40 subdivisões nas tabelas 5.31 à 5.33 e 20 subdivisões nas tabelas 5.34 à 5.36 foram adotadas na discretização do intervalo de tempo $[0,6] s$.

Resultados para $\omega=3$

Tabela 5.31 - Valores finais das variáveis de projeto para diferentes valores de $\boldsymbol{x}_{\mathrm{0}}$.

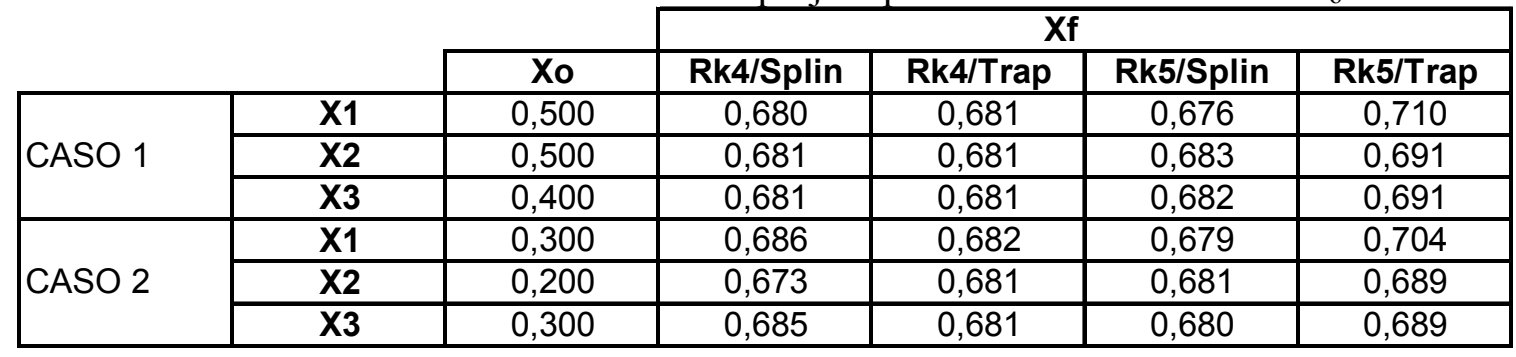

Tabela 5.32 - Valores finais da função objetivo para diferentes valores de $\boldsymbol{x}_{0}$.

\begin{tabular}{|c|c|c|c|c|c|}
\cline { 3 - 6 } \multicolumn{1}{c|}{} & $\mathbf{f ( X o )}$ & Rk4/Splin & Rk4/Trap & Rk5/Splin & Rk5/Trap \\
\hline CASO 1 & 0,400 & 0,681 & 0,681 & 0,682 & 0,691 \\
\hline CASO 2 & 0,300 & 0,685 & 0,681 & 0,680 & 0,689 \\
\hline
\end{tabular}




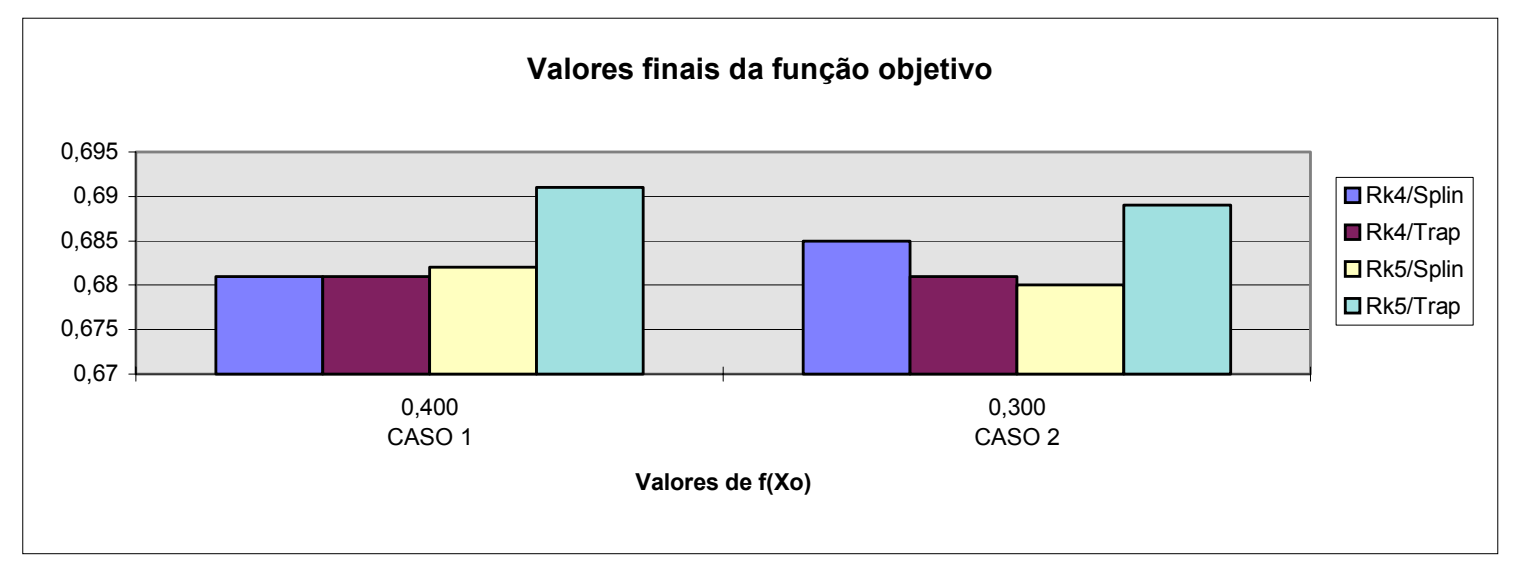

Gráfico 5.26 - Valores finais da função objetivo para diferentes valores de $\boldsymbol{x}_{\mathrm{o}}$ (tabela 5.32).

O gráfico acima mostra uma pequena vantagem das combinações com trapézio sobre as com splines cúbicos e que o valor inicial das variáveis de projeto pouco interfere no desempenho dos métodos.

Tabela 5.33 - Número de minimizações sem restrições para diferentes valores de $\boldsymbol{x}_{0}$.

\begin{tabular}{|l|c|c|c|c|}
\cline { 2 - 5 } \multicolumn{1}{c|}{} & \multicolumn{4}{c|}{$\mathbf{K}$} \\
\cline { 2 - 5 } \multicolumn{1}{c|}{} & Rk4/Splin & Rk4/Trap & Rk5/Splin & Rk5/Trap \\
\hline CASO 1 & 11 & 10 & 12 & 10 \\
\hline CASO 2 & 8 & 10 & 7 & 10 \\
\hline
\end{tabular}

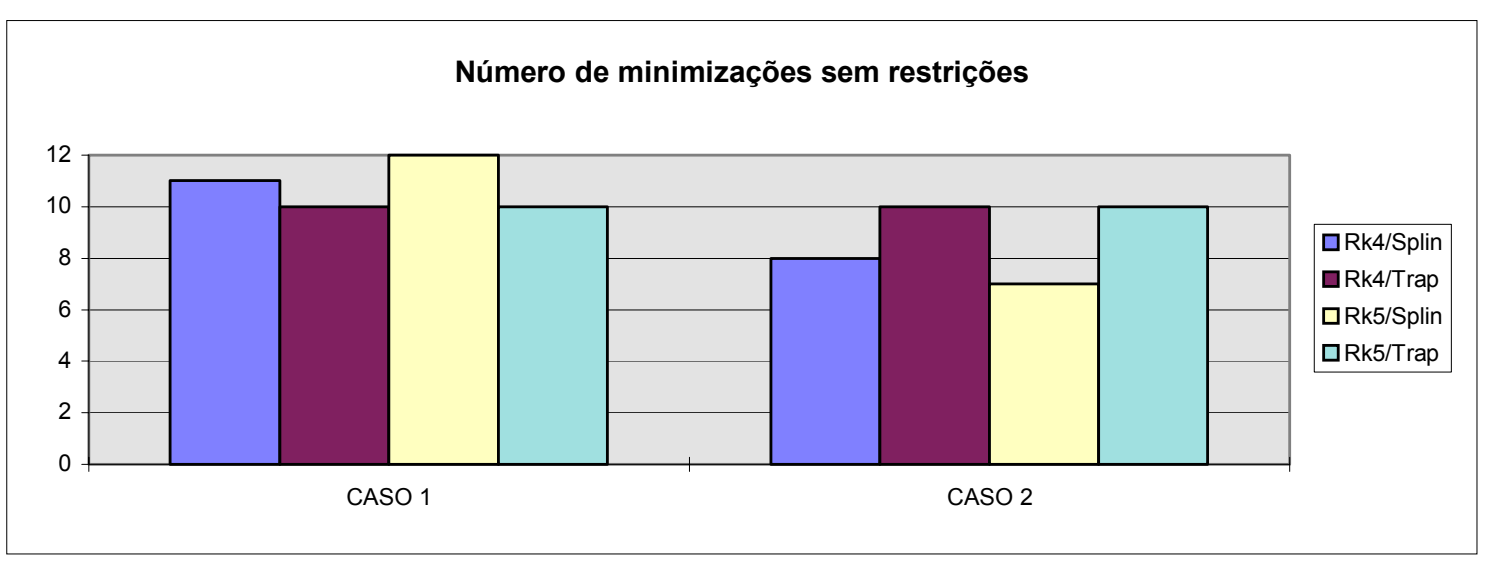

Gráfico 5.27 - Número de minimizações sem restrições para diferentes valores de $\boldsymbol{x}_{\mathrm{o}}$ (tabela 5.33).

O gráfico acima mostra um equilíbrio entre as combinações dos diversos métodos, mas com uma leve sensibilidade ao valor inicial das variáveis de projeto. 
Já no gráfico 5.28 verifica-se que as combinações com a regra do trapézio apresenta melhor desempenho que com splines cúbicos.

Tabela 5.34 - Valores finais das variáveis de projeto para diferentes valores de $\boldsymbol{x}_{0}$.

\begin{tabular}{|c|c|c|c|c|c|c|}
\hline & & \multicolumn{4}{|c|}{$\overline{X f}$} \\
\hline & & Xo & Rk4/Splin & Rk4/Trap & Rk5/Splin & Rk5/Trap \\
\hline \multirow{3}{*}{ CASO 1} & $\mathrm{X1}$ & 0,500 & 0,796 & 0,682 & 0,681 & 0,680 \\
\hline & $\mathrm{X} 2$ & 0,500 & 0,712 & 0,681 & 0,679 & 0,680 \\
\hline & $\mathrm{X3}$ & 0,400 & 0,721 & 0,681 & 0,683 & 0,680 \\
\hline \multirow{3}{*}{ CASO 2} & $\mathrm{X1}$ & 0,300 & 0,732 & 0,680 & 0,735 & 0,680 \\
\hline & $\mathrm{X} 2$ & 0,200 & 0,687 & 0,680 & 0,694 & 0,680 \\
\hline & $\mathrm{X3}$ & 0,300 & 0,702 & 0,680 & 0,701 & 0,680 \\
\hline
\end{tabular}

Tabela 5.35 - Valores finais da função objetivo para diferentes valores de $\boldsymbol{x}_{0}$.

\begin{tabular}{|c|c|c|c|c|c|}
\cline { 3 - 6 } \multicolumn{1}{c|}{} & $\mathbf{f ( X o )}$ & Rk4/Splin & Rk4/Trap & Rk5/Splin & Rk5/Trap \\
\hline CASO 1 & 0,400 & 0,721 & 0,681 & 0,683 & 0,680 \\
\hline CASO 2 & 0,300 & 0,702 & 0,680 & 0,701 & 0,680 \\
\hline
\end{tabular}

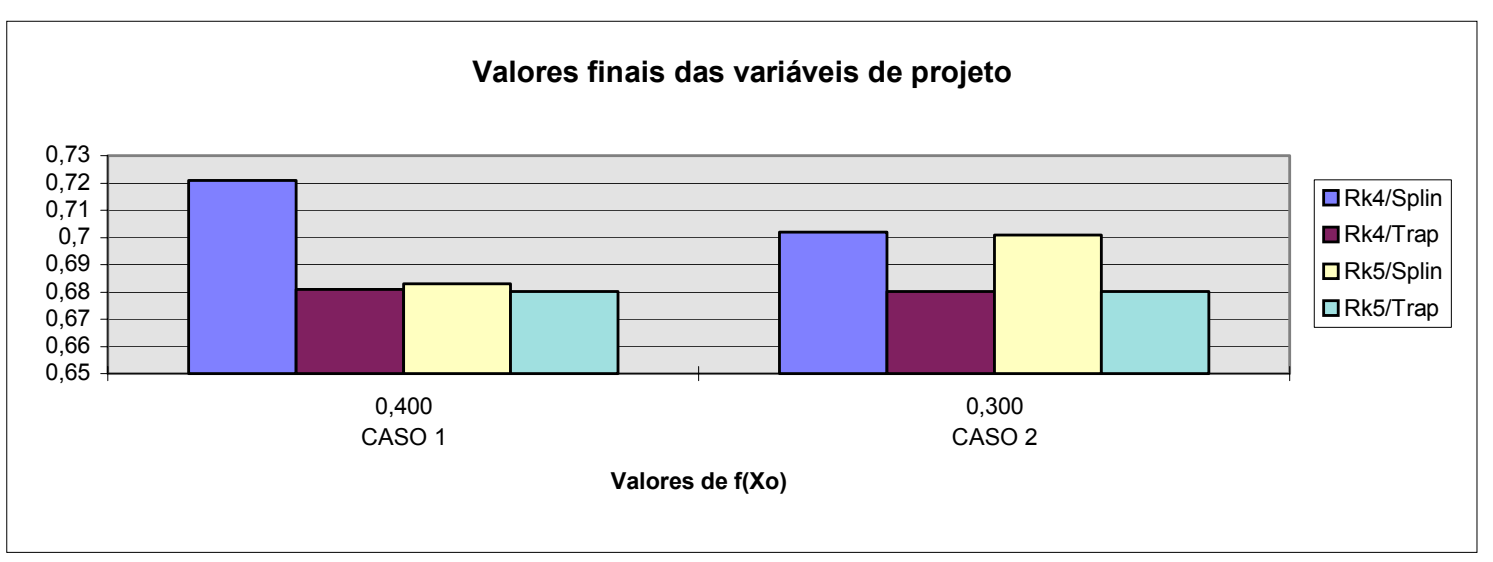

Gráfico 5.28 - Valores finais da função objetivo para diferentes valores de $\boldsymbol{x}_{\mathrm{o}}$ (tabela 5.35).

Tabela 5.36 - Número de minimizações sem restrições para diferentes valores de $\boldsymbol{x}_{0}$.

\begin{tabular}{|l|c|c|c|c|}
\cline { 2 - 5 } \multicolumn{1}{c|}{} & \multicolumn{4}{c|}{ K } \\
\cline { 2 - 5 } \multicolumn{1}{c|}{} & Rk4/Splin & Rk4/Trap & Rk5/Splin & Rk5/Trap \\
\hline CASO 1 & 9 & 10 & 6 & 10 \\
\hline CASO 2 & 127 & 10 & 10 & 10 \\
\hline
\end{tabular}




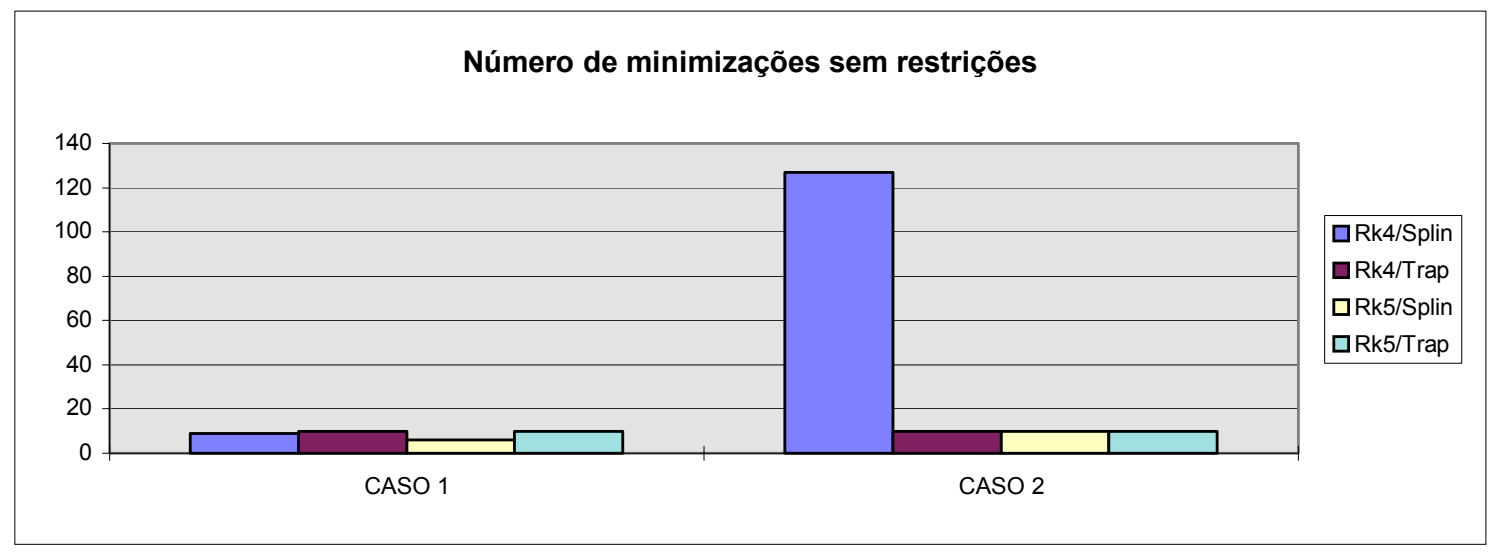

Gráfico 5.29 - Número de minimizações sem restrições para diferentes valores de $\boldsymbol{x}_{\mathrm{o}}$ (tabela 5.36).

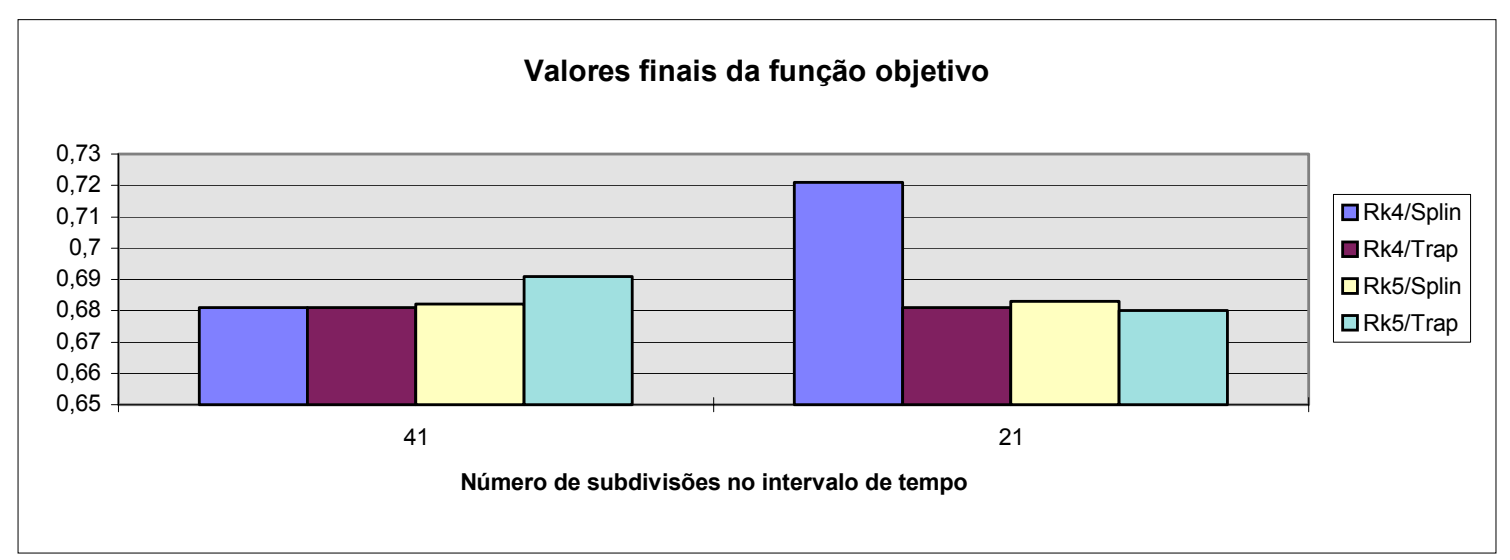

Gráfico 5.30 - Valores finais da função objetivo no caso 1 para diferentes valores de $n$.

No gráfico 5.29 observa-se uma instabilidade na combinação Runge-Kutta de quarta ordem com splines cúbicos para o caso 2. Enquanto que o gráfico 5.30 mostra um equilíbrio entre as diversas combinações de métodos, mostrando ainda uma pequena sensibilidade à variação de $n$.

No caso de $\omega=4$, o exemplo foi resolvido para dois valores iniciais das variáveis de projeto, os casos 1 e 2 . Um número de 40 subdivisões nas tabelas 5.37 à 5.39 e 20 subdivisões nas tabelas 5.40 à 5.42 foram adotadas na discretização do intervalo de tempo $[0,6] s$.

Resultados para $\omega=4$ 
Tabela 5.37 - Valores finais das variáveis de projeto para diferentes valores de $\boldsymbol{x}_{0}$.

\begin{tabular}{|c|c|c|c|c|c|c|}
\cline { 3 - 7 } \multicolumn{2}{c}{} & \multicolumn{4}{c|}{ Xf } \\
\cline { 3 - 7 } \multicolumn{2}{c|}{} & $\mathbf{X o}$ & Rk4/Splin & Rk4/Trap & Rk5/Splin & Rk5/Trap \\
\hline \multirow{3}{*}{ CASO 1 } & $\mathbf{X 1}$ & 0,500 & 0,841 & 0,853 & 0,850 & 0,754 \\
\cline { 2 - 7 } & $\mathbf{X 2}$ & 0,500 & 0,781 & 0,783 & 0,781 & 0,754 \\
\cline { 2 - 7 } & $\mathbf{X 3}$ & 0,400 & 0,781 & 0,785 & 0,784 & 0,754 \\
\hline \multirow{3}{*}{ CASO 2 } & $\mathbf{X 1}$ & 0,300 & 0,779 & 0,764 & 0,756 & 0,754 \\
\cline { 2 - 7 } & $\mathbf{X 2}$ & 0,200 & 0,761 & 0,757 & 0,754 & 0,753 \\
\cline { 2 - 7 } & $\mathbf{X 3}$ & 0,300 & 0,762 & 0,757 & 0,754 & 0,753 \\
\hline
\end{tabular}

Tabela 5.38 - Valores finais da função objetivo para diferentes valores de $\boldsymbol{x}_{0}$.

\begin{tabular}{|c|c|c|c|c|c|}
\cline { 3 - 6 } \multicolumn{1}{c|}{} & $\mathbf{f ( X o )}$ & Rk4/Splin & Rk4/Trap & Rk5/Splin & Rk5/Trap \\
\hline CASO 1 & 0,400 & 0,781 & 0,785 & 0,784 & 0,754 \\
\hline CASO 2 & 0,300 & 0,762 & 0,757 & 0,754 & 0,753 \\
\hline
\end{tabular}

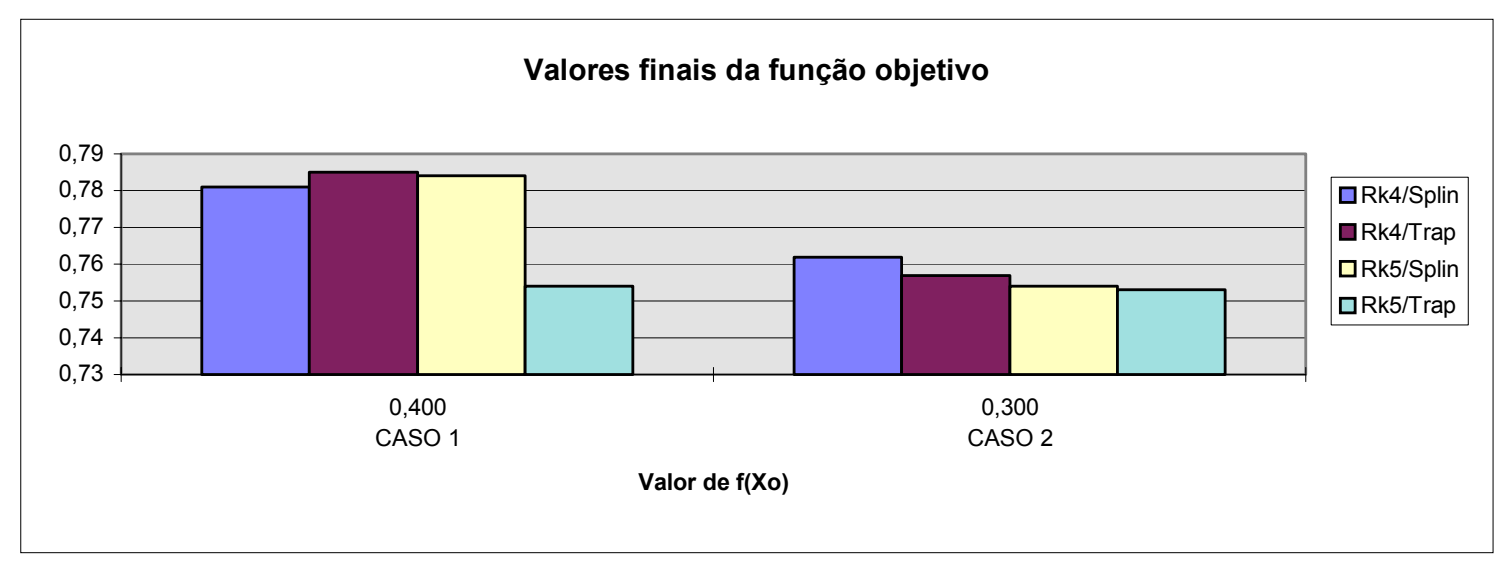

Gráfico 5.31 - Valores finais da função objetivo para diferentes valores de $\boldsymbol{x}_{\mathrm{o}}$ (tabela 5.38).

O gráfico acima mostra uma pequena sensibilidade nos valores finais da função objetivo em relação à variação do valor inicial de $\boldsymbol{x}$.

Tabela 5.39 - Número de minimizações sem restrições para diferentes valores de $\boldsymbol{x}_{0}$.

\begin{tabular}{|l|c|c|c|c|}
\cline { 2 - 5 } \multicolumn{1}{c|}{} & \multicolumn{4}{c|}{$\mathbf{K}$} \\
\cline { 2 - 5 } \multicolumn{1}{c|}{} & Rk4/Splin & Rk4/Trap & Rk5/Splin & Rk5/Trap \\
\hline CASO 1 & 10 & 10 & 8 & 10 \\
\hline CASO 2 & 12 & 10 & 7 & 10 \\
\hline
\end{tabular}




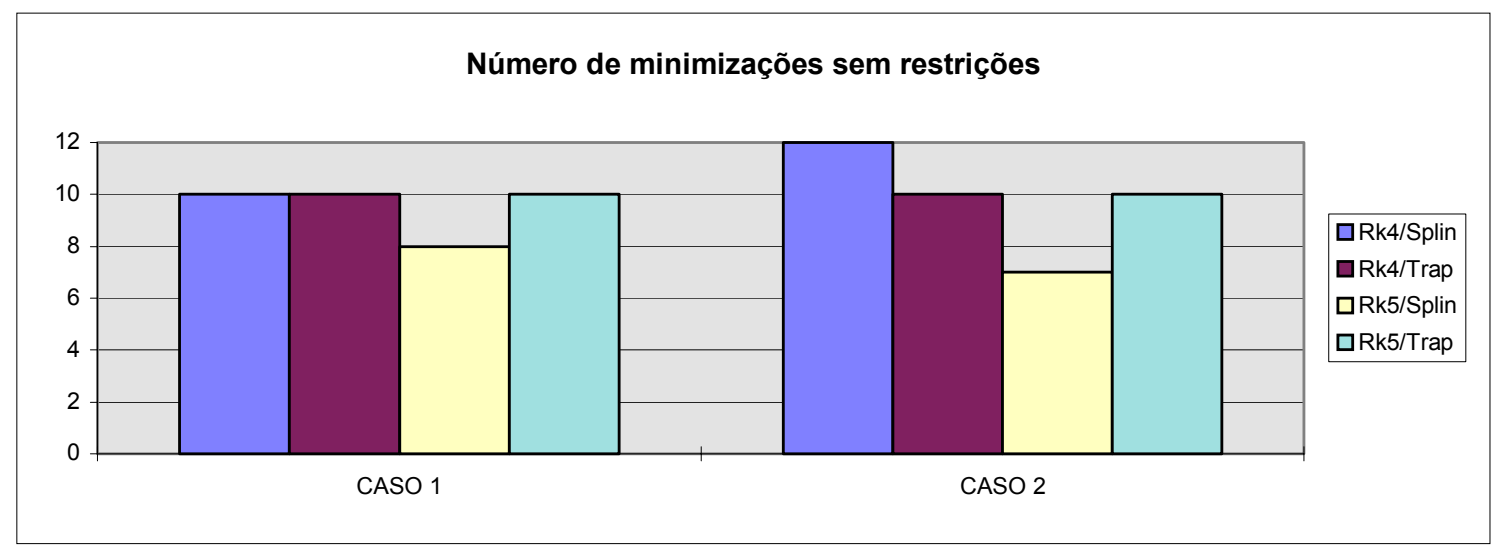

Gráfico 5.32 - Número de minimizações sem restrições para diferentes valores de $\boldsymbol{x}_{\mathrm{o}}$ (tabela 5.39).

Verifica-se um equilíbrio entre as diversas combinações dos métodos no gráfico acima, tanto para o caso 1 quanto para o 2 .

Tabela 5.40 - Valores finais das variáveis de projeto para diferentes valores de $\boldsymbol{x}_{0}$.

\begin{tabular}{|c|c|c|c|c|c|c|}
\cline { 3 - 7 } \multicolumn{2}{c|}{} & \multicolumn{4}{c|}{ Xf } \\
\cline { 3 - 7 } \multicolumn{2}{c|}{} & $\mathbf{X o}$ & Rk4/Splin & Rk4/Trap & Rk5/Splin & Rk5/Trap \\
\hline \multirow{3}{*}{ CASO 1 } & $\mathbf{X 1}$ & 0,500 & 0,915 & 0,854 & 0,868 & 0,754 \\
\cline { 2 - 7 } & $\mathbf{X 2}$ & 0,500 & 0,799 & 0,770 & 0,784 & 0,753 \\
\cline { 2 - 7 } & $\mathbf{X 3}$ & 0,400 & 0,800 & 0,789 & 0,789 & 0,754 \\
\hline \multirow{3}{*}{ CASO 2 } & $\mathbf{X 1}$ & 0,300 & 0,866 & 0,753 & 0,756 & 0,755 \\
\cline { 2 - 7 } & $\mathbf{X 2}$ & 0,200 & 0,774 & 0,753 & 0,744 & 0,754 \\
\cline { 2 - 7 } & $\mathbf{X 3}$ & 0,300 & 0,791 & 0,753 & 0,756 & 0,754 \\
\hline
\end{tabular}

Tabela 5.41 - Valores finais da função objetivo para diferentes valores de $\boldsymbol{x}_{0}$.

\begin{tabular}{|c|c|c|c|c|c|}
\cline { 3 - 6 } \multicolumn{1}{c|}{} & $\mathbf{f ( X o )}$ & Rk4/Splin & Rk4/Trap & Rk5/Splin & Rk5/Trap \\
\hline CASO 1 & 0,400 & 0,800 & 0,789 & 0,789 & 0,754 \\
\hline CASO 2 & 0,300 & 0,791 & 0,753 & 0,756 & 0,754 \\
\hline
\end{tabular}

Já no gráfico 5.33 observa-se uma pequena vantagem das combinações com trapézio que com splines cúbicos. A mesma situação é também verificada no gráfico 5.34. 


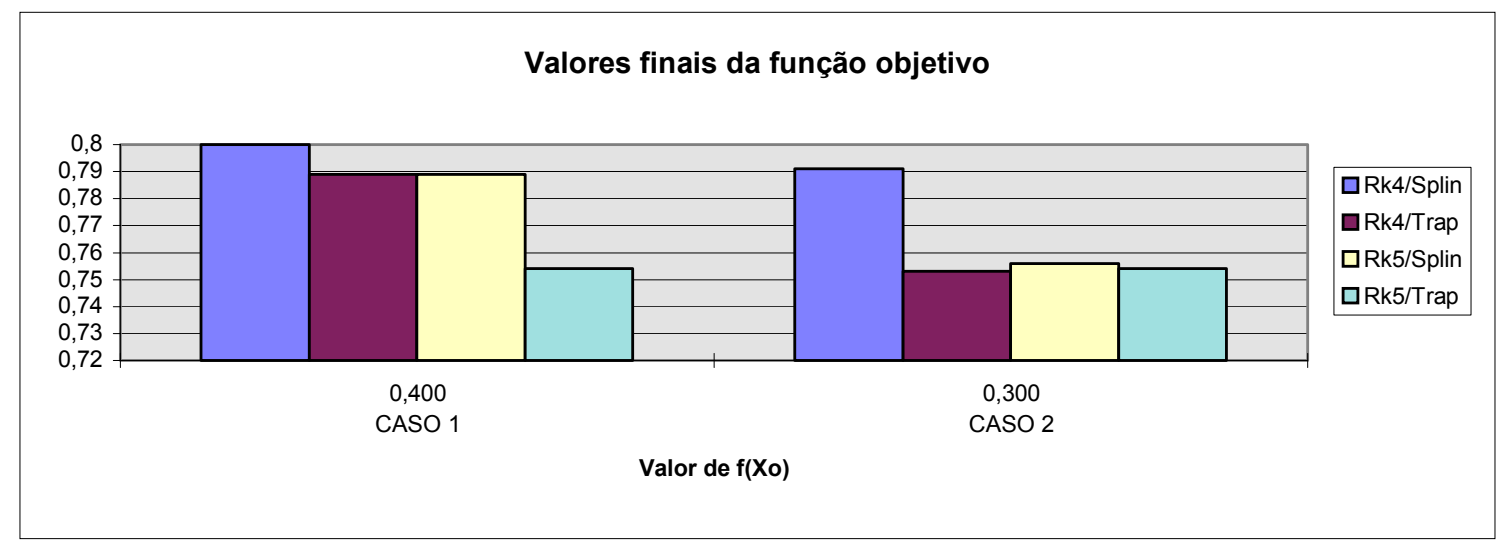

Gráfico 5.33 - Valores finais da função objetivo para diferentes valores de $\boldsymbol{x}_{\mathrm{o}}$ (tabela 5.41).

Tabela 5.42 - Número de minimizações sem restrições para diferentes valores de $\boldsymbol{x}_{0}$.

\begin{tabular}{|l|c|c|c|c|}
\cline { 2 - 5 } \multicolumn{1}{c|}{} & \multicolumn{4}{c|}{ K } \\
\cline { 2 - 5 } \multicolumn{1}{c|}{} & Rk4/Splin & Rk4/Trap & Rk5/Splin & Rk5/Trap \\
\hline CASO 1 & 10 & 10 & 11 & 10 \\
\hline CASO 2 & 12 & 10 & 10 & 10 \\
\hline
\end{tabular}

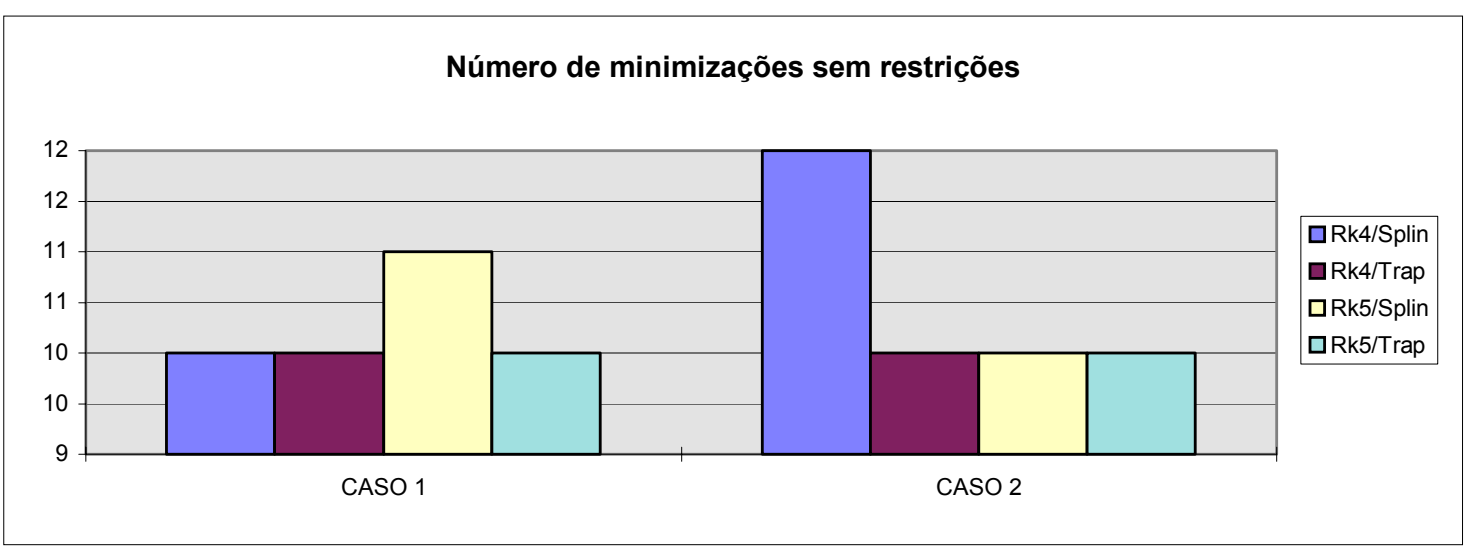

Gráfico 5.34 - Número de minimizações sem restrições para diferentes valores de $\boldsymbol{x}_{\mathrm{o}}$ (tabela 5.42).

O gráfico 5.35 não mostra praticamente nenhuma sensibilidade das combinações dos métodos em relação à variação do número de subdivisões no intervalo de tempo, porém as combinações com trapézio obtiveram um melhor desempenho. 


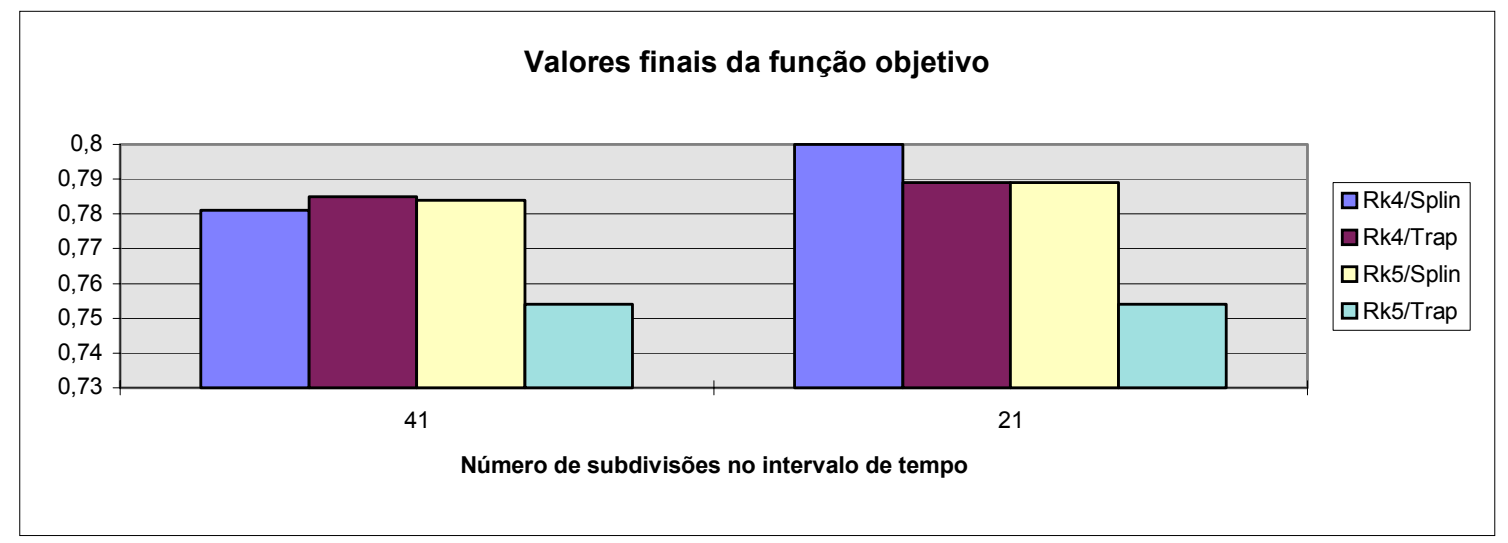

Gráfico 5.35 - Valores finais da função objetivo no caso 1 para diferentes valores de $n$. 


\subsection{EXEMPLO 5 (Haug e Arora [7])}

\section{Otimização de um isolador de vibração linear com dois graus de liberdade}

Um bom exemplo da aplicação do Método dos Multiplicadores em problemas com resposta dinâmica é o isolador de vibração.A fundação de motor da figura 5.5 apresentava problemas de vibração, engenheiros tentando resolver este problema acrescentaram ao problema mais um grau de liberdade, o deslocamento vertical da massa $m_{2}$. Na prática esta massa tem duas principais funções: a) dissipar a energia em forma de vibração emitida pelo motor elétrico da figura abaixo, reduzindo assim o deslocamento imposto à fundação pelo motor; b) fazer com que as frequências de vibração natural do sistema assumam valores diferentes do valor da frequência excitadora.

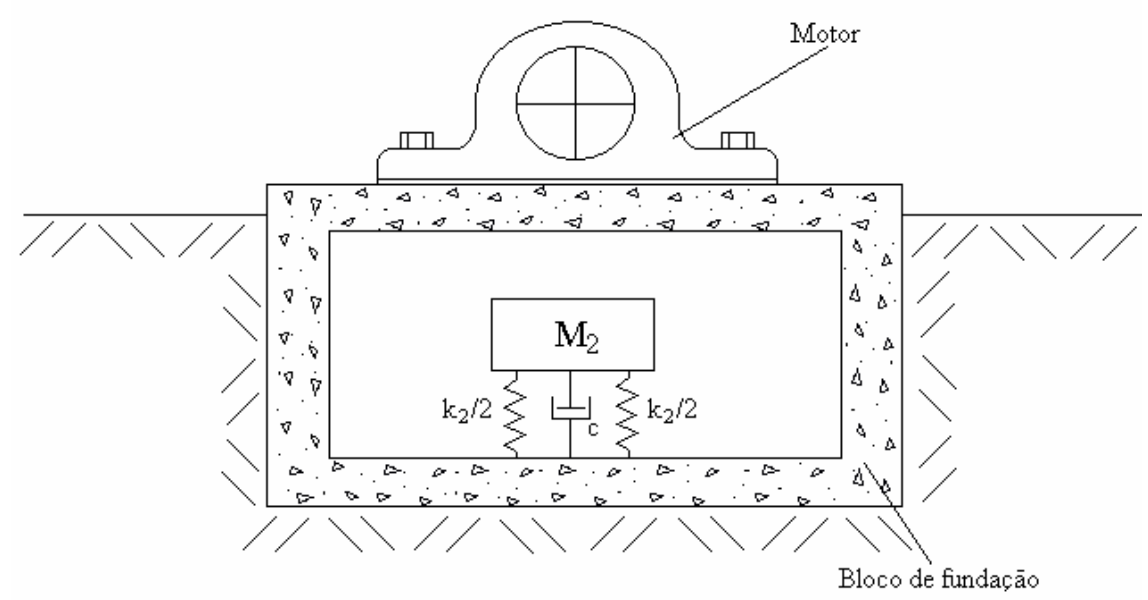

Figura 5.5. Isolador de vibração.

Um modelo matemático deste isolador de vibração é mostrado na figura 5.6, onde os deslocamentos do sistema são $b_{1}$ e $b_{2} . m_{1}$ é igual à massa do bloco de fundação somada à massa do motor e $k_{1}$ é a rigidez do solo. Define-se deslocamentos admensionais $z_{1}=b_{1} / b_{\text {st }} \mathrm{e}$ $z_{2}=b_{2} / b_{\text {st }}$, onde $b_{s t}=F / k_{1}$ é o deslocamento solução para o problema estático.

Os deslocamentos $z_{1}$ e $z_{2}$ devem satisfazer o sistema de equações diferenciais ordinárias lineares

$$
\begin{aligned}
& \ddot{z}_{1}+\Omega_{n}^{2} z_{1}+f^{2} \Omega_{n}^{2} \mu\left(z_{1}-z_{2}\right)+2 \Omega_{n} \mu \xi\left(\dot{z}_{1}-\dot{z}_{2}\right)=\Omega_{n}^{2} \sin \left(\Omega_{n} \zeta t\right) \\
& \ddot{z_{2}}+f^{2} \Omega_{n}^{2}\left(z_{2}-z_{1}\right)+2 \Omega_{n} \xi\left(\dot{z}_{2}-\dot{z}_{1}\right)=0
\end{aligned}
$$


para todo $t \in[0,0.667] s$. As condições iniciais são $\boldsymbol{z}(0)=\mathbf{0}$ e $\boldsymbol{z}^{\prime}(0)=\mathbf{0}$.

As amplitudes de vibração no regime estacionário são

$$
\begin{aligned}
& z_{1 s}=\left[\frac{(2 \zeta \xi)^{2}+\left(\zeta^{2}-f^{2}\right)^{2}}{z_{b}}\right]^{1 / 2} \mathrm{e} \\
& z_{2 s}=\left[\frac{(2 \zeta \xi)^{2}+f^{4}}{z_{b}}\right]^{1 / 2}, \text { onde } \\
& z_{b}=(2 \zeta \xi)^{2}\left(\zeta^{2}-1+\mu \zeta^{2}\right)^{2}+\left[\mu f^{2} \zeta^{2}-\left(\zeta^{2}-1\right)\left(\zeta^{2}-f^{2}\right)\right]^{2}
\end{aligned}
$$

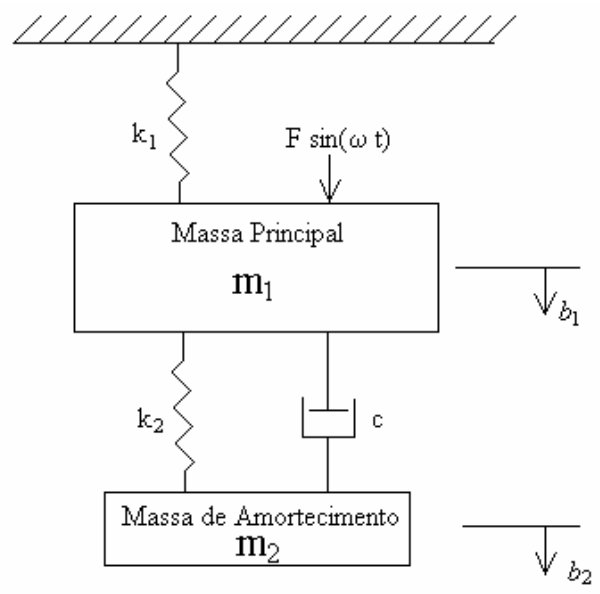

Figura 5.6 Modelo matemático de um isolador de vibração

O objetivo consiste em determinar constantes elásticas através da variável $f=x_{1}=\sqrt{\left(k_{2} m_{1}\right) /\left(k_{1} m_{2}\right)}$ e do amortecimento viscoso $\xi=x_{2}=c / \sqrt{\left(2 m_{2}\left(k_{1} m_{1}\right)\right.}$ de modo a minimizar $x_{3}$, variável artificial de projeto, que representa o máximo deslocamento da massa principal. O sistema é excitado com frequência $\omega=\Omega_{n} \zeta$, sendo que $\Omega_{n}=\sqrt{k_{1} / m_{1}}$ é uma freqüência de oscilação natural do sistema. Se $\zeta=1$ o sistema entrará em ressonância, posto isso neste trabalho adotou-se $\zeta=1.2$. A razão entre as massas é $\mu=m_{2} / m_{1}$. Restrições são impostas aos deslocamentos nos regimes transiente e estacionário.

O problema de otimização pode ser apresentado na forma: 
Determine $x_{1}, x_{2}$ e $x_{3}$ que minimize a função objetivo

$$
f(\boldsymbol{x})=x_{3}
$$

sujeito às restrições dinâmicas

$$
\left|z_{1}(t)\right|-x_{3} \leq 0, \text { para todo } t \in[0,0.667] s
$$

$\mathrm{e}$

$$
\left|z_{2}(t)-z_{1}(t)\right|-2 x_{3} \leq 0, \text { para todo } t \in[0,0.667] s
$$

Os deslocamentos no regime estacionário são restritos à

$$
\begin{aligned}
& z_{1 s}(f, \xi, \zeta)-a \leq 0 \mathrm{e} \\
& \left|z_{2 s}(f, \xi, \zeta)-z_{1 s}(f, \xi, \zeta)\right|-3 a \leq 0
\end{aligned}
$$

As restrições sobre os parâmetros de projeto são

$$
\begin{aligned}
& -x_{3} \leq 0 \\
& -x_{1} \leq 0 \\
& x_{1}-2.0 \leq 0 \\
& -x_{2} \leq 0 \\
& x_{2}-0.16785 \leq 0
\end{aligned}
$$

Os dados numéricos utilizados na resolução do problema são dados a seguir: $m_{1} g=10.0 \mathrm{lb}$, $m_{2} g=1.0 \mathrm{lb}$, e $k_{1}=102 \mathrm{lb} / \mathrm{in}$. Obtem-se então $\mu=0.1$ e $\Omega_{n}=62.78$.

O exemplo foi resolvido para dois valores iniciais das variáveis de projeto, o caso 1 que corresponde um valor inicial de $\boldsymbol{x}$ contido na região viável e o caso 2 que corresponde a um valor de $\boldsymbol{x}$ fora da região viável. Um número de 100 subdivisões nas tabelas 5.43 à $5.45 \mathrm{e}$ 50 subdivisões nas tabelas 5.46 à 5.48 foram adotadas na discretização do intervalo de tempo $[0,0.667] s$.

Tabela 5.43 - Valores finais das variáveis de projeto para diferentes valores de $\boldsymbol{x}_{\mathrm{o}}$. 


\begin{tabular}{|c|c|c|c|c|c|c|c|c|}
\cline { 3 - 9 } \multicolumn{2}{c|}{} & \multicolumn{9}{c|}{ Xf } \\
\cline { 3 - 9 } \multicolumn{2}{c|}{} & $\mathbf{X o}$ & Newm/Splin & Newm/Trap & Rk4/Splin & Rk4/Trap & Rk5/Splin & Rk5/Trap \\
\hline \multirow{3}{*}{ CASO 1 1 } & $\mathbf{X 1}$ & 1,600 & 1,404 & 1,402 & 1,489 & 1,489 & 1,366 & 1,381 \\
\cline { 2 - 9 } & $\mathbf{X 2}$ & 0,020 & 0,050 & 0,051 & 0,014 & 0,014 & 0,080 & 0,058 \\
\cline { 2 - 9 } & $\mathbf{X 3}$ & 3,189 & 2,338 & 2,337 & 2,299 & 2,299 & 2,414 & 2,439 \\
\hline \multirow{3}{*}{ CASO 2 } & $\mathbf{X 1}$ & 0,000 & 1,424 & 1,404 & 1,404 & 1,404 & 1,386 & 1,386 \\
\cline { 2 - 9 } & $\mathbf{X 2}$ & 0,000 & 0,027 & 0,049 & 0,043 & 0,043 & 0,057 & 0,057 \\
\cline { 2 - 9 } & $\mathbf{X 3}$ & 0,000 & 2,359 & 2,337 & 2,298 & 2,298 & 2,411 & 2,410 \\
\hline
\end{tabular}

Tabela 5.44 - Valores finais da função objetivo para diferentes valores de $\boldsymbol{x}_{0}$.

\begin{tabular}{|l|c|c|c|c|c|c|c|}
\cline { 3 - 8 } \multicolumn{1}{c|}{} & \multicolumn{6}{c|}{$\mathbf{f}(\mathbf{X f )}$} \\
\cline { 2 - 8 } \multicolumn{1}{c|}{} & $\mathbf{f ( X o )}$ & Newm/Splin & Newm/Trap & Rk4/Splin & Rk4/Trap & Rk5/Splin & Rk5/Trap \\
\hline CASO 1 & 3,189 & 2,338 & 2,337 & 2,299 & 2,299 & 2,414 & 2,439 \\
\hline CASO 2 & 0,000 & 2,359 & 2,337 & 2,298 & 2,298 & 2,411 & 2,410 \\
\hline
\end{tabular}

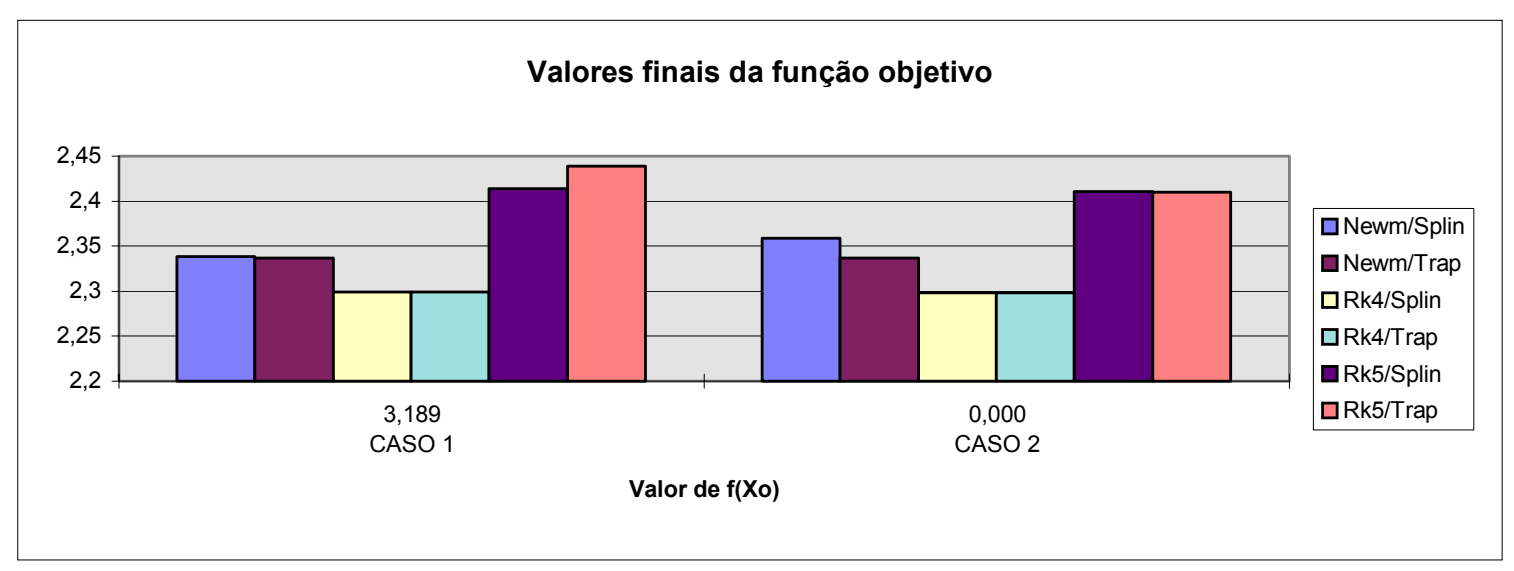

Gráfico 5.36 - Valores finais da função objetivo para diferentes valores de $\boldsymbol{x}_{\mathrm{o}}$ (tabela 5.44).

O gráfico acima mostra que o valor incial de $\boldsymbol{x}$ pouco interfere no valor final de $f(\boldsymbol{x})$. As combinações dos métodos com splines cúbicos ou com trapézio apresentam valores semelhantes para a função custo.

Tabela 5.45 - Número de minimizações sem restrições para diferentes valores de $\boldsymbol{x}_{0}$.

\begin{tabular}{|c|c|c|c|c|c|c|}
\cline { 2 - 7 } \multicolumn{1}{c|}{} & \multicolumn{7}{c|}{ K } \\
\cline { 2 - 7 } \multicolumn{1}{c|}{} & Newm/Splin & Newm/Trap & Rk4/Splin & Rk4/Trap & Rk5/Splin & Rk5/Trap \\
\hline CASO 1 & 12 & 11 & 9 & 8 & 12 & 11 \\
\hline CASO 2 & 35 & 16 & 25 & 25 & 26 & 32 \\
\hline
\end{tabular}


O gráfico 5.37 nos mostra uma sensibilidade do número de minimizações sem restrições ao valor inicial das variáveis de projeto. $\mathrm{O}$ valor incial de $\boldsymbol{x}$ no caso 2 apresenta um número maior de minimizações sem restrições do que no caso 1.

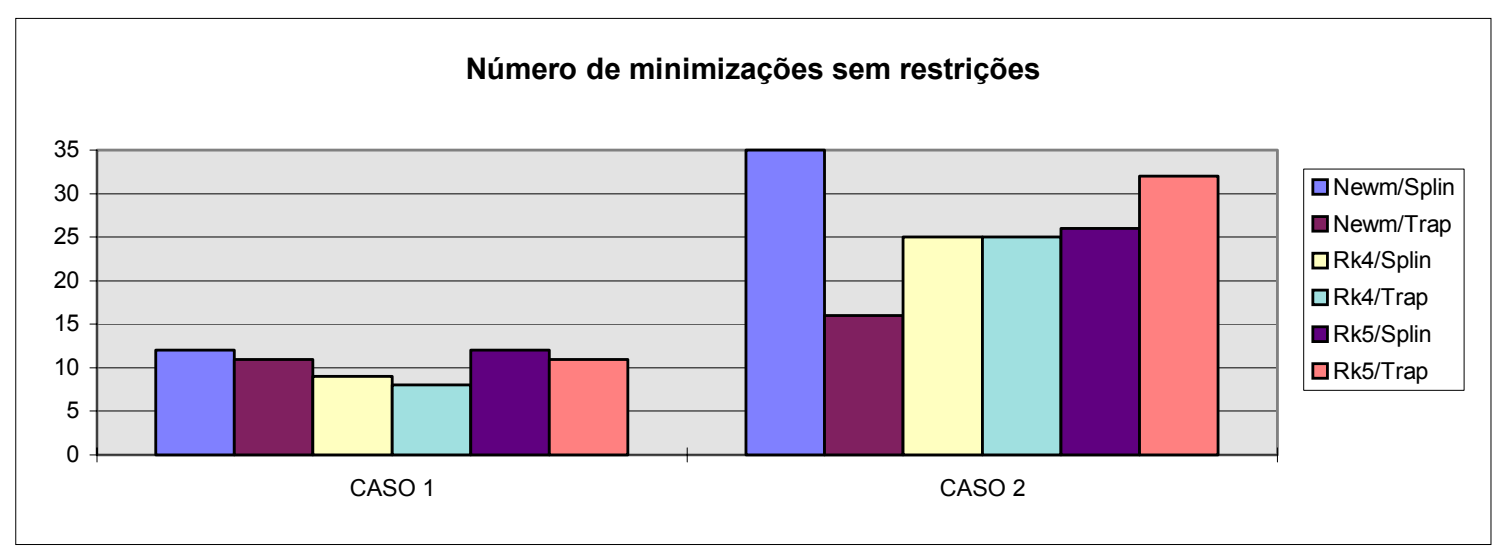

Gráfico 5.37 - Número de minimizações sem restrições para diferentes valores de $\boldsymbol{x}_{\mathrm{0}}$ (tabela 5.45).

Tabela 5.46 - Valores finais das variáveis de projeto para diferentes valores de $\boldsymbol{x}_{\mathrm{o}}$.

\begin{tabular}{|c|c|c|c|c|c|c|c|c|}
\cline { 3 - 9 } \multicolumn{2}{c|}{} & \multicolumn{8}{c|}{ Xf } \\
\cline { 3 - 9 } \multicolumn{2}{c|}{} & $\mathbf{X o}$ & Newm/Splin & Newm/Trap & Rk4/Splin & Rk4/Trap & Rk5/Splin & Rk5/Trap \\
\hline \multirow{3}{*}{ CASO 1 } & $\mathbf{X 1}$ & 1,600 & 1,446 & 1,447 & 1,489 & 1,489 & 1,344 & 1,348 \\
\cline { 2 - 9 } & $\mathbf{X 2}$ & 0,020 & 0,082 & 0,080 & 0,014 & 0,014 & 0,111 & 0,110 \\
\cline { 2 - 9 } & $\mathbf{X 3}$ & 3,189 & 1,853 & 1,853 & 2,999 & 2,299 & 2,220 & 2,218 \\
\hline \multirow{3}{*}{ CASO 2 2} & $\mathbf{X 1}$ & 0,000 & 1,427 & 1,425 & 1,404 & 1,404 & 1,354 & 1,370 \\
\cline { 2 - 9 } & $\mathbf{X 2}$ & 0,000 & 0,030 & 0,059 & 0,043 & 0,043 & 0,053 & 0,065 \\
\cline { 2 - 9 } & $\mathbf{X 3}$ & 0,000 & 2,183 & 2,075 & 2,298 & 2,298 & 2,697 & 2,486 \\
\hline
\end{tabular}

Tabela 5.47 - Valores finais da função objetivo para diferentes valores de $\boldsymbol{x}_{0}$.

\begin{tabular}{|c|c|c|c|c|c|c|c|}
\cline { 2 - 8 } \multicolumn{1}{c|}{} & $\mathbf{f ( X o )}$ & Newm/Splin & Newm/Trap & Rk4/Splin & Rk4/Trap & Rk5/Splin & Rk5/Trap \\
\cline { 2 - 8 } \multicolumn{1}{c|}{} & 3,189 & 1,853 & 1,853 & 2,999 & 2,299 & 2,220 & 2,218 \\
\hline CASO 1 & 0,000 & 2,183 & 2,075 & 2,298 & 2,298 & 2,697 & 2,486 \\
\hline
\end{tabular}

O gráfico 5.38 mostra que as combinações com a regra do trapézio apresentam menores valores da função objetivo do que as combinações com splines cúbicos. 


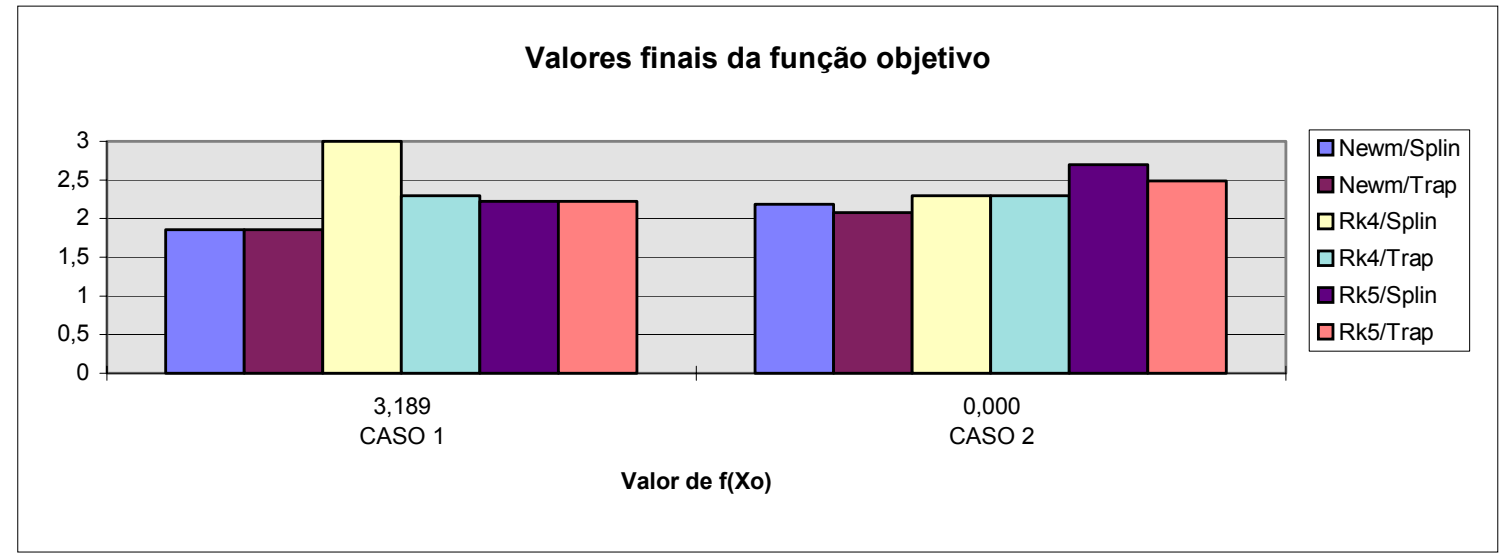

Gráfico 5.38 - Valores finais da função objetivo para diferentes valores de $\boldsymbol{x}_{\mathrm{o}}$ (tabela 5.47).

Novamente nos gráficos 5.38 e 5.39 observa-se que o valor inicial de x pouco interfere no valor de $\mathrm{f}(\mathrm{x})$, porém um valor incial "ruim" (caso 2) produz um número maior de minimizações sem restrições no Lagrangeanao Aumentado.

Tabela 5.48 - Número de minimizações sem restrições para diferentes valores de $\boldsymbol{x}_{0}$.

\begin{tabular}{|c|c|c|c|c|c|c|}
\cline { 2 - 7 } \multicolumn{1}{c|}{} & \multicolumn{7}{c|}{ K } \\
\cline { 2 - 7 } \multicolumn{1}{c|}{} & Newm/Splin & Newm/Trap & Rk4/Splin & Rk4/Trap & Rk5/Splin & Rk5/Trap \\
\hline CASO 1 & 10 & 10 & 9 & 8 & 12 & 11 \\
\hline CASO 2 & 23 & 20 & 25 & 25 & 26 & 36 \\
\hline
\end{tabular}

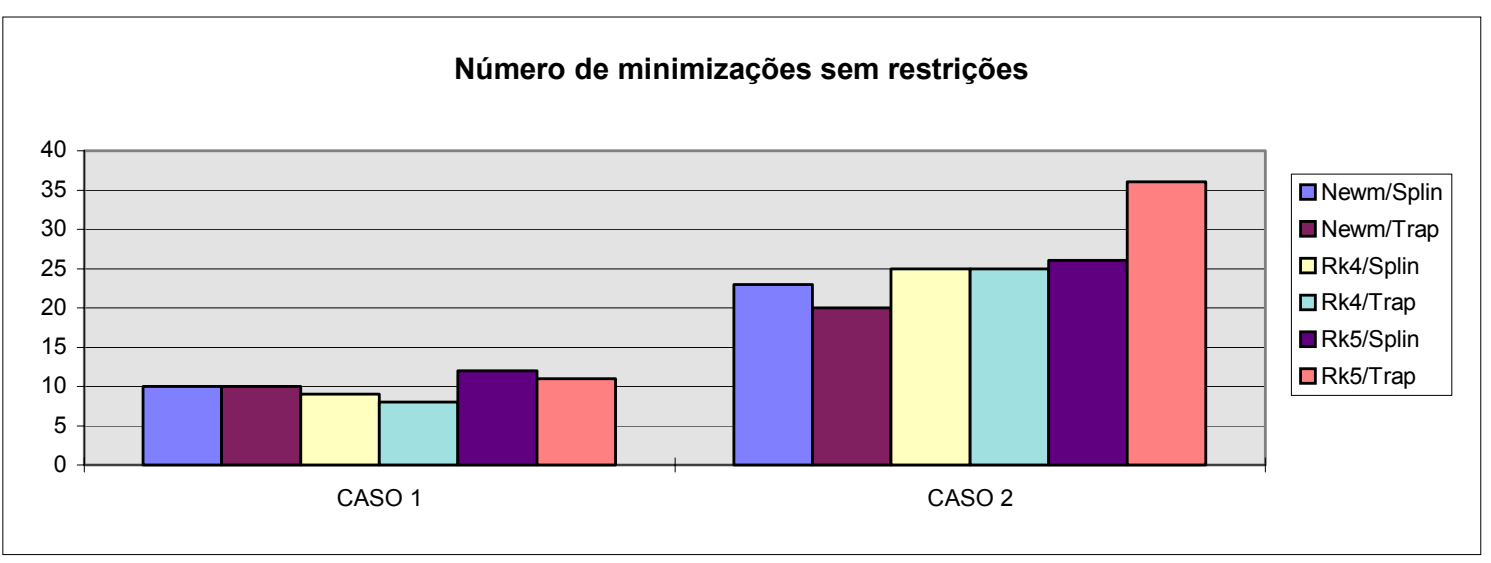

Gráfico 5.39 - Número de minimizações sem restrições para diferentes valores de $\boldsymbol{x}_{\mathrm{o}}$ (tabela 5.48). 


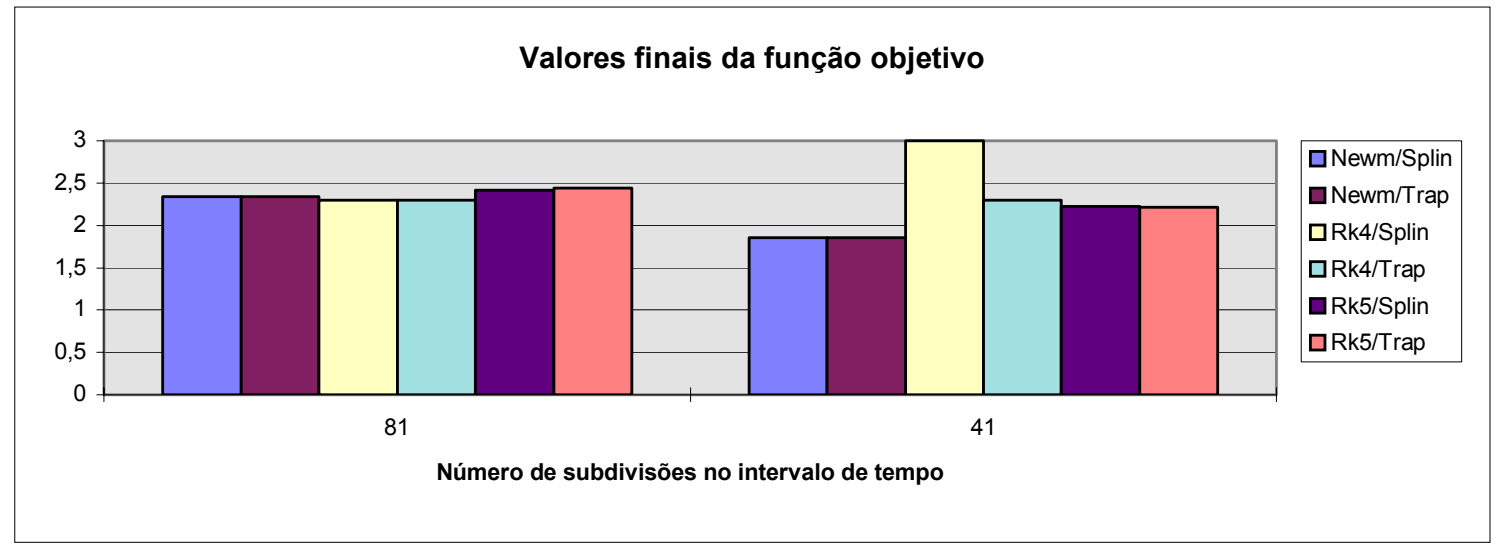

Gráfico 5.40 - Valores finiais da função objetivo no caso 1 para diferentes valores de $n$.

Observa-se uma pequena variação no valor final da função objetivo em relação à variação do número de subdivisões no intervalo de tempo. 
5.6 EXEMPLO 6 (Haug e Arora [7])

\section{Sistema de suspensão de veículo}

O dimensionamento de sistemas mecânicos em regime transiente ocorre também no campo de projeto de suspensão de veículos. Considere por exemplo o modelo de veículo com cinco graus de liberdade da figura 5.7

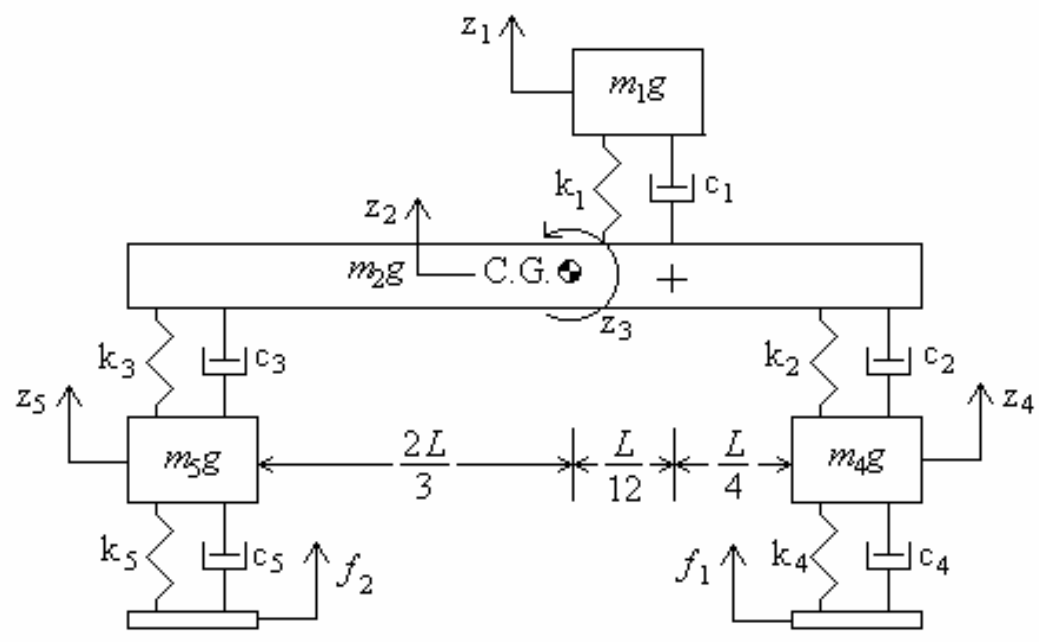

Figura 5.7 - Modelo de veículo com cinco graus de liberdade

O sistema de suspensão deve ser dimensionado de forma a minimizar o valor máximo da aceleração do banco do motorista, para uma determinada velocidade do veículo e superfície de rolamento. Restrições sobre as variáveis de estado e de projeto precisam ser impostas. As variáveis de projeto são as constantes elásticas e do amortecimento viscoso, enquanto que as de estado são os deslocamentos dos graus de liberdade.

$\mathrm{Na}$ figura acima, $m_{1}$ representa a soma das massas do assento e do motorista. $\mathrm{O}$ assento possui elasticidade $k_{1}$ e viscosidade $c_{1}$. A estrutura principal do veículo, denominado de chassis, possui massa $m_{2}$, comprimento $L$ e momento de inércia $I$ em relação ao seu centro de gravidade, e é suportada por um sistema de suspensão de elasticidades $k_{2}$ e $k_{3}$, e amortecimentos $c_{2}$ e $c_{3}$. As massas do sistema de suspensão são $m_{3}$ e $m_{4}$. Os pneus do automóvel possuem rigidezes $k_{4}$ e $k_{5}$ e amortecimentos $c_{4}$ e $c_{5}$, considerados constantes durante a análise. As funções $f_{1}(t)$ e $f_{2}(t)$ representam respectivamente os perfis da superfície 
de rolamento sob o pneus dianteiro e traseiro. O vetor das variáveis de projeto é $\boldsymbol{x}=\left[\begin{array}{lllllll}x_{1} & x_{2} & x_{3} & x_{4} & x_{5} & x_{6} & x_{7}\end{array}\right]^{T} \equiv\left[\begin{array}{lllllll}k_{1} & k_{2} & k_{3} & c_{1} & c_{2} & c_{3} & x_{7}\end{array}\right]^{T}$, sendo que $x_{7}$ representa o valor máximo da aceleração do banco do motorista.

As equações de estado para o modelo da figura 5.7 são

$$
\begin{aligned}
& {\left[\begin{array}{ccccc}
m_{1} & 0 & 0 & 0 & 0 \\
& m_{2} & 0 & 0 & 0 \\
& & I & 0 & 0 \\
& & & m_{4} & 0 \\
& & & & m_{5}
\end{array}\right]\left[\begin{array}{l}
\ddot{z}_{1} \\
\ddot{z}_{2} \\
\ddot{z}_{3} \\
\ddot{z}_{4} \\
\ddot{z}_{5}
\end{array}\right]+\left[\begin{array}{ccccc}
c_{1} & -c_{1} & -(L / 12) c_{1} & 0 & 0 \\
& c_{1}+c_{2}+c_{3} & L\left(c_{1} / 12+c_{2} / 3-2 c_{3} / 3\right) & -c_{2} & -c_{2} \\
& & L^{2}\left(c_{1} / 144+c_{2} / 9+4 c_{3} / 9\right) & -(L / 3) c_{2} & (2 L / 3) c_{3} \\
& & & c_{2}+c_{4} & 0 \\
& & & c_{3}+c_{5}
\end{array}\right]\left[\begin{array}{l}
\dot{z}_{1} \\
\dot{z}_{2} \\
\dot{z}_{3} \\
\dot{z}_{4} \\
\dot{z}_{5}
\end{array}\right]} \\
& +\left[\begin{array}{ccccc}
k_{1} & -k_{1} & -(L / 12) k_{1} & 0 & 0 \\
& k_{1}+k_{2}+k_{3} & L\left(k_{1} / 12+k_{2} / 3-2 k_{3} / 3\right) & -k_{2} & -k_{2} \\
& & L^{2}\left(k_{1} / 144+k_{2} / 9+4 k_{3} / 9\right) & -(L / 3) k_{2} & (2 L / 3) k_{3} \\
& & & k_{2}+k_{4} & 0 \\
& & & & k_{3}+k_{5}
\end{array}\right]\left[\begin{array}{c}
z_{1} \\
z_{2} \\
z_{3} \\
z_{4} \\
z_{5}
\end{array}\right]=\left[\begin{array}{c}
0 \\
0 \\
0 \\
k_{4} f_{1}(t)+c_{4} \dot{f}_{1}(t) \\
k_{5} f_{2}(t)+c_{5} \dot{f}_{2}(t)
\end{array}\right]
\end{aligned}
$$

para todo $t \in[0, \tau]$, com condições iniciais nulas. Usando a representação matricial, a equação acima pode ser colocada resumidamente na forma

$$
\boldsymbol{M} \ddot{\boldsymbol{z}}+\boldsymbol{C} \dot{\boldsymbol{z}}+\boldsymbol{K} \boldsymbol{z}=\boldsymbol{p}(t) \text { para todo } t \in[0, \tau]
$$

Pelo fato das matrizes $\boldsymbol{M}, \boldsymbol{C}$ e $\boldsymbol{K}$ serem simétricas na equação (5.33) são mostrados apenas os elementos acima da diagonal principal destas matrizes.

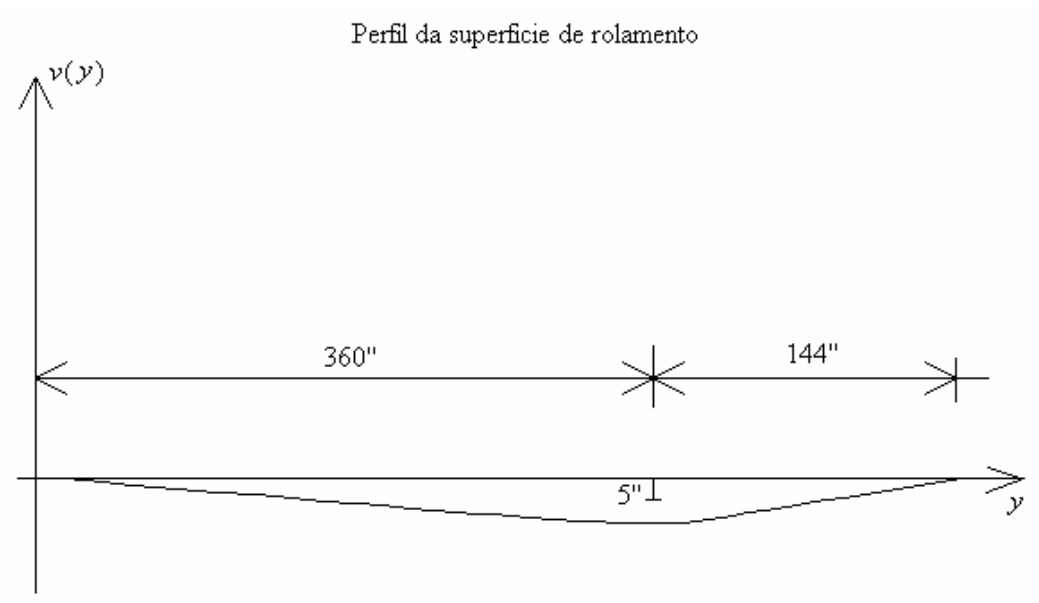

Figura 5.8 - Perfil da superfície de rolamento em função do deslocamento horizontal $y$. 
Considerando-se que a velocidade do deslocamento horizontal do veículo é igual à $450 \mathrm{in} / \mathrm{s}$ e que a superfície de rolamento mostrada na figura 5.8 possui amplitude igual a 5 in, a função $f_{1}(t)$, perfil da superfície de rolamento sob a roda dianteira, é definida como

$$
f_{1}(t)=\left\{\begin{array}{l}
5.0[1-\cos (1.25 \pi t)], \text { se } t \in[0,0.8] \\
5.0\{1+\cos [3.125 \pi(t-0.8)]\}, \text { se } t \in[0.8,1.2] \\
0, \quad \text { se } t \in[1.2, \tau]
\end{array}\right.
$$

O função $f_{2}(t)$, perfil da superfície de rolamento sob a roda traseira, deve assumir os mesmos valores que os sob a roda dianteira, porém defasado de $0.2667 \mathrm{~s}$ (tempo necessário para que a roda traseira se desloque horizontalmente de $L=120$ in - comprimento do chassis). Desta forma tem-se

$$
f_{2}(t)=\left\{\begin{array}{l}
0, \quad \text { para } t \in[0,0.2667] \\
f_{1}(t-0.2667), \quad \text { para } t \in[0.2667, \tau] .
\end{array}\right.
$$

O movimento do veículo é restringido de tal forma que os deslocamentos relativos entre o centro de gravidade (CG) do chassis e o assento do motorista (equação 5.39), o CG do chassis e o sistema de suspensão (equações 5.40 e 5.41), e o sistema de suspensão e a superfície de rolamento (equações 5.42 e 5.43), permaneçam dentro de limites determinados pelo projetista.

O objetivo é minimizar a máxima aceleração do assento do motorista, representada pela restrição 5.37. O problema, pode ser apresentado como segue:

Miniminar a função objetivo

$$
f(\boldsymbol{x})=x_{7},
$$

sujeito às restrições dinâmicas: para todo $t \in[0, \tau]$

$$
\begin{aligned}
& \left|\ddot{z}_{1}(t)\right|-x_{7} \leq 0 \\
& \left|\ddot{z}_{1}(t)\right|-400 \leq 0 \\
& \left|z_{2}(t)+(L / 12) z_{3}(t)-z_{1}(t)\right|-2 \leq 0 \\
& \left|z_{4}(t)-z_{2}(t)-(L / 3) z_{3}(t)\right|-5 \leq 0
\end{aligned}
$$




$$
\begin{aligned}
& \left|z_{5}(t)-z_{2}(t)+(2 L / 3) z_{3}(t)\right|-5 \leq 0 \\
& \left|z_{4}(t)-f_{1}(t)\right|-2 \leq 0 \\
& \left|z_{5}(t)-f_{2}(t)\right|-2 \leq 0
\end{aligned}
$$

Valores limites são impostos às variáveis de projeto:

$$
-x_{i}+x_{l i} \leq 0, i=1, \ldots, 7
$$

e

$$
x_{i}-x_{u i} \leq 0 . i=1, \ldots, 7 \text {. }
$$

Outros dados numéricos fixados durante o processo de cálculo são $m_{1} g=290 \mathrm{lb}, m_{2} g=$ $4500 \mathrm{lb}, m_{4} g=m_{5} g=96.6 \mathrm{lb}, I=41.000 \mathrm{lb} \cdot \mathrm{in}_{\mathrm{sec}} \mathrm{se}^{2}, k_{4}=k_{5}=1500 \mathrm{lb} / \mathrm{in}, \mathrm{e} c_{4}=c_{5}=5 \mathrm{lb} . \mathrm{s} / \mathrm{in}$. $\mathrm{O}$ valores máximos e mínimos assumidos pelas variáveis de projeto são $\boldsymbol{x}_{l}=\left[\begin{array}{lllllll}50 & 200 & 200 & 2 & 5 & 5 & 1\end{array}\right]^{T} \quad \mathrm{e} \quad \boldsymbol{x}_{u}=\left[\begin{array}{llllllll}500 & 1000 & 1000 & 50 & 80 & 80 & 500\end{array}\right]^{T}$ respectivamente. A unidade dos descolamentos $z_{1}, z_{2}, z_{4}$ e $z_{5}$ é a polegada (in), do deslocamento $\mathrm{z}_{3}$ é radiano, e do tempo segundo $(s)$. O valor de $\tau=2.24 s$.

O exemplo foi resolvido para dois valores iniciais das variáveis de projeto, o caso 1 que corresponde um valor inicial de $\boldsymbol{x}$ contido na região viável e o caso 2 que corresponde a um valor de $\boldsymbol{x}$ fora da região viável. Um número de 100 subdivisões nas tabelas 5.49 à $5.51 \mathrm{e}$ 50 subdivisões nas tabelas 5.52 à 5.54 foram adotadas na discretização do intervalo de tempo $[0,0.667] s$.

Tabela 5.49 - Valores finais das variáveis de projeto para diferentes valores de $\boldsymbol{x}_{0}$.

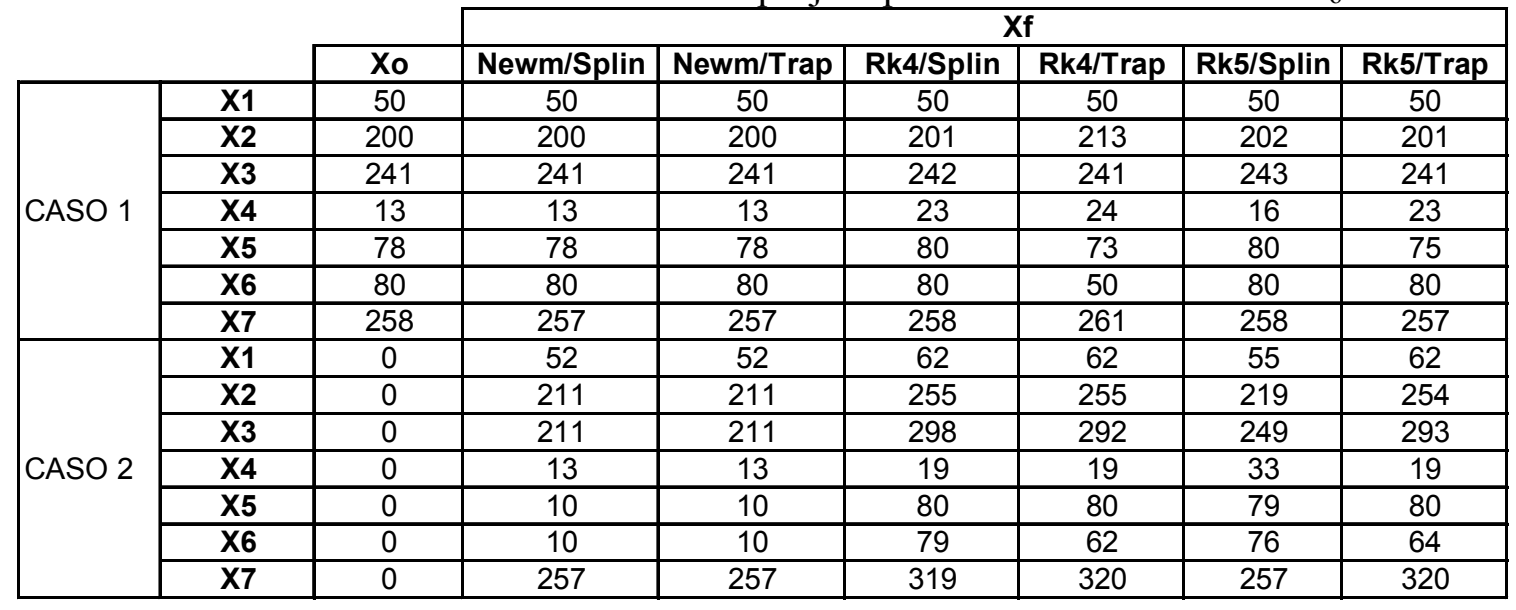

Tabela 5.50 - Valores finais da função objetivo para diferentes valores de $\boldsymbol{x}_{\mathrm{0}}$. 


\begin{tabular}{|c|c|c|c|c|c|c|c|}
\cline { 3 - 8 } \multicolumn{1}{c|}{} & \multicolumn{6}{c|}{$\mathbf{f}(\mathbf{X f})$} \\
\cline { 2 - 8 } \multicolumn{1}{c|}{} & $\mathbf{f}(\mathbf{X o})$ & Newm/Splin & Newm/Trap & Rk4/Splin & Rk4/Trap & Rk5/Splin & Rk5/Trap \\
\hline CASO 1 & 258,000 & 257,000 & 257,000 & 258,000 & 261,000 & 258,000 & 257,000 \\
\hline CASO 2 & 0,000 & 257,000 & 257,000 & 319,000 & 320,000 & 257,000 & 320,000 \\
\hline
\end{tabular}

O gráfico 5.41 mostra uma pequena sensibilidade do método dos Multiplicadores em relação ao valor inicial de $\boldsymbol{x}$. Observa-se também que as combinações com o método de Newmark apresentaram praticamente os mesmos valores para a $f(\boldsymbol{x})$. Este fato mostra que os valores da função objetivo apresentados por estas combinações são mais estáveis em relação à variação de $\boldsymbol{x}_{0}$.

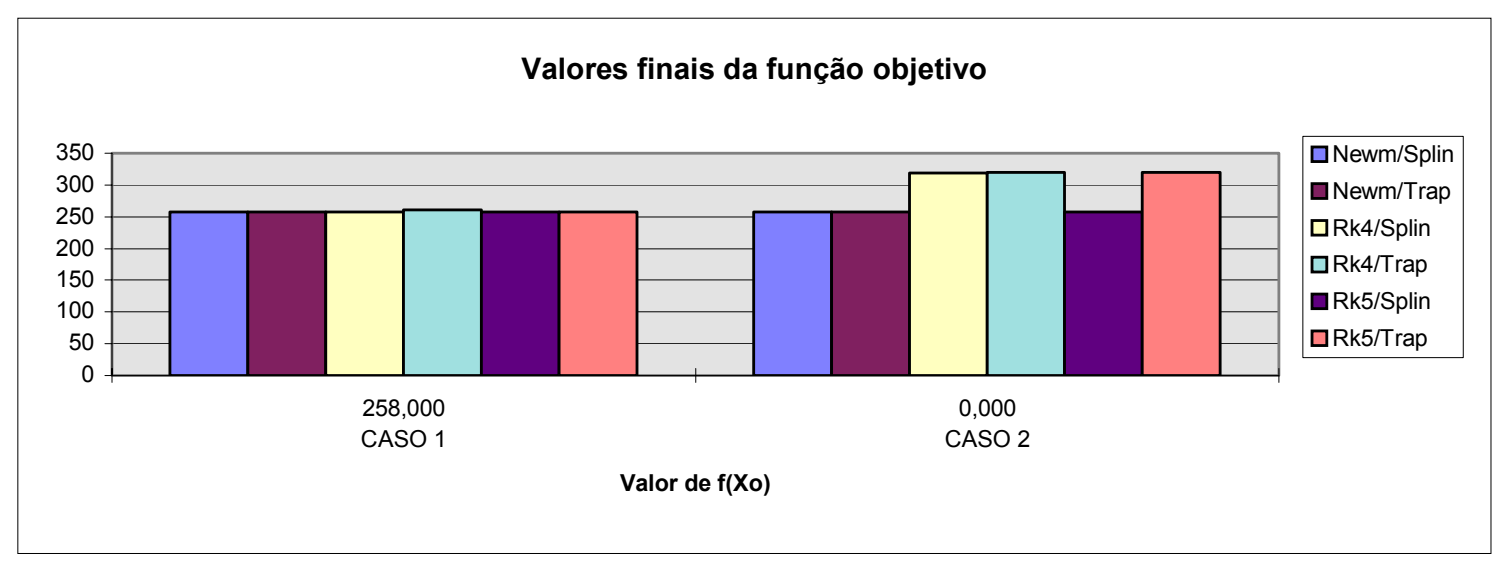

Gráfico 5.41 - Valores finais da função objetivo para diferentes valores de $\boldsymbol{x}_{\mathrm{o}}$ (tabela 5.50).

Tabela 5.51 - Número de minimizações sem restrições para diferentes valores de $\boldsymbol{x}_{0}$.

\begin{tabular}{|l|c|c|c|c|c|c|}
\cline { 2 - 7 } \multicolumn{1}{c|}{} & \multicolumn{6}{c|}{ K } \\
\cline { 2 - 7 } \multicolumn{1}{c|}{} & Newm/Splin & Newm/Trap & Rk4/Splin & Rk4/Trap & Rk5/Splin & Rk5/Trap \\
\hline CASO 1 & 11 & 10 & 11 & 15 & 13 & 14 \\
\hline CASO 2 & 21 & 21 & 14 & 15 & 23 & 14 \\
\hline
\end{tabular}

O gráfico 5.42 mostra que o número de minimizações sem restrições foram maiores no caso 2 do que no caso 1 . 


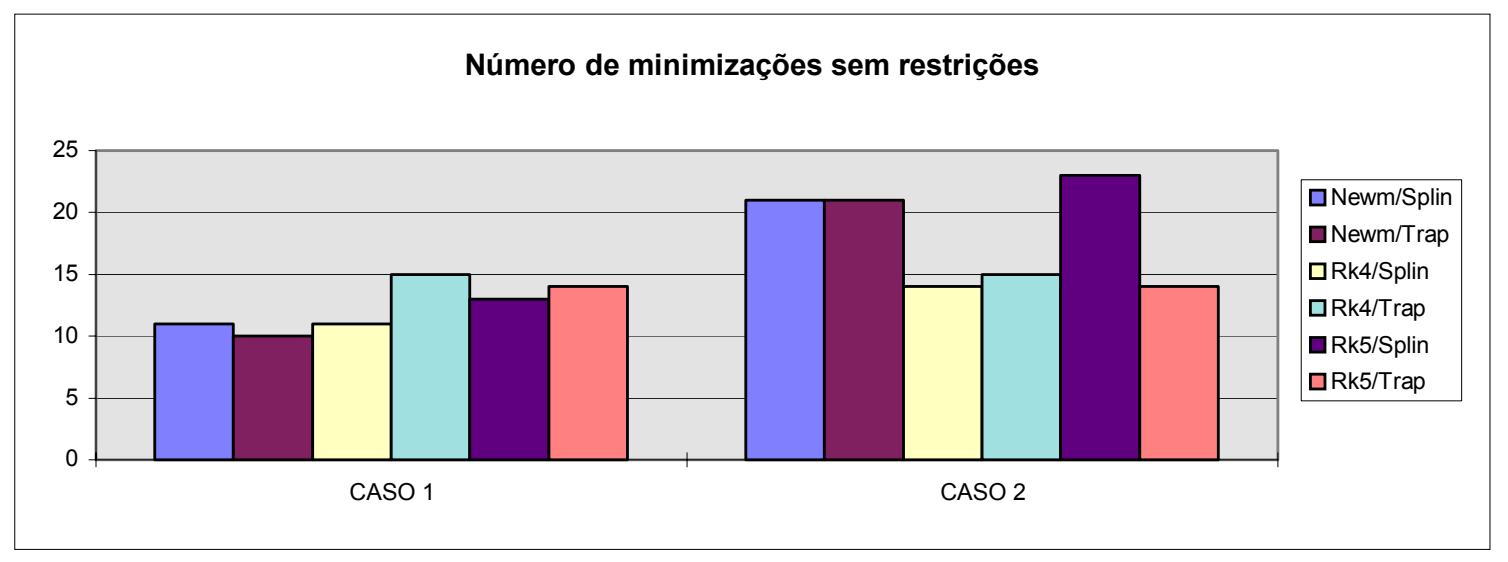

Gráfico 5.42 - Número de minimizações sem restrições para diferentes valores de $\boldsymbol{x}_{\mathrm{o}}$ (tabela 5.51).

Tabela 5.52 - Valores finais das variáveis de projeto para diferentes valores de $\boldsymbol{x}_{0}$.

\begin{tabular}{|c|c|c|c|c|c|c|c|c|}
\hline & & \multicolumn{6}{|c|}{$\overline{X f}$} \\
\hline & & Xo & Newm/Splin & Newm/Trap & Rk4/Splin & Rk4/Trap & Rk5/Splin & Rk5/Trap \\
\hline \multirow{7}{*}{ CASO 1} & $\overline{X 1}$ & 50 & 50 & 50 & 50 & 51 & 50 & 50 \\
\hline & $\overline{X 2}$ & 200 & 200 & 200 & 238 & 228 & 202 & 201 \\
\hline & $\overline{X 3}$ & 241 & 241 & 241 & 275 & 242 & 243 & 241 \\
\hline & $\mathrm{X4}$ & 13 & 13 & 13 & 23 & 24 & 16 & 23 \\
\hline & $\overline{X 5}$ & 78 & 78 & 78 & 80 & 78 & 80 & 77 \\
\hline & $\mathrm{X6}$ & 80 & 80 & 80 & 80 & 50 & 80 & 80 \\
\hline & $\mathrm{X7}$ & 258 & 257 & 257 & 258 & 258 & 258 & 257 \\
\hline \multirow{7}{*}{ CASO 2} & $\overline{X 1}$ & 0 & 52 & 52 & 62 & 62 & 55 & 62 \\
\hline & $\overline{X 2}$ & 0 & 211 & 211 & 254 & 254 & 219 & 254 \\
\hline & $\overline{X 3}$ & 0 & 211 & 211 & 298 & 292 & 249 & 292 \\
\hline & $\overline{X 4}$ & 0 & 13 & 13 & 19 & 19 & 33 & 19 \\
\hline & $\overline{X 5}$ & 0 & 10 & 10 & 80 & 80 & 79 & 80 \\
\hline & $\overline{X 6}$ & 0 & 10 & 10 & 79 & 62 & 76 & 64 \\
\hline & $\mathrm{X7}$ & 0 & 257 & 257 & 319 & 320 & 257 & 320 \\
\hline
\end{tabular}

Tabela 5.53 - Valores finais da função objetivo para diferentes valores de $\boldsymbol{x}_{0}$.

\begin{tabular}{|c|c|c|c|c|c|c|c|}
\cline { 3 - 8 } \multicolumn{1}{c|}{} & $\mathbf{f ( X o )}$ & Newm/Splin & Newm/Trap & Rk4/Splin & Rk4/Trap & Rk5/Splin & Rk5/Trap \\
\cline { 2 - 8 } \multicolumn{1}{c|}{} & 258,000 & 257,000 & 257,000 & 258,000 & 258,000 & 258,000 & 257,000 \\
\hline CASO 1 & 253,000 & 257,000 & 319,000 & 320,000 & 257,000 & 320,000 \\
\hline
\end{tabular}

Novamente no gráfico 5.43 se observa que os valores finais da função objetivo são pouco sensíveis ao valor inicial de $\boldsymbol{x}$. 


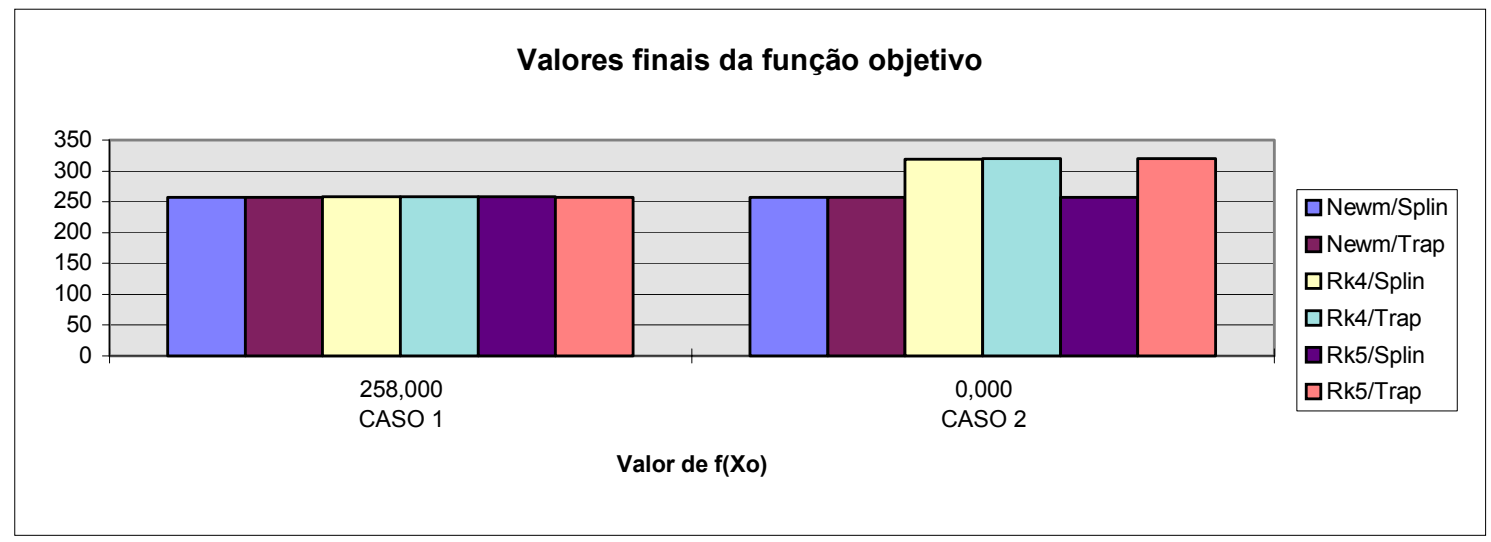

Gráfico 5.43 - Valores finais da função objetivo para diferentes valores de $\boldsymbol{x}_{\mathrm{o}}$ (tabela 5.53).

Tabela 5.54 - Número de minimizações sem restrições para diferentes valores de $\boldsymbol{x}_{\mathrm{0}}$.

\begin{tabular}{|c|c|c|c|c|c|c|}
\cline { 2 - 7 } \multicolumn{1}{c|}{} & \multicolumn{7}{c|}{ K } \\
\cline { 2 - 7 } \multicolumn{1}{c|}{} & Newm/Splin & Newm/Trap & Rk4/Splin & Rk4/Trap & Rk5/Splin & Rk5/Trap \\
\hline CASO 1 & 11 & 10 & 15 & 15 & 14 & 14 \\
\hline CASO 2 & 21 & 19 & 14 & 15 & 23 & 14 \\
\hline
\end{tabular}

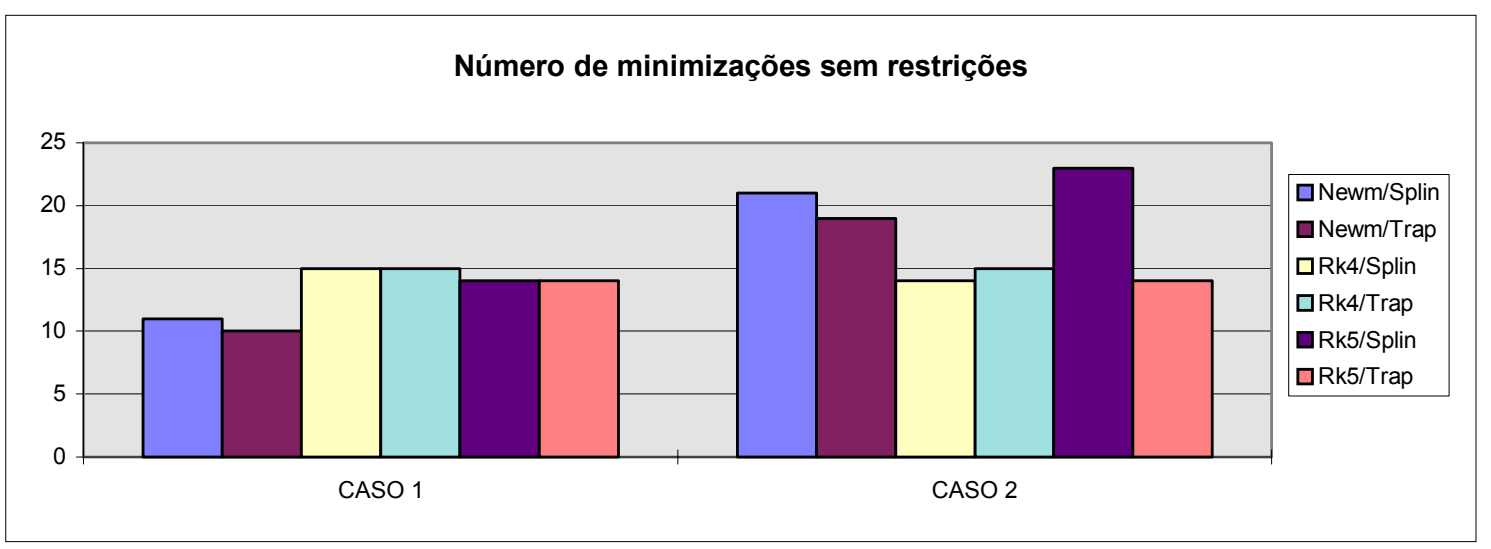

Gráfico 5.44 - Número de minimizações sem restrições para diferentes valores de $\boldsymbol{x}_{\mathrm{o}}$ (tabela 5.54).

Já o gráfico 5.44 mostra que valores de $\boldsymbol{x}_{\mathrm{o}}$ fora da região viável produz um número maior de minimizações sem restrições do que valores contidos na região viável. Ainda se observa um melhor desempenho das combinações com trapézio do que as combinações com splines cúbicos. 


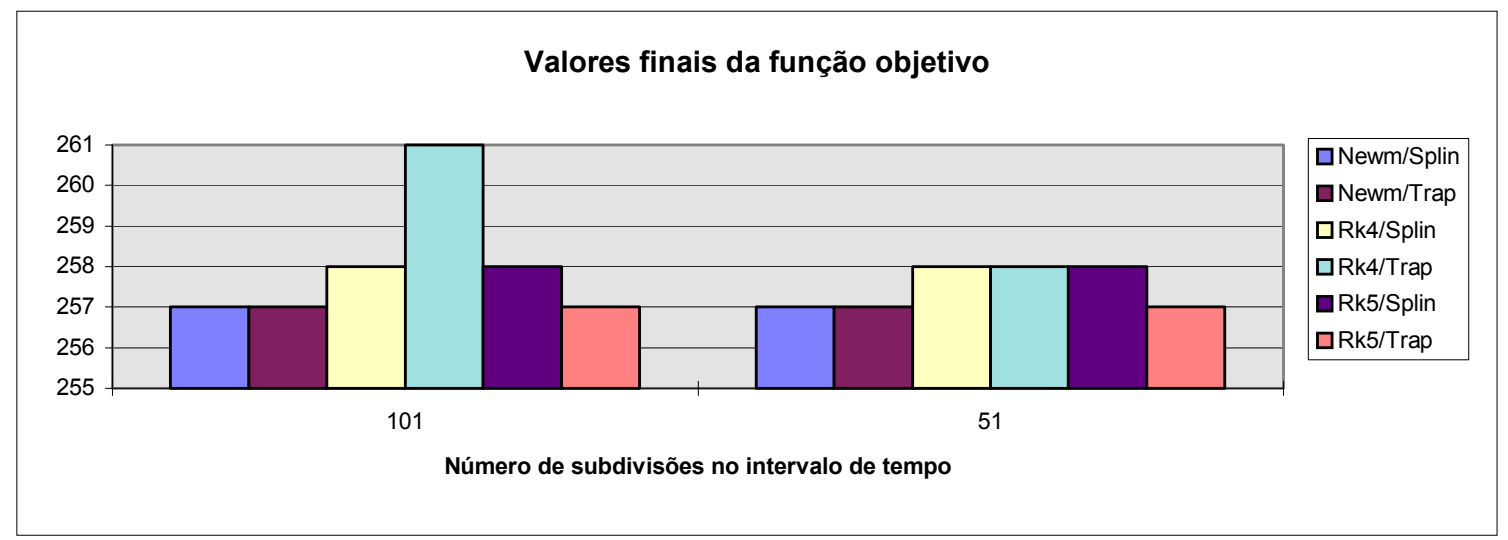

Gráfico $\mathbf{5 . 4 5}$ - Valores finais da função objetivo no caso 1 para diferentes valores de $n$.

O gráfico 5.45 mostra que as combinações com os métodos de Newmark e Runge-Kutta de quinta ordem praticamente não apresentam sensibilidade em relação à variação do número de subdivisões no intervalo de tempo. Com o método de Newmark obtém-se menores valores para a função objetivo. 


\subsection{EXEMPLO 7 (Pimenta, Goldenberg, Brasil e Silva [23])}

\section{Shear-building submetido à explosão}

O exemplo seguinte é o dimensionamento ótimo dos pilares do shear-building com dez graus de liberdade mostrado na figura 5.9a. O edifício é solicitado por uma explosão próxima aos três últimos pisos. O objetivo é minimizar o peso das colunas. As variáveis de projeto são as rigidezes de cada andar divididas pelo fator de escala 570000. Restrições são impostas às variáveis de projeto, deslocamentos e tensões segundo normas do AISC.

Os pesos dos pisos e paredes estão indicados na figura. Adimite-se que as propriedades são uniformes ao longo do prédio. Todas as colunas do andar são supostas semelhantes (mesma, área, momento de inércia, ...). O pé direito $10 \mathrm{ft}$, e a distância entre as colunas mede $20 \mathrm{ft}$. A massa concentrada em cada grau de liberdade é igual à soma das massas dos pisos e paredes, sendo um total de $816.8 \mathrm{lbs}^{2} / \mathrm{in}$. Utiliza-se o modelo elástico linear representado pelo sistema de massas da figura 5.9b. A rigidez de um andar é a soma das rigidezes de todos os pilares do andar. A constante elástica de uma coluna do $i$-ésimo andar é

$$
k_{i}=12 E I_{i} / h_{i}{ }^{3},
$$

onde $E$ é o módulo de elasticidade, $I_{i}$ o momento de inércia e $h_{i}$ o comprimento do pilar.

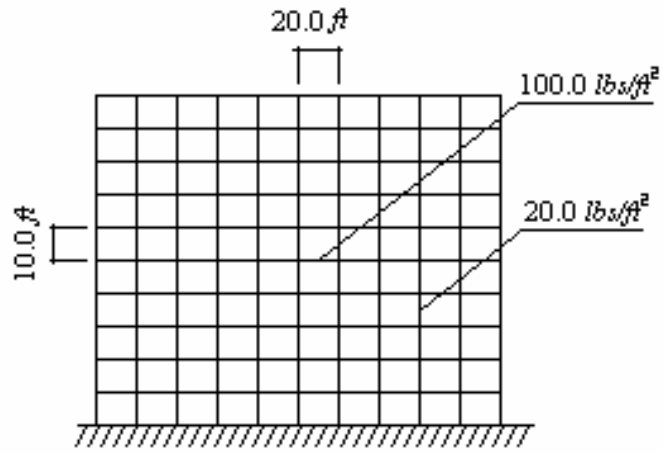

(a)

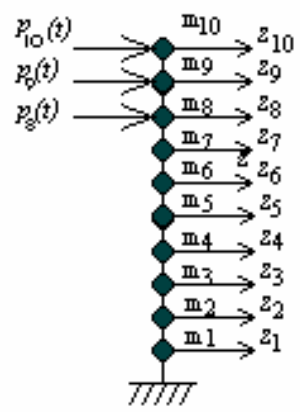

(b)

Figura 5.9 - Shear-building com dez graus de liberdade 
A equação do movimento para o modelo acima é dada de forma explícita:

$$
\left.\left[\begin{array}{ccccc}
m_{1} & 0 & \cdots & 0 & 0 \\
0 & m_{2} & \cdots & 0 & 0 \\
\vdots & \vdots & \ddots & \vdots & \vdots \\
0 & 0 & \cdots & m_{9} & 0 \\
0 & 0 & \cdots & 0 & m_{10}
\end{array}\right]\left[\begin{array}{l}
z_{1}(t) \\
z_{2}(t) \\
\vdots \\
z_{9}(t) \\
z_{10}(t)
\end{array}\right]+\left[\begin{array}{ccccc}
K_{1}+K_{2} & -K_{2} & \cdots & 0 & 0 \\
-K_{2} & K_{2}+K_{3} & \cdots & 0 & 0 \\
\vdots & \vdots & \ddots & \vdots & \vdots \\
0 & 0 & \cdots & K_{9}+K_{10} & -K_{10} \\
0 & 0 & \cdots & -K_{10} & K_{10}
\end{array}\right] \begin{array}{c}
z_{1}(t) \\
z_{2}(t) \\
\vdots \\
z_{9}(t) \\
z_{10}(t)
\end{array}\right]=\left[\begin{array}{c}
p_{1}(t) \\
p_{2}(t) \\
\vdots \\
p_{9}(t) \\
p_{10}(t)
\end{array}\right],
$$

sendo que $t \in[0,1.0] \mathrm{s}$, e as condições iniciais $\boldsymbol{z}(0)=\mathbf{0}$ e $\boldsymbol{z}(0)=\mathbf{0}$. Resumidamente, podese escrever: $\boldsymbol{M} \ddot{\boldsymbol{z}}(t)+\boldsymbol{K} \boldsymbol{z}(t)=\boldsymbol{p}(t), t \in[0,1.0]$ s com condições iniciais nulas.

O vetor força generalizada é definido por

$$
\begin{aligned}
& p_{i}(t)=0.0 l b, \quad 0 \leq t \leq 1.0 s, \quad i=1, \ldots, 7 \\
& p_{i}(t)=\left\{\begin{array}{ll}
20000\left(1.0-t / t_{d}\right) l b s, & 0 \leq t \leq t_{d} \\
0 l b, & t_{d} \leq t \leq 1.0 s
\end{array}\right\}, \quad i=8, \ldots, 10
\end{aligned}
$$

sendo que $t_{d}=0.1 \mathrm{~s}$.

Dois tipo de restrições dinâmicas são impostas sobre deslocamentos e tensões. O máximo deslocamento relativo permitido entre pisos adjacentes é 0.33333 in e a máxima tensão nos pilares $14400 \mathrm{psi}$. Os valores máximo e mínimo para as variáveis de projeto são respectivamente 100.0 e $0.0001 \mathrm{lb} / \mathrm{in}$. A densidade do aço é dada por $0.28 \mathrm{lb}_{\mathrm{in}} \mathrm{in}^{3}$.

A área da seção transversal e módulo de resistência à flexão dos pilares podem ser obtido por

$$
\begin{aligned}
& A_{i}=0.465 I_{i}^{1 / 2} \mathrm{e} \\
& S_{i}=0.544 I_{i}^{3 / 4} .
\end{aligned}
$$

Estas relações são utilizadas pelo AISC para seções de aço e independem do sistema de unidades utilizado. A rigidez do $i$-ésimo pilar é (11 colunas)

$$
K_{i}=11 k_{i} .
$$


Considerando $E=3010^{6}$ psi e $h_{i}=10 \times 12=120$ in obtem-se

$$
K_{i}=2291.6667 I_{i} .
$$

As tensões atuante nos pilares são

$$
\sigma_{1}=K_{1} z_{1} / 11 \text { e } \sigma_{i}=K_{i}\left(z_{i}-z_{i-1}\right) / 11, i=2, \ldots, 10 .
$$

O problema de programação matemática pode ser resumido por

minimizar

$$
\begin{aligned}
f(\boldsymbol{x}) & =11 \rho \sum_{i=1}^{10} A_{i} h_{i} \quad l b \\
& =11(0.28)(0.465) 120 \sum_{i=1}^{10}\left(k_{i} / 2291.6667\right)^{0.5} \\
& =11(0.28)(0.465) 120 \sum_{i=1}^{10}\left(570000 x_{i} / 2291.6667\right)^{0.5} \\
& =2710.483 \sum_{i=1}^{10} x_{i}^{0.5}
\end{aligned}
$$

Sujeito a:

1. restrições sobre deslocamento (desigualdades)

$$
\begin{aligned}
& g_{1}=\left|z_{1}(t)\right|-0.33333, \quad t \in[0,0.3] ; \\
& g_{i}=\left|z_{i}(t)-z_{i-1}(t)\right|-0.33333, \quad t \in[0,0.3] ; \quad i=2, \ldots, 10 .
\end{aligned}
$$

2. restrições sobre tensões (desigualdades)

$$
\begin{aligned}
& g_{11}=570000 x_{1}\left|z_{1}(t)\right| /\left[11 A_{i}(\boldsymbol{x})\right]-14400, \quad t \in[0,0.3] ; \\
& g_{(i+10)}=570000 x_{i}\left|z_{i}(t)-z_{i-1}(t)\right| /\left[11 A_{i}(\boldsymbol{x})\right]-14400, \quad t \in[0,0.3] ; \quad i=2, \ldots, 10 .
\end{aligned}
$$

3. valores máximos e mínimos para as variáveis de projeto

$$
0.0001 \leq x_{i} \leq 100.0, \quad i=1, \ldots, 10 \text {. }
$$

O exemplo foi resolvido para dois valores iniciais de $\boldsymbol{x}$, o caso 1 e o caso 2 . Um número de 100 subdivisões nas tabelas 5.55 à 5.57 e 50 subdivisões nas tabelas 5.58 à 5.60 foram adotadas na discretização do intervalo de tempo $[0,1.0] \mathrm{s}$. 
Tabela 5.55 - Valores finais das variáveis de projeto para diferentes valores de $\boldsymbol{x}_{0}$.

\begin{tabular}{|c|c|c|c|c|c|c|c|c|}
\hline & & \multicolumn{6}{|c|}{$\overline{X f}$} \\
\hline & & Xo & Newm/Splin & Newm/Trap & Rk4/Splin & Rk4/Trap & Rk5/Splin & Rk5/Trap \\
\hline \multirow{10}{*}{ CASO 1} & $\mathrm{X1}$ & 0,400 & 0,423 & 0,425 & 0,498 & 0,499 & 0,499 & 0,502 \\
\hline & $\mathrm{X2}$ & 0,400 & 0,409 & 0,408 & 0,432 & 0,432 & 0,432 & 0,433 \\
\hline & $\mathrm{X3}$ & 0,400 & 0,399 & 0,403 & 0,408 & 0,407 & 0,407 & 0,405 \\
\hline & $\mathrm{X4}$ & 0,400 & 0,368 & 0,368 & 0,376 & 0,375 & 0,376 & 0,374 \\
\hline & $X 5$ & 0,400 & 0,366 & 0,367 & 0,377 & 0,375 & 0,378 & 0,376 \\
\hline & $\mathrm{X6}$ & 0,400 & 0,365 & 0,366 & 0,377 & 0,374 & 0,378 & 0,378 \\
\hline & $\mathrm{X7}$ & 0,400 & 0,320 & 0,319 & 0,381 & 0,380 & 0,379 & 0,380 \\
\hline & $\mathrm{X8}$ & 0,400 & 0,368 & 0,368 & 0,392 & 0,393 & 0,393 & 0,392 \\
\hline & $X 9$ & 0,400 & 0,397 & 0,399 & 0,402 & 0,405 & 0,403 & 0,401 \\
\hline & $\mathrm{X10}$ & 0,400 & 0,401 & 0,387 & 0,413 & 0,418 & 0,415 & 0,412 \\
\hline \multirow{10}{*}{ CASO 2} & $\mathrm{X} 1$ & 0,500 & 0,425 & 0,424 & 0,480 & 0,481 & 0,482 & 0,505 \\
\hline & $\mathrm{X2}$ & 0,500 & 0,407 & 0,397 & 0,419 & 0,420 & 0,421 & 0,430 \\
\hline & $\mathrm{X3}$ & 0,500 & 0,408 & 0,401 & 0,380 & 0,380 & 0,383 & 0,399 \\
\hline & $\mathrm{X} 4$ & 0,500 & 0,369 & 0,373 & 0,380 & 0,381 & 0,382 & 0,387 \\
\hline & $X 5$ & 0,500 & 0,368 & 0,354 & 0,380 & 0,378 & 0,378 & 0,378 \\
\hline & $\mathrm{X6}$ & 0,500 & 0,330 & 0,330 & 0,378 & 0,378 & 0,379 & 0,378 \\
\hline & $\mathrm{X7}$ & 0,500 & 0,343 & 0,341 & 0,380 & 0,381 & 0,383 & 0,384 \\
\hline & $\mathrm{X8}$ & 0,500 & 0,359 & 0,342 & 0,393 & 0,392 & 0,394 & 0,394 \\
\hline & $\mathrm{X9}$ & 0,500 & 0,392 & 0,407 & 0,401 & 0,405 & 0,415 & 0,416 \\
\hline & $\mathrm{X10}$ & 0,500 & 0,406 & 0,415 & 0,412 & 0,420 & 0,419 & 0,422 \\
\hline
\end{tabular}

Tabela 5.56 - Valores finais da função objetivo para diferentes valores de $\boldsymbol{x}_{0}$.

\begin{tabular}{|l|c|c|c|c|c|c|c|}
\cline { 2 - 8 } \multicolumn{1}{c|}{} & \multicolumn{7}{c|}{$\mathbf{f ( X f )}$} \\
\cline { 2 - 8 } \multicolumn{1}{c|}{} & $\mathbf{f ( X o )}$ & Newm/Splin & Newm/Trap & Rk4/Splin & Rk4/Trap & Rk5/Splin & Rk5/Trap \\
\hline CASO 1 & 17143 & 16732 & 16719 & 17247 & 17250 & 17255 & 17239 \\
\hline CASO 2 & 19166 & 16711 & 16657 & 17138 & 17165 & 17208 & 17325 \\
\hline
\end{tabular}

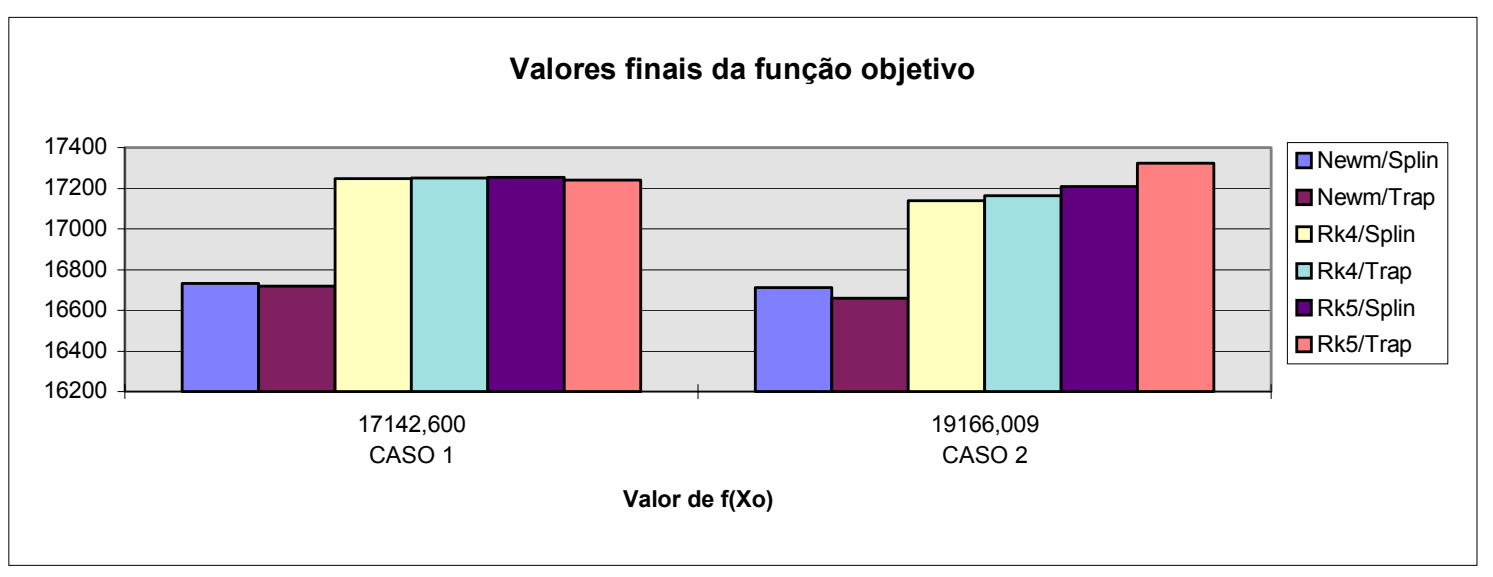

Gráfico 5.46 - Valores finais da função objetivo para diferentes valores de $\boldsymbol{x}_{\mathrm{o}}$ (tabela 5.56). 
O gráfico 5.46 mostra uma sensibilidade do Lagrangeano aumentado em relação à variação da solução da equação do movimento.

Tabela 5.57 - Número de minimizações sem restrições para diferentes valores de $\boldsymbol{x}_{0}$.

\begin{tabular}{|l|c|c|c|c|c|c|}
\cline { 2 - 7 } \multicolumn{1}{c|}{} & \multicolumn{7}{c|}{ K } \\
\cline { 2 - 7 } \multicolumn{1}{c|}{} & Newm/Splin & Newm/Trap & Rk4/Splin & Rk4/Trap & Rk5/Splin & Rk5/Trap \\
\hline CASO 1 & 26 & 24 & 31 & 28 & 27 & 26 \\
\hline CASO 2 & 25 & 23 & 30 & 28 & 28 & 26 \\
\hline
\end{tabular}

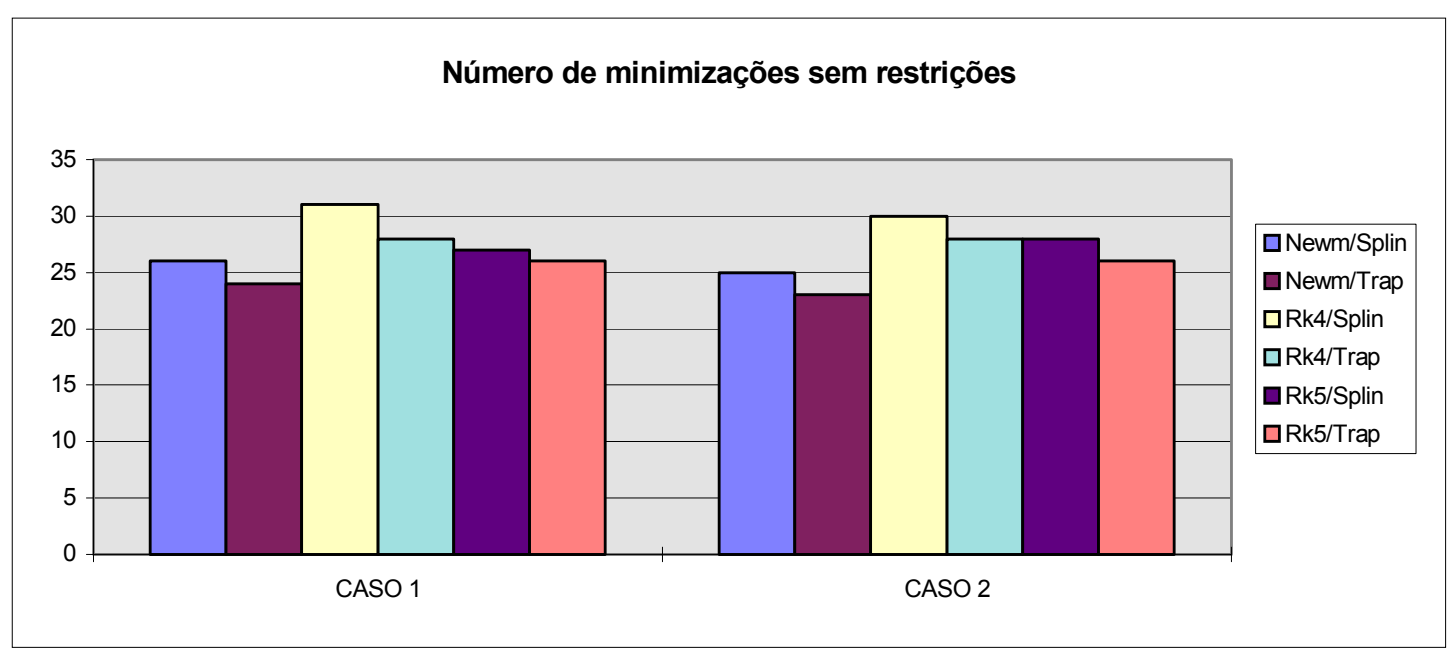

Gráfico 5.47 - Número de minimizações sem restrições para diferentes valores de $\boldsymbol{x}_{\mathrm{o}}$ (Tabela 5.57).

Tabela 5.58 - Valores finais das variáveis de projeto para diferentes valores de $\boldsymbol{x}_{\mathrm{o}}$.

\begin{tabular}{|c|c|c|c|c|c|c|c|c|}
\hline & & \multicolumn{6}{|c|}{$x+$} \\
\hline & & $X_{0}$ & Newm/Splin & Newm/Trap & Rk4/Splin & Rk4/Trap & \begin{tabular}{|l|} 
Rk5/Splin \\
\end{tabular} & Rk5/Trap \\
\hline \multirow{10}{*}{ CASO 1} & $\mathrm{X} 1$ & 0,400 & 0,452 & 0,453 & 0,501 & 0,501 & 0,500 & 0,499 \\
\hline & $\mathrm{X} 2$ & 0,400 & 0,447 & 0,447 & 0,491 & 0,494 & 0,493 & 0,496 \\
\hline & X3 & 0,400 & 0,436 & 0,436 & 0,489 & 0,493 & 0,478 & 0,481 \\
\hline & X4 & 0,400 & 0,438 & 0,435 & 0,475 & 0,474 & 0,469 & 0,477 \\
\hline & X5 & 0,400 & 0,426 & 0,429 & 0,468 & 0,470 & 0,451 & 0,469 \\
\hline & X6 & 0,400 & 0,431 & 0,430 & 0,460 & 0,468 & 0,445 & 0,458 \\
\hline & $\mathrm{X7}$ & 0,400 & 0,414 & 0,428 & 0,457 & 0,452 & 0,453 & 0,453 \\
\hline & $\mathbf{X 8}$ & 0,400 & 0,419 & 0,420 & 0,458 & 0,457 & 0,454 & 0,453 \\
\hline & $\begin{array}{l}\mathbf{X 9} \\
\end{array}$ & 0,400 & 0,427 & 0,425 & 0,465 & 0,468 & 0,461 & 0,465 \\
\hline & $\mathbf{X 1 0}$ & 0,400 & 0,435 & 0,431 & 0,472 & 0,476 & 0,467 & 0,472 \\
\hline \multirow{10}{*}{ CASO 2} & $\mathrm{X} 1$ & 0,500 & 0,467 & 0,458 & 0,503 & 0,502 & 0,499 & 0,501 \\
\hline & $\mathrm{X} 2$ & 0,500 & 0,451 & 0,453 & 0,493 & 0,500 & 0,492 & 0,489 \\
\hline & X3 & 0,500 & 0,428 & 0,435 & 0,490 & 0,496 & 0,475 & 0,477 \\
\hline & X4 & 0,500 & 0,429 & 0,428 & 0,488 & 0,489 & 0,461 & 0,463 \\
\hline & $\mathrm{X5}$ & 0,500 & 0,433 & 0,430 & 0,485 & 0,485 & 0,432 & 0,473 \\
\hline & X6 & 0,500 & 0,436 & 0,420 & 0,490 & 0,497 & 0,443 & 0,459 \\
\hline & $\mathrm{X7}$ & 0,500 & 0,435 & 0,441 & 0,492 & 0,501 & 0,457 & 0,463 \\
\hline & $\mathbf{X 8}$ & 0,500 & 0,437 & 0,444 & 0,496 & 0,501 & 0,481 & 0,478 \\
\hline & X9 & 0,500 & 0,438 & 0,449 & 0,501 & 0,503 & 0,484 & 0,485 \\
\hline & $\mathbf{X 1 0}$ & 0,500 & 0,440 & 0,449 & 0,502 & 0,505 & 0,486 & 0,494 \\
\hline
\end{tabular}


Segundo o gráfico 5.47 o número de minimizações sem restrições apresentadas pelas combinações com a regra do trapézio são menores que os apresentados com as combinações com splines cúbicos. E ainda as combinações com o método de Newmark obtiveram melhores desempenhos que as combinações com Runge-Kutta de quarta e quinta ordem.

Tabela 5.59 - Valores finais da função objetivo para diferentes valores de $\boldsymbol{x}_{0}$.

\begin{tabular}{|l|c|c|c|c|c|c|c|}
\cline { 2 - 8 } \multicolumn{1}{c|}{} & \multicolumn{6}{c|}{$\mathbf{f}(\mathbf{X f})$} \\
\cline { 2 - 8 } \multicolumn{1}{c|}{} & $\mathbf{f ( X o )}$ & Newm/Splin & Newm/Trap & Rk4/Splin & Rk4/Trap & Rk5/Splin & Rk5/Trap \\
\hline CASO 1 & 17143 & 17824 & 17843 & 18651 & 18684 & 18522 & 18625 \\
\hline CASO 2 & 19166 & 17966 & 17992 & 19050 & 19125 & 18597 & 18742 \\
\hline
\end{tabular}

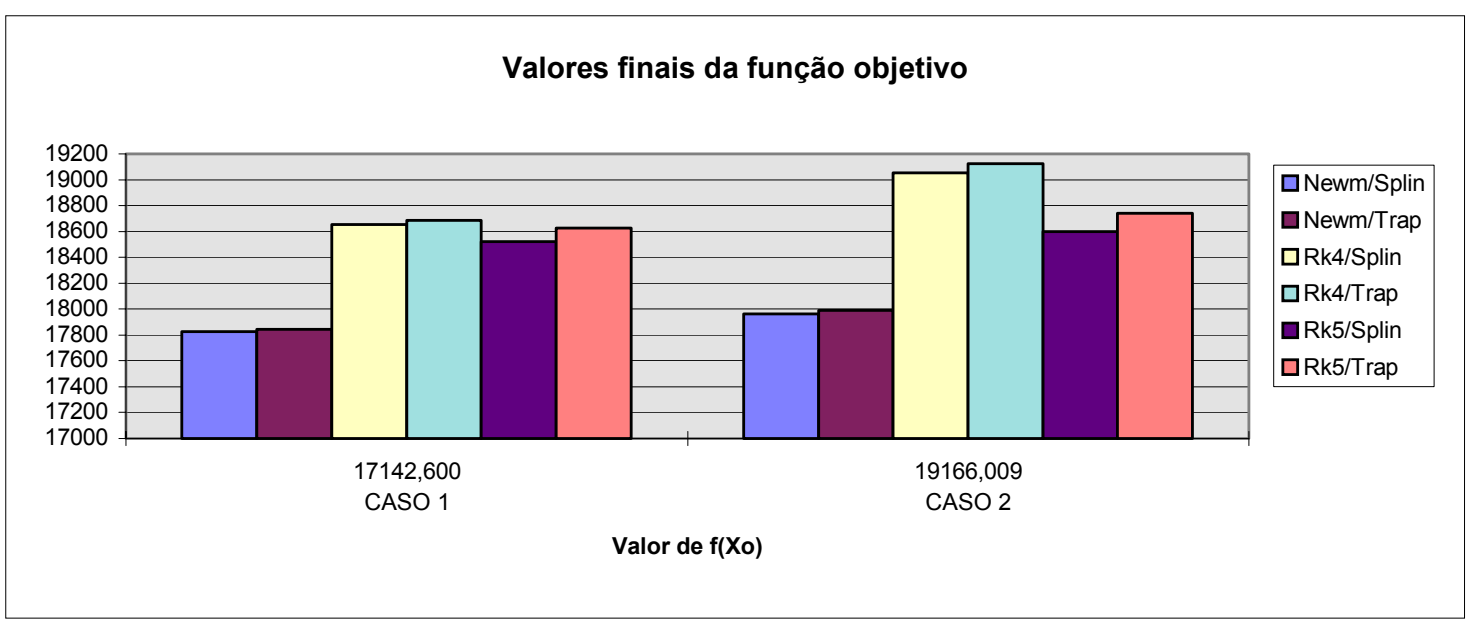

Gráfico 5.48 - Valores finais da função objetivo para diferentes valores de $\boldsymbol{x}_{\mathrm{o}}$ (tabela 5.59).

Novamente se observa que as combinações com o método de Newmark apresentam melhores valores que os dos métodos Runge-Kutta.

Tabela 5.60 - Número de minimizações sem restrições para diferentes valores de $\boldsymbol{x}_{0}$.

\begin{tabular}{|l|c|c|c|c|c|c|}
\cline { 2 - 7 } \multicolumn{1}{c|}{} & \multicolumn{7}{c|}{ K } \\
\cline { 2 - 7 } \multicolumn{1}{c|}{} & Newm/Splin & Newm/Trap & Rk4/Splin & Rk4/Trap & Rk5/Splin & Rk5/Trap \\
\hline CASO 1 & 26 & 24 & 27 & 26 & 26 & 26 \\
\hline CASO 2 & 25 & 23 & 27 & 27 & 26 & 25 \\
\hline
\end{tabular}

A tabela acima mostra que as combinações de métodos com a regra do trapézio apresentam melhore desempenho que as combinações com splines cúbico e quadratura de GaussLegendre. 


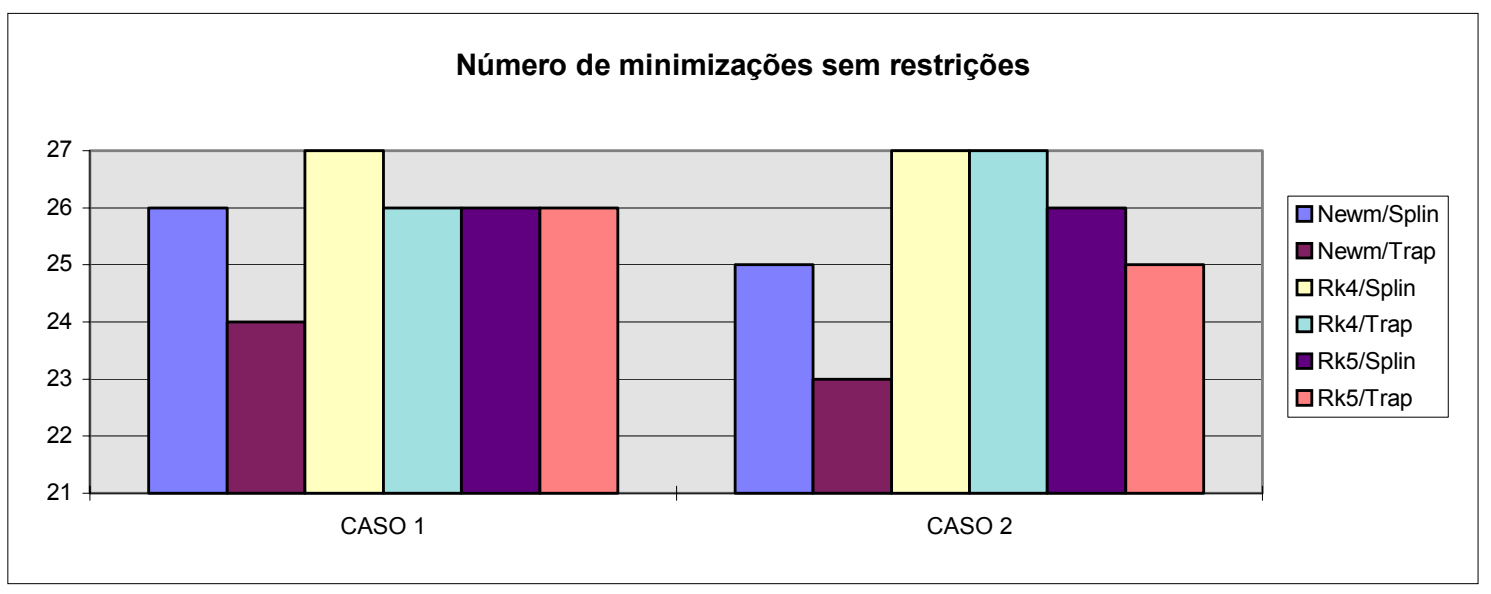

Gráfico 5.49 - Número de minimizações sem restrições para diferentes valores de $\boldsymbol{x}_{\mathrm{o}}$ (Tabela 5.60).

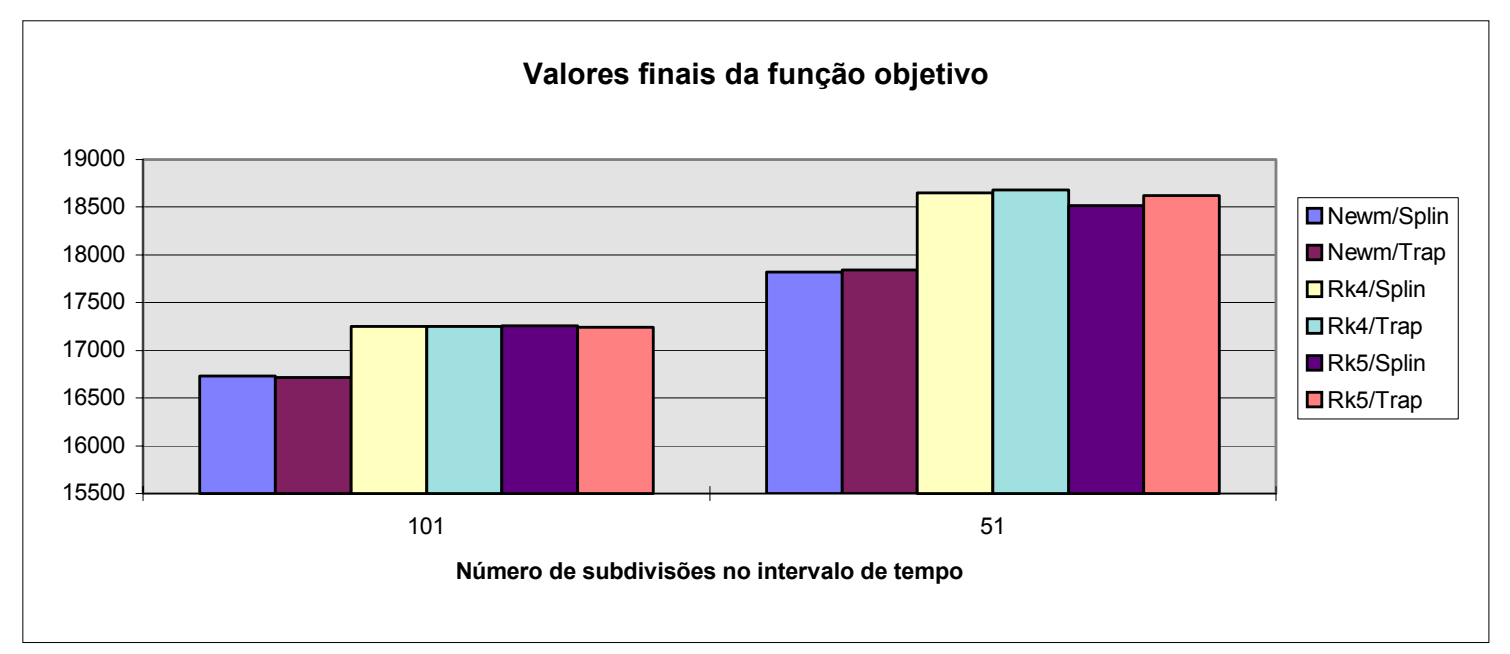

Gráfico 5.50 - Valores finais da função objetivo no caso 1 para diferentes valores de $n$.

Oberva-se neste exemplo que menores valores da função objetivo foram apresentados pela combinação do método de Newmark. Já as combinações de métodos com spline apresentaram menores valores da função objetivo. 


\subsection{EXEMPLO 8 (Arora, Chahande e Paeng [11])}

\section{Shear-building com cinquenta graus de liberdade submetido à explosão}

Este exemplo é semelhante ao exemplo 5.7, porém com 50 graus de liberdade, conforme mostra figura 5.10a. O edifício é solicitado por uma explosão próxima aos cinco últimos pisos. O objetivo é minimizar o peso das colunas. As variáveis de projeto são as rigidezes de cada andar divididas pelo fator de escala 570000. Restrições são impostas às variáveis de projeto, deslocamentos e tensões segundo normas do AISC.

Os pesos dos pisos e paredes estão indicados na figura. Adimite-se que as propriedades são uniformes ao longo do prédio. Todas as colunas do andar são supostas semelhantes (mesma, área, momento de inércia, ...). O pé direito $10 \mathrm{ft}$, e a distância entre as colunas mede $20 \mathrm{ft}$. A massa concentrada em cada grau de liberdade é igual à soma das massas dos pisos e paredes, sendo um total de $816.8 \mathrm{lbs}^{2} / \mathrm{in}$. Utiliza-se o modelo elástico linear representado pelo sistema de massas da figura $5.10 \mathrm{~b}$. A rigidez de um andar é a soma das rigidezes de todos os pilares do andar. A constante elástica de uma coluna do $i$-ésimo andar é

$$
k_{i}=12 E I_{i} / h_{i}{ }^{3},
$$

onde $E$ é o módulo de elasticidade, $I_{i}$ o momento de inércia e $h_{i}$ o comprimento do pilar.

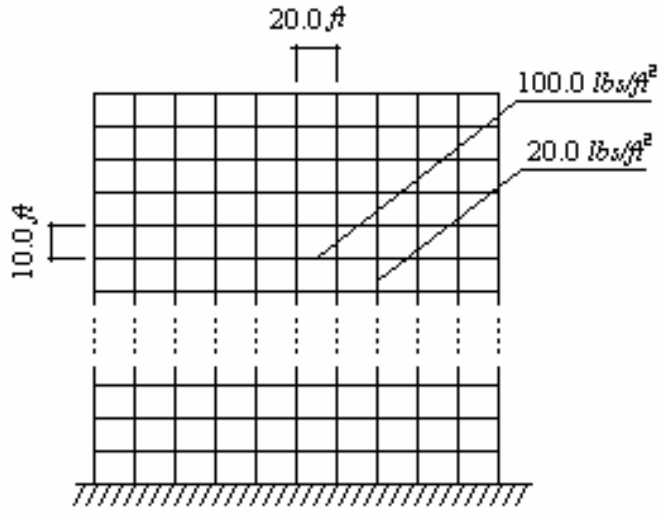

(a)

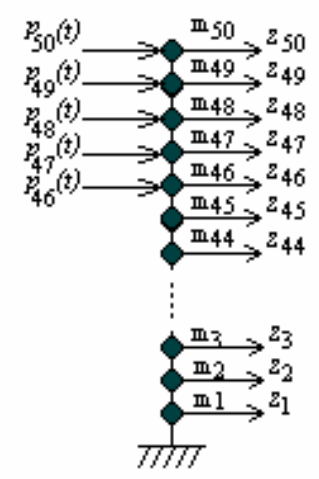

(b)

Figura 5.10 - Shear-building com cinquenta graus de liberdade 
A equação do movimento para o modelo acima é dada de forma explícita:

$$
\left[\begin{array}{ccccc}
m_{1} & 0 & \cdots & 0 & 0 \\
0 & m_{2} & \cdots & 0 & 0 \\
\vdots & \vdots & \ddots & \vdots & \vdots \\
0 & 0 & \cdots & m_{49} & 0 \\
0 & 0 & \cdots & 0 & m_{50}
\end{array}\right]\left[\begin{array}{c}
z_{1}(t) \\
\ddot{z} \\
z_{2}(t) \\
\vdots \\
z_{49}(t) \\
z_{50}(t)
\end{array}\right]+\left[\begin{array}{ccccc}
K_{1}+K_{2} & -K_{2} & \cdots & 0 & 0 \\
-K_{2} & K_{2}+K_{3} & \cdots & 0 & 0 \\
\vdots & \vdots & \ddots & \vdots & \vdots \\
0 & 0 & \cdots & K_{49}+K_{50} & -K_{50} \\
0 & 0 & \cdots & -K_{50} & K_{50}
\end{array}\right]\left[\begin{array}{c}
z_{1}(t) \\
z_{2}(t) \\
\vdots \\
z_{49}(t) \\
z_{50}(t)
\end{array}\right]=\left[\begin{array}{c}
p_{1}(t) \\
p_{2}(t) \\
\vdots \\
p_{49}(t) \\
p_{50}(t)
\end{array}\right]
$$

sendo que $t \in[0,5.0] s$, e as condições iniciais $\boldsymbol{z}(0)=\mathbf{0}$ e $\boldsymbol{z}(0)=\mathbf{0}$. Resumidamente podese escrever: $\boldsymbol{M} \ddot{\boldsymbol{z}}(t)+\boldsymbol{K} \boldsymbol{z}(t)=\boldsymbol{p}(t), t \in[0,5.0]$ s com condições iniciais nulas.

O vetor força generalizada é definido por

$$
\begin{aligned}
& p_{i}(t)=0.0 l b, \quad 0 \leq t \leq 5.0 s, \quad i=1, \ldots, 45 \\
& p_{i}(t)=\left\{\begin{array}{lr}
20000\left(1.0-t / t_{d}\right) l b s, & 0 \leq t \leq t_{d} \\
0 l b, & t_{d} \leq t \leq 5.0 s
\end{array}\right\}, \quad i=46, \ldots, 50
\end{aligned}
$$

sendo que $t_{d}$, o tempo de detonação, é igual à $0.1 \mathrm{~s}$.

Dois tipo de restrições dinâmicas são consideradas, sobre deslocamento e tensões. $\mathrm{O}$ máximo deslocamento relativo permitido entre pisos adjacentes é 0.33333 in e a máxima tensão nos pilares 14400 psi. Os valores máximo e mínimo para as variáveis de projeto são respectivamente 100.0 e $0.0001 \mathrm{lb} / \mathrm{in}$. A densidade do aço é dada por $0.28 \mathrm{lb} / \mathrm{in}^{3}$.

A área da seção transversal e módulo de resistência à flexão dos pilares podem ser obtido por

$$
\begin{aligned}
& A_{i}=0.465 I_{i}^{1 / 2} \mathrm{e} \\
& S_{i}=0.544 I_{i}^{3 / 4} .
\end{aligned}
$$

A rigidez do $i$-ésimo pilar é (11 colunas)

$$
K_{i}=11 k_{i}
$$


Considerando $E=3010^{6}$ psi e $h_{i}=10 \times 12=120$ in obtem-se

$$
K_{i}=2291.6667 I_{i} .
$$

As tensões atuante nos pilares são

$$
\sigma_{1}=K_{1} z_{1} / 11 \text { e } \sigma_{i}=K_{i}\left(z_{i}-z_{i-1}\right) / 11, i=2, \ldots, 50 .
$$

O problema de programação matemática pode ser resumido por

minimizar

$$
\begin{aligned}
f(\boldsymbol{x}) & =11 \rho \sum_{i=1}^{50} A_{i} h_{i} \quad l b \\
& =11(0.28)(0.465) 120 \sum_{i=1}^{50}\left(k_{i} / 2291.6667\right)^{0.5} \\
& =11(0.28)(0.465) 120 \sum_{i=1}^{50}\left(570000 x_{i} / 2291.6667\right)^{0.5} \\
& =2710.483 \sum_{i=1}^{50} x_{i}^{0.5}
\end{aligned}
$$

Sujeito a:

1. restrições sobre deslocamento (desigualdades)

$$
\begin{aligned}
& g_{1}=\left|z_{1}(t)\right|-0.33333, \quad t \in[0,0.3] ; \\
& g_{i}=\left|z_{i}(t)-z_{i-1}(t)\right|-0.33333, \quad t \in[0,0.3] ; \quad i=2, \ldots, 50 .
\end{aligned}
$$

2. restrições sobre tensões (desigualdades)

$$
\begin{aligned}
& g_{11}=570000 x_{1}\left|z_{1}(t)\right| /\left[11 A_{i}(\boldsymbol{x})\right]-14400, \quad t \in[0,0.3] ; \\
& g_{(i+10)}=570000 x_{i}\left|z_{i}(t)-z_{i-1}(t)\right| /\left[11 A_{i}(\boldsymbol{x})\right]-14400, \quad t \in[0,0.3] ; \quad i=2, \ldots, 50 .
\end{aligned}
$$

3. valores máximos e mínimos para as variáveis de projeto

$$
0.0001 \leq x_{i} \leq 100.0, \quad i=1, \ldots, 50 \text {. }
$$

O exemplo foi resolvido para dois valores iniciais de $\boldsymbol{x}$, o caso 1 e o caso 2 . Um número de 50 subdivisões nas tabelas 5.61 à 5.63 e 40 subdivisões nas tabelas 5.64 à 5.66 foram adotadas na discretização do intervalo de tempo $[0,5.0] \mathrm{s}$. 
Tabela 5.61a - Valores finais das variáveis de projeto para diferentes valores de $\boldsymbol{x}_{0}$.

\begin{tabular}{|c|c|c|c|c|c|c|c|c|}
\hline & & \multicolumn{6}{|c|}{$\mathbf{X T}$} \\
\hline & & Xo & Newm/Splin & \begin{tabular}{|l|l} 
Newm/Trap \\
\end{tabular} & Rk4/Splin & Rk4/Trap & Rk5/Splin & Rk5/Trap \\
\hline \multirow{50}{*}{ CASO 1} & $\mathrm{X1}$ & 1,000 & 0,384 & 0,418 & $*$ & $*$ & 0,940 & $*$ \\
\hline & $\mathrm{X2}$ & 1,000 & 2,210 & 2,176 & * & * & 0,848 & \\
\hline & $\mathrm{X3}$ & 1,000 & 2,018 & 2,002 & * & * & 0,795 & * \\
\hline & $\mathrm{X} 4$ & 1,000 & 1,818 & 1,819 & * & * & 0,778 & * \\
\hline & $X 5$ & 1,000 & 1,594 & 1,612 & * & * & 0,775 & * \\
\hline & $\mathrm{X6}$ & 1,000 & 1,236 & 1,274 & * & * & 0,773 & * \\
\hline & $\mathrm{X7}$ & 1,000 & 0,209 & 0,560 & * & * & 0,770 & * \\
\hline & $\mathrm{X8}$ & 1,000 & 0,422 & 0,219 & * & * & 0,772 & * \\
\hline & $X 9$ & 1,000 & 0,943 & 0,864 & * & * & 0,769 & * \\
\hline & $\mathrm{X} 10$ & 1,000 & 1,222 & 1,165 & * & * & 0,765 & * \\
\hline & $\mathrm{X} 11$ & 1,000 & 1,405 & 1,362 & * & * & 0,764 & * \\
\hline & $\mathrm{X} 12$ & 1,000 & 1,477 & 1,442 & * & * & 0,758 & * \\
\hline & X13 & 1,000 & 1,480 & 1,444 & * & * & 0,760 & * \\
\hline & X14 & 1,000 & 1,448 & 1,410 & * & * & 0,758 & * \\
\hline & X15 & 1,000 & 1,387 & 1,361 & * & * & 0,775 & * \\
\hline & $\mathrm{X} 16$ & 1,000 & 1,292 & 1,291 & * & * & 0,775 & * \\
\hline & X17 & 1,000 & 1,175 & 1,191 & * & * & 0,789 & * \\
\hline & X18 & 1,000 & 1,056 & 1,070 & * & * & 0,804 & * \\
\hline & $\mathrm{X} 19$ & 1,000 & 1,010 & 1,007 & * & * & 0,804 & * \\
\hline & $\mathrm{X20}$ & 1,000 & 1,068 & 1,059 & * & * & 0,809 & * \\
\hline & X21 & 1,000 & 1,165 & 1,153 & * & * & 0,821 & * \\
\hline & $\mathrm{X} 22$ & 1,000 & 1,224 & 1,215 & * & * & 0,817 & * \\
\hline & $\mathrm{X23}$ & 1,000 & 1,254 & 1,245 & * & * & 0,819 & * \\
\hline & $\mathrm{X24}$ & 1,000 & 1,241 & 1,224 & * & * & 0,842 & * \\
\hline & $\mathrm{X25}$ & 1,000 & 1,187 & 1,174 & * & * & 0,807 & * \\
\hline & $\mathrm{X26}$ & 1,000 & 1,147 & 1,138 & * & * & 0,799 & * \\
\hline & $\mathbf{X 2 7}$ & 1,000 & 1,160 & 1,142 & * & * & 0,833 & * \\
\hline & $\mathrm{X28}$ & 1,000 & 1,190 & 1,155 & * & * & 0,857 & * \\
\hline & $\mathrm{X29}$ & 1,000 & 1,218 & 1,178 & * & * & 0,837 & * \\
\hline & X30 & 1,000 & 1,257 & 1,212 & * & * & 0,749 & * \\
\hline & X31 & 1,000 & 1,301 & 1,262 & * & * & 0,804 & * \\
\hline & X32 & 1,000 & 1,262 & 1,235 & * & * & 0,838 & * \\
\hline & X33 & 1,000 & 1,182 & 1,166 & * & * & 0,790 & * \\
\hline & X34 & 1,000 & 1,000 & 0,976 & * & * & 0,865 & * \\
\hline & X35 & 1,000 & 0,727 & 0,696 & * & * & 0,785 & * \\
\hline & X36 & 1,000 & 0,349 & 0,331 & * & * & 0,790 & * \\
\hline & X37 & 1,000 & 0,388 & 0,376 & * & * & 0,786 & * \\
\hline & X38 & 1,000 & 0,901 & 0,900 & * & * & 0,789 & * \\
\hline & X39 & 1,000 & 1,190 & 1,195 & * & * & 0,843 & * \\
\hline & X40 & 1,000 & 1,420 & 1,418 & * & * & 0,834 & * \\
\hline & X41 & 1,000 & 1,575 & 1,557 & * & * & 0,691 & * \\
\hline & X42 & 1,000 & 1,685 & 1,664 & * & * & 0,903 & * \\
\hline & X43 & 1,000 & 1,723 & 1,701 & * & * & 0,876 & * \\
\hline & X44 & 1,000 & 1,732 & 1,716 & * & * & 0,812 & * \\
\hline & X45 & 1,000 & 1,606 & 1,598 & * & $\star$ & 0,778 & 7 \\
\hline & X46 & 1,000 & 0,672 & 0,704 & * & * & 0,750 & * \\
\hline & X47 & 1,000 & 1,162 & 1,160 & * & * & 0,649 & * \\
\hline & X48 & 1,000 & 0,999 & 0,986 & * & * & 0,788 & * \\
\hline & X49 & 1,000 & 0,686 & 0,620 & * & * & 0,018 & * \\
\hline & $\begin{array}{l}50 \\
\end{array}$ & 1,000 & 0,886 & 0,858 & * & 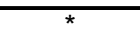 & 0,793 & 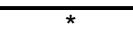 \\
\hline
\end{tabular}


Tabela 5.61b - Valores finais das variáveis de projeto para diferentes valores de $\boldsymbol{x}_{0}$.

\begin{tabular}{|c|c|c|c|c|c|c|c|c|}
\hline & \multirow[b]{2}{*}{ Xo } & \multicolumn{6}{|c|}{$\mathbf{X f}$} \\
\hline & & & Newm/Splin & Newm/Trap & Rk4/Splin & Rk4/Trap & Rk5/Splin & Rk5/Trap \\
\hline \multirow{50}{*}{ CASO 2} & $\mathrm{X1}$ & 1,100 & 0,364 & 0,388 & ${ }^{*}$ & $*$ & & \\
\hline & $\mathrm{X} 2$ & 1,100 & 2,072 & 2,055 & * & * & * & * \\
\hline & $x 3$ & 1,100 & 1,903 & 1,901 & * & * & * & * \\
\hline & $\mathrm{X4}$ & 1,100 & 1,745 & 1,773 & * & * & * & * \\
\hline & $\times 5$ & 1,100 & 1,612 & 1,639 & * & * & * & * \\
\hline & $\mathrm{X6}$ & 1,100 & 1,392 & 1,377 & * & * & * & * \\
\hline & $\mathrm{X7}$ & 1,100 & 1,002 & 0,904 & * & * & * & * \\
\hline & $X 8$ & 1,100 & 0,202 & 0,179 & * & * & * & * \\
\hline & $\mathrm{X9}$ & 1,100 & 0,625 & 0,759 & * & * & * & * \\
\hline & $\mathrm{X10}$ & 1,100 & 1,002 & 1,087 & * & * & * & * \\
\hline & $\mathrm{X11}$ & 1,100 & 1,192 & 1,245 & * & * & * & * \\
\hline & $\mathrm{X12}$ & 1,100 & 1,300 & 1,310 & * & * & * & * \\
\hline & $\mathrm{X13}$ & 1,100 & 1,388 & 1,343 & * & * & * & * \\
\hline & $\mathrm{X14}$ & 1,100 & 1,450 & 1,356 & * & * & * & * \\
\hline & $\mathrm{X15}$ & 1,100 & 1,506 & 1,373 & * & * & * & * \\
\hline & $\mathrm{X} 16$ & 1,100 & 1,549 & 1,395 & * & * & * & * \\
\hline & $\mathrm{X17}$ & 1,100 & 1,554 & 1,390 & * & * & * & * \\
\hline & $\mathrm{X} 18$ & 1,100 & 1,531 & 1,375 & * & * & * & * \\
\hline & $\mathrm{X} 19$ & 1,100 & 1,463 & 1,326 & * & * & * & * \\
\hline & $\mathbf{X 2 0}$ & 1,100 & 1,396 & 1,298 & * & * & * & * \\
\hline & $\mathrm{X21}$ & 1,100 & 1,350 & 1,275 & * & * & * & * \\
\hline & $\mathrm{X22}$ & 1,100 & 1,312 & 1,239 & * & * & * & * \\
\hline & $\mathrm{X23}$ & 1,100 & 1,303 & 1,212 & * & * & * & * \\
\hline & $\mathrm{X24}$ & 1,100 & 1,346 & 1,221 & * & * & * & * \\
\hline & $\mathrm{X25}$ & 1,100 & 1,410 & 1,260 & * & * & * & * \\
\hline & $\mathrm{X26}$ & 1,100 & 1,468 & 1,313 & * & * & * & * \\
\hline & $\mathbf{X 2 7}$ & 1,100 & 1,516 & 1,360 & * & * & * & * \\
\hline & $\mathrm{X28}$ & 1,100 & 1,524 & 1,394 & * & * & * & * \\
\hline & $\mathrm{X29}$ & 1,100 & 1,477 & 1,382 & * & * & * & * \\
\hline & $\mathbf{X 3 0}$ & 1,100 & 1,378 & 1,337 & * & * & * & * \\
\hline & $\mathrm{X} 31$ & 1,100 & 1,248 & 1,285 & * & * & * & * \\
\hline & $\mathrm{X32}$ & 1,100 & 1,121 & 1,223 & * & * & * & * \\
\hline & $\mathrm{X33}$ & 1,100 & 0,974 & 1,118 & * & * & * & * \\
\hline & X34 & 1,100 & 0,904 & 0,991 & * & * & * & * \\
\hline & X35 & 1,100 & 0,960 & 0,879 & * & * & * & * \\
\hline & X36 & 1,100 & 1,047 & 0,757 & * & * & * & * \\
\hline & X37 & 1,100 & 1,195 & 0,811 & * & * & * & * \\
\hline & $\mathrm{X38}$ & 1,100 & 1,383 & 1,079 & * & * & * & * \\
\hline & X39 & 1,100 & 1,542 & 1,313 & * & * & * & * \\
\hline & $\mathbf{X 4 0}$ & 1,100 & 1,651 & 1,501 & * & * & * & * \\
\hline & $\mathrm{X41}$ & 1,100 & 1,726 & 1,641 & * & 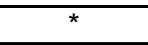 & * & 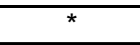 \\
\hline & $\mathrm{X42}$ & 1,100 & 1,749 & 1,711 & * & * & * & * \\
\hline & $\mathrm{X43}$ & 1,100 & 1,764 & 1,742 & * & * & * & * \\
\hline & X44 & 1,100 & 1,783 & 1,760 & * & * & * & * \\
\hline & X45 & 1,100 & 1,706 & 1,684 & * & * & * & * \\
\hline & X46 & 1,100 & 0,534 & 0,535 & * & * & * & * \\
\hline & X47 & 1,100 & 1,092 & 1,053 & * & * & * & * \\
\hline & X48 & 1,100 & 0,783 & 0,676 & * & * & * & * \\
\hline & $\mathbf{X 4 9}$ & 1,100 & 0,482 & 0,585 & * & * & * & * \\
\hline & $\mathrm{X50}$ & 1,100 & 0,909 & 0,938 & - & 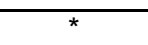 & ${ }^{*}$ & 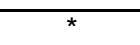 \\
\hline
\end{tabular}


Tabela 5.62 - Valores finais da função objetivo para diferentes valores de $\boldsymbol{x}_{0}$.

\begin{tabular}{|c|c|c|c|c|c|c|c|}
\cline { 2 - 8 } \multicolumn{1}{c|}{} & $\mathbf{f ( X o )}$ & Newm/Splin & Newm/Trap & Rk4/Splin & Rk4/Trap & Rk5/Splin & Rk5/Trap \\
\cline { 2 - 8 } \multicolumn{1}{c|}{} & 135524 & 144890 & 144202 & ${ }^{*}$ & ${ }^{*}$ & 118991 & ${ }^{*}$ \\
\hline CASO 1 & 142139 & 152019 & 148369 & ${ }^{*}$ & ${ }^{*}$ & ${ }^{*}$ & ${ }^{*}$ \\
\hline CASO 2 & 1429 &
\end{tabular}

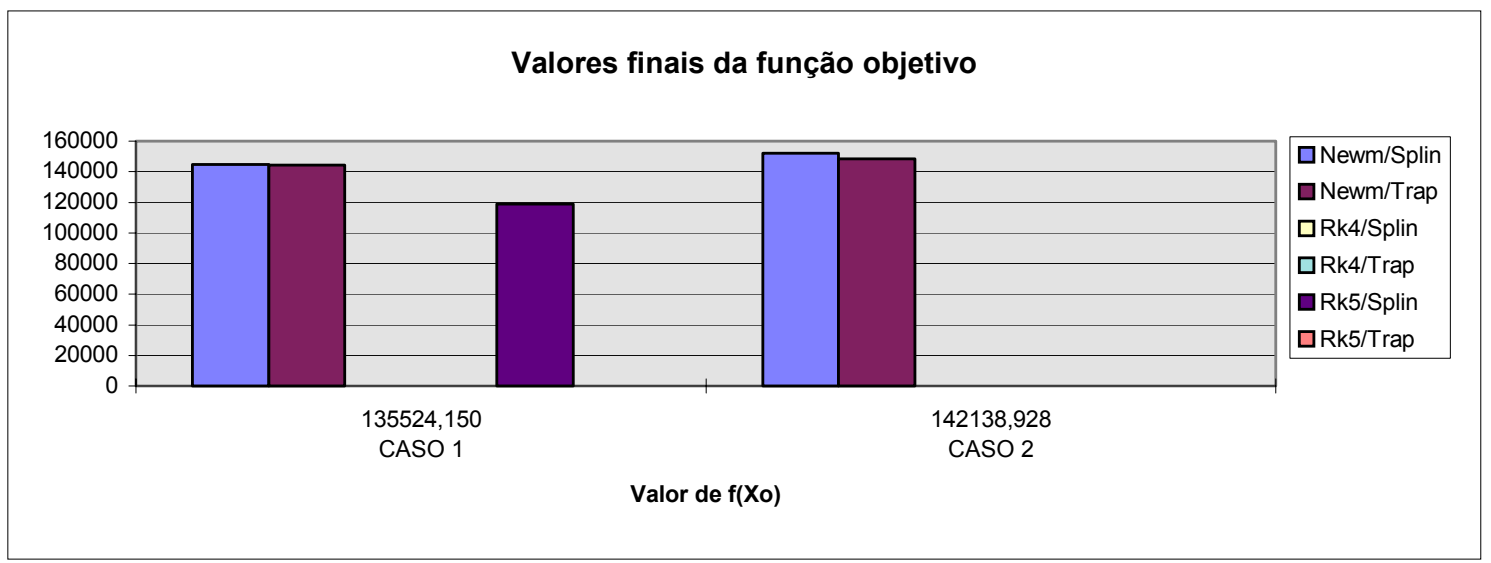

Gráfico 5.51 - Valores finais da função objetivo para diferentes valores de $\boldsymbol{x}_{\mathrm{o}}$ (tabela 5.62).

Tabela 5.63 - Número de minimizações sem restrições para diferentes valores de $\boldsymbol{x}_{0}$.

\begin{tabular}{|l|c|c|c|c|c|c|}
\cline { 2 - 7 } \multicolumn{1}{c|}{} & \multicolumn{7}{c|}{ K } \\
\cline { 2 - 7 } \multicolumn{1}{c|}{} & Newm/Splin & Newm/Trap & Rk4/Splin & Rk4/Trap & Rk5/Splin & Rk5/Trap \\
\hline CASO 1 & 34 & 38 & ${ }^{*}$ & ${ }^{*}$ & 43 & ${ }^{*}$ \\
\hline CASO 2 & 35 & 38 & $*$ & $*$ & ${ }^{*}$ & ${ }^{*}$ \\
\hline
\end{tabular}

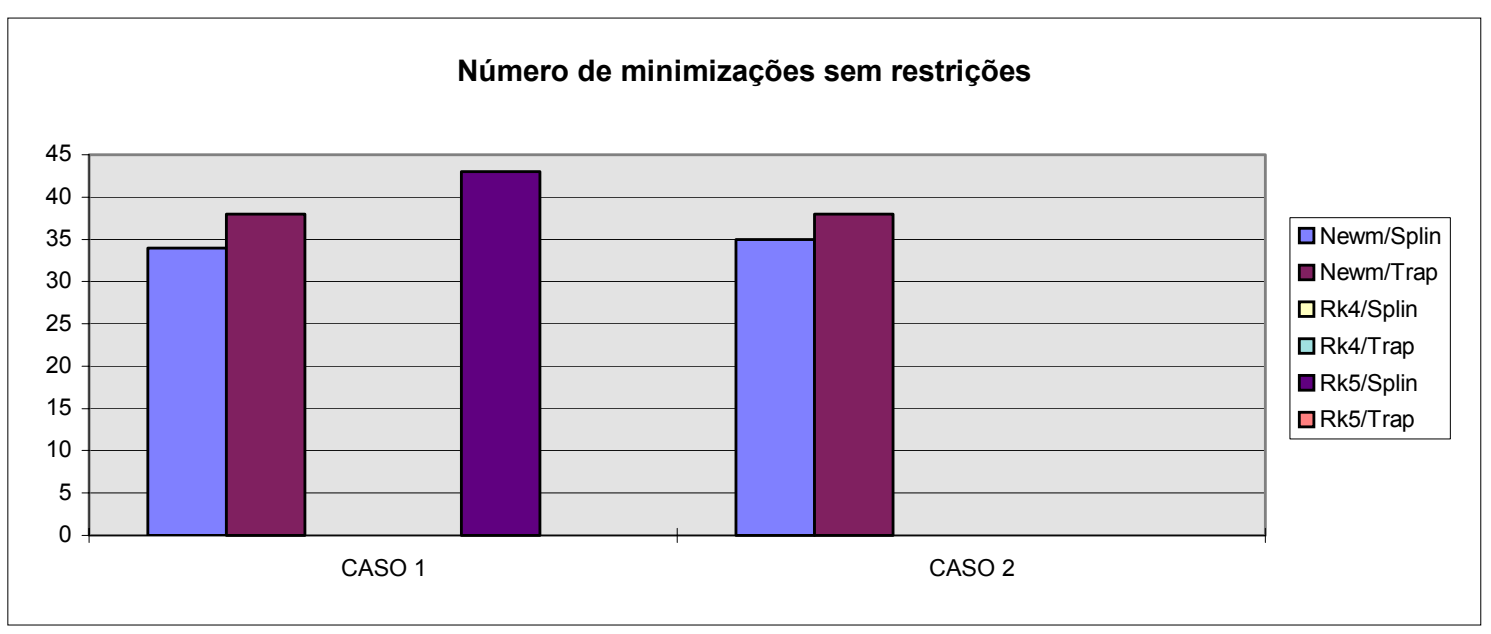

Gráfico 5.52 - Número de minimizações sem restrições para diferentes valores de $\boldsymbol{x}_{\mathrm{0}}$ (Tabela 5.63). 
Tabela 5.64a - Valores finais das variáveis de projeto para diferentes valores de $\boldsymbol{x}_{0}$.

\begin{tabular}{|c|c|c|c|c|c|c|c|c|}
\hline & \\
\hline & & Xo & Newm/Splin & Newm/Trap & Rk4/Splin & Rk4/Trap & Rk5/Splin & Rk5/Trap \\
\hline \multirow{50}{*}{ CASO 1} & $\mathrm{X1}$ & 1,000 & 0,245 & 0,232 & & & & \\
\hline & $\mathrm{X} 2$ & 1,000 & 2,000 & 1,621 & * & * & * & * \\
\hline & $\mathrm{X3}$ & 1,000 & 1,709 & 1,571 & * & * & * & * \\
\hline & $\mathrm{X4}$ & 1,000 & 1,289 & 1,497 & * & * & * & * \\
\hline & $\mathrm{X5}$ & 1,000 & 0,624 & 1,398 & * & * & * & * \\
\hline & $\mathrm{X6}$ & 1,000 & 0,441 & 1,233 & * & * & * & * \\
\hline & $\mathrm{X7}$ & 1,000 & 1,048 & 0,973 & * & * & * & * \\
\hline & $X 8$ & 1,000 & 1,310 & 0,596 & * & * & * & * \\
\hline & $X 9$ & 1,000 & 1,407 & 0,143 & * & * & * & * \\
\hline & $\mathbf{X 1 0}$ & 1,000 & 1,346 & 0,606 & * & * & * & * \\
\hline & X11 & 1,000 & 1,315 & 0,876 & * & * & * & * \\
\hline & $\mathrm{X} 12$ & 1,000 & 1,054 & 1,044 & * & * & * & * \\
\hline & $\mathrm{X} 13$ & 1,000 & 0,739 & 1,145 & * & * & * & * \\
\hline & X14 & 1,000 & 0,612 & 1,223 & * & * & * & * \\
\hline & $\mathrm{X} 15$ & 1,000 & 0,868 & 1,276 & * & * & * & * \\
\hline & $\mathrm{X} 16$ & 1,000 & 1,137 & 1,284 & * & * & * & * \\
\hline & X17 & 1,000 & 1,335 & 1,268 & * & * & * & * \\
\hline & X18 & 1,000 & 1,475 & 1,254 & * & * & * & * \\
\hline & $\mathrm{X} 19$ & 1,000 & 1,564 & 1,248 & * & * & * & * \\
\hline & $\mathrm{X20}$ & 1,000 & 1,605 & 1,239 & * & * & * & * \\
\hline & $\mathrm{X21}$ & 1,000 & 1,601 & 1,222 & * & * & * & * \\
\hline & $\mathrm{X22}$ & 1,000 & 1,555 & 1,232 & * & * & * & * \\
\hline & $\mathrm{X23}$ & 1,000 & 1,464 & 1,249 & * & * & * & * \\
\hline & $\mathrm{X24}$ & 1,000 & 1,326 & 1,249 & * & * & * & * \\
\hline & $\mathrm{X25}$ & 1,000 & 1,145 & 1,238 & * & * & * & * \\
\hline & $\mathrm{X26}$ & 1,000 & 0,923 & 1,247 & * & * & * & * \\
\hline & $\mathbf{X 2 7}$ & 1,000 & 0,674 & 1,243 & * & * & * & * \\
\hline & $\mathrm{X28}$ & 1,000 & 0,414 & 1,182 & * & * & * & * \\
\hline & $\mathbf{X 2 9}$ & 1,000 & 0,356 & 1,094 & * & * & * & * \\
\hline & X30 & 1,000 & 0,630 & 0,998 & * & * & * & * \\
\hline & X31 & 1,000 & 0,908 & 0,912 & * & * & * & * \\
\hline & X32 & 1,000 & 1,135 & 0,860 & * & * & * & * \\
\hline & X33 & 1,000 & 1,299 & 0,841 & * & * & * & * \\
\hline & X34 & 1,000 & 1,400 & 0,949 & * & * & * & * \\
\hline & $\begin{array}{r}35 \\
\end{array}$ & 1,000 & 1,434 & 1,051 & * & * & * & * \\
\hline & X36 & 1,000 & 1,405 & 1,085 & * & * & * & * \\
\hline & X37 & 1,000 & 1,313 & 1,161 & * & * & * & * \\
\hline & X38 & 1,000 & 1,160 & 1,241 & * & * & * & * \\
\hline & X39 & 1,000 & 0,986 & 1,327 & * & * & * & * \\
\hline & $\mathbf{X 4 0}$ & 1,000 & 0,937 & 1,401 & * & * & * & * \\
\hline & X41 & 1,000 & 1,143 & 1,464 & * & * & * & * \\
\hline & X42 & 1,000 & 1,408 & 1,490 & * & * & * & * \\
\hline & X43 & 1,000 & 1,595 & 1,499 & * & * & * & * \\
\hline & X44 & 1,000 & 1,637 & 1,484 & * & * & * & * \\
\hline & X45 & 1,000 & 1,459 & 1,372 & * & * & * & * \\
\hline & X46 & 1,000 & 0,601 & 0,330 & * & * & * & * \\
\hline & X47 & 1,000 & 1,123 & 0,822 & * & * & * & * \\
\hline & X48 & 1,000 & 1,172 & 0,424 & * & * & * & * \\
\hline & X49 & 1,000 & 1,103 & 0,318 & * & * & * & * \\
\hline & X50 & 1,000 & 1,031 & 0,827 & 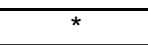 & * & * & * \\
\hline
\end{tabular}


Tabela 5.64b - Valores finais das variáveis de projeto para diferentes valores de $\boldsymbol{x}_{0}$.

\begin{tabular}{|c|c|c|c|c|c|c|c|c|}
\hline & & \\
\hline & & Xo & Newm/Splin & Newm/Trap & Rk4/Splin & Rk4/Trap & Rk5/Splin & Rk5/Trap \\
\hline \multirow{50}{*}{ CASO 2} & $\overline{X 1}$ & 1,100 & 0,271 & $\begin{array}{l}0,229 \\
\end{array}$ & * & * & & * \\
\hline & $\mathrm{X} 2$ & 1,100 & 1,775 & 1,605 & * & * & * & * \\
\hline & $\mathrm{X3}$ & 1,100 & 1,727 & 1,591 & * & * & * & * \\
\hline & $\mathrm{X} 4$ & 1,100 & 1,647 & 1,560 & * & * & * & * \\
\hline & $\mathrm{X} 5$ & 1,100 & 1,522 & 1,493 & * & * & * & * \\
\hline & $\mathrm{X6}$ & 1,100 & 1,328 & 1,347 & * & * & * & * \\
\hline & $\mathrm{X7}$ & 1,100 & 1,044 & 1,094 & * & * & * & * \\
\hline & $\mathrm{X8}$ & 1,100 & 0,670 & 0,733 & * & * & * & * \\
\hline & $X 9$ & 1,100 & 0,154 & 0,158 & * & * & * & * \\
\hline & $\mathbf{X 1 0}$ & 1,100 & 0,538 & 0,374 & * & * & * & * \\
\hline & $\mathrm{X} 11$ & 1,100 & 0,776 & 0,657 & * & * & * & * \\
\hline & $\mathrm{X} 12$ & 1,100 & 0,949 & 0,845 & * & * & * & * \\
\hline & $\mathrm{X13}$ & 1,100 & 1,114 & 1,025 & * & * & * & * \\
\hline & $\mathrm{X14}$ & 1,100 & 1,275 & 1,202 & * & * & * & * \\
\hline & $\mathbf{X 1 5}$ & 1,100 & 1,405 & 1,343 & * & * & * & * \\
\hline & $\mathrm{X} 16$ & 1,100 & 1,480 & 1,427 & * & * & * & * \\
\hline & $\mathbf{X 1 7}$ & 1,100 & 1,512 & 1,467 & * & * & * & * \\
\hline & $\mathrm{X} 18$ & 1,100 & 1,504 & 1,469 & * & * & * & * \\
\hline & $\mathrm{X19}$ & 1,100 & 1,476 & 1,451 & * & * & * & * \\
\hline & $\mathrm{X20}$ & 1,100 & 1,425 & 1,414 & * & * & * & * \\
\hline & $\mathrm{X21}$ & 1,100 & 1,355 & 1,356 & * & * & * & * \\
\hline & $\mathrm{X22}$ & 1,100 & 1,310 & 1,316 & * & * & * & * \\
\hline & $\mathbf{X 2 3}$ & 1,100 & 1,288 & 1,287 & * & * & * & * \\
\hline & $\mathrm{X24}$ & 1,100 & 1,265 & 1,233 & * & * & * & * \\
\hline & $\mathbf{X 2 5}$ & 1,100 & 1,248 & 1,183 & * & * & * & * \\
\hline & $\mathrm{X26}$ & 1,100 & 1,280 & 1,178 & * & * & * & * \\
\hline & $\mathrm{X} 27$ & 1,100 & 1,300 & 1,171 & * & * & * & * \\
\hline & $\mathrm{X28}$ & 1,100 & 1,252 & 1,105 & * & * & * & * \\
\hline & X29 & 1,100 & 1,175 & 1,042 & * & * & * & * \\
\hline & X30 & 1,100 & 1,070 & 0,972 & * & * & * & * \\
\hline & X31 & 1,100 & 0,914 & 0,879 & * & * & * & * \\
\hline & X32 & 1,100 & 0,747 & 0,825 & * & * & * & * \\
\hline & X33 & 1,100 & 0,542 & 0,784 & * & * & * & * \\
\hline & X34 & 1,100 & 0,669 & 0,877 & * & * & * & * \\
\hline & X35 & 1,100 & 0,905 & 1,038 & * & * & * & * \\
\hline & X36 & 1,100 & 1,073 & 1,137 & * & * & * & * \\
\hline & X37 & 1,100 & 1,251 & 1,244 & * & * & * & * \\
\hline & X38 & 1,100 & 1,414 & 1,362 & * & * & * & * \\
\hline & X39 & 1,100 & 1,534 & 1,456 & * & * & * & * \\
\hline & $\mathrm{X} 40$ & 1,100 & 1,586 & 1,495 & * & * & * & * \\
\hline & $\mathrm{X} 41$ & 1,100 & 1,609 & 1,509 & * & * & * & * \\
\hline & $\mathrm{X} 42$ & 1,100 & 1,615 & 1,518 & * & * & * & * \\
\hline & $\mathrm{X} 43$ & 1,100 & 1,572 & 1,473 & * & * & * & * \\
\hline & $\mathrm{X} 44$ & 1,100 & 1,543 & 1,442 & * & * & * & * \\
\hline & X45 & 1,100 & 1,402 & 1,317 & * & * & * & * \\
\hline & $\mathrm{X} 46$ & 1,100 & 0,311 & 0,255 & * & * & * & * \\
\hline & X47 & 1,100 & 0,862 & 0,779 & * & * & * & * \\
\hline & X48 & 1,100 & 0,657 & 0,548 & * & * & * & * \\
\hline & $\mathrm{X} 49$ & 1,100 & 0,530 & 0,584 & * & * & * & * \\
\hline & $\mathbf{X 5 0}$ & 1,100 & 0,924 & 0,946 & * & * & * & * \\
\hline
\end{tabular}


No gráfico 5.51 observa-se que o método do Lagrangeano aumentado convergiu nas combinações com o método de Newmark. Já nas combinações com os métodos RungeKutta, apenas o de quinta ordem com spines cúbicos no caso 1 convergiu. O número de minimizações sem restrições, segundo o gráfico 5.52 é maior na combinação Runge-Kutta de quinta ordem com splines.

Tabela 5.65 - Valores finais da função objetivo para diferentes valores de $\boldsymbol{x}_{\mathrm{0}}$.

\begin{tabular}{|l|c|c|c|c|c|c|c|}
\cline { 2 - 8 } \multicolumn{1}{c|}{} & \multicolumn{6}{c|}{$\mathbf{f}(\mathbf{X f})$} \\
\cline { 2 - 8 } \multicolumn{1}{c|}{} & $\mathbf{f ( X )}$ & Newm/Splin & Newm/Trap & Rk4/Splin & Rk4/Trap & Rk5/Splin & Rk5/Trap \\
\hline CASO 1 & 135524 & 142782 & 138930 & ${ }^{*}$ & ${ }^{*}$ & ${ }^{*}$ & ${ }^{*}$ \\
\hline CASO 2 & 142139 & 142776 & 140348 & ${ }^{*}$ & ${ }^{*}$ & ${ }^{*}$ & ${ }^{*}$ \\
\hline
\end{tabular}

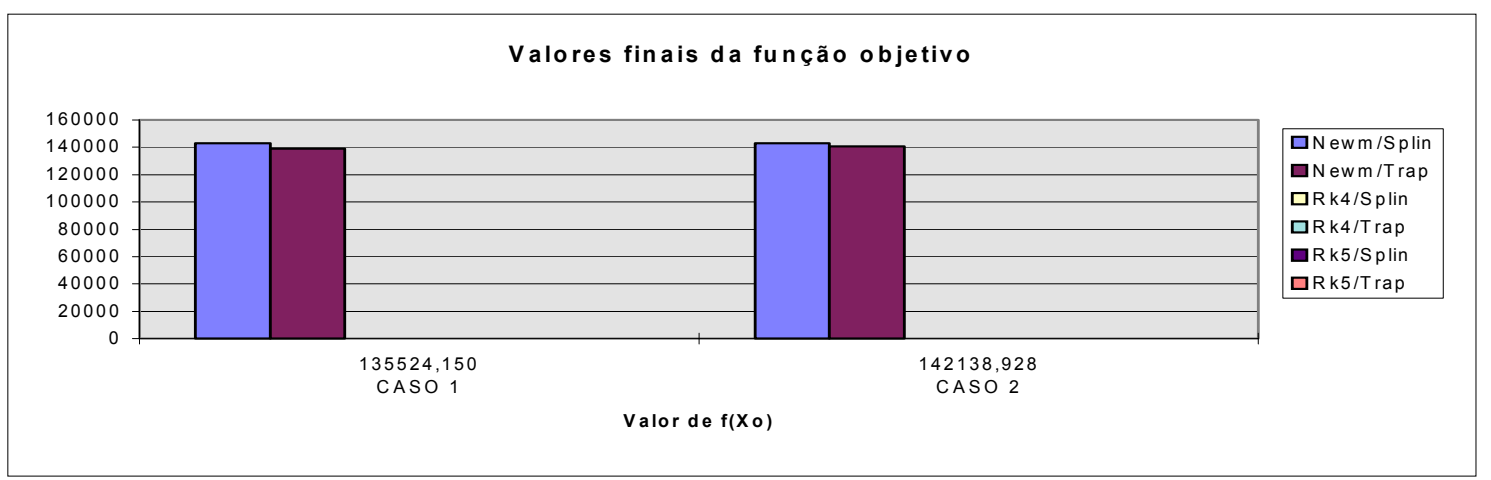

Gráfico 5.53 - Valores finais da função objetivo para diferentes valores de $\boldsymbol{x}_{\mathrm{o}}$ (tabela 5.65).

Tabela 5.66 - Número de minimizações sem restrições para diferentes valores de $\boldsymbol{x}_{0}$.

\begin{tabular}{|l|c|c|c|c|c|c|}
\cline { 2 - 7 } \multicolumn{1}{c|}{} & \multicolumn{7}{c|}{ K } \\
\cline { 2 - 7 } \multicolumn{1}{c|}{} & Newm/Splin & Newm/Trap & Rk4/Splin & Rk4/Trap & Rk5/Splin & Rk5/Trap \\
\hline CASO 1 & 38 & 41 & ${ }^{*}$ & ${ }^{*}$ & ${ }^{*}$ & ${ }^{*}$ \\
\hline CASO 2 & 42 & 46 & ${ }^{*}$ & ${ }^{*}$ & ${ }^{*}$ & \\
\hline
\end{tabular}



Gráfico 5.54 - Número de minimizações sem restrições para diferentes valores de $\boldsymbol{x}_{\mathrm{0}}$ (Tabela 5.66). 
No gráfico 5.53 somente as combinações com Newmark convergiram. Já o gráfico 5.54 mostra um melhor desempenho das combinações com splines cúbicos.

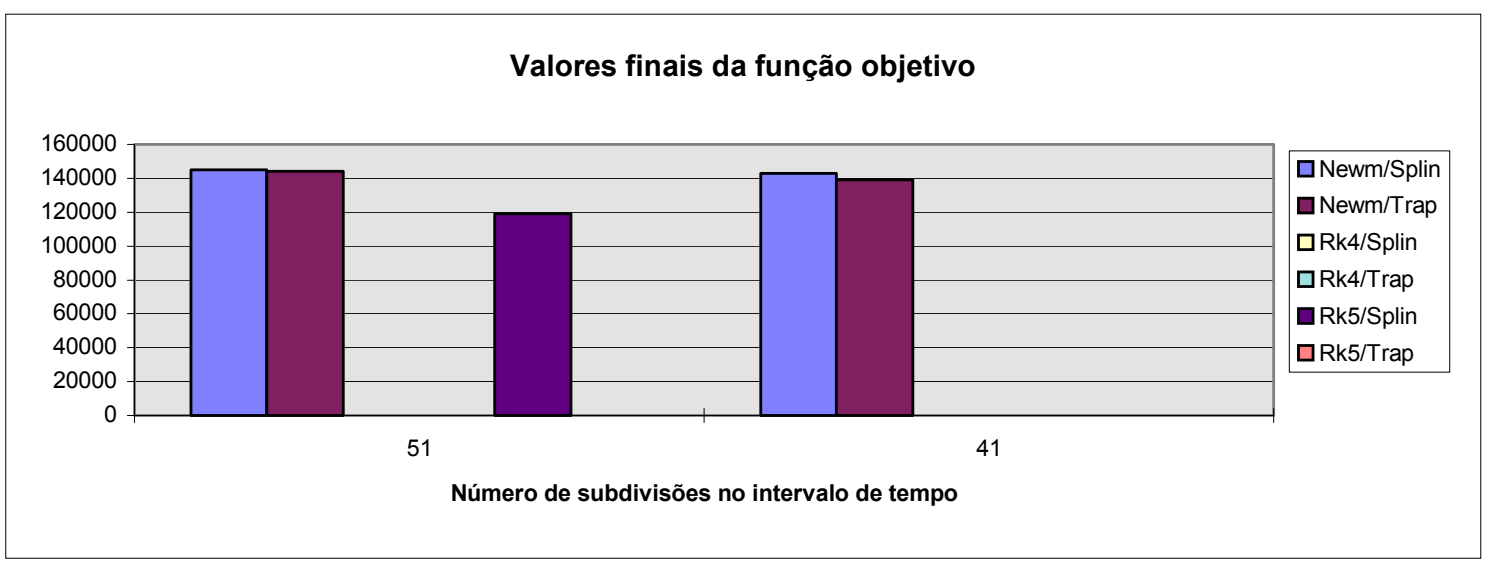

Gráfico 5.55 - Valores finais da função objetivo no caso 1 para diferentes valores de $n$.

No gráfico acima observa-se pouca sensibilidade das combinações com o método de Newmark em relação à variação do valor inicial de $\boldsymbol{x}$. 


\subsection{OBSERVAÇÕES FINAIS}

Analisando os exemplos acima pode-se observar os seguintes fatos:

1) O valor inicial de $\boldsymbol{x}$, nos exemplos 5.1 à 5.6, pouco interfere no valor de $\boldsymbol{x}_{f}$. Porém quando o valor inicial não está contido na região viável o número de minimizações sem restrições aumenta.

2) Nos exemplos com até cinco graus de liberdade todas as combinações de métodos apresentaram desempenhos equivalentes em relação aos parâmetros adotados. Já nos exemplos acima de cinco graus de liberdade as combinações com splines cúbicos obtiveram melhores performances, tanto em relação ao número de minimizações, quanto ao valor final da função objetivo.

3) Nos exemplos em que a equação do movimento é linear, as combinações com o algoritmo de Newmark se mostraram menos sensíveis, tanto em relação à variação de $n$, quanto de $\boldsymbol{x}_{0}$. Nos exemplos não-lineares as combinações com o método Runge-Kutta de quinta ordem apresentaram melhores desempenhos em relação aos parâmetros adotados.

4) $\mathrm{O}$ passo do vetor gradiente variando de $10^{-4}$ à $10^{-8}$ pouco interfere nos valores finais de $f(\boldsymbol{x})$ e de $k$ apresentados pelo método do Lagrangeano Aumentado.

5) O valor da variável $\rho$ no algoritmo de Armijo, página 57, variou nos exemplos acima resolvidos. Neste trabalho adotou-se o valor $\left\|\boldsymbol{x}_{\mathrm{o}}\right\|_{\infty_{\infty}} / 10$ que após alguns testes mostrou-se mais adequado.

6) O exemplo 5.8 (shear-building com 50 graus de liberdade) apresentou problemas. Destes, o mais marcante foi o da não convergência do algoritmo dos multiplicadores quando utilizadas as combinações com os métodos Runge-Kutta de quarta e quinta ordem. Nestas combinações, apenas o método Runge-Kutta de quinta ordem com splines cúbicos no caso 1 para $n=51$ apresentou convergência. Obteve-se convergência do algoritmo quando se utilizou combinações com o método de Newmark, independente dos algoritmos de integração numérica, de $n$ e de $\boldsymbol{x}_{\mathrm{o}}$.

As observações acima permitem concluir que problemas de pequeno porte utilizando o método do Lagrangeano aumentado são pouco sensíveis em relação às variações tanto dos métodos numéricos quanto aos parâmetros envolvidos. Entretanto, verifica-se que o método do Lagrangeano aumentado torna-se muito sensível aos algoritmos mencionados à medida que aumenta o número de graus de liberdade. 


\section{Capítulo 6}

\section{CONCLUSÃO}

O presente trabalho apresentou aplicações do Lagrangeano aumentado em otimização estrutural e mecânica com restrições dinâmicas.

Devido ao grande enfoque em otimização dinâmica, foi necessário dedicar parte deste texto ao estudo dos conceitos de programação matemática. Além disso diversos métodos numéricos se tornaram necessários. Os métodos utilizados foram os seguintes:

- Integração numérica:

1) Interpolação via splines cúbicos e a seguir integração com quadratura de Gauss-Legendre;

2) Regra do trapézio.

- Na resolução dos sistemas lineares, Cholesky.

- Integração da equação do movimento:

1) Método de Newmark quando a equação do movimento for linear;

2) Método Runge-Kutta de quarta ordem;

3) Método Runge-Kutta de quinta ordem. 
Estes métodos foram combinados e implementados em conjunto com o Lagrangeano aumentado. Diversos exemplos foram testados e a sensibilidade em relação aos métodos e alguns parâmetros foi analisada.

Nos exemplos de pequeno porte o método do Lagrangeano aumentado se comportou bem e houve pouca variação, tanto dos valores finais de $f(\boldsymbol{x})$ quanto de $k$, quando adotados diferentes métodos numéricos e parâmetros. Entretanto, conforme observado no item 5.9, o método torna-se muito sensível aos algoritmos de integração da equação do movimento, à medida que o número de graus de liberdade aumenta. Este fato requer um estudo mais aprofundado, uma vez que o método de Newmark aqui utilizado (única combinação à convergir incondicionalmente) resolve apenas sistemas de equações diferenciais ordinárias linear e portanto uma nova análise deverá ser feita para sistemas não lineares de grande porte.

A questão da avaliação de métodos de solução de sistemas não-lineares de grande porte e uma análise de sensibilidade mais profunda ficam como sugestões para futuros trabalhos a serem realizados. 


\section{REFERÊNCIAS BIBLIOGRÁFICAS}

[1] A. I. Chahande e J. S. Arora, "Optimization of large structures subjected to dynamic loads with the multiplier method", International Journal For Numerical Methods In Engineering, 37, 413 à 430, 1994.

[2] A. M. Calaes, Cálculo Matricial, Editora da UFOP, Ouro Preto, 1984.

[3] C. C. Hsieh e J. S. Arora, "Design sensitivity analysis and optimization of dynamic response", Computer Methods Applied In Mechanics And Engineering, 43, 195 à $219,1984$.

[4] C. H. Tseng e J. S. Arora, "Optimum design of systems for dynamics and controls using sequential quadratic programming”, AIAA Journal, 27, 1793 à 1800, 1989.

[5] D. G. Luemberger, Linear and Nonlinear Programming, 2a . edição, AddisonWesley Publishing Company, USA, 1984.

[6] D. S. Burnett, Finite Element Analysis, Addison Wesley, USA, 1987.

[7] E. J. Haug e J. S. Arora, Applied Optimal Design, Wiley-Interscience, New York, 1979.

[8] E. Polak, Computational Methods in Optimization - A Unified Approach, Academic Press, New York, 1971.

[9] G. H. Golub e C. F. Van Loan, Matrix Computations, $2^{\text {a }}$. edição, Hohns Hopkins University Press, 1989. 
[10] J. K. Paeng e J. S. Arora, "Dynamic response optimization of mechanical systems with multiplier methods", J. Mech. Tran. Auto. Design ASME, 111, 73 à 80, 1989.

[11] J. S. Arora, A. I. Chahande e J. K. Paeng, "Multiplier methods for engineering optimization", International Journal For Numerical Methods In Engineering, 32, 1485 à $1525,1991$.

[12] K. Atkinson, Elementary Numerical Analysis, Wiley, Singapore, 1993.

[13] L. W. Johnson e R. D. Riess, Numerical Analysis, Addison Wesley, USA, 1982.

[14] M. S. Medrano, Aplicações de Programação Matemática em Análise Estrutural, Dissertação Apresentada à Escola Politécnica da Universidade de São Paulo para obtenção do título de Mestre em Engenharia, São Paulo, 1994.

[15] N. Kikuchi, Finite Element Methods in Mechanics, Cambridge University Press, Cambridge, 1986.

[16] N. Piskunov, Cálculo Diferencial e Integral, Mir Moscú, URSS,1979.

[17] P. Goldenberg, M. A. Silva, P. M. Pimenta e R. M. L. R. F. Brasil, "Augmented Lagrangian Optimization of a Portal Frame with Elastoplastic Behavior under Dunamic Loading”. Fifth Pan American Congress on Applied Mechanics, Puerto Rico, Anais, 373 à 376, 1997.

[18] P. Goldenberg e P. M. Pimenta, Programação Matemática Aplicada à Engenharia de Estruturas, Notas de Aula, Escola Politécnica da Universidade de São Paulo, 1994.

[19] P. Goldenberg e P. M. Pimenta, Métodos Numéricos Aplicados à Mecânica das Estruturas I, Notas de Aula, Escola Politécnica da Universidade de São Paulo, 1994.

[20] P. M. Pimenta, Fundamentos da Teoria de Estruturas, Notas de Aula, 2 Volumes, Escola Politécnica da Universidade de São Paulo,1994.

[21] P. M. Pimenta, P. Goldenberg e R. M. L. R. F. Brasil, "Aplicações do Lagrangeano Aumentado em Otimização Estrutural”, Boletim Técnico EPUSP, BT/PEF-9513,1995.

[22] P. M. Pimenta, P. Goldenberg, R. M. L. R. F. Brasil e M. A. Silva, "Aplicações do Lagrangeano Aumentado em Otimização Estrutural com Restrições Dinâmicas”, IX Congresso de Métodos Numéricos y sus Aplicaciones, Bariloche, Anais, 1995. 
[23] P. M. Pimenta, P. Goldenberg, R. M. L. R. F. Brasil e M. A. Silva, "Aplicações do Lagrangeano Aumentado em Otimização Estrutural com Restrições Dinâmicas", Jornadas Internacionales de Mecânica Computacional y CAD/CAM, Concepcion, Chile, Anais, 185 à 192, 1995.

[24] P. M. Pimenta, P. Goldenberg, R. M. L. R. F. Brasil e M. A. Silva, "Otimização Estrutural com Restrições Dinâmicas pelo Método do Lagrangiano Aumentado", XVI Congresso Ibero Latino Americano sobre Métodos Computacionais para Engenharia, Curitiba, Anais, 1264 à 1272, 1995.

[25] R. Fletcher, Practical Methods of Optimization, Wiley, Great Britain, 1985.

[26] S. C. Chapra e Canale, Numerical Methods for Engineers, McGraw-Hill, USA, 1988. 


\section{ERRATA}

\begin{tabular}{|c|c|c|c|}
\hline Página & Linha & Onde se Lê & Leia-se \\
\hline 2 & 6 & são & é \\
28 & 25 & efetuados & efetuadas \\
30 & 20 & usado & utilizada \\
35 & 25 & $\theta=\mathbf{0}$ & $\theta_{0}=\mathbf{0}, r_{i}=1.0, i=1, m$, \\
41 & 13 & é & for \\
50 & 4 & uma função & um conjunto de funções \\
67 & 6 & $\sigma_{e} 20 M P a$ & $\sigma_{e}=20 M P a$ \\
129 & 1 & BILIOGRÁFICAS & BIBLIOGRÁFICAS \\
\hline
\end{tabular}

